University of Louisville

ThinkIR: The University of Louisville's Institutional Repository

Electronic Theses and Dissertations

$12-2013$

\title{
4D ultra-short TE (UTE) phase-contrast MRI for assessing stenotic flow and hemodynamics.
}

Mo Kadbi

University of Louisville

Follow this and additional works at: https://ir.library.louisville.edu/etd

\section{Recommended Citation}

Kadbi, Mo, "4D ultra-short TE (UTE) phase-contrast MRI for assessing stenotic flow and hemodynamics." (2013). Electronic Theses and Dissertations. Paper 719.

https://doi.org/10.18297/etd/719

This Doctoral Dissertation is brought to you for free and open access by ThinkIR: The University of Louisville's Institutional Repository. It has been accepted for inclusion in Electronic Theses and Dissertations by an authorized administrator of ThinkIR: The University of Louisville's Institutional Repository. This title appears here courtesy of the author, who has retained all other copyrights. For more information, please contact thinkir@louisville.edu. 


\title{
4D ULTRA-SHORT TE (UTE) PHASE-CONTRAST MRI FOR ASSESSING STENOTIC FLOW AND HEMODYNAMICS
}

\author{
By \\ Mo Kadbi \\ B.S., BE, University of Shahed, 2003 \\ M.S., BME, Sharif University of Technology, 2006 \\ A Dissertation \\ Submitted to the Faculty of the \\ J. B. Speed School of Engineering of the University of Louisville \\ in Partial Fulfillment of the Requirements \\ for the Degree of \\ Doctor of Philosophy \\ Department of Electrical and Computer Engineering \\ University of Louisville
}

December 2013 
Copyright 2013 by Mo Kadbi

All rights reserved 



\title{
4D ULTRA-SHORT TE (UTE) PHASE-CONTRAST MRI FOR ASSESSING STENOTIC FLOW AND HEMODYNAMICS
}

\author{
By \\ Mo Kadbi \\ B.S., BE, University of Shahed, 2003 \\ M.S., BME, Sharif University of Technology, 2006
}

A Dissertation Approved on

December 4, 2013

By the following Dissertation Committee

Dr. Amir A. Amini

Dr. Jacek M. Zurada

Dr. Marcus F. Stoddard

Dr. Guruprasad A. Giridharan

Dr. Tamer Inanc 


\section{DEDICATION}

This dissertation is dedicated to the memory of my father, who emphasized the importance of education and helped me with my lessons throughout his life.

This dissertation is lovingly dedicated to my mother who is dealing with me being a world away. Thank you for your support, encouragement, and constant love which has sustained me throughout my life.

Finally, this dissertation is dedicated to my sisters who have been a great source of motivation, inspiration, and endless love. 


\section{ACKNOWLEDGEMENTS}

This dissertation project would not have been possible without the support of many people. Firstly, I would like to express my sincere gratitude to my supervisor Dr. Amir Amini who has encouraged and offered me invaluable assistance, support, and guidance through this work. Deepest gratitude is also due to the members of my dissertation committee, Dr. Zurada, Dr. Inanc, Dr. Giridharan, and Dr. Stoddard. Special thanks also to all my graduate friends and classmates who provided moral support. MJ Negahdar and I have been working in parallel on our PhD studies since the start. I would like to thank MJ for all the good discussions, input, and endless help. Special thanks to my friend Dr. Hui Wang, formerly with the Medical Imaging Lab and now with Philips Medical Systems who always has been willing to discuss different matters regarding my project.

I am grateful to have had the opportunity to work with MR Clinical science group at Philips healthcare North America, and would like to thank Dr. Stephen Fischer (1963-2011), Dr. Peter Martin, and Dr. Melanie Traughber who generously shared their expertise and contributed with their knowledge of MRI.

Finally, I wishes to express his love and gratitude to his beloved family; for their understanding and endless love, through the duration of my studies. 


\title{
ABSTRACT
}

\section{ULTRA-SHORT TE (UTE) PHASE-CONTRAST MRI FOR ASSESSING STENOTIC FLOW AND HEMODYNAMICS}

\author{
Mo Kadbi
}

December 4, 2013

Phase-contrast (PC) MRI is a non-invasive technique to assess cardiovascular blood flow. However, this technique is not accurate in the case of atherosclerotic disease and vascular and valvular stenosis due to intravoxel dephasing secondary to disturbed blood flow, flow recirculation, and turbulence distal to the narrowing, resulting in flow-related artifacts. Previous studies have shown that reducing the echo time (TE) decreases the errors associated with phase incoherence due to random motions as observed in unsteady and turbulent flows. As part of this dissertation, a novel 3-D cine Ultra-Short (UTE)-PC imaging method has been developed, and implemented to measure the blood velocity using a UTE center-out radial k-space trajectory with short TE time compared to standard PC MRI sequences. 3D UTE characterizes flow in one direction in a 3D volume, resulting in a single component of the flow velocities. In order to obtain a comprehensive flow assessment in three directions, the 3D UTE sequence needs to be repeated three times, which can be inefficient and time consuming. 4-D flow MRI has been recently used for quantitative flow 
assessment and visualization of complex flow patterns resulting in more anatomical information and comprehensive assessment of blood flow. With 4D flow MRI method, all the flow information in three direction in a $3 \mathrm{D}$ volume though the time can be achieved as part of a single scan. In this dissertation, a novel 4D UTE flow MRI technique has also been designed and implemented which is capable of deriving the three orthogonal components of the velocity field in the flow in a single scan, while achieving very short echo times. In flow phantom studies, comprehensive investigation of several different flow rates revealed significant improvement in flow quantification and reduction of flow artifacts when compared to conventional 4D flow. Furthermore, a reduced TE 4D Spiral flow MRI method has also been implemented which reduces scan times when compared to conventional 4D flow MRI (as well as 4D UTE flow). Despite reduction of scan time as well as TE relative to conventional 4D flow, the achieved TE with the 4D spiral technique is indeed longer than 4D UTE flow.

In order to assess clinical feasibility and in order to perform further validation of 4D UTE flow, in an IRB-approved study, twelve aortic stenosis (AS) patients underwent Doppler Ultrasound, conventional 4D flow, and 4D UTE flow scans for a 3 way comparison. 4D UTE flow displayed good correlation with Doppler Ultrasound in patients with moderately severe aortic stenosis, though with the added benefit of not having confounding factors encountered in Doppler Ultrasound (e.g., angle dependence, 2D measurement, and difficulty in locating a proper acoustic window). The proposed 4D UTE flow permits 4D visualization of flow and true 3D measurement of all flow quantities, not possible with Doppler. Further investigations will be required to test the technique in patients with severe or critical aortic stenosis wherein conventional 4D flow will be less accurate due to intravoxel dephasing and spin incoherence. 


\section{TABLE OF CONTENTS}

DEDICATION ………………………………………………………………………………… III

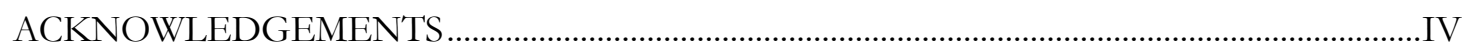

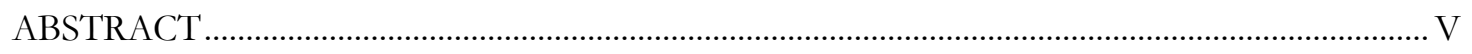

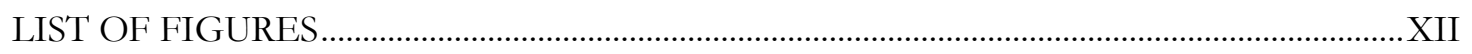

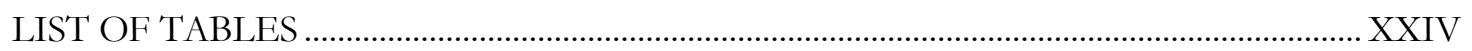

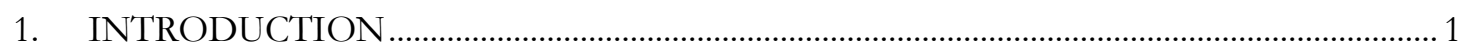

2. PHYSIOLOGY OF BLOOD FLOW, ANATOMY OF THE VESSEL WALL, AND

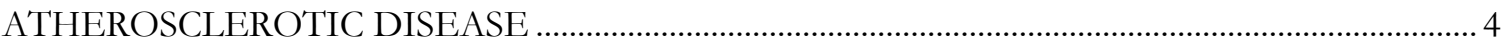

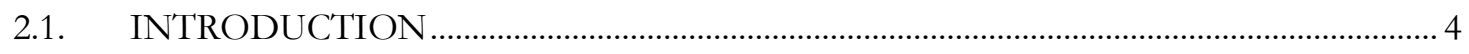

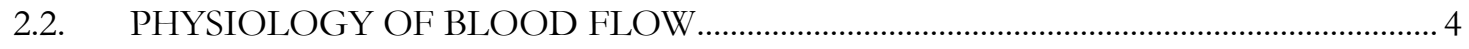

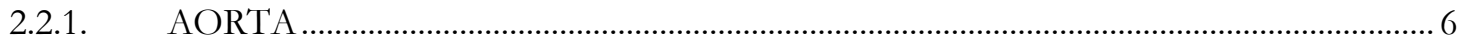

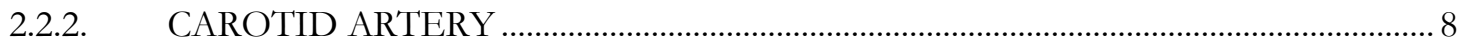

2.3. ANATOMY OF THE VESSEL WALL AND ATHEROSCLEROTIC DISEASE ......... 9

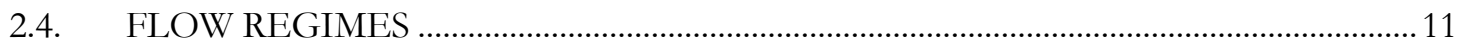

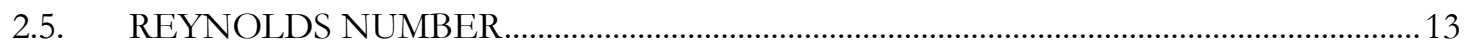

2.6. DISTURBED AND TURBULENT BLOOD FLOW …………….................................... 14

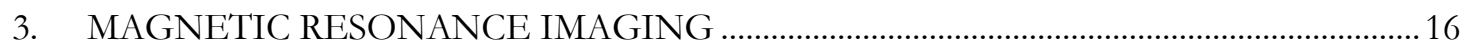


3.1. PROTON MAGNETIC RESONANCE AND NET MAGNETIZATION

3.2. RESONANCE PHENOMENA AND SIGNAL GENERATION …...............................17

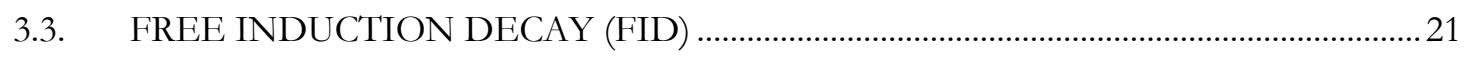

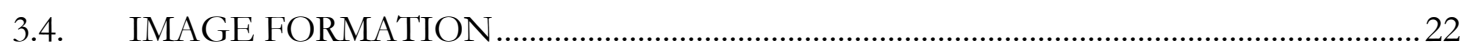

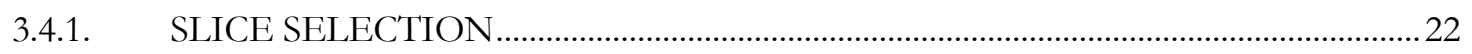

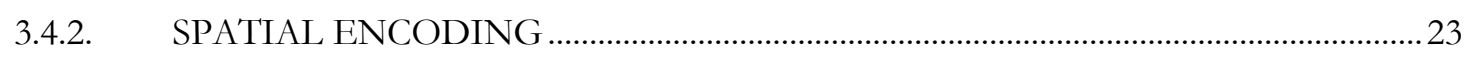

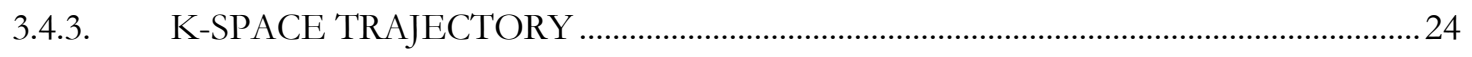

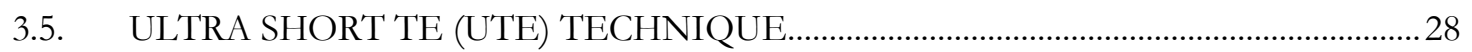

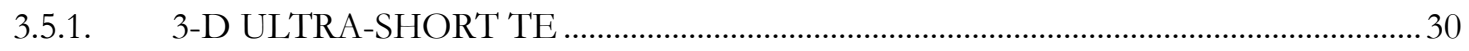

3.5.2. KOOSHBALL AND STACK-OF-STAR TRAJECTORIES .......................................... 32

4. FLOW IMAGING AND MAGNETIC RESONANCE ANGIOGRAPHY (MRA) ...........34

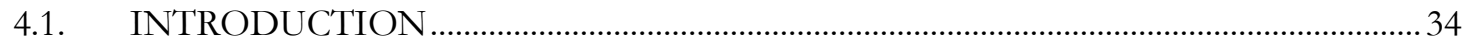

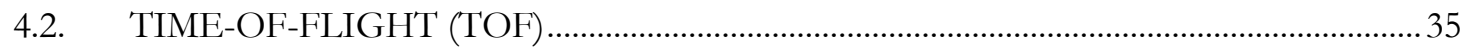

4.3. PHASE CONTRAST ANGIOGRAPHY (PCA) ................................................................. 36

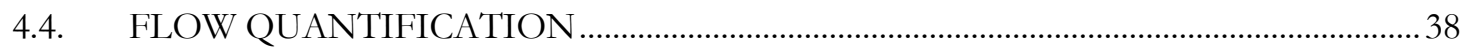

5. DISTURBED AND TURBULENT FLOW QUANTIFICATION ........................................ 41

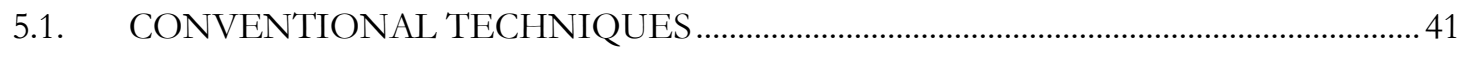

5.2. ULTRA SHORT TE (UTE) TECHNIQUE......................................................................... 43

5.3. SHORTCOMINGS OF PREVIOUS UTE-PC MRI TECHNIQUE ................................... 43

5.4. 3-D UTE PHASE-CONTRAST (PC) MRI....................................................................... 45

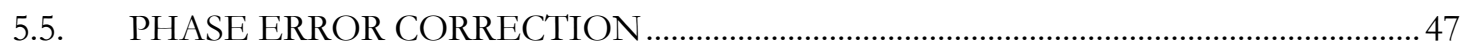


5.6. PHASE ERROR CORRECTION AND CALIBRATION USING STATIC

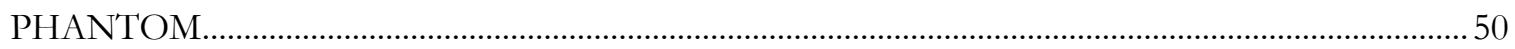

5.7. STEADY FLOW IN STENOTIC FLOW PHANTOM....................................................... 51

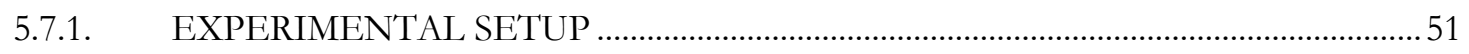

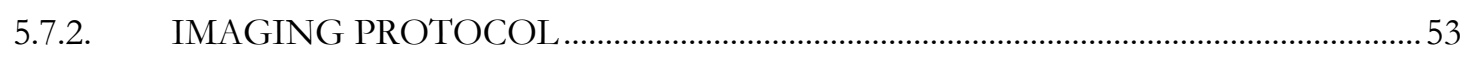

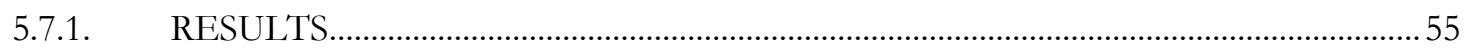

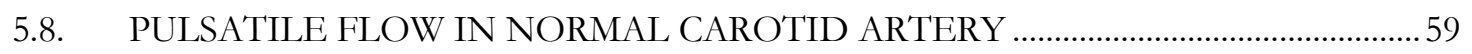

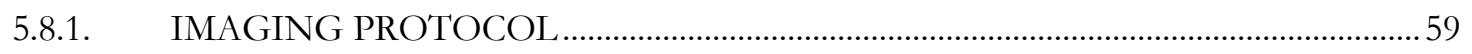

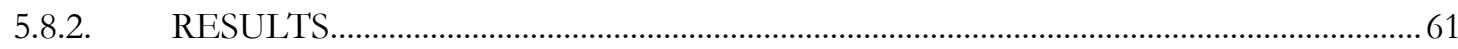

6. 3-D UTE PC MRI: THE EFFECT OF VENC, SAMPLING DENSITY, GRADIENT

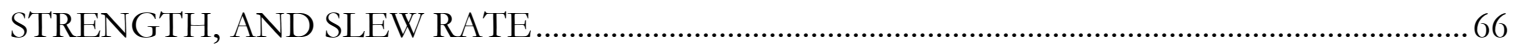

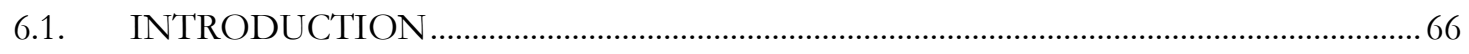

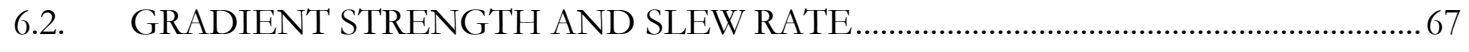

6.3. CENTER-OUT RADIAL SPOKE NUMBER VARIATION ............................................ 68

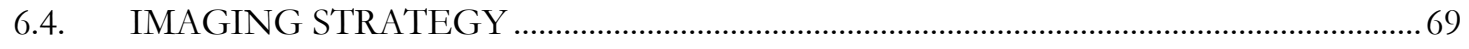

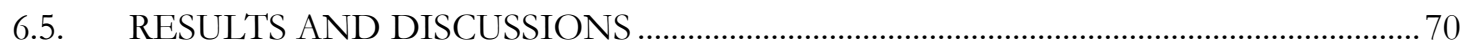

7. REDUCED TE 4D FLOW SPIRAL MRI FOR ASSESSMENT OF FLOW AND

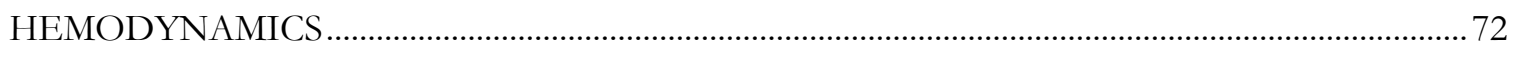

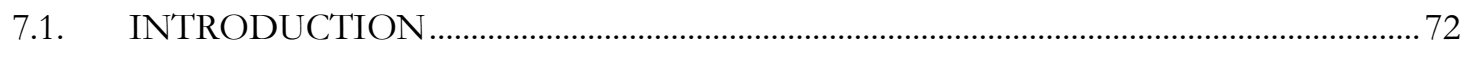

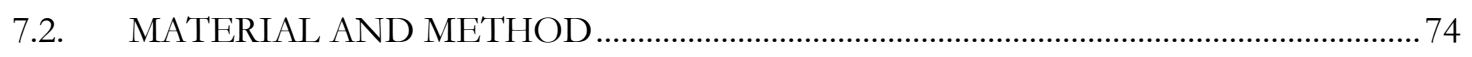

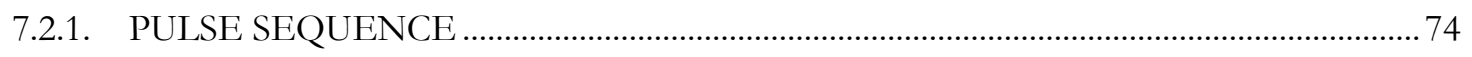

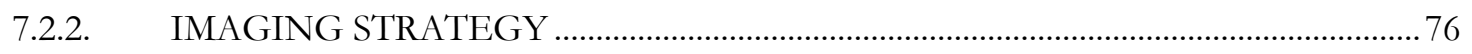


7.3. RESULTS AND DISCUSSIONS .....

8. 4D UTE FLOW: A PHASE-CONTRAST MRI TECHNIQUE FOR ASSESSMENT OF

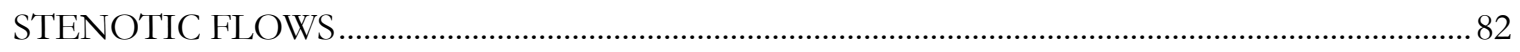

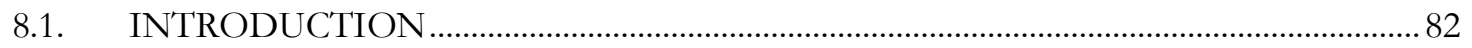

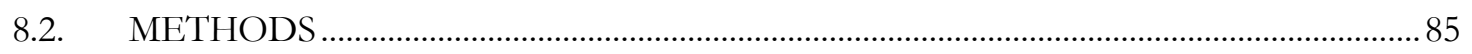

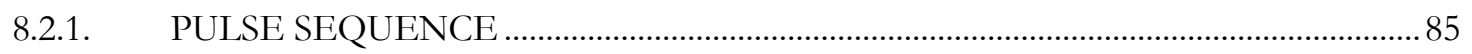

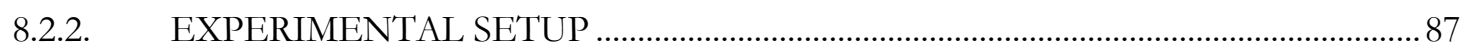

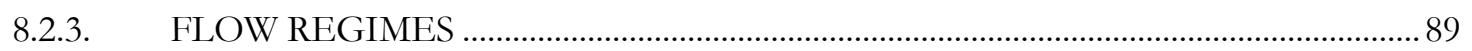

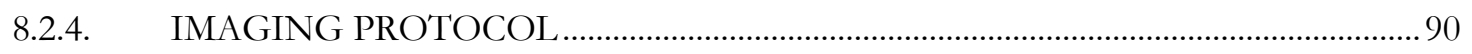

8.2.5. PHASE CORRECTIONS AND ROI DEFINITIONS ................................................. 91

8.3. RESULTS

8.3.1. NUMBER OF PROJECTIONS AND ITS EFFECT ON SCAN TIME AND ACCURACY 93

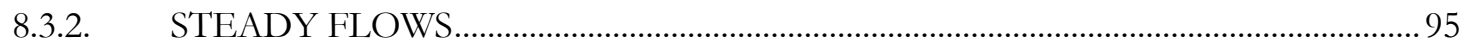

8.3.3. PULSATILE FLOWS................................................................................................ 101

8.3.1. VALIDATION EXPERIMENTS WITH THE LB PUMP...........................................107

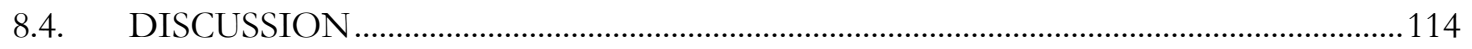

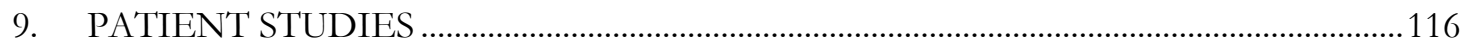

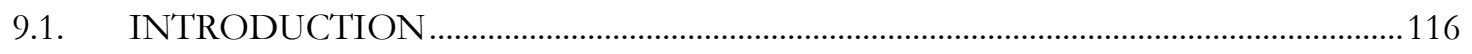

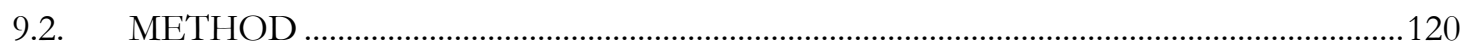

9.2.1. PATIENT POPULATION AND CASES .................................................................... 120

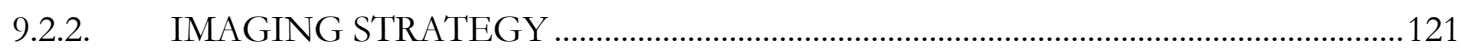


9.2.3. DATA ANALYSIS

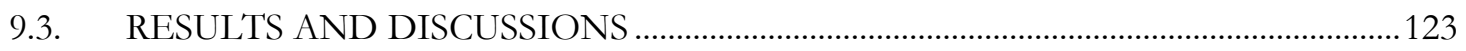

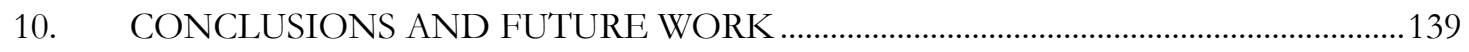

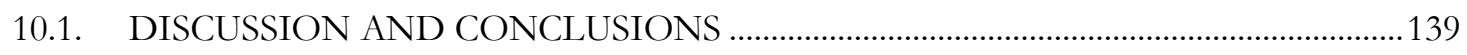

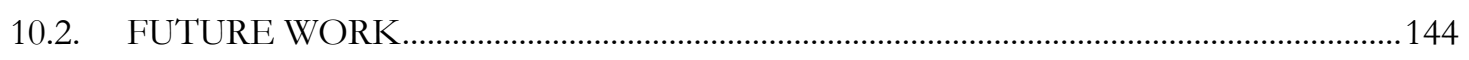

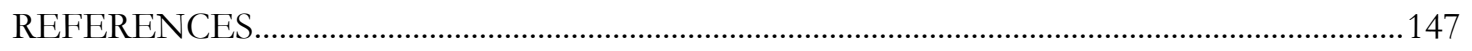

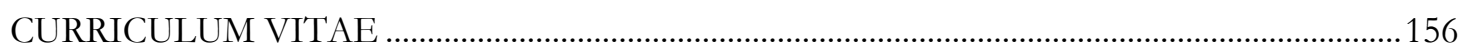




\section{LIST OF FIGURES}

Figure 1: A schematic of blood velocity and pressure in the vascular tree. The blood velocity decreases as one move away from the heart while the pressure increases. Taken

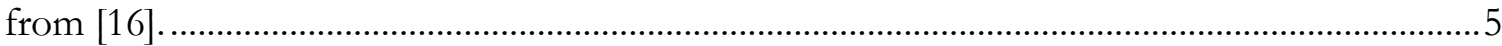

Figure 2: A schematic of aorta and its main branches (taken from [17])(a), and arterial pressure curve including systole, dicrotic notch and, diastole (taken from [18])(b)..................6

Figure 3: Carotid artery and its branches in left and right side of neck (taken from [22])...8

Figure 4: Visualization of flow in a glass model of carotid bifurcation[15]. ......................... 9

Figure 5: The three layers of vessels walls (taken from [24]).............................................10

Figure 6: Narrowing in a blood vessel due to accumulation of plaque (taken from [28]). 11

Figure 7: A schematic of laminar flow (left column) and turbulent flow distal to stenosis (right column). The bottom row shows the velocity profile for these flows [30].....

Figure 8: Spinning nuclei induces a magnetic moment which behaves like a bar magnet [58] .16

Figure 9: Precession of net magnetization $M$ around the $B_{0}$ field by applying the $B_{1} R F$ pulse (taken from [58]). 18

Figure 10: longitudinal and transverse magnetization during relaxation. T1 and T2 are independent and T2 is always shorter than T1 [62].

Figure 11: $T_{2}$ relaxation process. As spin start to precess, the magnitude of its $M_{x y}$ component is reduced [64] 
Figure 12: Exponential decay in detected signal. Due to $\mathrm{B}_{0}$ inhomogeneity the decreasing of signal is accelerated such that $\mathrm{T} 2 *$ is significantly shorter than $\mathrm{T}_{2}[58] \ldots$

Figure 13: A typical free induction decay signal and measurement of a time based signal using RF receive coil [64]......

Figure 14: A typical k-space domain. $\mathrm{K}_{\mathrm{x}}$ denotes the direction of frequency encoding and Ky denotes the direction of phase encoding [66]....... .24

Figure 15: A schematic of gradient echo pulse sequence (a) which sample k-space lines in Cartesian fashion (b) [65]

Figure 16: a schematic of spiral sequence with radial readout in two direction (a), and one interleave of spiral trajectory in k-space domain (b) [65]. .26

Figure 17: Radial pulse sequence with radial readout out in two directions (a), and radial trajectories in k-space domain showing oversampling of center of k-space in this method (b) $[65]$ .27

Figure 18: Conventional 2-D UTE sequence based on two back to back scan with half RF pulse [75]. .28

Figure 19: The radial readout trajectory in 3-D UTE. $\mathrm{K}_{\max }$ is the radius of radial k-space which is determined by spatial resolution in three spatial directions [77]. .30

Figure 20: 3-D UTE sequence based on slice selective RF pulse using radial readout gradient in two directions. .31

Figure 21: 3-D UTE sequence based on non-selective RF pulse using radial readout gradient in two directions. TE can be reduced to 8 microseconds using this sequence [82]..32

Figure 22: Two 3-D trajectory acquisition. One interleave of kooshball trajectory (a) and 13 slices of stack-of-stars (b) is shown. The dot points are the end-point of projections in kspace. .33 
Figure 23: A schematic of blood refreshment TOF technique. Blood refreshment depends on flow velocity, volume thickness and repetition time TR .36

Figure 24: An example of flow sensitive gradient consisting of two lobes with identical shape. .37

Figure 25: Magnitude and phase image for flow sensitive and compensated scan using phase contrast angiography and measured phase velocity map by subtraction of the phase of the two scans[2]. .38

Figure 26: The proposed 3-D UTE-PC MRI sequence. The flow sensitive (encoding) and compensated scans are acquired through combination of a bipolar gradient and refocusing part of slice select gradient. Gradient $G_{z}$ is applied to acquire multiple slices in a volume using stack of star strategy. .45

Figure 27: Phantom result for different flow directions. The first row shows the original images using 3-D UTE-PC imaging in FH, AP, and RL flow direction respectively. The second row shows the result after phase error correction. .50

Figure 28: Schematic of the experimental setup showing the test Section with $87 \%$ area stenosis which was machined in-house from clear acrylic plastic. .52

Figure 29: A schematic of the geometry of the phantom with six ROI where data were collected. The dash line shows the position of a slice $33 \mathrm{~mm}$ distal to stenosis.

Figure 30: Magnitude image (first row), velocity image (second row), and velocity profile (third row) for low flow rate $\mathrm{Q}=13.2 \mathrm{~mL} / \mathrm{s}$ at $33 \mathrm{~mm}$ distal to throat of the stenosis using Cartesian (first column), spiral (second column), and 3-D UTE (third column) PC MRI sequences. The magnitudes, flows, and velocity profiles are in agreement for three techniques .54 
Figure 31: Magnitude image (first row), velocity image (second row), and velocity profile (third row) for medium flow rate $\mathrm{Q}=39.6 \mathrm{~mL} / \mathrm{s}$ at $33 \mathrm{~mm}$ distal to throat of the stenosis using Cartesian ( first column), spiral (second column), and 3-D UTE (third column) PC MRI sequences. .55

Figure 32: Measured flow rate (left column) and peak velocity ( right column) for Cartesian, spiral and 3-D UTE PC MRI sequences for low flow rate $Q=13.2 \mathrm{~mL} / \mathrm{s}$ along the length of the phantom (first row), medium flow rate $\mathrm{Q}=39.4 \mathrm{~mL} / \mathrm{s}$ (second row), and high flow rate $Q=300 \mathrm{~mL} / \mathrm{s}$ (third row). $\mathrm{Z}=0$ denotes throat of the stenosis and $\mathrm{Z}>0$ correspond to positions downstream of the stenosis as shown in Figure 29............................56

Figure 33: Magnitude image (first row), velocity image (second row), and velocity profile (third row) for high flow rate $\mathrm{Q}=300 \mathrm{~mL} / \mathrm{s}$ at $33 \mathrm{~mm}$ distal to throat of the stenosis for the case of Cartesian (first column), spiral (second column), and 3-D UTE (third column) acquisitions. The magnitudes images for Cartesian and spiral sequences show signal loss. The velocity image in the Cartesian acquisition reveals a significant phase error at the center of jet compared to spiral and 3-D UTE. There is negligible signal loss and phase error in 3-D UTE PC MRI. .58

Figure 34: Right CCA magnitude and phase image (left column) and peak velocity, mean velocity and flow waveform (right column) for conventional sequence (first row) 3-D UTEPC MRI without phase error correction (second row) and 3-D UTE-PC MRI with phase error correction (third row). Image slice from the M2D acquisition in (first row) and 3-D acquisition in (second row) and (third row) was located $5 \mathrm{~mm}$ proximal to bifurcation. .......60

Figure 35: The average blood flow in right (a) and left (b) CCA using multi-2D PC MRI (red line) and 3-D UTE-PC MRI after phase error correction (blue line). 
Figure 36: The average blood flow in right (a) and left (b) ECA using multi-2D PC MRI (red line) and 3-D UTE-PC MRI after phase error correction (blue line).....

Figure 37: The average blood flow in right (a) and left (b) ICA using multi-2D PC MRI (red line) and 3-D UTE-PC MRI after phase error correction (blue line)

Figure 38: A schematic of bipolar gradients in PC MRI. Blue shaded gradient has 10 $\mathrm{mT} / \mathrm{sec}$ strength and yellow shaded gradient benefits from higher gradient $20 \mathrm{mT} / \mathrm{sec}$ and shorter length.

Figure 39: Flow waveform in right CCA (top row) and ICA (bottom row) using conventional 3D PC MRI and 3D UTE PC MRI with various sampling, gradient strength, slew rate, and Venc.

Figure 40: A schematic of reduced TE 4D spiral PC MRI sequence with for consecutive scans including 3 flow sensitive scans in three flow directions and one reference scan. Subtraction of each phase image of flow sensitive scan from phase image of the reference scan results in PC velocities for the corresponding flow direction. Gradient $G_{z}$ is applied to acquire multiple slices in a volume using stack of star strategy..............................................75

Figure 41: Demonstration of (left) conventional single shot spiral acquisition with 10 rotations and (right) interleaved spiral acquisition with 10 interleaves. .75

Figure 42: Flow waveform for Right CCA (a) averaged in 12 slices proximal to bifurcation and for Right ICA (b) in 20 slices distal to bifurcation using Cartesian ( red cruve) and spiral (blue curve) PC MRI in four volunteers. .78

Figure 43: Bland-Altman plot in each cardiac phase in four normal volunteers demonstrating the mean flow difference between 4D conventional and 4D spiral flowin (a)RCCA and (b) LCCA. The blue dots are all measured flow values from 4 volunteers in all cardiac frames. Having 4 volunteers, 3 CCA slices in each volunteer, and 12 cardiac frames 
in each CCA results in $4 * 3 * 12=144$ data points (a). For ICA 5 slices are available distal to bifurcation in each volunteer and the number of data points is $4 * 5 * 12=240$ (b). The abscissa is the mean flow values from 4D conventional flow calculated over 12 slices during the cardiac cycle .79

Figure 44: Velocity profile for 10 slices along right carotid artery using 4D Cartesian flow (a) and 4D spiral flow (b) in a normal volunteer during systolic phase of the cardiac cycle...80

Figure 45: Flow pathlines systolic cardiac phase in right carotid artery acquired using (a) conventional 4D Cartesian PC MRI and (b) 4D spiral PC MRI.

Figure 46: Timing diagram of 4D flow MRI based on 4-point balanced scheme (a). Schematic for conventional 4D flow MRI (b) and 4D UTE flow MRI sequence (c). In both techniques, four consecutive k-space lines per cardiac frame including three flow sensitive in three flow directions and one reference frame are acquired. Gray shaded gradients indicate the flow sensitive gradients in each flow direction. Subtracting the phase of reference image from each of flow sensitive scans results in flow measurement in three directions. .86

Figure 47: A schematic geometry of the phantom and the sagittal extent of the FOV for data collection. Dashed lines represent the location of axial slices along the phantom. The green, red, and blue areas are regions proximal, at the throat, and distal to the stenosis that

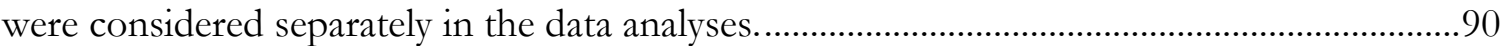

Figure 48: Linear regression analysis at three locations proximal (first row), at the throat (second row), and distal (third row) to stenosis using conventional 4D flow MRI (a) and 4D UTE flow MRI (b). The x-axis is the prescribed flow at the pump and y-axis the measured flow using two sequences. The Shelley pump was used............................................................. 96

Figure 49: Measured flow at each slice along the phantom at $Q=150 \mathrm{ml} / \mathrm{s}$ steady flow. The green plot shows the measured flow for the flow-off experiment using UTE sequence 
and the flow value diverges away from zero towards the end of the coil. The errors for UTEderived flow rate (blue plot) was reduced using flow-off experiment as shown by the black

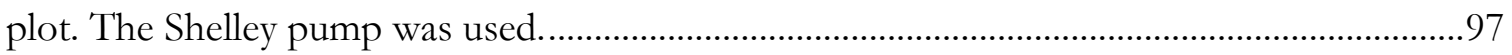

Figure 50: An axial slice $21 \mathrm{~mm}$ distal to throat of the stenosis at $\mathrm{Q}=50 \mathrm{ml} / \mathrm{s}$ steady flow rate using conventional 4D flow (top row) and 4D UTE flow (bottom row). The magnitude (a), phase (b) and velocity contour (c) indicate a good agreement between two sequences at low flow rates. The Shelley pump was used................................................................................ 98

Figure 51: A sagittal view of phantom at the throat of stenosis at $Q=50 \mathrm{ml} / \mathrm{s}$ steady flow rate. The velocity profile (first row) and flow streamlines (second row) are shown using conventional 4D flow MRI (a) and 4F UTE flow MRI (b). Velocity profiles proximal, at the throat, and distal to stenosis show an excellent agreement between two sequences. Flow streamlines at three locations display good correlation in both sequences. The Shelley pump was used. .99

Figure 52: An axial slice $21 \mathrm{~mm}$ distal to the throat of the stenosis for the case of steady flow at $\mathrm{Q}=300 \mathrm{ml} / \mathrm{s}$. The magnitude (a), phase (b), and velocity contours (c) from conventional 4D flow MRI (top row) as well as 4D UTE flow MRI (bottom row) are displayed. The intravoxel dephasing and signal loss is visible in the case of long TE (top row). The removal of intravoxel dephasing and signal loss in the UTE sequence is appreciable. The Shelley pump was used.... 100

Figure 53: A sagittal view of phantom at $\mathrm{Q}=300 \mathrm{ml} / \mathrm{s}$ steady flow rate. The velocity profile (first row) and flow streamlines (second row) are shown for conventional 4D flow MRI (a) and 4D UTE flow MRI (b). The flow-related artifact and intravoxel dephasing (yellow arrows) caused inaccurate velocity profile and streamlines in conventional sequence. The Shelley pump was used. 100 
Figure 54: Linear regression analysis for measured pulsatile flow rates between conventional 4D flow MRI and 4D UTE flow MRI at three locations: proximal (a), throat (b), and distal (c) to the stenosis. Each data point represents the measured flow rate for a specific cardiac phase averaged in 3, 2, and 12 slices proximal, throat, and distal to stenosis. The data points show more difference in flow measurement at high flow rates. The measurement at the throat of the stenosis has the highest correlation between two techniques at all flow rates. The Shelley pump was used.

Figure 55: Prescribed flow waveform (a). The peak systolic flow rate (as well as the entire waveform) is adjusted to reflect Qmax $=50 \mathrm{ml} / \mathrm{s}$ (first column), Qmax=150 ml/s (second column), and $\mathrm{Qmax}=300 \mathrm{ml} / \mathrm{s}$ (third column). Measured flow waveform in an axial slice 12 $\mathrm{mm}$ proximal to the stenosis (b) and $21 \mathrm{~mm}$ distal to the stenosis (c) In the Figure, conventional 4D flow MRI measurements have been plotted in red and 4D UTE flowderived measurements have been plotted in blue. The flow waveforms reveal reasonable correspondence at 50 and $150 \mathrm{ml} / \mathrm{s}$; however the conventional sequence result in an erroneous flow waveform and a significant underestimation of peak flow distal to narrowing due to turbulence and intravoxel dephasing. The Shelley pump was used............................. 103

Figure 56: Measured peak systolic flow rate in slices along the length of the phantom for Qmax $=50,150$, and $300 \mathrm{ml} / \mathrm{s}$ using conventional 4D flow (red) and 4D UTE flow (blue). There is good agreement between the two sequences with Qmax=50 and Qmax $=150 \mathrm{ml} / \mathrm{s}$; however conventional 4D flow leads to a considerable variation in measured flow and a discernible underestimation distal to the narrowing. The Shelley pump was used.

Figure 57: Flow pathlines in sagittal view at the pulsatile flow rates $50 \mathrm{ml} / \mathrm{s}$ (top row) and $150 \mathrm{ml} / \mathrm{s}$ at peak systolic time(bottom row) using conventional 4D flow MRI (a) and 4D UTE flow MRI (b). The flow pathlines are in good agreement at $50 \mathrm{ml} / \mathrm{s}$ flow rates. A slight 
difference is visible between flow pathlines at $150 \mathrm{ml} / \mathrm{s}$ due to moderately high flow rates and turbulence distal to the center of stenosis. The Shelley pump was used.

Figure 58: Flow pathlines at peak systolic time for the pulsatile flow with $\mathrm{Qmax}=300$ $\mathrm{ml} / \mathrm{s}$ at peak systolic time using conventional 4D flow MRI (a) and 4D UTE flow MRI (b). The top row shows a sagittal view of the phantom including regions proximal and distal to the stenosis. The bottom row shows an enlarged view of distal regions. The error and inaccuracy in flow pathlines in the conventional sequence is evident. The Shelley pump was used. 106

Figure 59: Mean flow (over the entire cardiac cycle) at each slice along the phantom at $\mathrm{Q}=200 \mathrm{ml} / \mathrm{s}$ using conventional 4D flow MRI and 4D UTE flow MRI with flow-on and flow-off experiments and the result after phase error correction. The LB pump was used.109

Figure 60: Mean flow (over the entire cardiac cycle) at each slice along the phantom at $\mathrm{Q}=300 \mathrm{ml} / \mathrm{s}$ using conventional 4D flow MRI and 4D UTE flow MRI with flow-on and flow-off experiments and the result after phase error correction. The LB pump was used.110

Figure 61: Flow waveform measured using conventional 4D flow MRI and 4D UTE flow MRI at three locations proximal (a), at the stenosis (b) and distal to stenosis (c). The LB pump was used. 111

Figure 62: Flow waveform measured using conventional 4D flow MRI and 4D UTE flow MRI at three locations proximal (a), at the stenosis (b) and distal to stenosis (c). The LB pump was used. 111

Figure 63: A sagittal view of phantom at $Q=200 \mathrm{ml} / \mathrm{s}$ pulsatile flow rate at peak systolic time. The velocity profile and velocity contour at axial cross sections proximal, at the throat, and distal to narrowing are shown using conventional 4D flow MRI (first row) and 4D UTE flow MRI. Velocity profiles and contours reveal a good correlation between the two 
sequences. The LB pump was used. The darker phantom in the bottom figure is due to windowing level in the visualization software.

Figure 64: A sagittal view of phantom at $Q=300 \mathrm{ml} / \mathrm{s}$ pulsatile flow rate at peak systolic time. The velocity profile and velocity contour at axial cross sections proximal, at the throat, and distal to narrowing are shown using conventional 4D flow MRI (first row) and 4D UTE flow MRI (second row). Velocity profiles and contours reveal a good correlation between two sequences. The difference between two sequence distal to stenosis is slightly more than $\mathrm{Q}=200 \mathrm{ml} / \mathrm{s}$ due to higher flow rate. The LB pump was used.

Figure 65: A sagittal view of phantom at $Q=400 \mathrm{ml} / \mathrm{s}$ pulsatile flow rate at peak systolic time. The velocity profile and velocity contour at axial cross Sections proximal, at the throat, and distal to narrowing are shown using conventional 4D flow MRI (first row) and 4D UTE flow MRI (second row). Velocity profiles and contours reveal a good correlation between two sequences. The difference between two sequence distal to stenosis is more than $Q=200$ and $300 \mathrm{ml} / \mathrm{s}$. The LB pump was used. 114

Figure 66: Bland-Altman plot at aortic valve in eightpatients demonstrating the peak velocity difference between 4D conventional (a) and 4D UTE flow (b) with Doppler echo. 126

Figure 67: Flow versus time waveforms at the level of the aortic valve (a), and $15 \mathrm{~mm}$ distal to the aortic valve (b) in subject 4 with AS (first row), subject 5 with AR (second row), and subject 6 with AS and AR (third row) using conventional 4D flow MRI (red plot) and 4D UTE flow MRI(blue plot).

Figure 68: Flow versus time waveform at the level of the aortic valve (a), and $15 \mathrm{~mm}$ distal to the aortic valve (b) in subject 7 with AS and AR (first row), subject 8 with AS ( 
second row), and subject 9 with AS and AR (third row) using conventional 4D flow MRI (red plot) and 4D UTE flow MRI(blue plot).

Figure 69: Flow versus time waveforms at the level of the aortic valve (a), and $15 \mathrm{~mm}$ distal to the aortic valve (b) in subject 10 with AS and AR (first row), subject 11 with AS (second row), and subject 12 with AS and AR (third row) using conventional 4D flow MRI (red plot) and 4D UTE flow MRI (blue plot).

Figure 70: Bland-Altman analysis of LVOT diameter calculated from Doppler echo and 4D flow UTE MRI (a), Doppler echo and conventional 4D flow MRI (b), and conventional 4D flow MRI and 4D flow UTE MRI (c).

Figure 71: Magnitude and phase of conventional 4D flow MRI (a) and 4D UTE flow MRI (b) in subject 8 with porcine valve replacement at the level of the aortic valve. Susceptibility artifacts can be seen in (a)

Figure 72: Velocity profile in 3D and 2D view using conventional 4D flow MRI (a) and 4D UTE flow MRI (b) in subject 8 who had porcine valve replacement at the aortic valve. Susceptibility artifacts in both views of the conventional technique is clear.

Figure 73: Velocity magnitude and profile at the level of aortic valve in subject 4 with AS at peak systolic time using conventional 4D flow (a) and 4D UTE flow (b).....

Figure 74: Velocity magnitude and profile in subject 5 with AR at peak systolic time (first row) and during AR (second row) using conventional 4D flow (a) and 4D UTE flow (b). 133

Figure 75: Velocity magnitude and profile in subject 6 with AS and AR at peak systolic time (first row) and during AR (second row) using conventional 4D flow and 4D UTE flow (b). 134 
Figure 76: Velocity profile at aortic valve in subject 7 with AS and AI using conventional 4D flow MRI (a) and 4D UTE flow MRI (b). The velocity profiles reveal a good correspondence between two techniques. 136

Figure 77: Velocity magnitude and profile in subject 9 with AS and AR at peak systolic time using conventional 4D flow (a) and 4D UTE flow (b). Velocity profile using 4D UTE flow MRI is different due to radial undersampling and streaking artifact which yields incorrect velocities (arrows). 136

Figure 78: Velocity magnitude and profile in subject 10 with AS and AR at peak systolic time using conventional 4D flow (a) and 4D UTE flow (b). There is a good correlation between velocity magnitude and profile measured by two techniques.

Figure 79: Velocity magnitude and profile in subject 11 with AS at peak systolic time using conventional 4D flow (a) and 4D UTE flow (b). Velocity profile using conventional 4D flow MRI shows more flow artifacts due to high velocities present. 137

Figure 80: Velocity magnitude and profile in subject 12 with AS at peak systolic time using conventional 4D flow (a) and 4D UTE flow (b). Arrows show the similarity of two techniques in determining the shape of valve narrowing from velocity magnitude. 138 


\section{LIST OF TABLES}

Table 1: Acquisition parameters at $\mathrm{Q}=13.2,39.4$, and $300 \mathrm{~mL} / \mathrm{s}$ for Cartesian, spiral, and 3-D UTE PC MRI for Phantom Studies. The Cartesian and the spiral acquisitions were

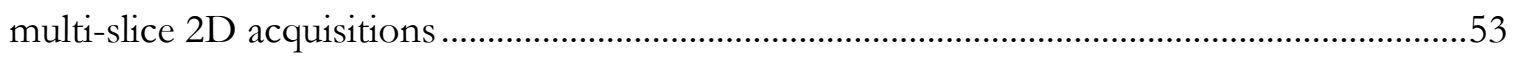

Table 2: Average flow rate measured through the phantom (averaged over 85 slices - 25 $\mathrm{cm}$ of length along the phantom) $-5 \mathrm{~cm}$ proximal to throat to $20 \mathrm{~cm}$ distal to throat. Re number is measured at the throat of phantom. .55

Table 3: Acquisition parameter for Cartesian PC MRI and UTE-PC MRI using stack of star trajectories

Table 4: Comparison of flow measurement between 3-D UTE and conventional PC MRI in 4 normal volunteers. Quantities have been averaged for the right CCA

Table 5: Mean square error (MSE) in flow measurement using 3-D UTE-PC MRI after phase error correction compared to conventional PC MRI

Table 6: Acquisition parameter for conventional 3D PC MRI and 3D UTE-PC MRI

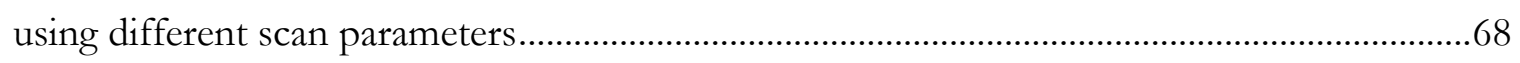

Table 7: Flow assessment in carotid artery using conventional 3D PC MRI and 3D UTE PC MRI with various scan parameters

Table 8: Comparison of 4D Cartesian flow and 4D spiral flow in 12 slices in right and left CCA and 20 slices in right and left ICA in four volunteers. The quantities are percentage of discrepancy in spiral technique relative to Cartesian technique .79 
Table 9: Peak flow rates, Reynolds numbers at inlet and throat of the stenosis and other scan parameters for pulsatile and steady flow regimes as well as scan times for conventional 4D flow and 4D UTE flow MR imaging of pulsatile flows. For 4D UTE sequence, 50\% radial sampling rate was used. The scan times for steady flows were reported in Table 10. The Shelley pump was used. . .88

Table 10: Steady flow measurement averaged for 12 axial slices distal to throat of the stenosis using conventional 4D flow MRI and 4D UTE flow MRI with various sampling rates and at different flow rates. Table entries are mean \pm standard deviation (across the slices). The Shelley pump was used.

Table 11: Pulsatile flow measurement averaged for 3, 2, and 12 slices located proximal, at the throat, and distal to throat of the stenosis (Figure 3) using conventional 4D flow MRI and 4D UTE flow MRI at different flow rates. Table entries for measured peak systolic flow are mean \pm standard deviation (across the slices). RRMSE is the Relative Root Mean Squared Error between prescribed pulsatile flow and the measured flow (Eq.). 101

Table 12: Mean flow (over the entire cardiac cycle) at each slice along the phantom using conventional 4D flow MRI and 4D UTE flow MRI for the flow-on and flow-off experiments and the final result after phase error correction through subtraction of flow-off data from flow-on data. The unit for measurement is $\mathrm{ml} / \mathrm{s}$. The LB pump was used......... 108

Table 13: Measured parameters using conventional 4D flow MRI, 4D UTE flow MRI, and Doppler echo technique in nine patients. 125

Table 14: Comparison between different MR flow measurement techniques utilized or developed in this dissertation. All the imaging parameters were considered the same at flow rate $300 \mathrm{ml} / \mathrm{s}$ which required a Venc of 500 which is assumed the same for all methods and for all 3 directions. The UTE techniques have 75\% sampling rate of radial k-space line and 
the spiral techniques used $4 \mathrm{msec}$ readouts and 30 interleaves. \#Encoding in the table refers to number of flow encoding directions during one scan. .................................................... 143 


\section{INTRODUCTION}

Assessment of blood flow is key to diagnosis of disease in the cardiovascular system. Phase-contrast (PC) MRI is a reliable non-invasive technique for characterization and quantification of blood flow [1, 2]. PC MRI is based on the fact that flowing blood induces phase shift in MR signal which is proportional to velocity of flowing blood. In standard MR imaging, signal intensity is the basis for image reconstruction and the acquired image is called the magnitude image. However, in PC MRI the phase of spins inside each voxel is the basis for image reconstruction and the reconstructed image in this case is referred to as the phase image. A flow sensitive gradient is applied during acquisition and consequently the image phase correlates to velocity of flowing spins. The reconstructed image in this case is called phase velocity map or phase contrast image wherein the phase image is mapped to describe the spatial distribution of velocity across the image. PC MRI is in abundant clinical use for quantification of blood velocity and flow. PC MRI, however, is not accurate in cases where there is high speed flow, for example in the setting of atherosclerotic disease and vascular or valvular stenosis. For example, vascular stenosis cause disturbed blood flow, flow recirculation, turbulence, flow fluctuation, and a jet distal to the narrowing.

With conventional MR imaging techniques, high speed flows result in signal loss distal to the stenosis and cause miscalculation in flow quantification [3]. Several approaches have been developed to potentially reduce the signal loss in PC MRI [4-7]. However, a reliable flow measurement technique in the presence of turbulence remains elusive. One important approach that appears to yield significant improvements and to correct the signal loss 
involves reduction of the echo time (TE) and gradient duration [8-11]. Reducing the TE decreases the impact of turbulent fluctuation velocity, intravoxel dephasing and the subsequent signal loss. This approach results in higher signal to noise ratio and more reliable estimation of disturbed and jet flows in PC MRI since a shorter TE will ameliorate the effect of intravoxel dephasing caused by random fluid mixing.

Standard Ultra Short TE (UTE) techniques, in general, however suffer from phase errors due hardware imperfections, specifically gradient channel delays. This problem becomes quite pronounced in flow measurement wherein the phase information of image needs to be used for quantification of flow. Previously, a 2-D UTE-PC technique was investigated for measurement of blood flow through the aortic valve by O’Brien et al. [12]. In addition to the inherent limitations of the UTE technique, the previously proposed 2-D UTE-PC technique by O'Brien et al. has other issues of concern including underestimation of low flow rates, sensitivity to scanner imperfections, and in particular, $\mathrm{B}_{0}$ inhomogeneities resulting in phase errors and velocity miscalculations, and limitations on the minimum slice thickness.

This dissertation is structured as follows:

In Chapter 2, the physiology of blood flow and anatomy of vascular wall and atherosclerotic disease are briefly described with more emphasis on carotid artery and aorta as typical sites for disturbed and turbulent flow. Various flow regimes and disturbed and turbulent blood flows are also explained in this Chapter.

In Chapter 3, basic physics of MRI and image formation are described. The UTE technique and related trajectories for 3-D acquisition are also discussed. In Chapter 4, flow imaging techniques and flow quantification are described in detail. 
In Chapter 5, conventional techniques for disturbed and turbulent flow quantification are introduced and their advantages and disadvantages are discussed. The proposed UTE-PC technique is explained and a phase error correction method is proposed.

Chapter 6 demonstrates results from a static no-flow phantom, steady flow in a stenotic phantom, and in-vivo study in carotid artery of normal subjects.

In Chapter 7, the effect of various parameters including Venc, gradient strengths and slew rates, as well as k-space sampling density are assessed using 3-D UTE flow MRI. Chapter 8, introduces a new 4D flow MRI technique based on spiral trajectory and describes advantages of the proposed technique over conventional 4D flow MRI. In Chapter 9, a novel 4D flow MRI technique based on UTE acquisition is introduced and the results are compared with conventional 4D MRI technique in a stenotic phantom with various steady and pulsatile flow rates. Chapter 10, reports on the results of a correlative patient study which included subjects with aortic stenosis using different imaging techiques including 4D UTE flow MRI [13], conventional 4D flow MRI, and Doppler ultrasound. Finally, in Chapter 11 conclusions and future research directions are outlined. 


\section{PHYSIOLOGY OF BLOOD FLOW, ANATOMY OF THE VESSEL WALL, AND ATHEROSCLEROTIC DISEASE}

\subsection{Introduction}

The main function of cardiovascular system is transporting nutrients throughout the body. The heart, as the engine of the system, pumps the blood through a complex network and tubes, so called vessels. These vessels include arteries, arterioles, capillaries, and veins and distribute the blood and nutrients to all the organs and transfer wastes to exit from the human body [14]. Vessels such as arteries and veins are adaptable to various blood flows and pressures to meet the variations in hemodynamic demands.

Cardiovascular arteries and blood flow have been widely investigated in normal and diseased physiologic conditions. In this Section, the physiology of blood flow, anatomy of vessel wall, and atherosclerotic disease are described.

\subsection{Physiology of blood flow}

Blood is comprised of various components such as protein, red blood cell, white blood cell, lipoproteins, and ions by which nutrients and wastes are transported. Red blood cell is one of the main ingredients of blood and includes approximately $40 \%$ of blood [15]. Red blood cells affect the viscosity of blood due to their semisolid particles and lead to 4 time 
higher viscosity compared to water. Viscosity defines the tendency of a fluid to resist flow.

Viscosity of blood in low flow rate is higher because red blood cells stick together and

aggregate in the middle of vessel.

The heart pumps the blood throughout the vessel in a cyclic manner. This cyclic pumping is divided to systolic and diastolic phases [14]. In the systolic phase, the blood pumps out of the heart and transfers to organs. In this phase, blood flow and pressure are higher in arteries. In the diastolic phase, the heart is in its rest phase and does not pump blood. Blood flow and pressure drop in arteries in the diastolic filling phase when arterial and venous blood return to the heart. In general, blood flow is unsteady/ pulsatile during the

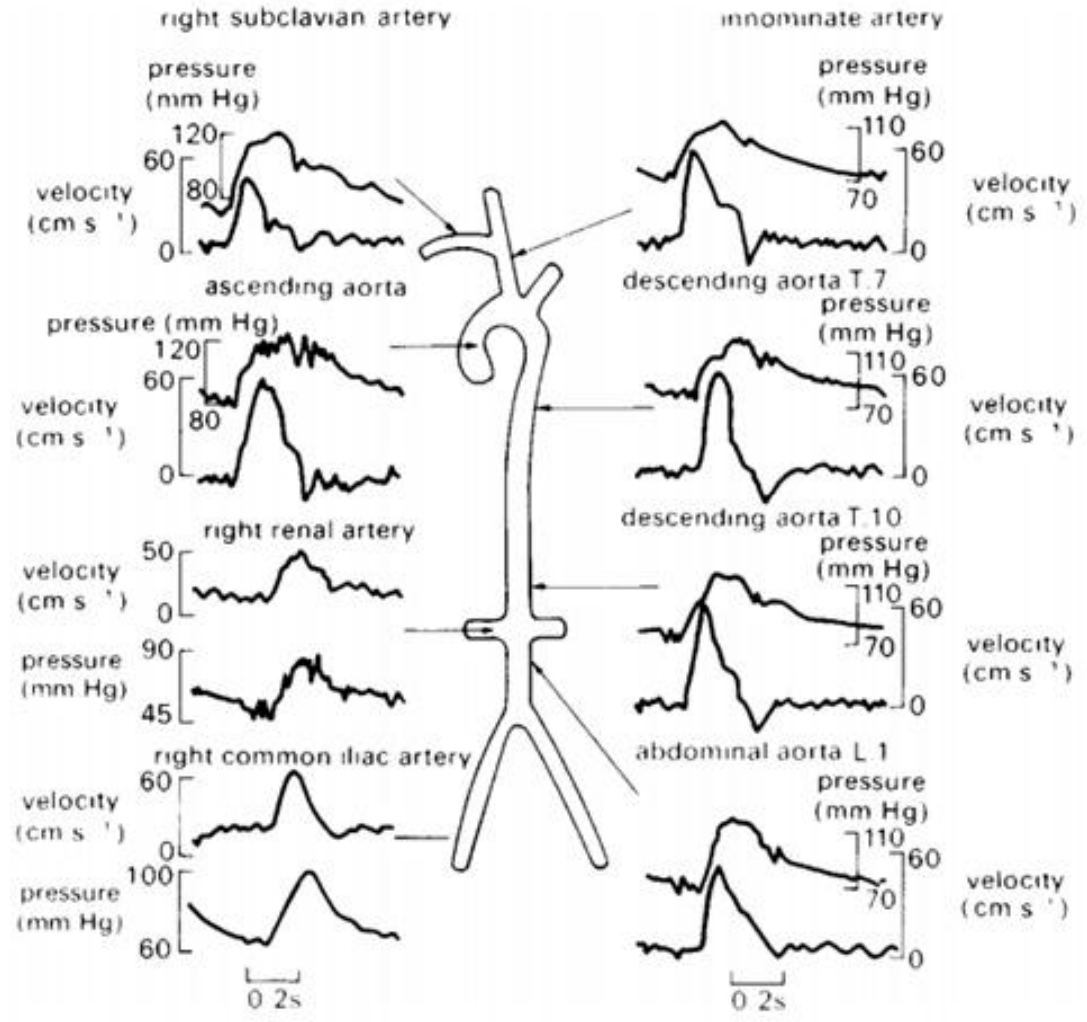

Figure 1: A schematic of blood velocity and pressure in the vascular tree. The blood velocity decreases as one move away from the heart while the pressure increases. Taken from [16]. 
cardiac cycle and varies in arteries and veins with respect to cardiac phase. Figure 1 exhibits the blood velocity and pressure in arterial system.

The blood leaves the heart in a large artery called the aorta. The pulsatile blood pressure in aorta is high in systole and becomes small but does not become zero during diastole. On the other hand, blood flow can be zero and even negative in diastole is some arteries such as the external carotid artery and the femoral artery [15]. These arteries have high downstream resistance during rest and the flow is on and off with each cardiac cycle. Internal carotid artery and renal artery, on the other hand, can have a higher flow during diastole with low downstream resistance.

\subsubsection{Aorta}

Figure 2(a) demonstrates the proximal aorta and its branches. Aorta is the largest artery in

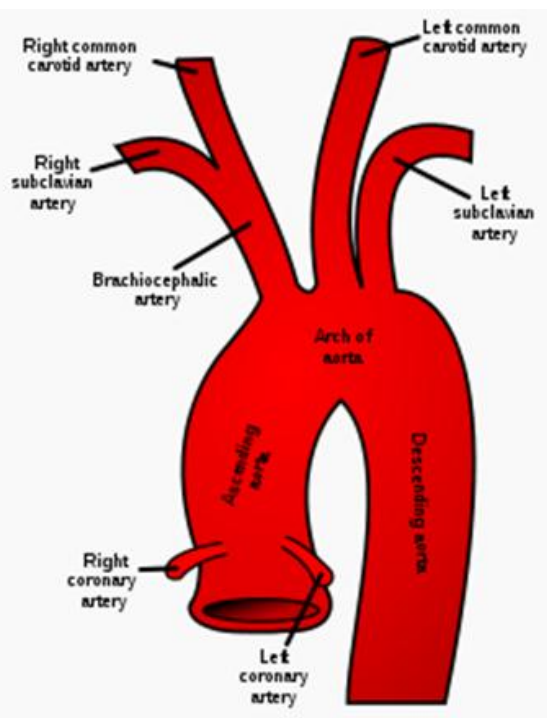

(a)

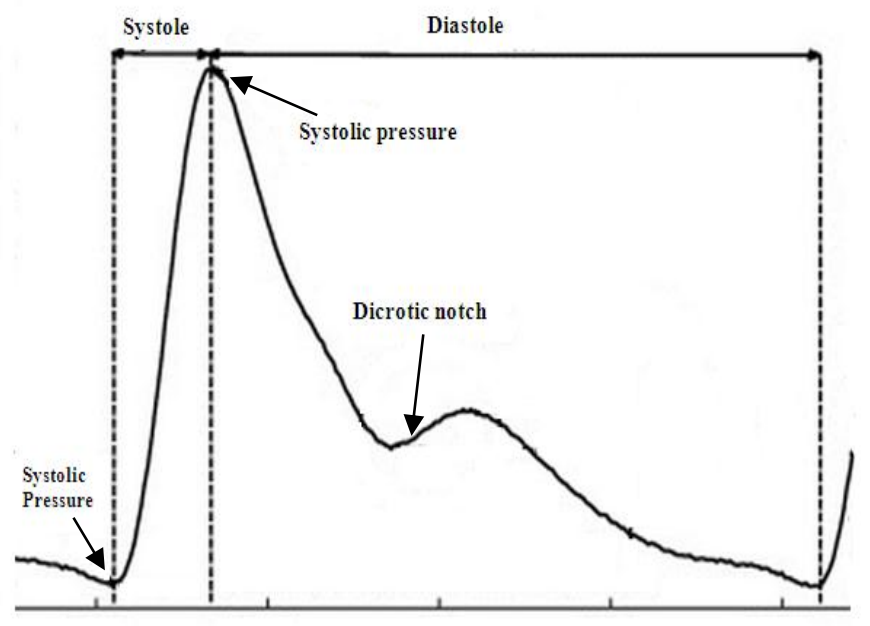

(b)

Figure 2: A schematic of aorta and its main branches (taken from [17])(a), and arterial pressure curve including systole, dicrotic notch and, diastole (taken from [18])(b). 
body, originating from the left ventricle and distributing the blood throughout the body. Aorta is generally divided into five Sections: ascending aorta, aortic arch, thoracic aorta, descending aorta, and abdominal aorta [1]. Aorta travels upwards in the beginning and is called ascending aorta. In the aortic arch few branches go to upper body and head. After aortic arch, the vessel turns downwards, where it is called the descending aorta and is divided to several branches supplying various organs in the mid and lower body. Aorta has the highest blood pressure in the circulation system. Unlike other regions of body, turbulent flow in the aorta is very likely and Reynolds number is higher. Reynolds number distal to aortic valve is approximately 4000 which is consistent with turbulent flow [15].

The pulsatile feature of blood flow generates a pulse waveform that propagates down the arterial tree and at bifurcations bounces back to the origin of aorta. The dicrotic notch on aortic pressure valve is created due to this pulse wave return as this wave push on the aortic semilunar valve (Figure 2) [19]. The stiffness in aorta is one of the common diseases in aged people and leads to faster propagation of this pulse wave and also faster return of this waveform through semilunar valve before closing of this valve. Pulse waveform and velocity measurement may be used to determine arterial stiffness [20].

Aortic valve, with 3 leaflets, is located between left ventricle and the aorta. Aortic stenosis (AS) is a critically significant valvular heart disease and results in the valve not fully opening or being significantly narrowed. Typically, the condition develops due to calcium deposits around the valve and occurs in the elderly. Presence of an AS results in the need for the left ventricle to increase the pressure in order to pump the blood into the aorta. Over time, the additional work required by the left ventricle results in increased thickness for the ventricular wall and chest pain. Clinical classification of AS includes mild, moderate, severe, and critical. 


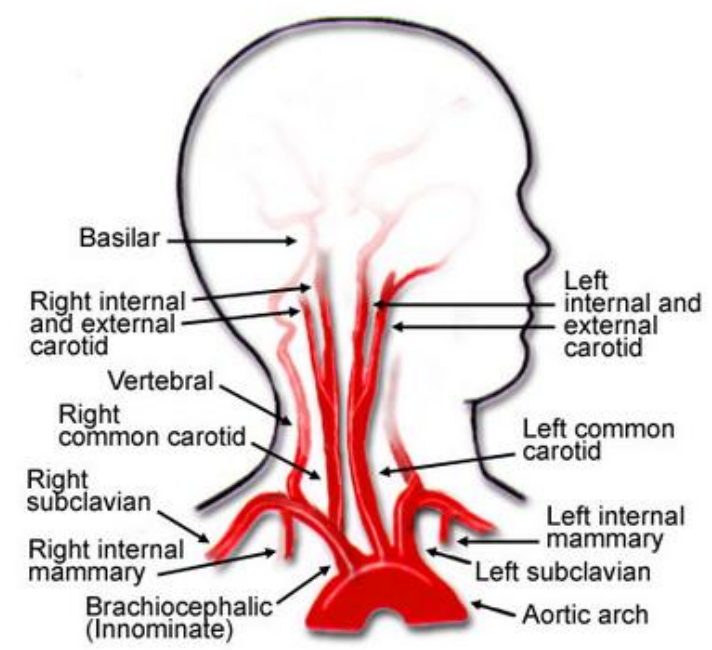

Figure 3: Carotid artery and its branches in left and right side of neck (taken from [22]).

Mild stenosis are usually asymptomatic and symptoms occurs in patients with severe or critical valvular stenosis. The main symptoms are syncope, anginal chest pain, and dyspnea [21].

\subsubsection{Carotid artery}

The carotid artery is an artery which branches from the aorta and supplies the head and neck (Figure 3). It is referred to as the common carotid artery as a pair of them travel upwards on the right and left side of the neck. Each of the right common carotid artery (RCCA) and the left common carotid artery (LCCA) are divided to internal carotid artery (ICA) and external carotid artery (ECA) at carotid bifurcation [23]. Carotid bifurcation is one of the main sites for development of atherosclerotic disease due to the atypical geometry.

According to the American Heart Association, up to 25\% of strokes in the U.S. are caused by atherosclerosis of the Carotid arteries through atheroembolization. 
Figure 4 demonstrates the flow pattern in a glass model of carotid bifurcation illustrating various flow streams. The main flow travels upward along the flow divider along the posterior wall of the internal carotid sinus[15]. The Secondary vortices which are counterrotating travel upward through the common carotid in a separation area. In The region of

internal carotid sinus wall mean wall shear stress (tangential resistive fluid force due to viscous effects on the endothelium) is low and oscillation in flow appears in this area which makes this region more prone to atherosclerotic plaques. The mean Reynolds number (dimensionless parameter representing the balance of inertial to viscous fluid forces) is approximately 300 at this bifurcation.

\subsection{Anatomy of the vessel wall and atherosclerotic disease}

To better understand the disturbed and turbulent blood flow here the anatomy of vessel wall and atherosclerotic disease are discussed. Arteries, veins and capillaries do not have the same anatomy. Arteries and veins are surrounded with layers of muscles to provide the

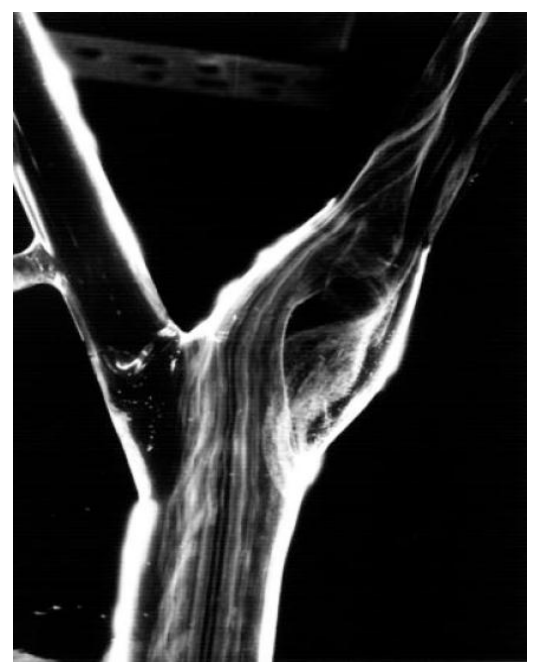

Figure 4: Visualization of flow in a glass model of carotid bifurcation[15]. 


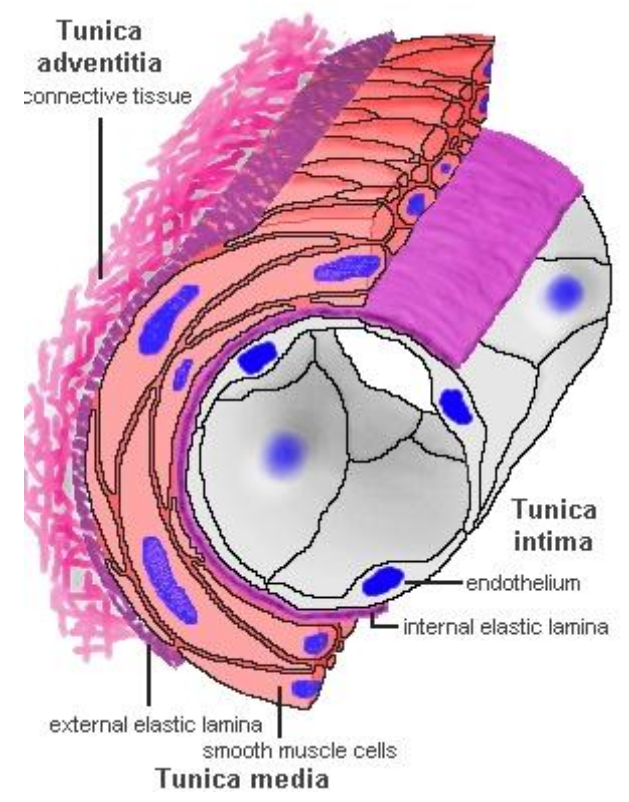

Figure 5: The three layers of vessels walls (taken from [24]).

possibility of contraction. These muscles are thicker in arteries, particularly, in aorta which leaves the heart and comprises cardiac muscle fibers in few first inches after leaving the heart. Arteries and Veins walls contain three layers of coats called tunics: Tunica intima, tunica media, and tunica adventitia [25]. These layers are not visible in capillaries. Tunica intima is the innermost layer which is in direct contact with blood. This layer lines the

interior layer of vessel wall or lumen. The tunica media is the middle layer containing smooth muscles to change the diameter of vessel and is controlled by the sympathetic nervous system. The tunica adventitia is the external layer which mainly consist collagen and elastic fibers. The tunica adventitia combines with the connective tissue surrounding the vessel. Figure 5 shows the anatomy of the vessel walls and its three layers. Recent investigations shows that for unknown reasons the blood cells attack a layer of vessel wall cells (endothelium) in arteries. This phenomena cause inflammation in vessel wall leading to 
formation of plaques attached to vessel walls in Tunica intima [26, 27]. These plaques generally consist of collagen, fat, and elastin.

Atherosclerotic disease is a condition that plaques and fatty materials accumulate in the vessel and a narrowing or hardening of vessel appears. Figure 6 illustrates an artery with Atherosclerotic disease due to plaque accumulation. Atherosclerotic disease mostly affects large and high- pressure vessels such as coronary, carotid, femoral, and cerebral arteries.

Plaques are composed of cholesterol, fat, calcium and other material in the blood. The accumulation of plaques and consequently narrowing in vessel can cause abnormal flow such as reduced blood flow, disturbed and turbulent blood flow distal to narrowing. This causes the limitation of oxygen-rich blood in the heart and other body organs.

\subsection{Flow regimes}

Blood flow is classified as two major regimes: 1- laminar flow and 2- turbulent flow [15]. Laminar flow, known also as streamline, occurs when the flowing particles in a parallel line
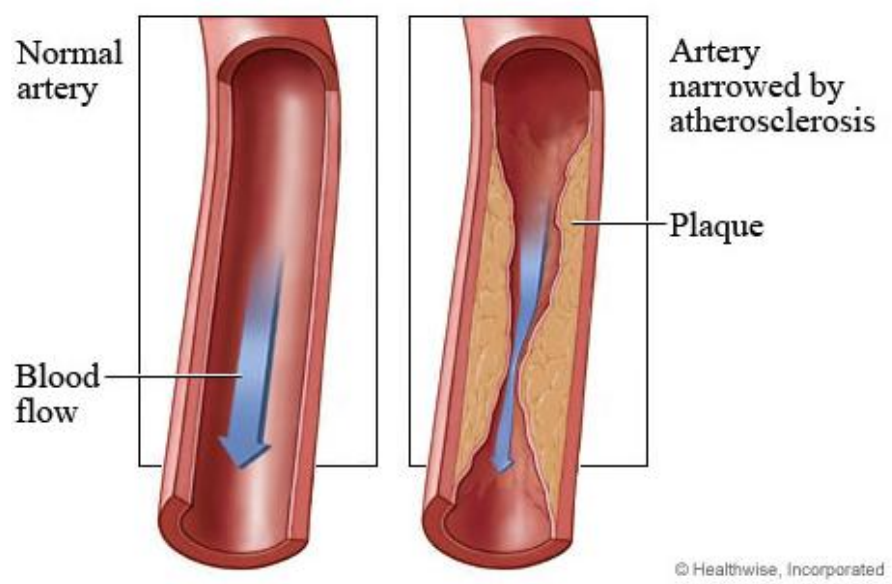

Figure 6: Narrowing in a blood vessel due to accumulation of plaque (taken from [28]). 
move without mixing between layers. At low velocities, fluid particles in laminar flow do not have whirling motion, eddies, or other motion perpendicular to the flow direction. The velocity profile in laminar flow is parabolic as shown in Figure 7 [29]. The mean velocity, V, is half of the maximum velocity in the center line of flow and local velocity value, $U$, can be calculated as

$$
\frac{\mathrm{U}}{\mathrm{V}_{\max }}=1-\left(\frac{\mathrm{r}}{\mathrm{R}}\right)^{2}
$$

where $\mathrm{R}$ is the radius of the pipe. On the other hand, turbulent flow occurs at higher velocity where irregular movement such as eddies, whirling and lateral mixing happens. The whirling and eddies in turbulent flow generally causes a rapid variation of flow at arterial bifurcations, vascular or valvular stenosis and/or coarctation. Similar to laminar flow, in turbulent flow, the velocity profile is parabolic but flatter across the core of blood flow. The local velocity value can be approximated with

$$
\frac{\mathrm{U}}{\mathrm{V}_{\max }}=1-\left(\frac{\mathrm{R}}{\mathrm{r}}\right)^{\mathrm{n}}
$$

where $\mathrm{n}$ depends on friction factor [31]. The mean velocity, $\mathrm{V}$, is very close to maximum velocity and the Equation (2) which is based on power law is a good description of the mean shape of velocity profile in turbulent flow. Figure 7 illustrates the cross-Sectional velocity

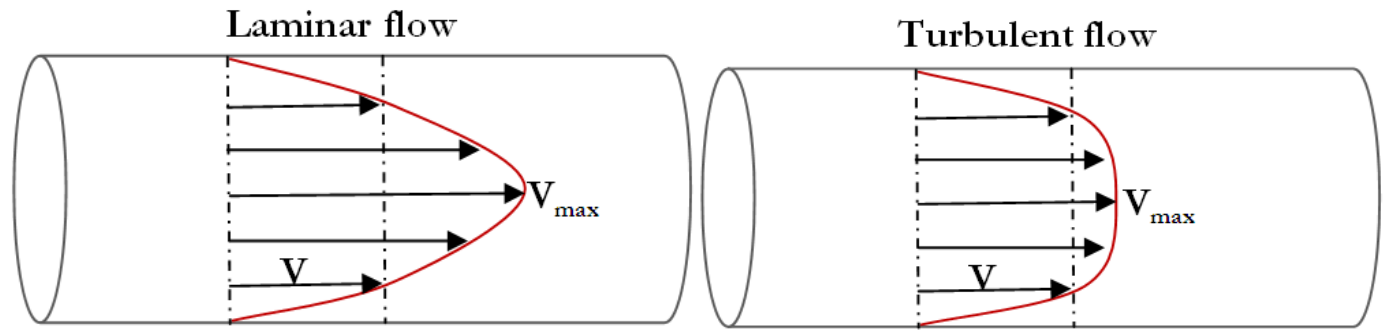

Figure 7: A schematic of laminar flow (left column) and turbulent flow distal to stenosis (right column). The bottom row shows the velocity profile for these flows [30]. 
profiles of laminar and turbulent flows in a pipe.

Fluid flow can be classified as steady flow and unsteady or pulsatile flow. When the fluid properties at a point do not varies over time, the flow is considered to be steady. Pulsatile flow, on the other hand, is defined as intermittent propagation of fluid with a time varying flow function [32]. In the cardiovascular system, pulsatile blood flow results in velocity variation in vessels as a function of phase of the ECG cycle. Additionally, velocity variations correspond to the distance between the vessels and the heart as well as timing in cardiac phase. This time varying flow leading to rapid change in blood velocity causes difficulty in accurate blood flow quantification.

\subsection{Reynolds number}

Reynolds number [33] is a commonly used parameters in characterization of different flow regimes and can be used to determine the transition from laminar to turbulent flow [34]. This dimensionless quantity is associated with the ratio of inertial forces to viscous force and in a pipe flow is generally defined as

$$
\operatorname{Re}=\frac{\rho v D}{\mu}
$$

where $\rho$ is the density of the fluid $\left(\mathrm{kg} / \mathrm{m}^{3}\right), \mathrm{v}$ is the mean velocity of the object relative to the fluid (m/s), D is the diameter of the pipe (m), and $\mu$ is the dynamic viscosity of the fluid $(\mathrm{kg} /(\mathrm{m} \cdot \mathrm{s}))$. In general, low Reynolds numbers are associated with laminar flows and high Reynolds numbers represent non-laminar or turbulent flows. In vessels, Reynolds numbers lower than 2000 are associated with laminar flows with constant and smooth flow and higher than 2000 are associated with turbulent flow [35, 36]. In normal cardiovascular system, Reynolds number is less than 2000 and blood flow appears to be laminar. High Reynolds 
number flows generally appear distal to vascular or valvular stenosis and/or coarctations with chaotic eddies and flow patterns [15, 37]. In addition, Reynolds numbers between 2000 and 3000 are known as laminar-turbulent transitional flow.

\subsection{Disturbed and turbulent blood flow}

Numerous investigations have shown that disturbed and turbulent flow may be involved in pathophysiological processes and cardiovascular disease such as thrombosis [38], intravascular hemolysis in the region of prosthetic valves [39], cardiac murmur [40], thickening in the region of prosthetic valves and coronary arteries [41], and development of atherosclerosis [42]. One known factor for producing turbulent blood flow is acceleration and deceleration of pulsatile flow during systole and diastole [43]. Another important factor is pulse frequency of blood flow whereas for example, short pulse frequency results in a short time for deceleration period to be able to develop a turbulent flow, since turbulent flow needs enough time to occur.

As previously mentioned, turbulent flow may appear at arterial bifurcations, vascular or valvular stenosis and/or coarctation. One of the main sites of turbulent flow is at the ascending aorta specifically distal to a diseased aortic valve [37]. Another demonstrated site of disturbed and turbulent blood flow is arterial branches and bifurcations such as carotid bifurcation which are more prone to atherosclerotic plaques.

The most prevalent feature of turbulent flow is its noisy sound due to fluctuation in pressure [44]. For many years, the stethoscope has been a very common tool for diagnosis of cardiovascular diseases such as aortic valve stenosis. In previous studies, invasive and noninvasive methods for turbulent flow quantification in cardiovascular system have been 
investigated. Catheter hot-film anemometry or perivascular Doppler ultrasound have been used to assess turbulent flow in humans [37, 45, 46]. Magnetic resonance imaging is now a standard technique for blood flow quantification and is commonly used in clinical practice. However, this technique is not very accurate for turbulent flow quantification due to velocity fluctuation and intravoxel dephasing leading to signal loss and flow measurement errors [9, 47]. Many investigations have been done to visualize and quantitatively measure the turbulent flow using MRI [48-57]. However, an accurate technique to assess turbulent flow has not been developed. In this project, a new technique based on MRI for quantification of disturbed and turbulent flows is investigated. 


\section{MAGNETIC RESONANCE IMAGING}

\subsection{Proton magnetic resonance and net magnetization}

The core of all atoms is the nucleus which includes positively charged and neutral particles that account for the bulk of the atomic mass. All nuclei that possess either an uneven mass number (proton + neutron) or uneven atomic number (number of protons) possess angular momentum and therefore a characteristic so called spin quantum number, I, greater than zero. The spin characteristic of the nucleus includes a magnetic field with a magnitude and direction represented by the magnetic moment (Figure 8). Normally, the magnetic moments of nuclei are randomly oriented due to the motion produced by thermal energy, resulting in a zero ensemble average. By applying an external static magnetic field, the magnetic dipoles orient to either parallel or anti-parallel to the direction of static field [59]. Summing all of the vectors of magnetic moments in a collection of nuclei in both parallel and anti-parallel directions results in net magnetization of the tissue in presence of an

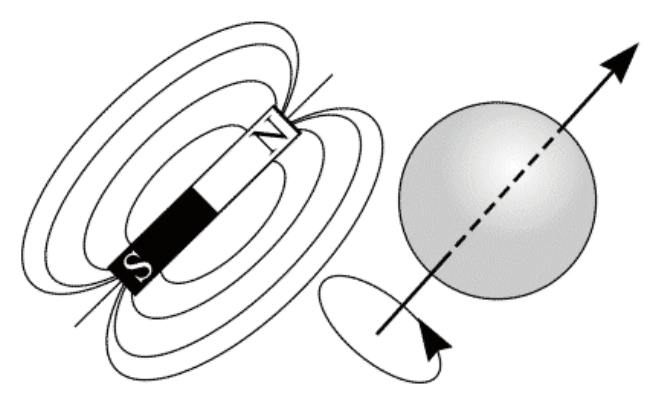

Figure 8: Spinning nuclei induces a magnetic moment which behaves like a bar magnet [58]. 
applied magnetic field. This net magnetization is parallel to the applied field due to slight excess of spins in the parallel state.

In the presence of an applied magnetic field, the spin vectors of the nuclei experience a torque which causes them to rotate around the axis of the applied field with a precise frequency. This rotation around the applied field is called Larmor precession which depends on the specific physical characteristic of the isotope and the strength of magnetic field as

$$
\omega=\gamma \mathrm{B}_{0}
$$

where $\omega$ is larmor frequency $(\mathrm{MHz}), \gamma$ is gyromagnetic ratio which is a constant of proportionality and is unique for different NMR-active nuclei, and $\mathrm{B}_{0}$ is the applied magnetic field strength (Tesla, T) [60]. Since due to presence of water and fat, proton $\left({ }^{1} \mathrm{H}\right)$ is the dominant nuclei in body, this nucleus is primarily used in MR imaging. The gyromagnetic ratio for proton is $42.58 \mathrm{MHz} / \mathrm{T}$.

\subsection{Resonance phenomena and signal generation}

The net magnetization, $M_{0}$, in its equilibrium state is parallel to $B_{0}$ and as such does not induce a current in a receiver coil, as defined by Faraday's law of induction [61]. In order to obtain a signal in receiver coil, the spin system is irradiated with a RF pulse with the same Larmor frequency as the proton. This RF pulse causes a deflection of the net magnetization from its equilibrium orientation leading to rotation of net magnetization around the static magnetic field with the Larmor frequency [59]. Figure 9 demonstrates the precession of net magnetization around the $B_{0}$ field by applying the $B_{1} R F$ pulse. $B_{y}$ applying $B_{1}$ field the net 


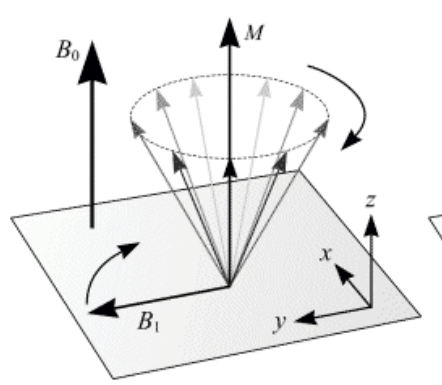

(a)

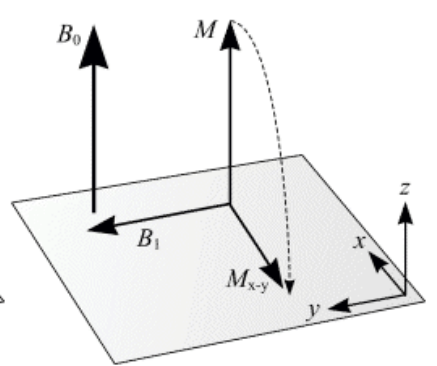

(b)

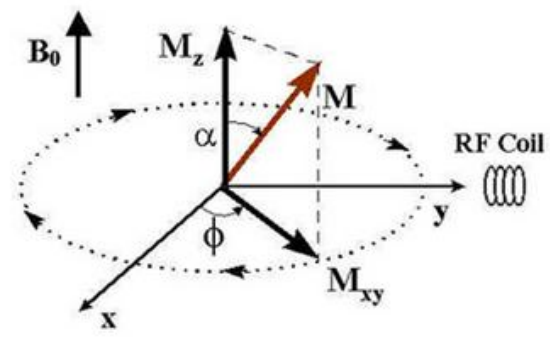

(c)

Figure 9: Precession of net magnetization $M$ around the $B_{0}$ field by applying the $B_{1} R F$ pulse (taken from [58]).

magnetization rotates away from vertical direction toward X-Y plane. The angle of rotation is a function of the amplitude and duration of $\mathrm{B}_{1}$ field as expressed by:

$$
\theta=\gamma B_{1} t
$$

where $\theta$ is the angle of rotation or flip angle, $B_{1}$ is the amplitude of the RF pulse, and $t$ is the duration of the RF pulse [59]. Figure 9(c) shows that as net magnetization (M) after rotation from equilibrium direction and has two components in the transverse plan $\left(\mathrm{M}_{\mathrm{xy}}\right)$ and longitudinal plane $\left(\mathrm{M}_{z}\right)$.

Immediately after termination of the RF pulse, the relaxation process starts in which the net magnetization returns back to equilibrium position. This relaxation includes returning of both transverse $\left(M_{x y}\right)$ and longitudinal $\left(M_{z}\right)$ magnetization to their equilibrium state. In the equilibrium state, $\mathrm{M}_{\mathrm{z}}$ becomes equal to $\mathrm{M}_{0}$, which is proportional to spin density, and $\mathrm{M}_{\mathrm{xy}}$ disappears. Figure 10 demonstrates the longitudinal and transverse magnetization during relaxation. These magnetizations relax back to their original values in an exponential fashion 

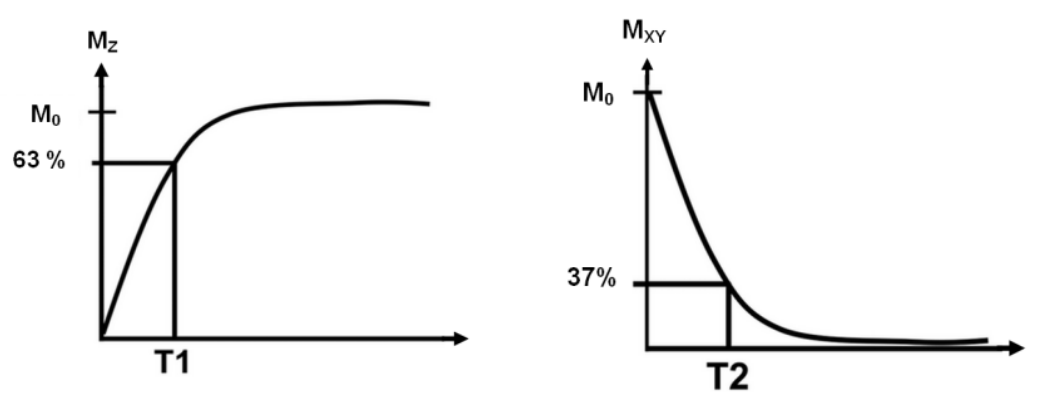

Figure 10: longitudinal and transverse magnetization during relaxation. T1 and T2 are independent and T2 is always shorter than T1 [62].

with time constant $T_{1}$ and $T_{2} . T_{1}$ is the time-constant describing the time required for longitudinal magnetization to return to $63 \%$ of its thermal equilibrium value [62]. The physical basis for T1 relaxation involves spins losing their energy to the lattice environment. Immediately after excitation by RF pulse, the spins precess coherently, creating a transverse component of the net magnetization in XY plane. However, interaction between spins creates random local magnetic field variation leading to fluctuations in the precessional frequency of protons and loss of phase coherence. Consequently, the magnitude of the transverse magnetization $\mathrm{M}_{\mathrm{xy}}$ decays to $37 \%$ of its original value in a time equal to $\mathrm{T}_{2}$ and finally decays completely [62]. Figure 11 shows how the spins begin to lose phase coherence, some spins gain a few degrees on the net magnetization, while others fall a few degrees behind, over time all pointing in random directions.

The receive coil located perpendicular to equilibrium net magnetization can be used to detect the signal provided by transverse magnetization decay. This detected signal can be expressed using Bloch Equation as follows [63]:

$$
\frac{\mathrm{dM}}{\mathrm{dt}}=\gamma \mathrm{M} \times \mathrm{B}-\frac{\mathrm{M}_{\mathrm{x}}}{\mathrm{T}_{2}} \hat{\mathrm{x}}-\frac{\mathrm{M}_{\mathrm{y}}}{\mathrm{T}_{2}} \hat{\mathrm{y}}-\frac{\mathrm{M}_{\mathrm{z}}-\mathrm{M}_{0}}{\mathrm{~T}_{1}} \hat{\mathrm{z}}
$$

where $T_{1}$ and $T_{2}$ are the longitudinal and transverse relaxation times. 

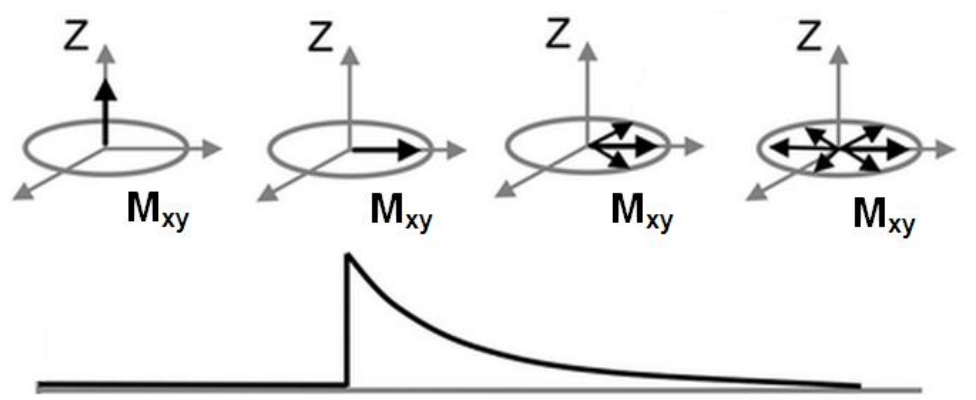

Figure 11: $\mathrm{T}_{2}$ relaxation process. As spin start to precess, the magnitude of its $\mathrm{M}_{\mathrm{xy}}$ component is reduced [64].

There are two mechanisms leading to loss of phase coherence and decay of transverse magnetization. The first involves spins experiencing slightly different magnetic field due to their interactions with neighboring nuclei. The second results from hardware imperfections and $\mathrm{B}_{0}$ inhomogeneity. Figure 12 illustrates the exponential decay in detected signal due to pure $T_{2}$ relaxation and the combined effect including $B_{0}$ inhomogeneity leading to $T_{2}^{*}$ which is shorter from $\mathrm{T}_{2}$.

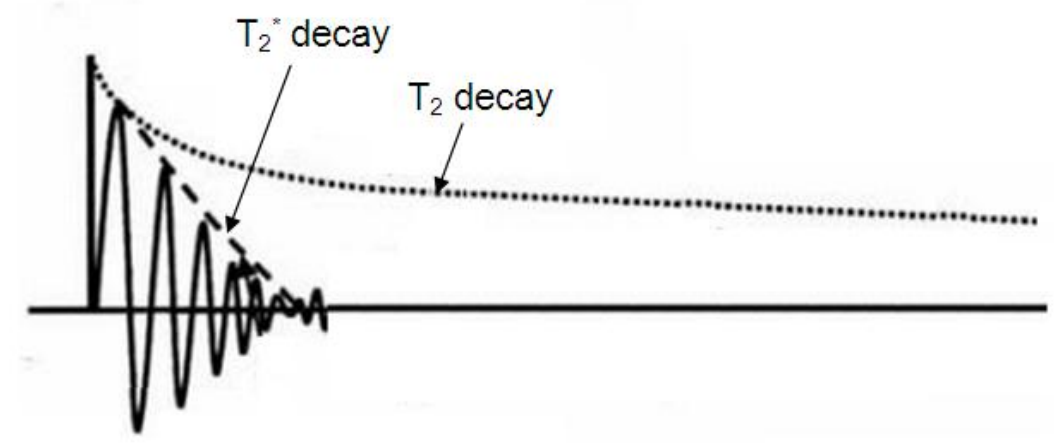

Figure 12: Exponential decay in detected signal. Due to $\mathrm{B}_{0}$ inhomogeneity the decreasing of signal is accelerated such that $\mathrm{T}_{2}^{*}$ is significantly shorter than $\mathrm{T}_{2}[58]$. 

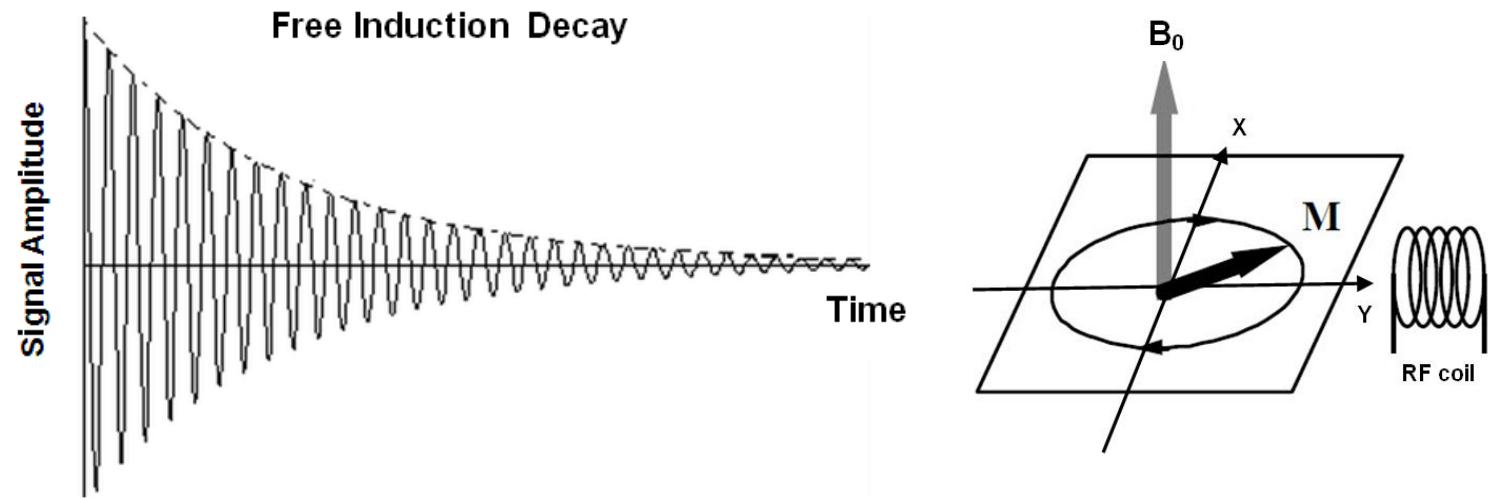

Figure 13: A typical free induction decay signal and measurement of a time based signal using RF receive coil [64].

\subsection{Free Induction Decay (FID)}

As it was described to this point, a signal can be acquired in transverse plane by application of a static magnetic field $\mathrm{B}_{0}$ and second $\mathrm{RF}$ field $\mathrm{B}_{1}$. The measurable signal can be described by its temporal component or by their frequency component including amplitude and phase. In general, the associated frequency for a time-dependant signal can be mathematically represented by Fourier transformation.

In $\mathrm{MR}$ imaging, the net magnetization $\mathrm{M}_{0}$ can be rotated to transverse plane by application of a 90 degree RF pulse. This rotation induces a signal in a receive coil after terminating the RF pulse [60]. This signal, which is the result of the free precession of the net magnetization in the transverse plane, is known as the Free Induction Decay (FID) since it gradually decays due to relaxation mechanism as illustrated in Figure 13.

This FID signal oscillates at Larmor frequency and its amplitude starts from an initial value which is proportional to the density of observed protons in the sample being measured. The amplitude decreases exponentially with $\mathrm{T}_{2}^{*}$ effective transverse relaxation time 
constant. By measuring this FID signal and employing Fourier transformation the frequency-based MR spectrum is obtained.

For the described FID signal, the RF pulse and FID signal detection take place in one uninterrupted sequence. However, it is sometimes advantageous to collect signals after disappearance of FID signal using techniques which are known as spin echo and gradient echo.

\subsection{Image formation}

When a subject is placed in an MR system, the protons align along the static magnetic field and precess with the proton Larmor frequency. When RF Field is applied perpendicular to the static magnetic field the protons are excited and precess into phase. MR signal is detected immediately after RF pulse termination. There is a problem with the approach however in that all the protons' resonance frequency are identical and therefore the detected signal in the receive coil does not contain any spatial information.

\subsubsection{Slice selection}

In order to relate the detected signal to spatial positions of the protons which contribute to signal generation, two operations need to be performed: selection of a slice of interest and spatial encoding of the information. By adding another gradient to provide a linearly varying magnetic field with position, the main magnetic field would then depend upon position. If the volume is then exposed to an RF pulse with a narrow range of frequency, only those protons in the thin slice with the same Larmor frequency as RF pulse would be excited. This

operation is known as slice selection. In general, slice selection involves application of 
gradients in three directions and an RF pulse with the same range of frequency as the slice of interest [62]. The Equation for slice of interest can be written as

$$
\mathrm{f}_{0}=\frac{\gamma}{2 \pi}\left(\overrightarrow{\mathrm{G}}_{\mathrm{ss}} \cdot \overrightarrow{\mathrm{r}}+\mathrm{B}_{0}\right)
$$

where $\vec{G}_{\mathrm{ss}}=\left(\mathrm{G}_{\mathrm{x}}, \mathrm{G}_{\mathrm{y}}, \mathrm{G}_{\mathrm{z}}\right)$ is a combination of gradients in three directions and $\vec{r}=(x, y, z)$ is the position of slice of interest. The slice thickness of slice of interest is proportional to two parameters [65]: the bandwidth of the RF pulse $\left(\Delta \mathrm{w}_{\mathrm{s}}\right)$, and the amplitude of magnetic field gradient $\left(\left|G_{s s}\right|\right)$ :

$$
\mathrm{T}=\frac{2 \Delta \mathrm{w}_{\mathrm{s}}}{\gamma\left|\mathrm{G}_{\mathrm{ss}}\right|}
$$

\subsubsection{Spatial encoding}

If a second magnetic gradient, $G_{x}$, is applied orthogonal to the slice select gradient while receiving signal, the frequency of detected signal would be related to the its position along the $G_{x}$ gradient. The longer the frequency encoding gradient is left on, the higher the frequency components of the NMR signal that are swept. This gradient is known as readout gradient or the frequency encoding gradient [60]. This gradient provides spatial information along $\mathrm{x}$-axis in a slice of interest. The amplitude of each frequency component in Fourier transform of detected signal for each position along the $\mathrm{x}$-axis is proportional to summed signal in the y direction. 
To encode the spatial information in $\mathrm{Y}$-axis another gradient is applied prior to readout gradient in an axis orthogonal to the readout and the slice select gradient. This gradient is called phase encoding gradient, $G_{y}$, and in combination with $G_{x}$ provides the spatial information in two dimensions [60]. During application of the readout gradient, $\mathrm{N}_{\mathrm{x}}$ data points are sampled in the readout direction.

\subsubsection{K-space trajectory}

If $G_{y}$ gradient is changed for $N_{y}$ times in y-direction, a matrix with $\mathrm{N}_{\mathrm{x}} \times \mathrm{N}_{\mathrm{y}}$ is acquired. This matrix is known as k-space data set which is collected during data acquisition and is the frequency domain representation for an MR image. Figure 14 demonstrates a typical k-space date set. The horizontal axis, known as $K_{x}$, corresponds to the spatial frequency variable in the readout direction. The vertical axis, known as $\mathrm{K}_{\mathrm{y}}$, is the spatial frequency variable in the phase encoding direction. K-space variables can be written as

$$
\overrightarrow{\mathrm{k}}(\mathrm{t})=\frac{\gamma}{2 \pi} \int_{0}^{\mathrm{t}} \overrightarrow{\mathrm{G}}_{\mathrm{ss}}\left(\mathrm{t}^{\prime}\right) \mathrm{dt}^{\prime}
$$

where $\overrightarrow{\mathrm{G}}_{\mathrm{sS}}$ is the combination of slice selection, phase encoding and frequency encoding

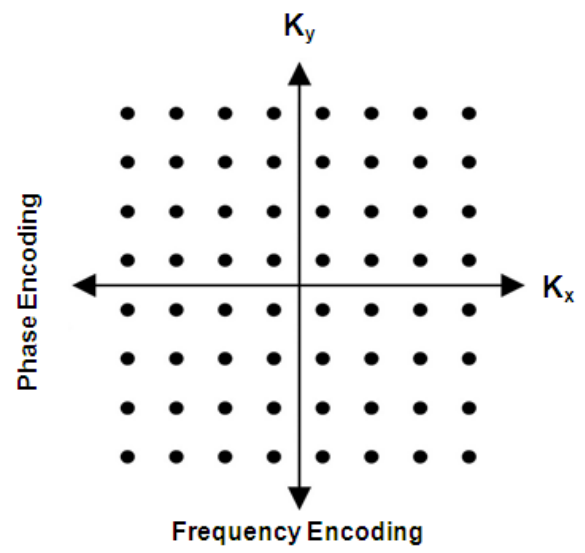

Figure 14: A typical k-space domain. $K_{x}$ denotes the direction of frequency encoding and Ky denotes the direction of phase encoding [66]. 
gradients [65].

The detected MR signal in receiver coils can be expressed as

$$
S(t)=\iint g(x, y) e^{-i \varphi(x, y, t)} d x d y=\iint g(x, y) e^{-i \gamma \int_{0}^{t} \vec{G}_{s s}\left(t^{\prime}\right) d t^{\prime} \cdot \vec{r}(t)} d x d y
$$

where $\mathrm{g}(\mathrm{x}, \mathrm{y})$ is the object being imaged. By substituting Equation (9) in (10)

$$
S(k(t))=\iint g(x, y) e^{-i 2 \pi\left(k_{x} \cdot x+k_{y} \cdot y\right)} d x d y
$$

where $\mathrm{S}(\mathrm{k}(\mathrm{t}))=\mathrm{G}\left(\mathrm{k}_{\mathrm{x}}, \mathrm{k}_{\mathrm{y}}\right)$ is the $\mathrm{k}$-space data. The final MR image can be acquired by application of inverse Fourier transformation to k-space data as follows:

$$
g(x, y)=\iint S(k(t)) e^{i 2 \pi\left(k_{x} \cdot x+k_{y} \cdot y\right)} d k_{x} d k_{y}
$$

Various techniques have been developed for playing out the gradient $\vec{G}_{s S}$ and to traverse the k-space. The most common technique is Cartesian trajectory which traverses the k-space linearly in a line by line fashion. Some other techniques which can be non-linear and/or nonparallel are spiral, and radial. Each technique has its own advantages and disadvantages.

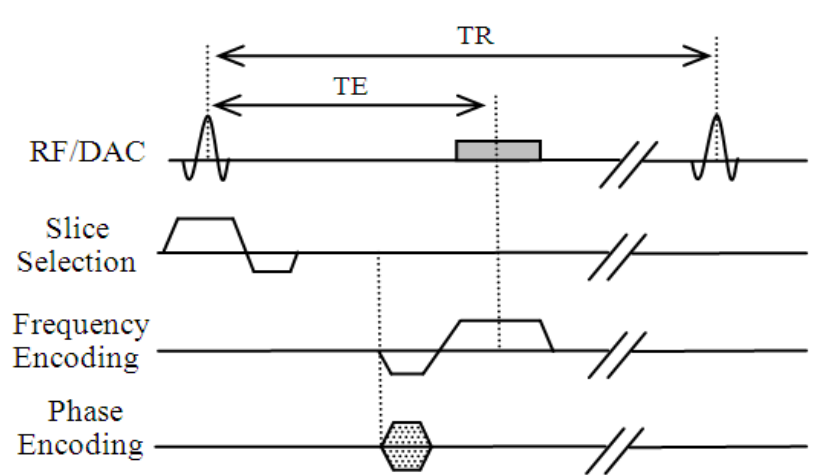

(a)

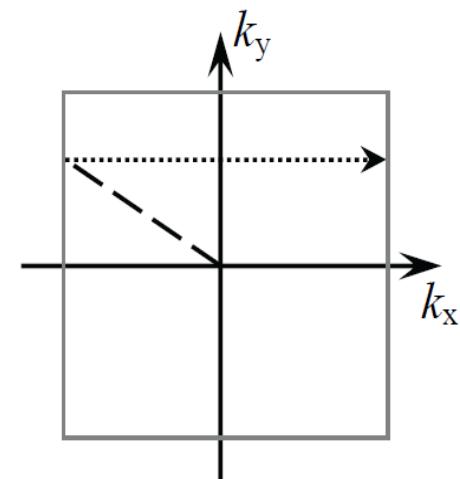

(b)

Figure 15: A schematic of gradient echo pulse sequence (a) which sample k-space lines in Cartesian fashion (b) $[65]$. 


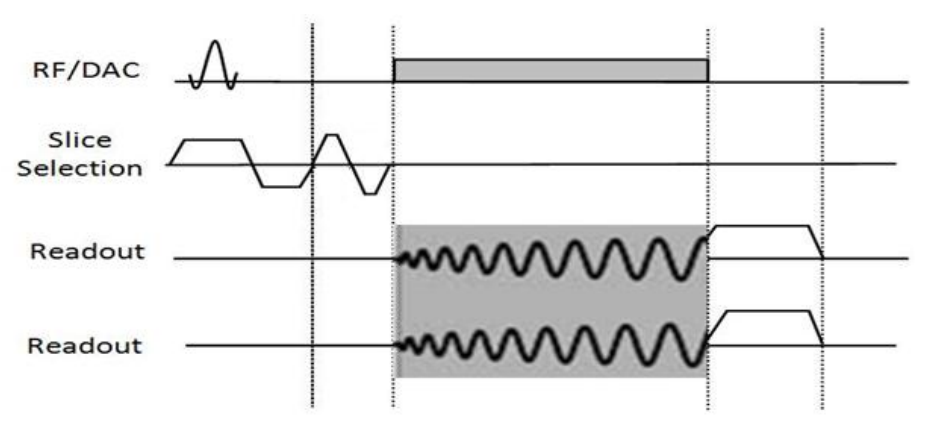

(a)

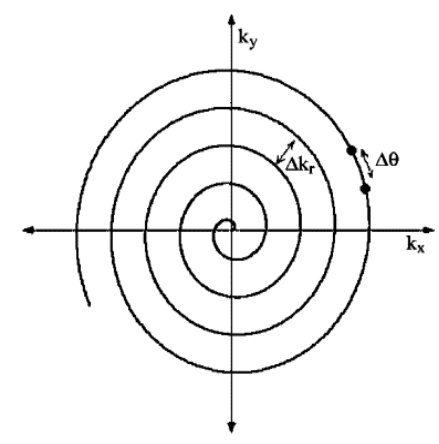

(b)

Figure 16: a schematic of spiral sequence with radial readout in two direction (a), and one interleave of spiral trajectory in k-space domain (b) [65].

\subsubsection{Cartesian trajectory}

In a conventional spin-warp sequence, $\mathrm{k}$-space data is acquired on a rectilinear grid. In this grid, any line of sampled data for each $G_{y}$ is placed parallel to $K_{x}$ axis. This type of $k$ space is called Cartesian trajectory which creates a spatial frequency array which can be presented directly to Fourier transform. Figure 15 depicts a 2-D gradient echo sequence producing Cartesian readouts. A slice select gradient $\left(\mathrm{G}_{\text {slice }}\right)$ is used to select the slice of interest followed by a phase encoding gradient which determines which line of $\mathrm{k}_{\mathrm{y}}$ should be acquired. Subsequently a frequency encoding gradient is applied with its initial part determining the starting $\mathrm{k}_{\mathrm{x}}$ point and the remaining part of the gradient producing $\mathrm{k}$-space data parallel to the $\mathrm{k}_{\mathrm{y}}$ axis. Image reconstruction with Cartesian trajectory is fast and straightforward due to the uniform rectilinear array. In addition, data collection in the rectilinear manner is robust to system imperfections such as $B_{0}$ inhomogeneity. However, this technique prolongs the scan time and usually TE and TR are longer than with other non-Cartesian trajectories. 


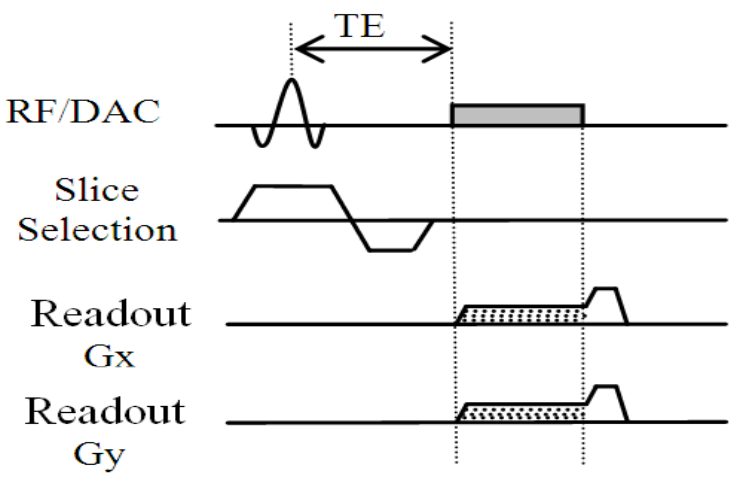

(a)

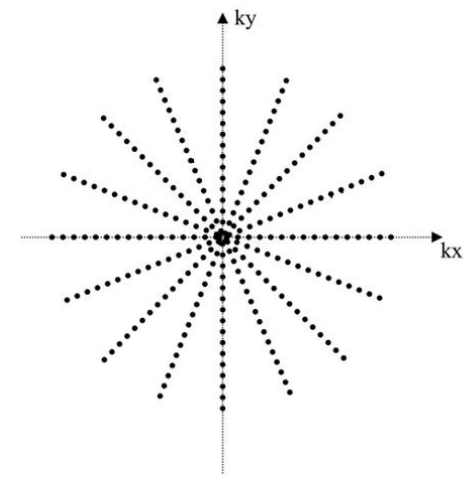

(b)

Figure 17: Radial pulse sequence with radial readout out in two directions (a), and radial trajectories in k-space domain showing oversampling of center of k-space in this method (b) [65].

\subsubsection{Spiral trajectory}

Spiral trajectory samples the k-space during a spiral traversal instead of rectilinear traversal (Figure 16). This trajectory is implemented by manipulating $G_{x}$ and $G_{y}$ gradients during continuous data sampling. Since the collected data points are not on a grid, an interpolation is performed on data points prior to Fourier transformation. Spiral trajectory can be very fast since all the data points can be acquired in one shot during one spiral with one excitation. This approach is referred to as single-shot spiral acquisition, however, it suffers from severe artifacts due to off-resonance. Multi-shot or interleaved spiral acquires multiple spiral shots in each excitation. As a result, the whole k-space is collected using multiple but shorter spirals. This leads to reduction of off-resonance artifact.

\subsubsection{Radial trajectory}

In radial scanning (also known as projection reconstruction [67]), data points in k-space are sampled along radius of a circle. The sampling starts from one endpoint of a diagonal and ends on the other end using two readout gradients simultaneously turned on in $\mathrm{x}$ and $\mathrm{y}$ 
directions. Figure 17 shows a radial pulse sequence and its k-space traversal. In this method, the data points in the center of $\mathrm{k}$-space are oversampled. This results in an improvement in signal to noise ratio (SNR) and the contrast but with the penalty of a lower spatial resolution. This acquisition is less sensitive to flow and motion compared to spin-warp (Cartesian trajectory) technique due to averaging in low spatial frequencies by oversampling of the center of k-space [68]. Since the trajectory lines need smaller phase encoding as with spiral acquisitions, gradients compared to Cartesian and spiral trajectories, TE is shorter with this technique. The disadvantage of this technique (as with spiral readout) is that the reconstruction is more complicated and slower due to non-linear sampling of k-space which makes the direct inverse Fourier transformation not possible, requiring preprocessing and data regridding.

\subsection{Ultra short TE (UTE) technique}

UTE technique was initially proposed for lung imaging [69-71] and improved the signal detection of tissues with $\mathrm{T}_{2}$ relaxation times as short as few hundred milliseconds [72-74].

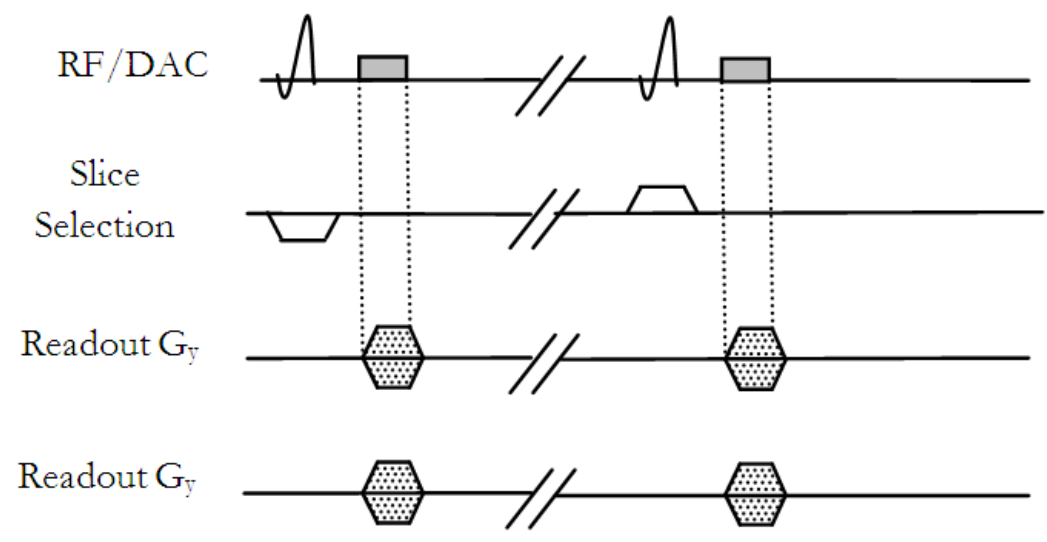

Figure 18: Conventional 2-D UTE sequence based on two back to back scan with half RF pulse [75]. 
This technique is based on employing half RF pulses and radial sampling starting from the

center of k-space. Figure 18 demonstrates the conventional UTE sequence based on two back to back scan with half RF pulse. In this sequence, a half RF pulse is applied with a slice excitation gradient without refocusing lobe to shorten the TE. The collected data is combined with the data acquired using the same sequence with inverted slice excitation gradient. Radial sampling is employed to collect the data starting from the center or k-space in contrast to regular radial sampling, where data sampling is started from one endpoint of kspace lines to the other endpoint on the diagonal. Compared to Cartesian trajectory, centerout radial sampling results in elimination of phase encoding gradient and rephasing gradient in the readout direction to get back to the center of k-space. This means sampling can be started immediately after ramp down of RF pulse and slice select gradient. In addition, data sampling can be performed during readout gradient ramp up (rising slope of gradient) using non-linear sampling [76] and therefore TE is defined as the distance between the center of RF pulse and the beginning of readout gradient which can be as short as tens of microseconds.

The UTE technique involves a radial sampling of Free Induction delay (FID), implying doubling of the number of radial profiles $\left(0-360^{\circ}\right)$ in comparison to the standard radial trajectory $\left(0-180^{\circ}\right)$. Since the reversed gradients are not used to form an echo and half RF pulses are not refocused, UTE is not spin echo or recalled gradient echo. But, since there is no echo, UTE technique is usually known as gradient echo.

In the conventional pulse sequences, Echo time (TE) is defined as the distance between the centers of RF pulse and readout gradient. This time is used to apply several gradients in pulse sequence such as slice select gradient, phase encoding gradient, the initial lobe of 
frequency encoding gradient which is the first half of readout gradient to reach the center of $\mathrm{k}$-space (rephasing lobe). These gradients cause the TE in conventional sequences to be on the order of few milliseconds. In this sequence, data is sampled based on rectilinear sampling using Cartesian trajectory as is shown in Figure 15.

\subsubsection{3-D Ultra-short TE}

As previously explained, conventional UTE is based on 2-D radial sampling of the FID. This technique suffers from partial volume effects as well as difficulties in the slice selection. Alternatively, UTE technique can be implemented using a 3-D technique to improve partial volume effects due to higher spatial resolution [78]. In addition, 3-D UTE does not have the difficulties of 2D UTE in slice selection which requires two half RF pulse with inverted slice select gradient. The radial readout trajectory in 3-D UTE is based on radial traversal of evenly spaced k-space lines starting from center of k-space and ending on the surface of a sphere with radius $\mathrm{K}_{\max }$ determined by spatial resolution in three spatial directions (Figure 19). Readout directions are defined by the angle $\varphi$ from $\mathrm{k}_{\mathrm{y}}$ axis and by angle of $\theta$ from $\mathrm{k}_{\mathrm{z}}$

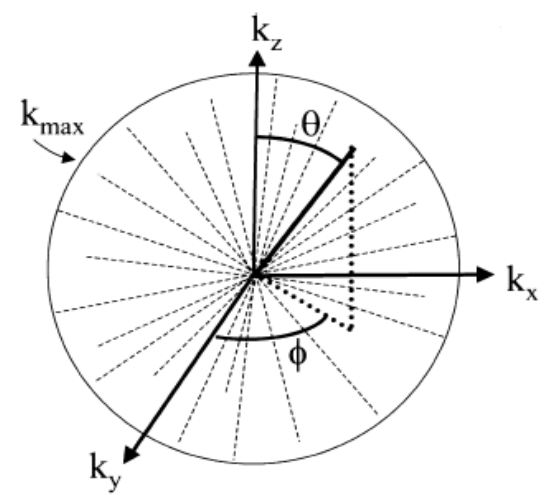

Figure 19: The radial readout trajectory in 3-D UTE. $K_{\max }$ is the radius of radial k-space which is determined by spatial resolution in three spatial directions [77]. 


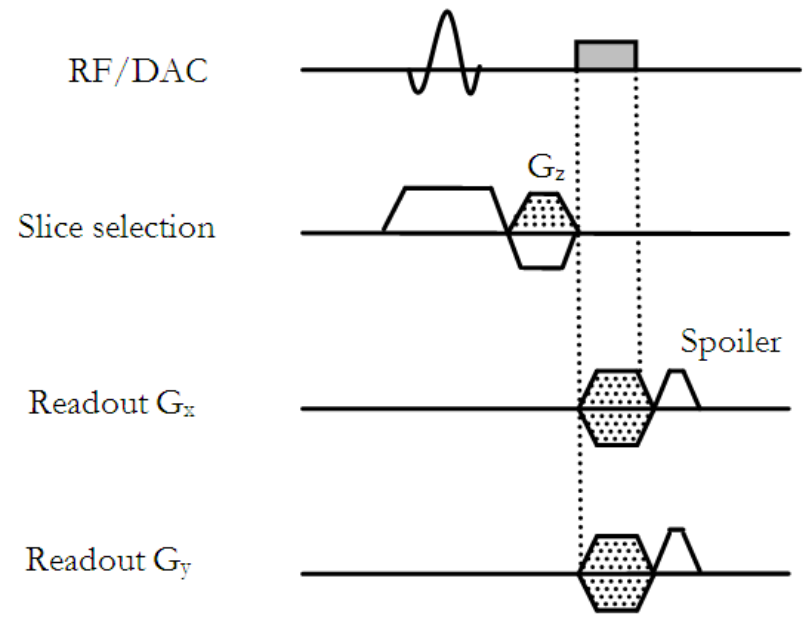

Figure 20: 3-D UTE sequence based on slice selective RF pulse using radial readout gradient in two directions.

axis. This technique can be performed using slice selective RF pulse $[79,80]$ as well as nonselective hard RF pulse. 3-D slice selective UTE is shown in Figure 20.

The slice selective 3-D UTE technique has not been practically used due to longer TE on the order of 1 millisecond. To achieve even shorter TE, a non-selective RF pulse can be used which results in slice rephasing gradient to be removed. A non-selective 3-D UTE using a hard RF pulse is shown in Figure 21 where TE is defined as the distance between the end of the RF pulse and the beginning of the readout gradient. The TE is only limited to transmit/receive switching time of the coil which can be reduced to as short as 8 microseconds [81]. 


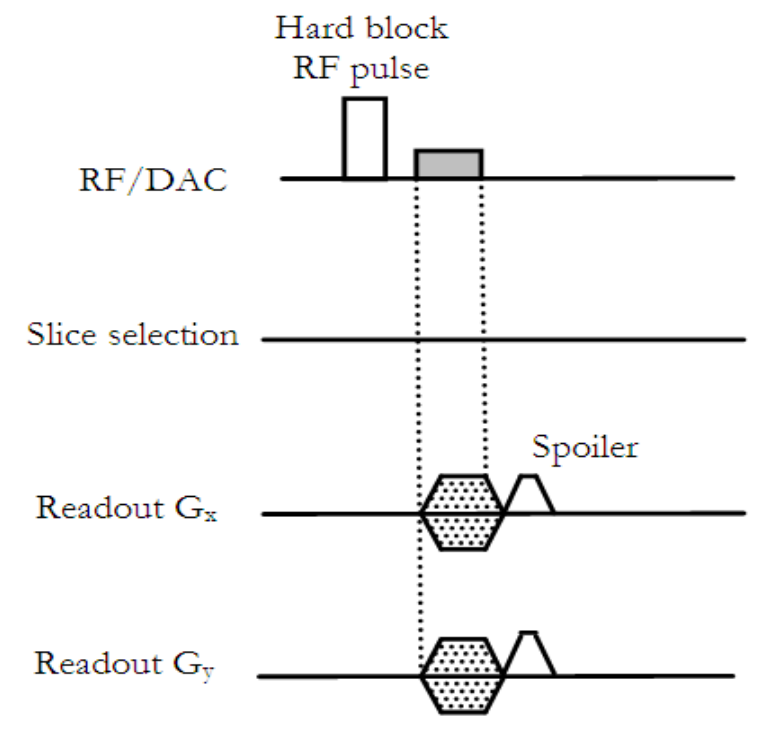

Figure 21: 3-D UTE sequence based on non-selective RF pulse using radial readout gradient in two directions. TE can be reduced to 8 microseconds using this sequence [82].

\subsubsection{Kooshball and stack-of-star trajectories}

3-D UTE imaging can be performed using angularly isotropic or non-isotropic sampling. Isotropic sampling, known as "kooshball" [83], yields isotropic spatial resolution. The kooshball trajectory includes multiple interleaves each a rotated version of the first one around $\mathrm{z}$ axis. Each interleave consists of multiple radial projection whose endpoints follow a spiral path running from one pole to the other on the surface of a sphere. Figure 22(a) illustrates one interleave of kooshball acquisition with endpoints of center-out gradients located on the surface of sphere. This technique has better flow and motion insensitivity as well as higher scan efficiency compared to Cartesian trajectory.

Figure 22(b) illustrates the non-isotropic stack-of-stars trajectory [84] where a cylindrical volume is acquired by sampling multiple slices using the center-out radial trajectory. A conventional phase encoding in $\mathrm{Z}$ direction is used to collect multiple slices in the through 


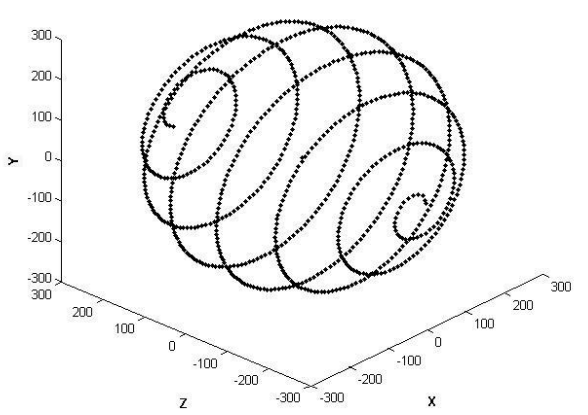

(a)

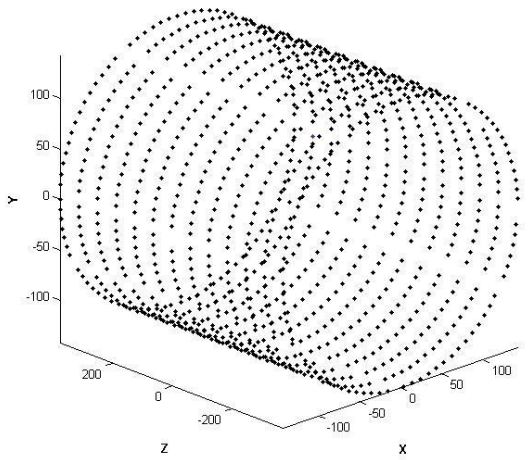

(b)

Figure 22: Two 3-D trajectory acquisition. One interleave of kooshball trajectory (a) and 13 slices of stackof-stars (b) is shown. The dot points are the end-point of projections in k-space.

plane direction. Unlike kooshball acquisition, this acquisition strategy is non-isotropic and hence the number of slices are adjustable leading to a shorter acquisition time. Having center-out k-space radial trajectories for both of these 3-D trajectory acquisitions helps reduce the effect of intravoxel dephasing and related phase artifacts due to inherent minimization of first moment of readout gradient by oversampling of the center of k-space $[2]$. 


\section{FLOW IMAGING AND MAGNETIC RESONANCE}

\section{ANGIOGRAPHY (MRA)}

\subsection{Introduction}

In the past few decades, many imaging techniques have been developed for blood flow imaging and quantification. X-ray radiography, X-ray Computer Tomography (CT) scan, nuclear medicine, and ultrasound have been used for flow imaging [85-88]. Each of these techniques has both advantages and disadvantages when compared to MRI. For instance, radiographic techniques and nuclear medicine suffers from ionizing radiation and contrast injection which make these techniques invasive, but at the same time they enjoy higher spatial resolution (radiography) and are more sensitive (nuclear medicine). Ultrasound flow imaging, on the other hand, is not accurate in case of air or bone appearance [89], yet is less costly than MRI. MRI, as an alternative technique, has been widely used for quantitative flow imaging [90-94]. The advantage of MRI in the context is that it can determine all three components of flow velocities. No other method has this capability.

Magnetic resonance angiography is one of the main techniques for flow visualization which is based on measuring an attribute of the flowing blood in the cardiovascular system. In the past, angiography was limited to x-ray angiography, wherein blood vessels are imaged after injection of a contrast agent to increase the contrast between blood and surrounding stationary tissues. However, this technique is invasive due to radiation exposure and may cause infection and bleeding. With MRI, however, in addition to contrast-enhanced MR 
angiography [95-99] which is available, non-contrast [100-105] methods are also possible. Non-contrast MRA does not require insertion of catheters and injection of contrast agent. In the case of non-contrast MRA, image acquisition is based either on inflow or phase effects in order to enhance signal from the flowing blood and suppressing the stationary tissue. Noncontrast MRA technique can be categorized into two major classes: 1- inflow MRI or timeof-flight (TOF) [106], 2- Phase Contrast Angiography (PCA) [107].

\subsection{Time-of-Flight (TOF)}

TOF is the most time-efficient MRA technique and is based on movement of enhanced unsaturated flowing blood into a saturated stationary tissue slice. TOF can be performed in two ways: black blood or bright blood imaging.

Black blood imaging is based on a spin echo acquisition. This technique voids the signal from flowing image while increasing the signal from stationary tissues. In this technique the flowing blood only experiences one RF pulse in contrast to stationary tissue which is excited by both $90^{\circ}$ and $180^{\circ}$ pulses. This fact results in low intensity for flowing blood compared to static tissue. For high velocities, most of the spins exit the slice of interest before applying the $180^{\circ}$ pulse and therefore appear dark in the image. One advantage of black blood imaging is that since all blood is void, turbulent flow creates a void signal similar to regular blood flow.

Bright blood TOF is a gradient echo technique which is based on signal enhancement caused by moving of unsaturated blood into the slice of interest which contains saturated blood by applying two consecutive $90^{\circ} \mathrm{RF}$ pulse. Unsaturated fresh spins which are replaced with saturated spins in the slice of interest only experience second RF pulse and therefore by 


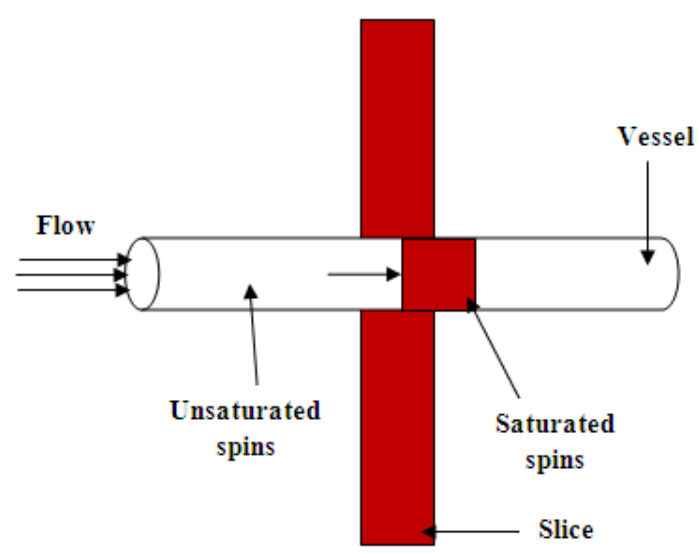

Figure 23: A schematic of blood refreshment TOF technique. Blood refreshment depends on flow velocity, volume thickness and repetition time TR.

detecting signal shortly after second RF pulse produce a higher signal intensity compared to stationary spins which experience both RF pulses. This technique has some significant

advantages. For instance, arteries or veins may be selectively imaged by the use of a presaturation protocol. In addition, in contrast to phase contrast angiography image subtraction is not necessary resulting in shorter scan time and computing requirements. Figure 23 shows a schematic of TOF technique where fresh blood moves into the slice that has experienced an RF pulse. In this case, the maximum contrast between flowing blood and stationary tissue is achieved when the blood is completely refreshed and the readout is immediately after excitation.

\subsection{Phase Contrast Angiography (PCA)}

Phase contrast angiography (PCA) is based the fact that flowing blood induces a phase shift in the MR signal which is proportional to velocity of flowing blood. There is a direct correlation between accumulated phase shift and signal intensity. In standard MR imaging, 


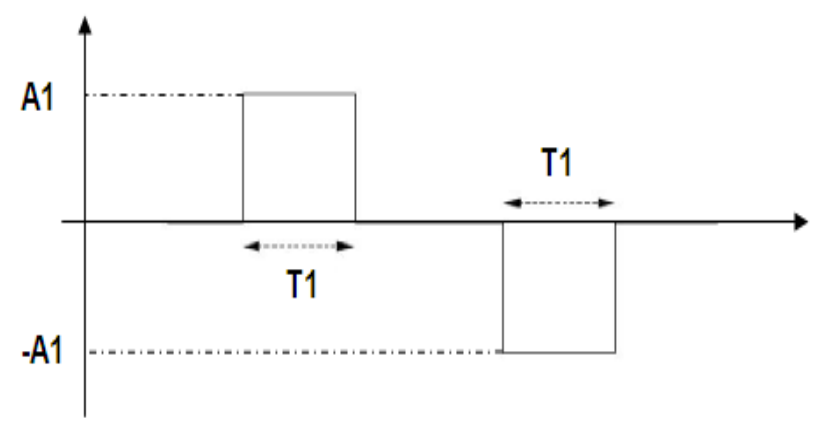

Figure 24: An example of flow sensitive gradient consisting of two lobes with identical shape.

signal intensity is the basis for image reconstruction and the acquired image is referred to as the magnitude image. However, in phase contrast MRI, the phase of spins in each voxel is the basis for image reconstruction and the reconstructed image is called the phase image. In

phase contrast a flow sensitive gradient is applied during acquisition and consequently phase of image correlates to velocity of flowing spins. The reconstructed image in this case is called phase velocity map or phase contrast image in which the phase image is mapped to describe the velocity difference between flowing spins across the image.

The flow sensitive gradient is a bipolar gradient consisting of two gradient pulses with identical shape and area but inverse amplitude (Figure 24). When a moving spin experience a bipolar gradient, the spin accumulates a net phase at the end of bipolar gradient. This accumulated phase is correlated to the velocity of spin during the flow sensitive gradient. However, static spins accumulate zero phase at the end of flow sensitive gradient because accumulated phase during opposite amplitude gradients cancel each other.

Phase changes, in addition to dependency on motion, are dependent on eddy currents and magnetic field inhomogeneity. Therefore, to remove the phase changes due to these issues, a flow compensated background image is acquired using flow compensated gradient 


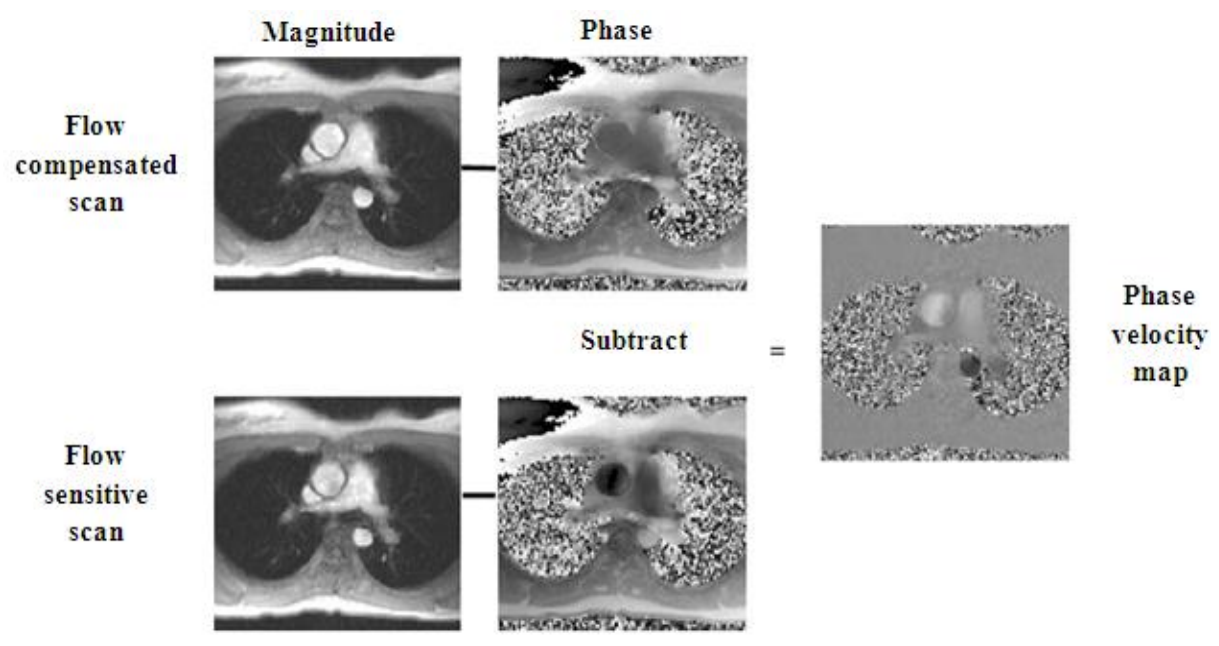

Figure 25: Magnitude and phase image for flow sensitive and compensated scan using phase contrast angiography and measured phase velocity map by subtraction of the phase of the two scans [2].

which is identical to flow sensitive gradient with opposite amplitudes. The phase contrast image with exclusive dependency on motion effects can be obtained by subtracting the flow compensated image from the flow sensitive image. Figure 25 demonstrates the magnitude

image and phase image acquired using a standards phase contrast sequence and phase velocity map is shown after subtraction of phase images in flow sensitive and flow compensated scans. In general, phase contrast imaging includes two, three or four acquisition to collect the flow sensitive images in different directions as well as one flow compensated image. The flow sensitive gradient can be applied in foot-to-head (FH), anterior-posterior (AP), and right-left (RL) directions. The flow compensated image can be subtracted from each of the flow sensitive images in these three directions and three PC images can be then constructed, each representing velocity in the corresponding direction.

\subsection{Flow quantification}


Based on physics of MR image formation, in the presence of a magnetic field gradient vector $G$, spins accumulate phase shift:

$$
\varphi=\gamma \int_{0}^{\mathrm{t}} \mathrm{G}\left(\mathrm{t}^{\prime}\right) \cdot r \mathrm{dt}^{\prime}
$$

where $\mathrm{G}\left(\mathrm{t}^{\prime}\right)$ is the time-varying magnetic field gradient and $\mathrm{r}$ is the position of spins. The position of spins can be written as

$$
r(t)=r_{0}+v t
$$

where motion terms higher than order one are assumed zero. $r_{0}$ is the initial position of the spin and $\mathrm{v}$ describes the velocity of the moving spin. By substituting (11) in (10) accumulated phase shift can be expressed as

$$
\varphi=\gamma \int_{0}^{\mathrm{t}} \mathrm{G}\left(\mathrm{t}^{\prime}\right) \cdot\left\{\mathrm{r}_{0}+\mathrm{vt}\right\} \mathrm{dt}^{\prime}=\gamma \mathrm{r}_{0} \cdot \mathrm{M}_{0}+\gamma \mathrm{v} \cdot \mathrm{M}_{1}
$$

where $\mathrm{M}_{0}$ and $\mathrm{M}_{1}$ are the zeroth and first moment of gradient which are defined as

$$
\begin{aligned}
& \mathrm{M}_{0}=\gamma \int_{0}^{\mathrm{t}} \mathrm{G}\left(\mathrm{t}^{\prime}\right) \mathrm{dt}^{\prime} \\
& \mathrm{M}_{1}=\gamma \int_{0}^{\mathrm{t}} \mathrm{t} \cdot \mathrm{G}\left(\mathrm{t}^{\prime}\right) \mathrm{dt^{ \prime }}
\end{aligned}
$$

To control the motion sensitivity of MR images, flow sensitive gradient can be applied during data acquisition (Figure 24). The first moment of this bipolar gradient, $\mathrm{M}_{1}$, can be adjusted according to the motion sensitivity and other gradients are conFigured to be insensitive to the motion and produce zero first moment of gradient. By designing a flow encoding gradient such that $\mathrm{M}_{0}$ is zero, the accumulated phase shift in (15) can be written as

$$
\varphi=\gamma \mathrm{v} \cdot \mathrm{M}_{1}
$$

Based on (18), the velocity of moving spins is proportional to phase shift as follows 


$$
\mathrm{V}=\frac{\phi}{\gamma \cdot \mathrm{M}_{1}}
$$

The velocity of spins can be measured in three directions via application of flow sensitive gradients in different directions. This can be performed by applying bipolar gradients in slice selection, phase encoding and frequency encoding directions between the slice select and readout gradients.

Since the phase value is only unique between $-\pi$ and $\pi$, a velocity value higher than a specific value cannot be encoded. The highest velocity encoded value which corresponds to a $\pi$ phase shift is known as velocity encoding or Venc and can be expressed as

$$
\text { Venc }=\frac{\pi}{\gamma \cdot \mathrm{M}_{1}}
$$

It is critically important to adjust the Venc value correctly because if the actual value of velocity is higher than Venc, the measured phase will be out of range of $[-\pi, \pi]$ and the measured velocity value in that case aliases back to a low value, causing artifacts. 


\section{DISTURBED AND TURBULENT FLOW QUANTIFICATION}

\subsection{Conventional techniques}

PC MRI, which was described in Chapter 4, is a reliable non-invasive technique for characterization and quantification of cardiovascular blood flow and has been employed for blood flow quantification in the heart [108, 109], aorta and aortic valve [110, 111], Carotid vessels $[112,113]$, and various cardiovascular diseases. However, this technique is not accurate in cases of atherosclerotic disease or where blood flow is disturbed or turbulent. Disturbed flow may be seen at bifurcations, branch point, and other regions of the arterial tree where blood flow is altered. These areas are more prone to atherosclerosis and narrowing in vessels causing turbulent flow and a high velocity jet distal to a stenosis $[5,47$, 114, 115]. Carotid bifurcation is one of the main sites of atherosclerosis and is a good example of complex and disturbed blood flow due to atypical geometry of this branch site [116].

A signal loss may appear distal to a stenosis due to this turbulent blood flow $[8,117]$. In addition, turbulent flow results in velocity fluctuations, leading to intravoxel dephasing and significant error in velocity measurement and assessment of blood flow. Intravoxel dephasing is produced due to distribution of various velocities in a voxel which leads to signal phase interference [118]. Various factors have been reported as the cause of signal loss in previous studies. Acceleration, higher order flow, and fluid mixing leading to intravoxel 
dephasing $[6,7]$, and phase mis-registration errors due to phase shift between phase encoding steps $[4,7]$ are main reasons for artifacts.

Several approaches have been developed to potentially reduce the signal loss and image artifacts in PC MR images [4-7]. Contribution of higher order motion such as acceleration[68, 10], partial volume effect and the voxel size [5, 119-122], accurate Velocity encoding parameter (Venc) [4], and aligning orientation of slice of interest with the jet flow [123, 124] (in this case flow encoding is not through-plane but in-plane) are some approaches used for reducing the signal loss due to intravoxel dephasing.

One important approach that results in significant impact in correction of the signal loss is reduction of the echo time (TE). Reducing the TE decreases the intravoxel dephasing and results in a higher signal to noise ratio and more reliable estimation of velocity in PC MRI. A shorter TE reduces the effect of intravoxel dephasing since velocity measurement times are shorter. Several groups have studied the effect of TE on signal loss and velocity quantification $[5,8-11,121,125,126]$. In [125], it was shown that the area of signal loss is increased by decreasing the pixel size and increased TE. Another study reported that by shortening the TE to $3.1 \mathrm{msec}$ in MRA of Carotid arteries, the signal loss reduced [11]. However, due to higher order of motion and velocity fluctuation even with TE=3.1 msec, a significant dephasing and signal loss appeared. In [10], the effect of TE and other parameters on signal loss was studied, and by varying the TE between 2.7 and $19 \mathrm{msec}$, it was demonstrated that the smallest signal loss was achieved for $\mathrm{TE}=2.7 \mathrm{msec}$. Another study reported that a spiral phase-contrast sequence with $\mathrm{TE}=1.6 \mathrm{msec}$ was capable of measuring flow in the case of high flow jet distal to stenosis [5]. Recently, a new PC sequence based on the ultra-short TE (UTE) technique was presented with TE reduced to $0.65 \mathrm{msec}$ [12]. 


\subsection{Ultra short TE (UTE) technique}

UTE technique was initially proposed for lung imaging [69-71] and recently has been employed for recognition of lung diseases such as cystic fibrosis and emphysema $[127,128]$. The ultra-short TE in this technique improves the signal detection of tissues with $\mathrm{T}_{2}$ relaxation time as short as few hundred milliseconds [72-74]. This technique has been used for several clinical applications such as cortical bone [129-131], Tendons and ligaments [132, 133], Atherosclerotic Plaque [134-136], knee [129, 137-139], Articular cartilage [140-144], spine [145], temporomandibular joint (TMJ) disk [146], liver [147, 148] and neuroimaging in

brain and central nervous system [149, 150]. Recently, a 2-D phase-contrast MR sequence was presented based on the ultra-short TE (UTE) technique with TE reduced to $0.65 \mathrm{msec}$ [12]. This study proved to be a more reliable technique for measuring high velocities due to robustness to intravoxel dephasing and signal loss. However, this technique had some disadvantages including underestimation of low flow rates.

\subsection{Shortcomings of Previous UTE-PC MRI Technique}

The first UTE-PC technique was presented by O'Brien et al. [9] for quantitative assessment of blood flow in stenotic valvular disease. The proposed 2-D UTE technique shortened the TE through two approaches: (i) by combining the slice select gradient with flow encoding/compensation gradient and (ii) by starting data acquisition from the beginning of gradient ramp using non-linear sampling. TE is defined in this case as the distance between the center of the RF pulse to the beginning of the readout gradient. The strategy results in few potential problems. The approach involves combination of an inverted slice select gradient with the flow encoding gradient in order to achieve a short TE. 
The slice select gradient was inverted to permit shorter total gradient duration. However, inverting the sign of slice select gradient causes more sensitivity to scanner imperfections, and in particular, $\mathrm{B}_{0}$ inhomogeneities, resulting in phase errors and velocity miscalculations. In general, $B_{0}$ inhomogeneity can cause a frequency offset $\left(\Delta \mathrm{w}=\gamma \Delta \mathrm{B}_{0}\right)$, leading to an offset in slice position $\left(\Delta \mathrm{z}=\Delta \mathrm{w} / \gamma \mathrm{G}_{\mathrm{s}}\right)$. This offset is more problematic for 2-D PC-UTE technique, since the slice select gradient is inverted for flow compensation scan compared to flow encoding scan and therefore slice position offset is double $\left(\Delta \mathrm{z}=2 \Delta \mathrm{w} / \gamma \mathrm{G}_{\mathrm{s}}\right)$. As previously shown in Equation (8), slice thickness depends on the amplitude of magnetic field gradient and therefore by decreasing the gradient strength to provide a high Venc, slice thickness increases and thereby slice position offset increases.

In addition, combining the flow encoding and slice select gradients leads to dependency of the slice select gradient on the velocity encoding gradient. This dependency causes limitations on the minimum achievable slice thickness and therefore the velocity-encoded gradient strength which is controlled by the velocity encoding (Venc) parameter. The minimum achievable TE with the technique reported in [12] was $0.65 \mathrm{msec}$ with Venc $=500$ $\mathrm{cm} / \mathrm{s}$ and a minimum slice thickness of $8.75 \mathrm{~mm}$ which often is not clinically applicable. Additionally, with this dependency, flow assessment is only possible in the through-plane direction

Another problem with the 2-D UTE-PC sequence is that, this technique underestimates the flow measurement in low flow rates compared to conventional technique. This is most likely due to large gradient strength required for low Venc in order to shorten the TE. This large gradient strength can cause eddy current error and background phase offset. At a flow 


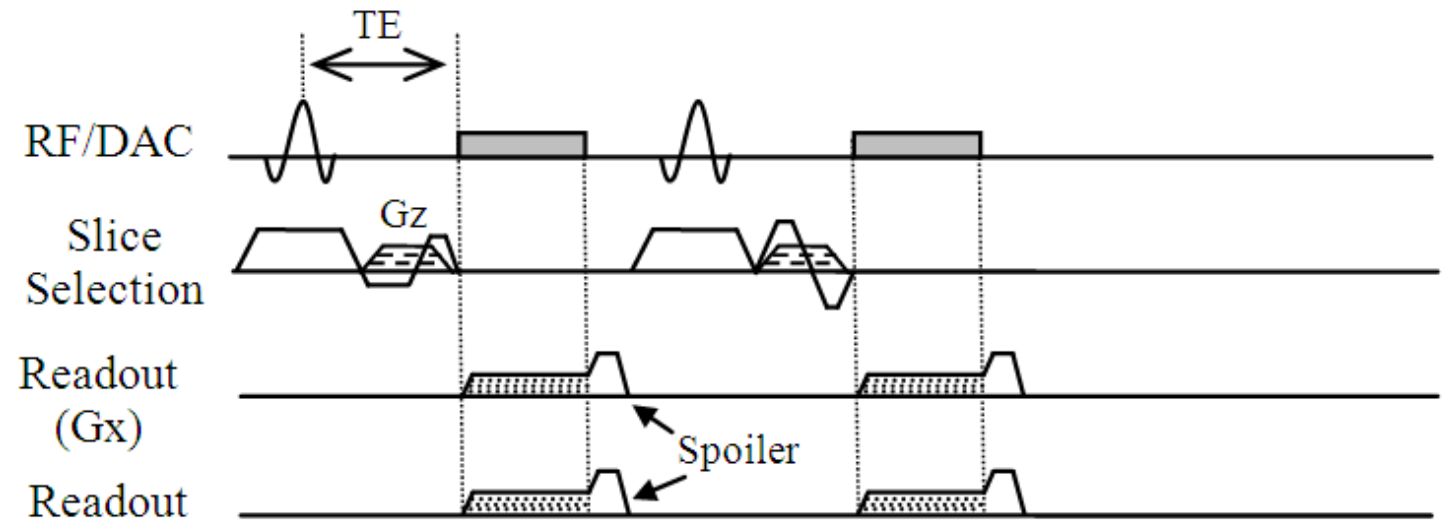

(Gy)

Flow encoding scan Flow compensated scan

Figure 26: The proposed 3-D UTE-PC MRI sequence. The flow sensitive (encoding) and compensated scans are acquired through combination of a bipolar gradient and refocusing part of slice select gradient.

Gradient $G_{z}$ is applied to acquire multiple slices in a volume using stack of star strategy.

rate such as $100 \mathrm{~mL} / \mathrm{s}$ (average velocity of $44 \mathrm{~cm} / \mathrm{sec}$ ) the error exceed $25 \%$ using this technique [9].

As a part of this dissertation, a novel 3-D cine UTE-PC sequence has been developed to obviate the problems with the previous UTE-PC technique. In addition, it was shown that the correction of phase errors produced by gradient delays improves the result of the UTEPC technique. The proposed sequence has been utilized to quantitatively measure blood velocity in an $87 \%$ area stenosis phantom under steady flow as well as in the carotid bifurcation which is associated with disturbed blood flow in normal volunteers.

\subsection{3-D UTE Phase-Contrast (PC) MRI}

Figure 26 shows the proposed 3-D UTE-PC pulse sequence where the slice excitation gradient is combined with flow encoding/compensation gradient. As with the previous 2-D technique, k-space sampling starts with the rising slope of readout gradient. Two back-to- 
back RF pulse and readout gradients are used in order to construct one flow sensitive image and one flow compensated (reference) image. In comparison to [12], the sign of slice

excitation gradient is not inverted due to disadvantages of inverting slice excitation. The inversion of slice select gradient lessens the gradient lobes to be played out during TE and hence leads to shorter TE. However, this gradient inversion results in the high sensitivity of the sequence to hardware imperfection and the consequent phase errors. In order to reduce the gradient duration during TE and in order to reduce the higher sensitivity of conventional 2-D UTE to hardware imperfections in the proposed technique, only the slice refocusing Section of the slice select gradient is combined with the flow encoding gradient. This, results in slightly longer TE compared to 2-D UTE-PC but short enough to resolve the introvoxel dephasing. In addition, the dependency of flow encoding and slice thickness is reduced compared to previous techniques and slice thickness is significantly decreased. The minimum achievable slice thickness for Venc $=500$ using 2-D UTE-PC technique is $8.75 \mathrm{~mm}$ whereas in the proposed sequence slice thickness can be adjusted as necessary.

The readout trajectory in UTE is based on radial traversal of evenly spaced k-space lines starting from the center of $\mathrm{k}$-space. Having center-out k-space radial trajectory reduces the effect of intravoxel dephasing due to inherent minimization of first moment of readout gradient by oversampling of the center of $\mathrm{k}$-space [2]. This oversampling in the center of kspace results in higher SNR for lower spatial frequencies compared to higher spatial frequencies and therefore decreases the motion artifact and pulsatile flow induced artifacts.

In the proposed technique, 3-D non-Cartesian trajectory acquisition based on the "stackof-stars" [84] strategy was employed to collect multiple slices in a cylindrical volume through the flow encoding direction. Stack-of-star trajectory was explained in Section 3.5.2. A phase 
encoding gradient in $Z$ direction, $G_{z}$, is employed to collect multiple slices along throughplane flow direction.

\subsection{Phase error correction}

Center-out k-space radial trajectories help to reduce the effect of intravoxel dephasing and related phase artifacts due to inherent first moment minimization of the readout gradient by oversampling the center of k-space [2]. However, center-out k-space lines are sensitive to phase errors due to several parameters such as gradient delays, eddy currents, and $\mathrm{B}_{0}$ field inhomogeneity. This can result in a mis-centering of k-space trajectory and leads to image artifact as well as erroneous phase calculations. To resolve the phase error in the proposed UTE technique with center-out radial readout, a phase error correction technique has been developed based on auto-correlation [151]. With this phase correction, the first order term of the phase along the readout direction due to gradient delays is eliminated.

Since the k-space in radial trajectory is a non-uniformly sampled, an efficient algorithm for reconstructing the image is necessary. This algorithm is known as Non-Uniform Fast Fourier Transform (NFFT) which includes several steps to produce the image from acquired k-space [152]. Using this technique and regridding of non-uniform k-space and rectilinear shape k-space is reproduced to facilitate image reconstruction. For more descriptions about NFFT algorithm, see [152].

Since the gradient delays in three physical gradient directions are independent, the consequent k-space mis-centering and phase errors in each three directions are independent as well. Assuming regridding of radial k-space to a rectilinear k-space, and three independent 
phase errors in three k-space directions, the phase distorted MR image $f_{1}(x, y, z)$ can be expressed as:

$$
f_{1}(x, y, z)=f(x, y, z) e^{i\left(x . \Delta \phi_{x}+y \cdot \Delta \phi_{y}+z . \Delta \phi_{z}\right)}
$$

where f, $\Delta \phi_{\mathrm{x}}, \Delta \phi_{\mathrm{y}}$, and $\Delta \phi_{\mathrm{z}}$ represent the original image matrix without phase error and phase error in $\mathrm{x}, \mathrm{y}$, and $\mathrm{z}$ directions in the measured image, respectively. Considering the reconstructed data as a $3-\mathrm{D}$ volume with $\mathrm{M} \times \mathrm{N} \times \mathrm{W}$ voxels, matrix $\mathrm{f}$ has the same number of elements.

By repeating the same scan with inverted readout gradients, the phase errors in three directions will be inverted. Thus, the reconstructed image from the second scan is

$$
\mathrm{f}_{2}(\mathrm{x}, \mathrm{y}, \mathrm{z})=\mathrm{f}(\mathrm{x}, \mathrm{y}, \mathrm{z}) \mathrm{e}^{-\mathrm{i}\left(\mathrm{x} \cdot \Delta \phi_{\mathrm{x}}+\mathrm{y} \cdot \Delta \phi_{\mathrm{y}}+\mathrm{z} \cdot \Delta \phi_{\mathrm{z}}\right)}
$$

Considering independent phase error in three directions, normalized cross-correlations between two scans can be performed separately in each direction using $\mathrm{M} \times \mathrm{N} \times \mathrm{W}$ matrices $\mathrm{f}_{1}$ and $\mathrm{f}_{2}$. To calculate the cross-correlation, matrix $\mathrm{C}_{\mathrm{z}}$ can be written as

$$
C_{z}=\sum_{m} f_{1}(x, y, m) f_{2}^{*}(x, y, m)
$$

Assuming phase error only in $z$ direction, if we substitute (21) and (22) in (23), we find

$$
C_{z}=\sum_{m} f(x, y, m) f^{*}(x, y, m) e^{-i 2\left(z . \Delta \phi_{z}\right)}
$$

where $\mathrm{C}_{\mathrm{z}}$ is the $\mathrm{M} \times \mathrm{N}$ correlation matrix between two scan in $\mathrm{z}$ direction. Normalized crosscorrelation between the two scans in $\mathrm{z}$ direction can be expressed as

$$
\mathrm{R}_{\mathrm{z}}=\frac{\mathrm{C}_{\mathrm{z}}}{\left|\mathrm{C}_{\mathrm{z}}\right|}
$$




$$
R_{z}=\frac{\sum_{m} f(x, y, m) f^{*}(x, y, m) e^{-i 2\left(z . \Delta \phi_{z}\right)}}{\left|\sum_{m} f(x, y, m) f^{*}(x, y, m)\right|}
$$

Since $f(x, y, z) f^{*}(x, y, z)$ is positive and real valued for MR images and its phase is always zero, phase shift in $z$ directions can be measured as

$$
\begin{gathered}
\arg \left(\mathrm{R}_{\mathrm{z}}\right)=\arg \left(\frac{\sum_{\mathrm{m}} \mathrm{f}(\mathrm{x}, \mathrm{y}, \mathrm{m}) \mathrm{f}^{*}(\mathrm{x}, \mathrm{y}, \mathrm{m}) \mathrm{e}^{-\mathrm{i} 2\left(\mathrm{z} \cdot \Delta \phi_{\mathrm{z}}\right)}}{\left|\sum_{\mathrm{m}} \mathrm{f}(\mathrm{x}, \mathrm{y}, \mathrm{m}) \mathrm{f}^{*}(\mathrm{x}, \mathrm{y}, \mathrm{m})\right|}\right) \\
\arg \left(\mathrm{R}_{\mathrm{z}}\right)=2 \mathrm{z} \cdot \Delta \phi_{\mathrm{z}} \\
\Delta \phi_{\mathrm{z}}=\frac{1}{2 \mathrm{z}} \cdot \arg \left(\mathrm{R}_{\mathrm{z}}\right)
\end{gathered}
$$

From Equation (27), it can be seen that the phase shift in $z$ direction is proportional to the phase of normalized cross-correlation of two scans. Alternately, phase shift in $\mathrm{x}$ and $\mathrm{y}$ directions, $\Delta \phi_{\mathrm{x}}$ and $\Delta \phi_{\mathrm{y}}$, can be calculated using

$$
\begin{aligned}
& \Delta \phi_{\mathrm{x}}=\frac{1}{2 \mathrm{x}} \cdot \arg \left(\mathrm{R}_{\mathrm{x}}\right) \\
& \Delta \phi_{\mathrm{y}}=\frac{1}{2 \mathrm{y}} \cdot \arg \left(\mathrm{R}_{\mathrm{y}}\right)
\end{aligned}
$$

where $R_{x}$ and $R_{y}$ are

$$
\begin{aligned}
& \mathrm{R}_{\mathrm{x}}=\frac{\sum_{\mathrm{k}} \mathrm{f}(\mathrm{k}, \mathrm{y}, \mathrm{z}) \mathrm{f}^{*}(\mathrm{k}, \mathrm{y}, \mathrm{z}) \mathrm{e}^{-\mathrm{i} 2\left(\mathrm{x} \cdot \Delta \phi_{\mathrm{x}}\right)}}{\left|\sum_{\mathrm{k}} \mathrm{f}(\mathrm{k}, \mathrm{y}, \mathrm{z}) \mathrm{f}^{*}(\mathrm{k}, \mathrm{y}, \mathrm{z})\right|} \\
& \mathrm{R}_{\mathrm{y}}=\frac{\sum_{\mathrm{j}} \mathrm{f}(\mathrm{x}, \mathrm{j}, \mathrm{z}) \mathrm{f}^{*}(\mathrm{x}, \mathrm{j}, \mathrm{z}) \mathrm{e}^{-\mathrm{i} 2\left(\mathrm{y} \cdot \Delta \phi_{\mathrm{y}}\right)}}{\left|\sum_{\mathrm{j}} \mathrm{f}(\mathrm{x}, \mathrm{j}, \mathrm{z}) \mathrm{f}^{*}(\mathrm{x}, \mathrm{j}, \mathrm{z})\right|}
\end{aligned}
$$

By measuring the phase error in each direction the phase corrected image will be

$$
\mathrm{f}(\mathrm{x}, \mathrm{y}, \mathrm{z})=\mathrm{f}_{1}(\mathrm{x}, \mathrm{y}, \mathrm{z}) \mathrm{e}^{-\mathrm{i}\left(\mathrm{x} \cdot \Delta \phi_{\mathrm{x}}+\mathrm{y} \cdot \Delta \phi_{\mathrm{y}}+\mathrm{z} \cdot \Delta \phi_{\mathrm{z}}\right)}
$$

Based on the Fourier shift theorem, Equation (32) can be written as 

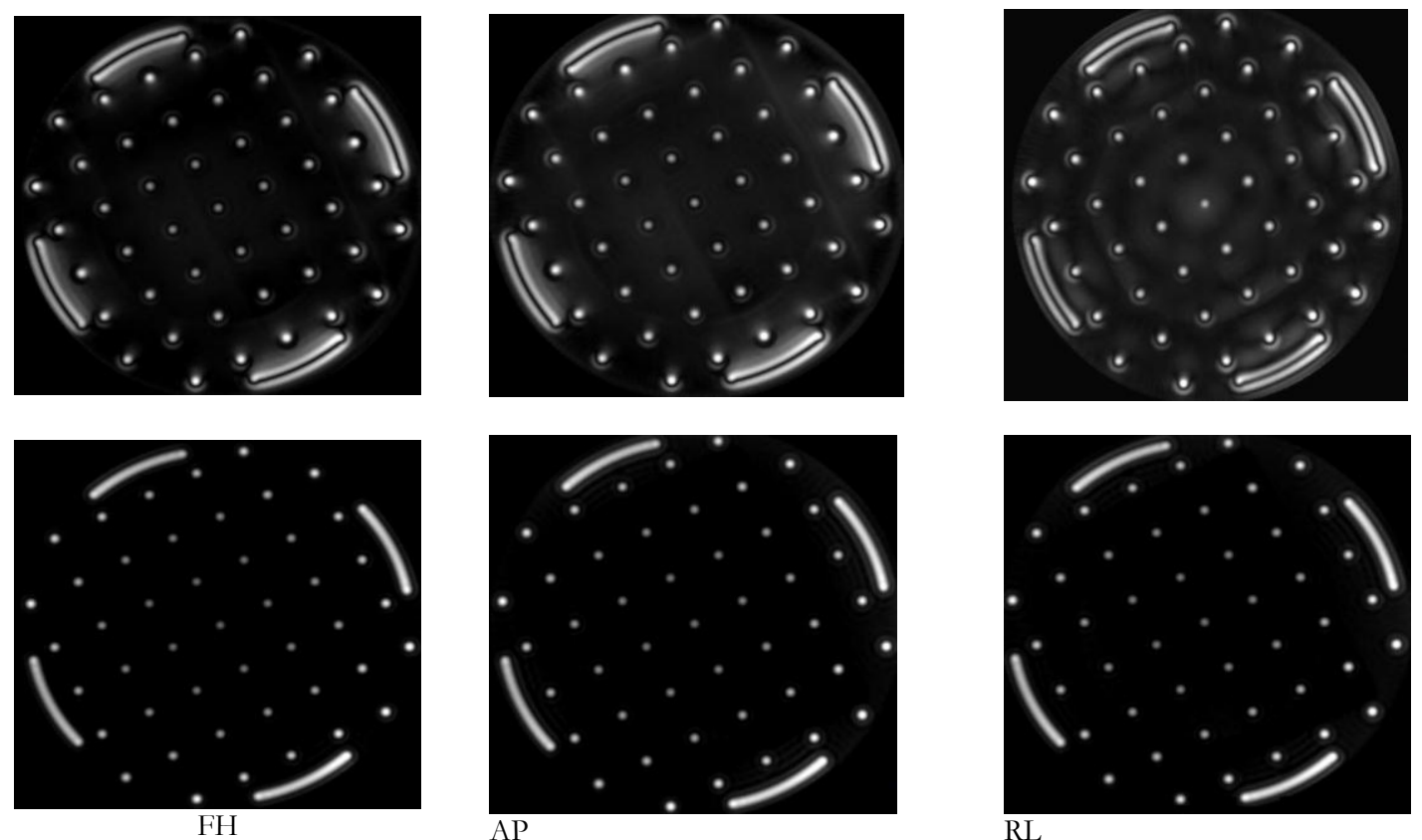

Figure 27: Phantom result for different flow directions. The first row shows the original images using 3-D UTE-PC imaging in FH, AP, and RL flow direction respectively. The second row shows the result after phase error correction.

$$
\mathcal{F}\left(\mathrm{k}_{\mathrm{x}}, \mathrm{k}_{\mathrm{y}}, \mathrm{k}_{\mathrm{z}}\right)=\mathcal{F}_{1}\left(\mathrm{k}_{\mathrm{x}}+\Delta \mathrm{k}_{\mathrm{x}}, \mathrm{k}_{\mathrm{y}}+\Delta \mathrm{k}_{\mathrm{y}}, \mathrm{k}_{\mathrm{z}}+\Delta \mathrm{k}_{\mathrm{z}}\right)
$$

where $\mathcal{F}$ and $\mathcal{F}_{1}$ are Fourier transform of images $\mathrm{f}_{1}$ and $\mathrm{f}_{2}$ in k-space domain. $\Delta \mathrm{k}_{\mathrm{x}}, \Delta \mathrm{k}_{\mathrm{y}}$, and $\Delta \mathrm{k}_{\mathrm{z}}$ are trajectory delays in $\mathrm{k}_{\mathrm{x}}, \mathrm{k}_{\mathrm{y}}$, and $\mathrm{k}_{\mathrm{z}}$ directions which are equal to $\Delta \phi_{\mathrm{x}} / 2 \pi, \Delta \phi_{\mathrm{y}} / 2 \pi$, and $\Delta \phi_{\mathrm{z}} / 2 \pi$ respectively.

\subsection{Phase error correction and calibration using Static phantom}

In order to reduce the phase error, a one-time calibration is performed by scanning a static phantom prior to the in-vivo scan based on the described phase correction technique.

Imaging of static phantom was performed on an Achieva 1.5T Philips scanner using a combined 16-element SENSE head coil with following parameters: TE/TR = 1.09/6.2 ms, $\mathrm{FOV}=180 \times 180 \mathrm{~mm}$, and spatial resolution $=1.17 \times 1.17 \times 5.0 \mathrm{~mm}$. These parameters were 
selected identical to the scan parameters for in-vivo studies. Alternatively, the scan parameters were selected identical to stenotic phantom scan for flow phantom study.

The trajectory delays for each k-space directions were measured using the proposed phase error correction technique. Due to implementation difficulty for application of different trajectory delay to different $\mathrm{k}$-space directions and also being aware of the fact that trajectory delays have quite similar values in all directions, the average trajectory delays in three directions is applied to all three directions in all subsequent studies. It should be noted that trajectory delays applied to flow phantom study and in-vivo study are slightly different due to different scan parameters in flow phantom and in-vivo scans.

Figure 27 demonstrates the results of static phantom study using proposed 3-D UTE-PC sequence with stack-of-stars trajectory in three flow directions. The top row shows the three images with different flow directions without trajectory delay correction. The bottom row shows the images after calculation of trajectory delay using proposed phase error correction method and their subsequent application for each direction. The images after trajectory delay

correction reveal significantly less phase error resembling ghosting in first row images. This phase error has considerable effect in phase-contrast technique and flow quantification wherein phase variation in flowing blood is desirable and any other source of phase deviation should be eliminated.

\subsection{Steady flow in stenotic flow phantom}

\subsubsection{Experimental setup}

An idealized rigid model of axisymmetric Gaussian shape was machined from transparent acrylic. We initially aimed the occlusion to have a $90 \%$ area narrowing at the throat. 


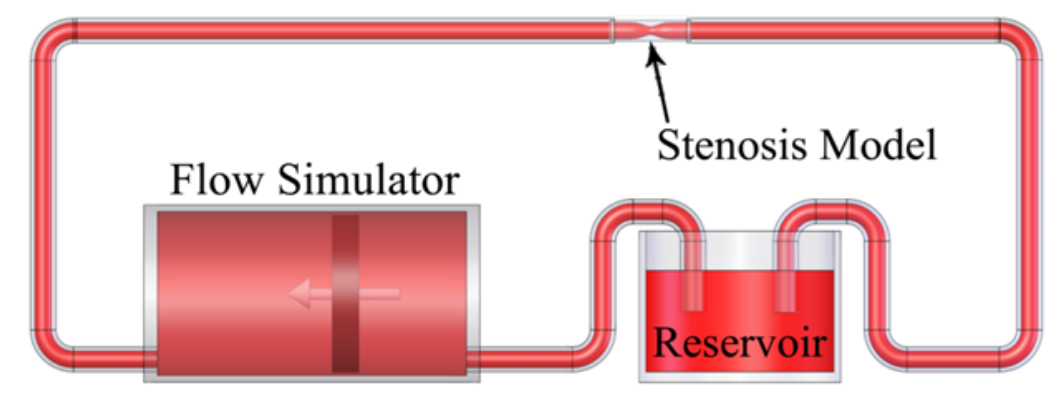

Figure 28: Schematic of the experimental setup showing the test Section with $87 \%$ area stenosis which was machined in-house from clear acrylic plastic.

However, later, the exact geometry was measured with high-resolution CT $(0.22 \times 0.22 \times$ $0.625 \mathrm{~mm}^{3}$ ) and the narrowing was determined to be $87 \%$. There were additional imperfections in fabrication of the phantom which caused the phantom geometry to not be entirely axi-symmetric. The stenosis diameter is $25.4 \mathrm{~mm}$ at the inlet and narrows down to $9.04 \mathrm{~mm}$ at the throat. To ensure fully developed laminar flow, devoid of disturbance, at the entrance of the model, a 75-cm long straight rigid acrylic tube was positioned upstream of the phantom. Fluid viscosity was 0.0043 Pa.s and fluid density was $1060 \mathrm{~kg} / \mathrm{m}^{3}$ at 65 degree F. Figure 28 shows the stenotic phantom setup in a closed loop flow system. A CardioFlow 1000 programmable pump (Shelley Medical Imaging Technologies, London, Ontario, Canada) was used for generating steady and pulsatile flows.

The viscosity of the blood-mimicking solution utilized in the MRI experiments was measured using a LVT Cone-Plate viscometer (Brookfield Labs., Stoughton, MA, USA) to be 0.022 Pa.s at $68^{\circ} \mathrm{F}$. The density of the solution was $1600 \mathrm{~kg} / \mathrm{m}^{3}$. The solution had a T1 and T2 value of $500 \mathrm{~ms}$ and $45 \mathrm{~ms}$, respectively, at a main field of 3T.

Three flow rates indicating low $(\mathrm{Q}=13.2 \mathrm{~mL} / \mathrm{s})$, medium $(\mathrm{Q}=39.4 \mathrm{~mL} / \mathrm{s})$, and high $(\mathrm{Q}=300 \mathrm{~mL} / \mathrm{s})$ flow rate were studied. These flow rates translate to the following Reynolds 
numbers at the inlet: $\operatorname{Re}=160, \operatorname{Re}=480, \operatorname{Re}=3618$, and the following Reynolds numbers at the throat: $\operatorname{Re}=440, \operatorname{Re}=1324, \operatorname{Re}=10022$, respectively.

\subsubsection{Imaging Protocol}

Imaging was performed on an Achieva 1.5T Philips scanner using a 16-element SENSE knee coil. The Imaging volume covered $\sim 50 \mathrm{~mm}$ of phantom and the scan was repeated 6 times to covers $300 \mathrm{~mm}$ of phantom including about $50 \mathrm{~mm}$ proximal and $250 \mathrm{~mm}$ distal to the throat of the stenosis (Figure 19). Each scan included 17 slices for Cartesian and spiral trajectories with the slice thickness of $4 \mathrm{~mm}$ and $1 \mathrm{~mm}$ overlap between slices and 10 slices for 3-D UTE sequence with the slice thickness of $4 \mathrm{~mm}$ and no overlap between slices. For the spiral trajectory, 30 interleaves each with $4 \mathrm{~ms}$ readouts were used. The other parameters for Cartesian, spiral, and 3-D UTE PC MRI are shown in Table 1.

Table 1: Acquisition parameters at $Q=13.2,39.4$, and $300 \mathrm{~mL} / \mathrm{s}$ for Cartesian, spiral, and 3-D UTE PC MRI for Phantom Studies. The Cartesian and the spiral acquisitions were multi-slice 2D acquisitions

\begin{tabular}{|c|c|c|c|}
\hline Parameter & Cartesian PC MRI & Spiral PC MRI & 3-D UTE-PC MRI \\
\hline FOV [mm] & $120 \times 120 \times 51$ & $120 \times 120 \times 51$ & $120 \times 120 \times 40$ \\
\hline $\mathrm{TE}$ in $\mathrm{Q}=13.2(\mathrm{~ms})$ & 5 & 3.5 & 2.5 \\
\hline$T R$ in $Q=13.2(\mathrm{~ms})$ & 7.9 & 9.9 & 12.7 \\
\hline $\mathrm{TE}$ in $\mathrm{Q}=39.4(\mathrm{~ms})$ & 4.4 & 2.9 & 1.9 \\
\hline$T R$ in $Q=39.4(\mathrm{~ms})$ & 7.3 & 9.3 & 8.6 \\
\hline $\mathrm{TE}$ in $\mathrm{Q}=300(\mathrm{~ms})$ & 4.2 & 2.5 & 0.95 \\
\hline$T R$ in $Q=300(\mathrm{~ms})$ & 7.3 & 8.8 & 5.7 \\
\hline $\begin{array}{l}\text { Venc at } Q=13.2,39.4, \\
300(\mathrm{~mL} / \mathrm{s})\end{array}$ & $30,75,500$ & $30,75,500$ & $30,75,500$ \\
\hline $\begin{array}{l}\text { Spatial resolution } \\
(\mathrm{mm})\end{array}$ & $1.5 \times 1.5 \times 4.0$ & $1.5 \times 1.5 \times 4.0$ & $1.5 \times 1.5 \times 4.0$ \\
\hline Flip angle (degree) & 12 & 12 & 12 \\
\hline $\begin{array}{c}\text { Imaging time } \\
\text { for one FOV (min) }\end{array}$ & 3: 07 & $1: 59$ & $2: 36$ \\
\hline
\end{tabular}




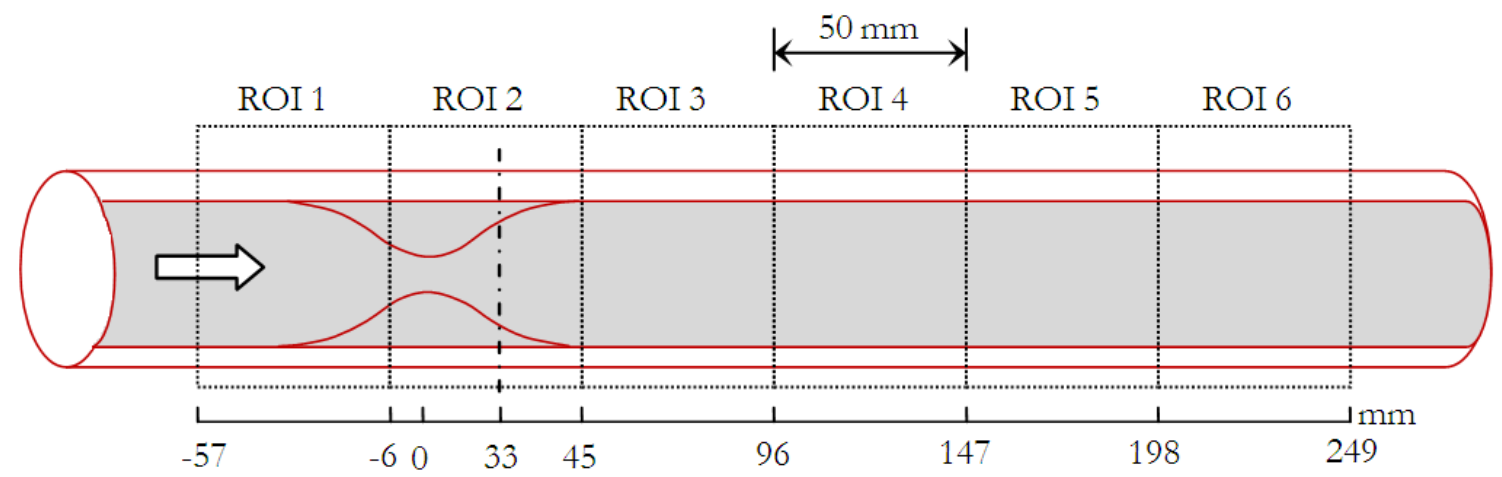

Figure 29: A schematic of the geometry of the phantom with six ROI where data were collected. The dash line shows the position of a slice $33 \mathrm{~mm}$ distal to stenosis.
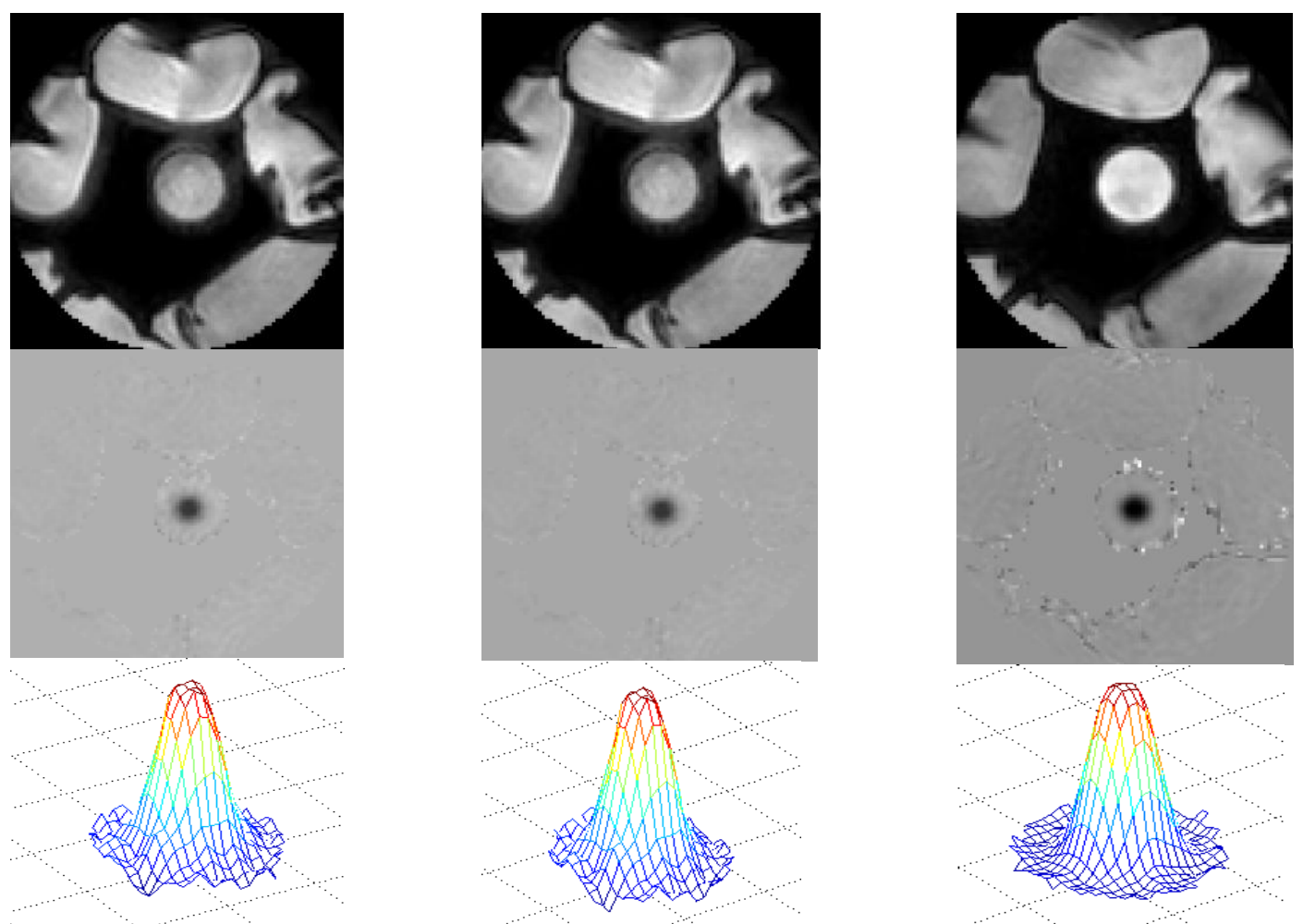

Figure 30: Magnitude image (first row), velocity image (second row), and velocity profile (third row) for low flow rate $\mathrm{Q}=13.2 \mathrm{~mL} / \mathrm{s}$ at $33 \mathrm{~mm}$ distal to throat of the stenosis using Cartesian (first column), spiral (second column), and 3-D UTE (third column) PC MRI sequences. The magnitudes, flows, and velocity profiles are in agreement for three techniques. 
Table 2: Average flow rate measured through the phantom (averaged over 85 slices - $25 \mathrm{~cm}$ of length along the phantom) $-5 \mathrm{~cm}$ proximal to throat to $20 \mathrm{~cm}$ distal to throat. Re number is measured at the throat of phantom.

\begin{tabular}{|c|c|c|c|c|c|c|}
\hline & 13.2 & $\begin{array}{l}\text { flow } \\
(\operatorname{Re}=440)\end{array}$ & $\begin{array}{r}1 \\
39.41\end{array}$ & $\begin{array}{l}\text { m flow } \\
(\operatorname{Re}=1324)\end{array}$ & $300 \mathrm{~m}$ & $\begin{array}{l}\text { flow } \\
\operatorname{Re}=10022)\end{array}$ \\
\hline & Flow & Error (\%) & Flow & Error (\%) & Flow & Error (\%) \\
\hline Cartesian & 13.3 & 1 & 39.29 & 0.3 & 235.09 & 21.6 \\
\hline Spiral & 12.61 & 4.5 & 36.55 & 7.2 & 254.62 & 15.1 \\
\hline 3-D UTE & 13.91 & 5.3 & 40.55 & 2.8 & 305.99 & 2 \\
\hline
\end{tabular}

\subsubsection{Results}

Figure 30 displays the magnitude image as well as the flow and velocity profiles for flow rate of $\mathrm{Q}=13.2 \mathrm{ml} / \mathrm{s} 33 \mathrm{~mm}$ distal to throat of the stenosis for Cartesian, spiral and 3-D UTE PC
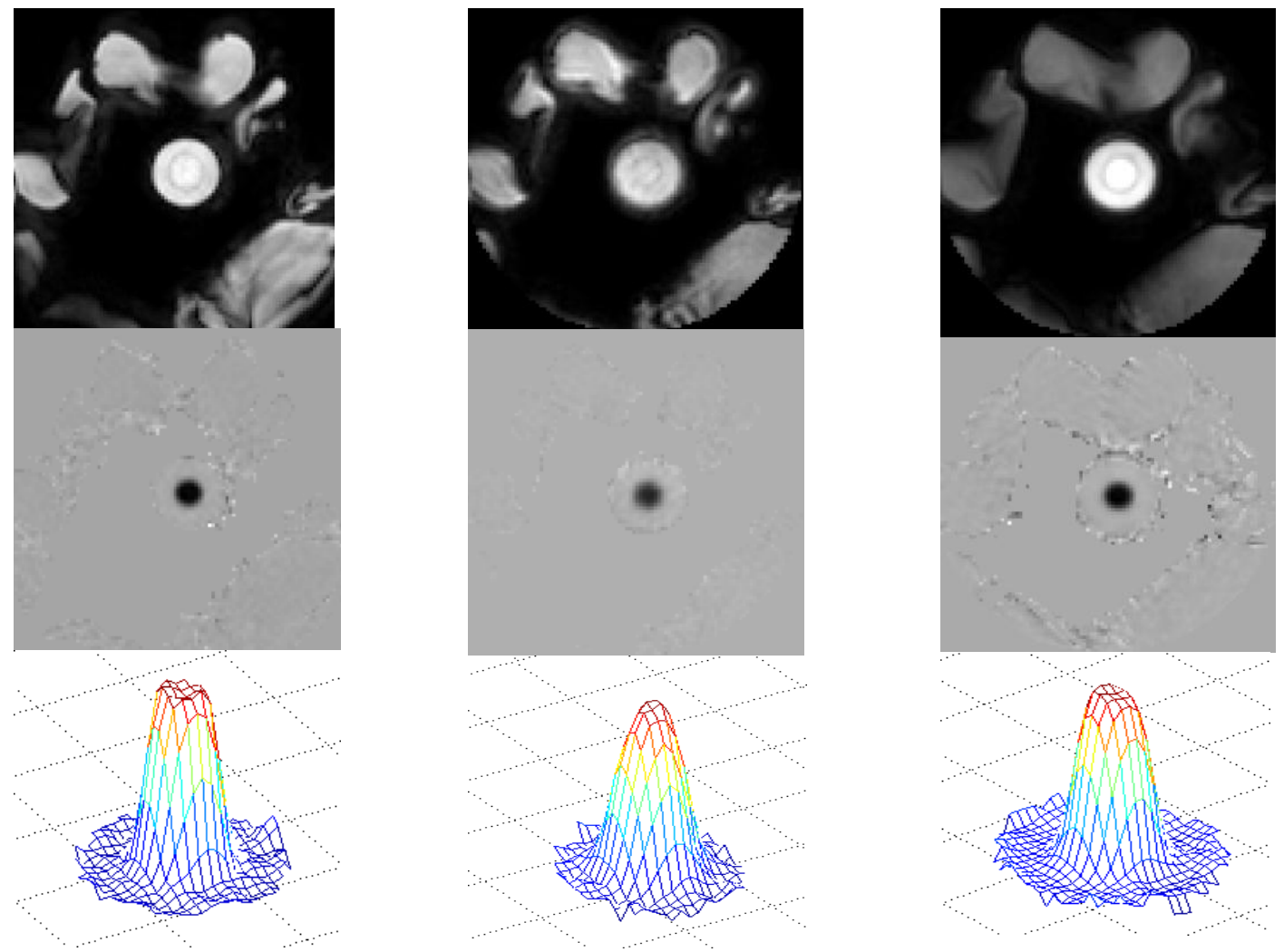

Figure 31: Magnitude image (first row), velocity image (second row), and velocity profile (third row) for medium flow rate $\mathrm{Q}=39.6 \mathrm{~mL} / \mathrm{s}$ at $33 \mathrm{~mm}$ distal to throat of the stenosis using Cartesian ( first column), spiral (second column), and 3-D UTE (third column) PC MRI sequences. 
MRI. It should be noted that in the first row, the cross Section of the phantom is circular.

Saline bags were placed around the phantom to permit the system to determine the resonance frequency. No signal loss appears in the magnitude image and velocity profiles have identical shapes. The error for measured flow in low flow rate is shown in Table 2 and result shows the least error in Cartesian trajectory. Although spiral and 3-D UTE reveal
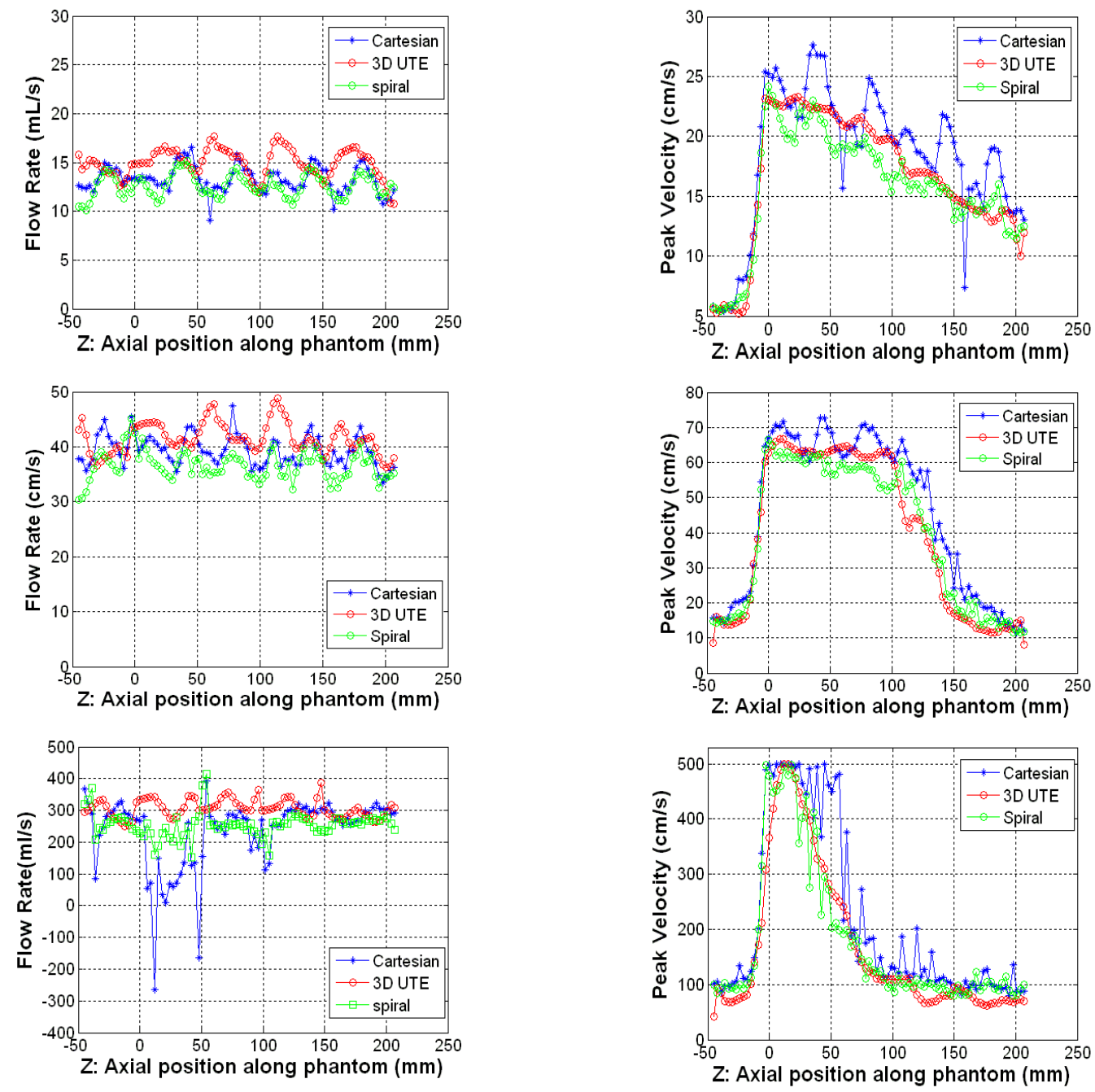

Figure 32: Measured flow rate (left column) and peak velocity ( right column) for Cartesian, spiral and 3-D UTE PC MRI sequences for low flow rate $Q=13.2 \mathrm{~mL} / \mathrm{s}$ along the length of the phantom (first row), medium flow rate $\mathrm{Q}=39.4 \mathrm{~mL} / \mathrm{s}$ (second row), and high flow rate $\mathrm{Q}=300 \mathrm{~mL} / \mathrm{s}$ (third row). $\mathrm{Z}=0$ denotes throat of the stenosis and $Z>0$ correspond to positions downstream of the stenosis as shown in Figure 29. 
more errors compared to Cartesian, the accuracy of these techniques is reasonable. It is

worth pointing out that the 3-D UTE sequences shows significant improvement in low flow rates - decreasing the error to $\sim 5 \%$; a previously proposed $2-\mathrm{D}$ UTE sequence was reported to have $\sim 27 \%$ error at $\mathrm{Q}=100 \mathrm{~mL} / \mathrm{s}$ with an average velocity of $44 \mathrm{~cm} / \mathrm{s}$ at $\operatorname{Re}=7200$ [12].

This improvement is likely due to the phase correction step as well as less phase error due to eddy current in our 3-D UTE technique. Similarly, Figure 31 demonstrates the magnitude image, flow image, and velocity profile for flow rate of $Q=39.6 \mathrm{ml} / \mathrm{s}$ using mentioned sequences. The result shows excellent resemblance between the three sequences.

Figure 32 displays the measured flow and peak velocity for the three sequences and at three flow rates along the length of the phantom. The calculated flow rates along the length of the phantom for low and medium flow rates using three sequences are in good agreement and the errors for the non-Cartesian sequences (shown in Table 2) are acceptable. In case of low and medium flow rates, the measured flow rate using Cartesian trajectory had the most accuracy compared to the generated flow rate. However, the variability of peak velocity measured for the Cartesian sequence was higher than the other two techniques. 3-D UTEPC sequence exhibits the least variability across the repeated slices.

As previously reported [9], the measured flow using the Cartesian trajectory at high flow rate exhibits significant underestimation. This can also be seen in Figure 32(third row) where measured flow and peak velocity using Cartesian trajectory at $Q=300 \mathrm{ml} / \mathrm{s}$ exhibits significant errors distal to the throat of the stenosis where a flow jet is present. Although the spiral PC sequence revealed less flow rate error and also less variability in the estimated peak 

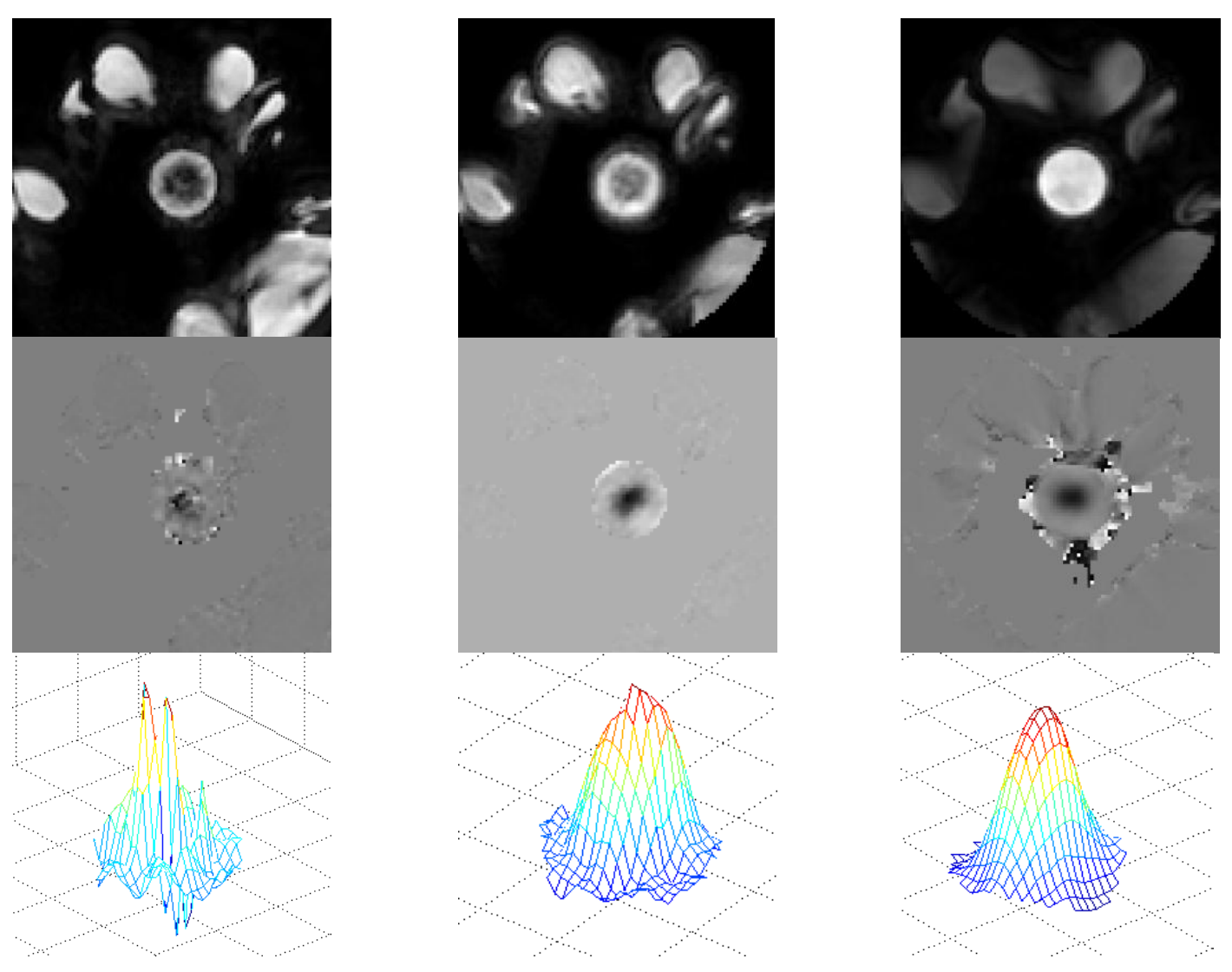

Figure 33: Magnitude image (first row), velocity image (second row), and velocity profile (third row) for high flow rate $\mathrm{Q}=300 \mathrm{~mL} / \mathrm{s}$ at $33 \mathrm{~mm}$ distal to throat of the stenosis for the case of Cartesian (first column), spiral (second column), and 3-D UTE (third column) acquisitions. The magnitudes images for Cartesian and spiral sequences show signal loss. The velocity image in the Cartesian acquisition reveals a significant phase error at the center of jet compared to spiral and 3-D UTE. There is negligible signal loss and phase error in 3D UTE PC MRI.

velocity compared to the Cartesian trajectory, the most accurate measurements were obtained with the 3-D UTE sequence.

Figure 33 displays the phantom magnitude and velocity image as well as velocity profile at high flow rate $\mathrm{Q}=300 \mathrm{~mL} / \mathrm{s}$. The magnitude image using Cartesian trajectory reveals a high signal loss while the corresponding velocity image shows significant phase error and noise at the center of jet due to the signal loss in magnitude image. Far less signal loss in magnitude image and phase error in velocity image can be seen in the spiral trajectory. This is because 
the shorter TE in the spiral sequence results in less intravoxel dephasing. It should be noted however that the TE in spiral acquisition is not short enough to entirely resolve the signal loss and phase error. On the other hand, the 3-D UTE sequence with significantly shorter TE, demonstrates no signal loss in the magnitude image and additionally, no phase error can be observed in the velocity image at the center of flow jet. The same phenomenon can also be seen in the corresponding velocity profiles. Note that the phase error in velocity image around the phantom is due to susceptibility artifact between phantom wall and surrounding air and does not affect the flow measurement.

\subsection{Pulsatile flow in normal carotid artery}

\subsubsection{Imaging Protocol}

Imaging was performed on an Achieva 1.5T Philips scanner using a combined 16-element SENSE Neurovascular coil capable of imaging carotid vessels from the aortic arch to the Circle of Willis.

For the in-vivo study, four normal volunteers with an average of $27 \pm 4$ years of age were scanned using standard multi-2-D cine PC MRI sequences as well as the proposed 3-D cine UTE-PC MRI sequence. Imaging was performed perpendicular to the common carotid

Table 3: Acquisition parameter for Cartesian PC MRI and UTE-PC MRI using stack of star

\begin{tabular}{ccc} 
& trajectories & \\
\hline Parameter & Cartesian PC MRI & 3-D UTE-PC MRI \\
\hline FOV (mm) & $160 \times 188 \times 5$ & $170 \times 170 \times 50$ \\
\hline TE (ms) & 2.9 & 1.08 \\
\hline TR (ms) & 4.9 & $1.17 \times 1.17 \times 5$ \\
\hline spatial & $2.0 \times 2.0 \times 5.0$ & 15 \\
\hline resolution(mm) & & \\
\hline Flip angle $(\mathbf{d e g r e e})$ & 200 in through plane direction & 200 in through plane direction \\
\hline Venc $\mathbf{( c m / s )}$ &
\end{tabular}


artery covering an axial 5cm 3-D volume including 10 slices with $5 \mathrm{~mm}$ slice thickness. The other parameters for the conventional and proposed technique are shown in Table 3. The multi-2-D and 3-D volume was located $1 \mathrm{~cm}$ proximal to $4 \mathrm{~cm}$ distal to the carotid bifurcation. Blood flow was evaluated in the right and left common carotid artery (CCA) proximal to carotid bifurcation as well as Internal Carotid Artery (ICA) and External Carotid Artery (ECA) distal to bifurcation.
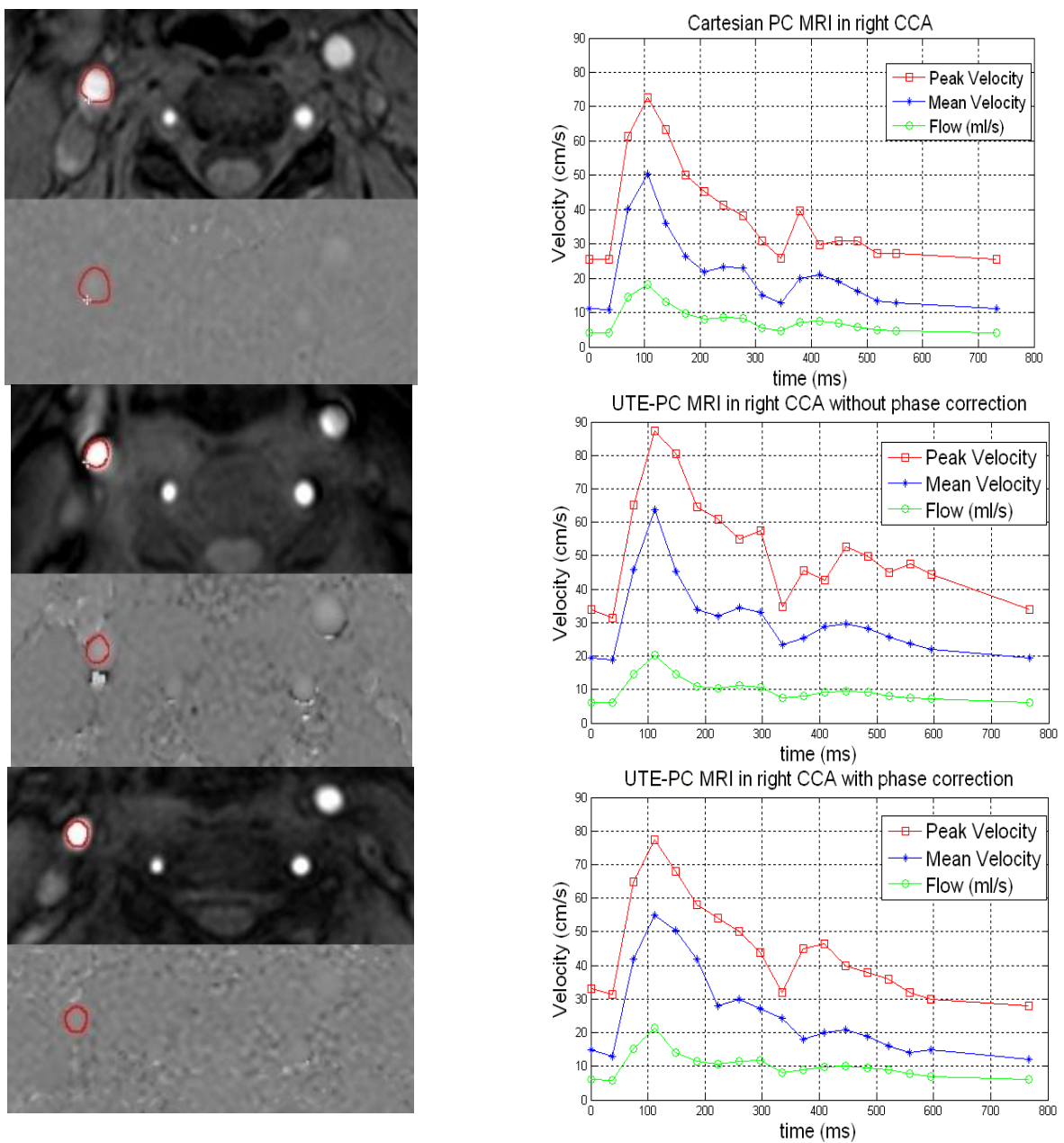

Figure 34: Right CCA magnitude and phase image (left column) and peak velocity, mean velocity and flow waveform (right column) for conventional sequence (first row) 3-D UTE-PC MRI without phase error correction (second row) and 3-D UTE-PC MRI with phase error correction (third row). Image slice from the $\mathrm{M} 2 \mathrm{D}$ acquisition in (first row) and 3-D acquisition in (second row) and (third row) was located $5 \mathrm{~mm}$ proximal to bifurcation. 
Table 4: Comparison of flow measurement between 3-D UTE and conventional PC MRI in 4 normal volunteers. Quantities have been averaged for the right CCA.

\begin{tabular}{ccccc} 
& $\begin{array}{c}\text { Multi-2D } \\
\text { Cartesian PC } \\
\text { MRI }\end{array}$ & $\begin{array}{c}\text { 3-D UTE-PC } \\
\text { MRI without } \\
\text { phase correction }\end{array}$ & $\begin{array}{c}\text { 3-D UTE-PC } \\
\text { MRI with phase } \\
\text { correction }\end{array}$ & Difference (\%) \\
\hline $\begin{array}{c}\text { Peak systolic } \\
\text { velocity (cm/s) }\end{array}$ & $72.6 \pm 11$ & $87.2 \pm 14$ & $75.3 \pm 7$ & 3.7 \\
\hline $\begin{array}{c}\text { Mean systolic } \\
\text { velocity (cm/s) }\end{array}$ & $50.3 \pm 13$ & $63.7 \pm 13$ & $54.3 \pm 11$ & 7.9 \\
\hline $\begin{array}{c}\text { Peak systolic } \\
\text { flow(mL/s) }\end{array}$ & $17.8 \pm 7$ & $21.5 \pm 8$ & $19.1 \pm 6$ & 7.3 \\
\hline
\end{tabular}

\subsubsection{Results}

\subsubsection{Phase error correction in in-vivo study}

The effect of phase error correction was investigated in an in-vivo study on a normal volunteer. Figure 34 demonstrates the peak velocity, mean velocity and flow in right CCA 5 mm proximal to bifurcation using conventional PC MRI and proposed 3-D UTE-PC MRI

with and without phase error correction. Flow waveforms and temporal evolution for peak and mean velocity for 3-D UTE-PC MRI after phase error correction have an excellent correlation with conventional technique and the difference between the peak systolic velocity using conventional and 3-D UTE-PC sequence after phase error correction is less than 4\%.

However, this difference in case of 3-D UTE-PC MRI without phase error correction is more than $10 \%$. In addition, magnitude and phase images after phase error correction show less artifact compared to the case without phase error correction. 


\subsubsection{Flow quantification in in-vivo study}

The collected 3-D volume can be investigated in two areas: (1) common carotid artery (CCA) proximal to carotid bifurcation in the right and left side of neck, and (2) internal carotid artery (ICA) and external carotid artery (ECA) distal to bifurcation in the right and left side of neck. The first area includes four slices before carotid bifurcation and the second area includes six slices at bifurcation and distal to bifurcation. Slice 5 in 3-D volume is right after carotid bifurcation and discrimination of vessels is difficult and was disregarded from further analysis.

The result of blood velocity measurement in right CCA for one slice located at $0.5 \mathrm{~cm}$ proximal to bifurcation for all volunteers using standard multi-2D PC MRI and 3-D UTEPC MRI before and after phase error correction is summarized in Table 4. The last column in Table 4 represents the difference of measured quantities between conventional PC MRI and 3-D UTE-PC MRI after phase error correction.

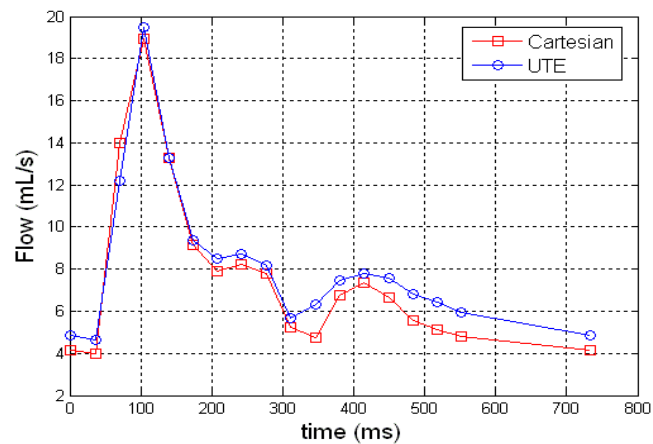

(a)

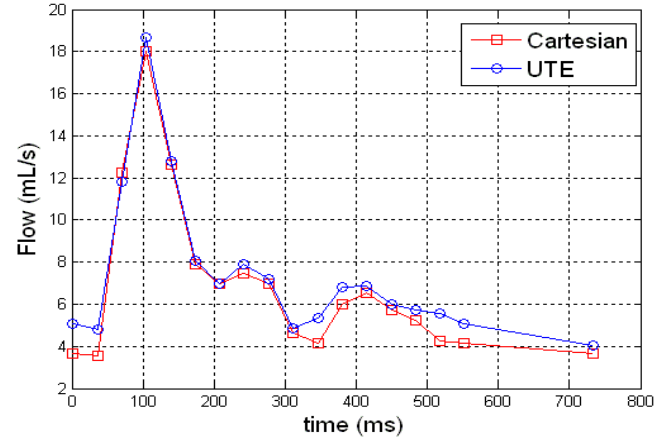

(b)

Figure 35: The average blood flow in right (a) and left (b) CCA using multi-2D PC MRI (red line) and 3-D UTE-PC MRI after phase error correction (blue line). 


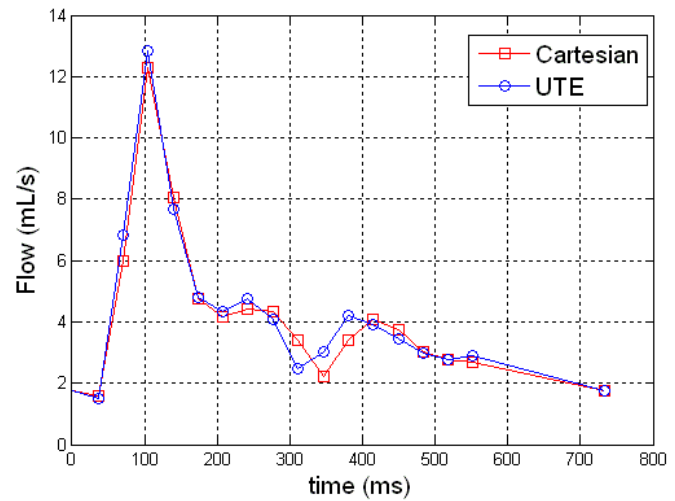

(a)

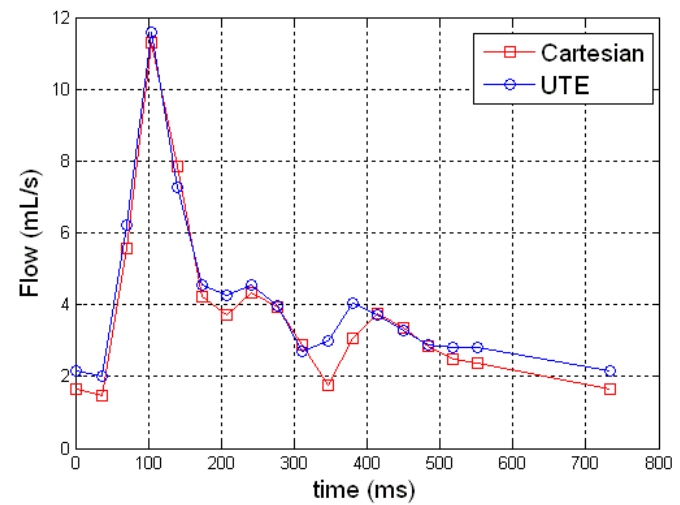

(b)

Figure 36: The average blood flow in right (a) and left (b) ECA using multi-2D PC MRI (red line) and 3-D UTE-PC MRI after phase error correction (blue line).

The velocity and flow measurements for 3-D UTE-PC MRI after phase error correction are different from conventional PC MRI by less than $8 \%$ while this difference falls to $20 \%$ when there is no phase error correction.

The average blood flow in right and left CCA for four slices proximal to carotid bifurcation in all volunteer was shown in Figure 35. The blood flows were acquired using standard multi-2D PC MRI and 3-D UTE-PC MRI after phase error correction. There is reasonable agreement between flow waveform acquired using two sequences in left and right CCA. The flow waveforms in multi-2D PC MRI and 3-D UTE-PC MRI have a small temporal shift which is due to different gating techniques in two collected scans and subsequently slightly different time in corresponding cardiac frames.

In addition, the average flow waveforms for left and right ICA and left and right ECA in five slices distal to carotid bifurcation in all volunteers are shown in Figures 36 and 37. The flow waveforms are in excellent agreement between standard multi-2D PC MRI and 3-D UTEPC MRI after phase error correction. 


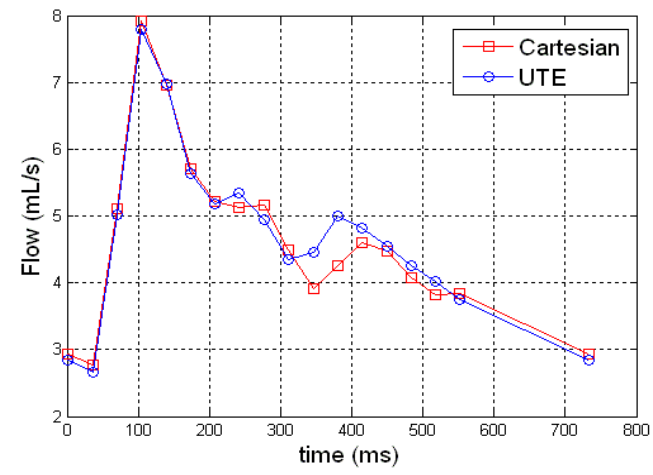

(a)

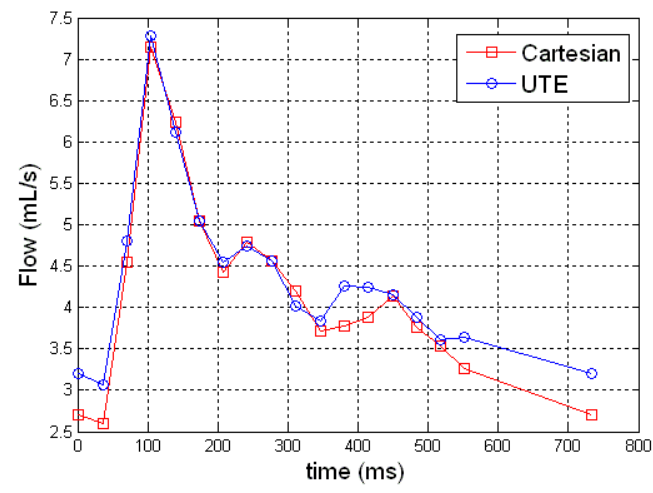

(b)

Figure 37: The average blood flow in right (a) and left (b) ICA using multi-2D PC MRI (red line) and 3-D UTE-PC MRI after phase error correction (blue line).

Table 5: Mean square error (MSE) in flow measurement using 3-D UTE-PC MRI after phase error correction compared to conventional PC MRI

\begin{tabular}{ccccccc}
\hline & $\begin{array}{c}\text { MSE in } \\
\text { right CCA }\end{array}$ & $\begin{array}{c}\text { MSE in } \\
\text { right ICA }\end{array}$ & $\begin{array}{c}\text { MSE in } \\
\text { right ECA }\end{array}$ & $\begin{array}{c}\text { MSE in left } \\
\text { CCA }\end{array}$ & $\begin{array}{c}\text { MSE in } \\
\text { left ICA }\end{array}$ & $\begin{array}{c}\text { MSE in left } \\
\text { ECA }\end{array}$ \\
\hline $\begin{array}{c}\text { 3-D UTE-PC } \\
\text { MRI }\end{array}$ & 6.68 & 3.6 & 4.1 & 5.32 & 3.8 & 4.8 \\
\hline
\end{tabular}

In order to compare the proposed 3-D UTE-PC MRI technique with conventional multi2-D PC MRI sequence, the normalized mean square error (MSE) is calculated. The normalized root mean square error can be expressed as:

$$
\text { Error }=\sqrt{\frac{1}{\mathrm{~N}}\left(\frac{\mathrm{Q}_{\mathrm{cart}}-\mathrm{Q}_{\mathrm{UTE}}}{\mathrm{Q}_{\mathrm{cart}}}\right)^{2}}
$$

where $\mathrm{Q}_{\text {cart }}$ and $\mathrm{Q}_{\mathrm{UTE}}$ describe measured flow using Conventional PC MRI and 3-D UTEPC MRI technique respectively and $\mathrm{N}$ is the number of time points in a cardiac cycle where data is collected (i.e. number of cardiac phases).

Table 5 demonstrates the calculated error using 3-D UTE-PC MRI after phase error correction compared to conventional PC MRI. This Table shows that the measured errors 
for all the vessels are less the 7\% in case of healthy volunteers. This accuracy in flow measurement is compatible with our hypothesis that proposed 3-D UTE-PC MRI technique lead to acceptable flow quantification with respect to conventional technique in normal volunteer. 


\section{3-D UTE PC MRI: THE EFFECT OF VENC, SAMPLING DENSITY, GRADIENT STRENGTH, AND SLEW RATE}

\subsection{Introduction}

In general, 3 Tesla scanners benefit from higher Signal to Noise Ratio (SNR) compared to 1.5 Tesla. In this Chapter, the 3-D cine UTE-PC MRI technique was investigated at 3 T scanners to potentially adopt this advantage to improve the flow assessment. The accuracy of flow assessment in MRI scanner is highly dependent upon scan parameters. This is more important in case of UTE sequence and parameters such as gradient strength, slew rate, Velocity encoding (Venc), Echo Time (TE), and sampling density of radial k-space lines which can significantly affect the robustness of flow quantification.

3D volumetric scans usually suffer from long scan times. This issue is more problematic in radial acquisitions due to necessity of acquiring more k-space lines compared to Cartesian acquisition. Under-sampling of radial k-space lines results in shorter scan time in 3-D UTE PC MRI sequence. However, excessive under-sampling of k-space lines potentially leads to artifacts and inaccuracy of flow quantification.

In this Chapter, the accuracy of flow quantification using various scan parameters will be investigated. The sequence was utilized to quantitatively measure blood velocity in the carotid bifurcation and results were compared to conventional PC MRI sequences. 


\subsection{Gradient strength and slew rate}

Gradient strength and the rate of gradient strength variation are two major factors affecting the length of bipolar gradients. The slew rate of a gradient is defined as the rate of change of gradient amplitude and can be expressed as

$$
\mathrm{SR}=\frac{\mathrm{G}_{\mathrm{max}}}{\mathrm{T}}
$$

where $G_{\text {max }}$ is maximum amplitude of gradient and $\mathrm{T}$ is the duration that gradient amplitude reaches from zero to the maximum amplitude. Figure 38 demonstrates how the variation of slew rate and changing the gradient strength from $10 \mathrm{mT} / \mathrm{sec}$ to $20 \mathrm{mT} / \mathrm{sec}$ Vlead to shorter bipolar gradient length and consequently shorter TE.

The minimum achievable TE using 3-D UTE-PC MRI at 1.5T for Venc=200 was reported as $1.1 \mathrm{msec}[16]$. To achieve this TE, the maximum gradient field strength and slew rate are set to $10 \mathrm{mT} / \mathrm{sec}$ and $100 \mathrm{mT} / \mathrm{m} / \mathrm{msec}$, respectively. Benefiting from gradient

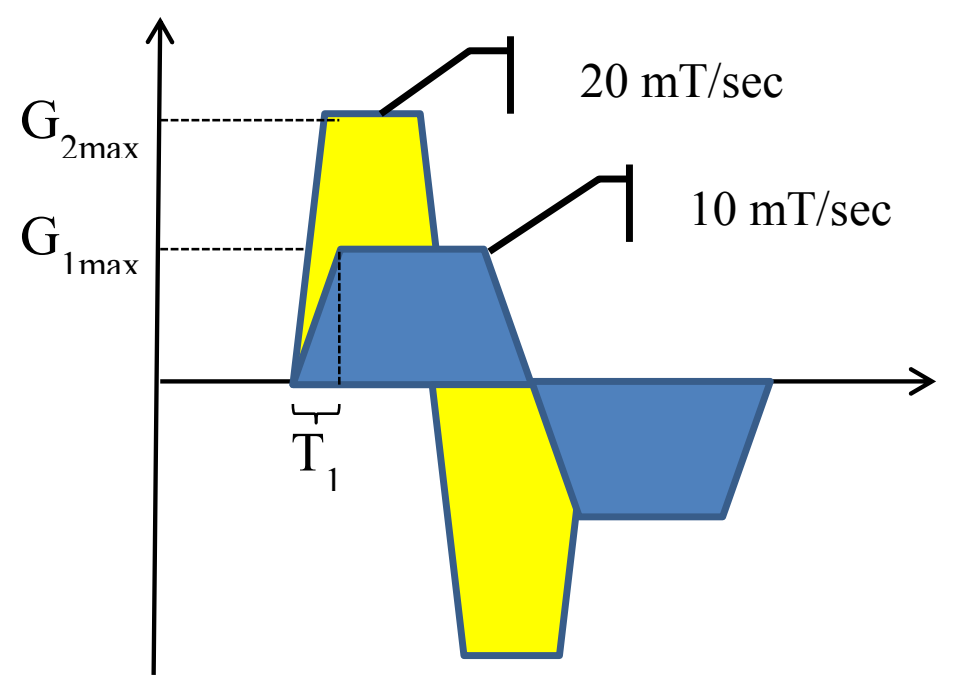

Figure 38: A schematic of bipolar gradients in PC MRI. Blue shaded gradient has $10 \mathrm{mT} / \mathrm{sec}$ strength and yellow shaded gradient benefits from higher gradient $20 \mathrm{mT} / \mathrm{sec}$ and shorter length. 
strength increase in 3T, higher gradient strength and slew rates were utilized (as is shown in Table 6) in order to further reduce the TE.

\subsection{Center-out radial spoke number variation}

The scan time for the UTE-PC MRI sequence is longer than conventional Cartesian PC MRI. This is due to necessity for $\pi \mathrm{N} \mathrm{k}$-space line in the UTE technique to cover the whole $\mathrm{k}$-space to acquire an $\mathrm{N} \times \mathrm{N}$ image, whereas with the Cartesian technique, $\mathrm{N}$ k-space lines are sufficient for producing an $\mathrm{N} \times \mathrm{N}$ image. In addition, 3-D trajectory acquisition would make the scan time even longer. Therefore, it is crucial to reduce the scan time, especially for in-vivo study. One benefit of radial acquisition is that it is possible to under-sample the kspace lines without the need to apply fast imaging techniques such as parallel imaging or partial k-space acquisition. The effect of sampling the radial k-space lines (reducing the

Table 6: Acquisition parameter for conventional 3D PC MRI and 3D UTE-PC MRI using different scan

\begin{tabular}{|c|c|c|c|c|}
\hline & & $\begin{array}{c}\text { Variable } \\
\text { parameter }\end{array}$ & TE/TR & Scan time \\
\hline \multirow{12}{*}{ 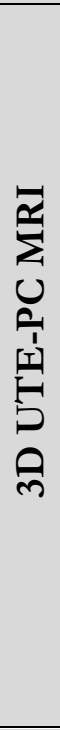 } & \multirow{4}{*}{$\begin{array}{c}\text { sampling } \\
\text { (Num of spokes) }\end{array}$} & 25 & $1.3 / 5.5$ & $1: 13$ \\
\hline & & 50 & $1.3 / 5.5$ & $2: 24$ \\
\hline & & 75 & $1.3 / 5.5$ & $3: 35$ \\
\hline & & 100 & $1.3 / 5.5$ & $4: 47$ \\
\hline & \multirow{5}{*}{$\begin{array}{c}\text { (Gradient strength/ } \\
\text { Slew rate) }\end{array}$} & L1 $(12 / 25)^{*}$ & $2.2 / 9.1$ & $7: 09$ \\
\hline & & $\mathrm{L} 2(10 / 25)^{*}$ & $2.4 / 9.3$ & $7: 09$ \\
\hline & & L3(18/52)* & $1.6 / 6.6$ & $4: 18$ \\
\hline & & $\mathrm{L} 4(21 / 100)^{*}$ & $1.3 / 5.5$ & $3: 35$ \\
\hline & & $\mathrm{L} 5(40 / 200)^{*}$ & $1.0 / 5.0$ & $3: 35$ \\
\hline & \multirow{3}{*}{ Venc } & 150 & $1.3 / 5.5$ & $3: 35$ \\
\hline & & 200 & $1.2 / 5.3$ & $3: 35$ \\
\hline & & 250 & $1.1 / 5.0$ & $3: 35$ \\
\hline \multicolumn{2}{|r|}{ Conventional PC MRI } & - & $4.0 / 7.3$ & $3: 33$ \\
\hline
\end{tabular}


number of MR projections) on the accuracy of flow quantification using proposed 3-D UTE-PC MRI can be seen in Table 7.

\subsection{Imaging strategy}

Imaging was performed on an Achieva 3T Philips scanner using a 16-element SENSE Neurovascular coil capable of imaging carotid vessels from the aortic arch to the Circle of Willis. For the in-vivo study, three normal volunteers with an average of $35 \pm 5$ years of age were scanned. 3D Volume was located perpendicular to the common carotid artery, $1 \mathrm{~cm}$ proximal to $4 \mathrm{~cm}$ distal to the carotid bifurcation, covering 10 slices with $5 \mathrm{~mm}$ slice thickness of each slice. Two sequences were employed: i) conventional 3-D Cine PC MRI sequence with Cartesian trajectory and 3-D UTE-PC sequence with center-out radial trajectory. The common parameters among all scans are: $\mathrm{FOV}=160 * 160 * 50 \mathrm{~mm}$, flip

Table 7: Flow assessment in carotid artery using conventional 3D PC MRI and 3D UTE PC MRI with various scan parameters

\begin{tabular}{|c|c|c|c|c|c|}
\hline & \multirow{2}{*}{$\begin{array}{c}\text { Variable } \\
\text { parameter }\end{array}$} & \multicolumn{2}{|c|}{$\begin{array}{c}\text { Systolic flow error } \\
(\%)\end{array}$} & \multicolumn{2}{|c|}{$\begin{array}{c}\text { Systolic mean vel error } \\
\qquad(\%)\end{array}$} \\
\hline & & CCA & ICA & CCA & ICA \\
\hline \multirow{4}{*}{$\begin{array}{l}\text { Sampling } \\
\text { (Num of } \\
\text { spokes) }\end{array}$} & 25 & 12.3 & 15.3 & 17.2 & 19.9 \\
\hline & 50 & 7.8 & 9.9 & 11.7 & 13.7 \\
\hline & 75 & 6.9 & 9.4 & 9.2 & 10.3 \\
\hline & 100 & 9.4 & 11.7 & 13.3 & 14.1 \\
\hline \multirow{5}{*}{$\begin{array}{l}\text { (Gradient } \\
\text { strength/ } \\
\text { Slew rate) }\end{array}$} & $\mathrm{L1}(12 / 25)^{*}$ & 13.3 & 18.3 & 15.8 & 18.8 \\
\hline & $\mathrm{L} 2(10 / 25)^{*}$ & 15.6 & 30.1 & 11.6 & 30.7 \\
\hline & L3(18/52)* & 4.2 & 15.2 & 6.7 & 16.2 \\
\hline & $\mathrm{L} 4(21 / 100)^{*}$ & 6.9 & 9.4 & 9.2 & 10.3 \\
\hline & L5(40/200)* & 13.4 & 27.3 & 18.7 & 28.2 \\
\hline \multirow{3}{*}{ Venc } & 150 & 6.9 & 9.4 & 9.2 & 10.3 \\
\hline & 200 & 14.7 & 20.6 & 12.0 & 21.2 \\
\hline & 250 & 21.4 & 53.2 & 18.1 & 54.4 \\
\hline $\begin{array}{l}\text { Conventional } \\
\text { PC }\end{array}$ & - & - & - & - & - \\
\hline
\end{tabular}


angle $=10$, spatial resolution $=1.5 * 1.5 * 5.0 \mathrm{~mm}$, and 15 cardiac phases. Other parameters were shown in Table 6.

\subsection{Results and discussions}

Blood flow was evaluated in three normal subjects in the right and left common carotid artery (CCA) proximal to carotid bifurcation and the right and left Internal Carotid Artery (ICA) distal to bifurcation. Table 7 demonstrates the results of systolic flow and mean velocity errors provided by comparison between 3-D UTE PC MRI and conventional 3D PC MRI in right CCA ( average over 9 slices) and ICA (averaged over 15 slices) of three normal volunteers.

By sampling of radial k-space line with $25 \%$ of complete $\mathrm{k}$-space, the scan time was three times shorter than Cartesian sequence. However, the errors are higher than other sampling rate. $50 \%$ and $75 \%$ sampling showed acceptable errors with shorter scan time compared to complete k-space. Surprisingly, complete k-space acquisition results in higher errors compared to $75 \%$ which possibly is due to oversampling of center of $\mathrm{k}$-space leading to overestimation of flow. The smallest errors in CCA and ICA using various sampling factors were achieved using $75 \%$ of sampling.

The results of gradient strength and slew rate variation show that L3 and L4 gradient strength achieve optimized errors with short $\mathrm{TE}$ and similar scan time as Cartesian technique. The shortest TE can be achieved with L5 gradient in which gradient strength and slew rate were increased to $40 \mathrm{mT} / \mathrm{m}$ and $200 \mathrm{mT} / \mathrm{m} / \mathrm{mse}$, respectively. However, the errors were increased due to higher eddy current and consequent phase error. It is worthwhile to 
note that, lower gradient strength and slew rate does not necessarily result in less error. This can be interpreted due to longer TE/TR obtained using these sequences.

Variation of Venc revealed that by increasing the Venc beyond maximum actual velocity in artery the measured errors were significantly increased. The best result was achieved using Venc 150 while increasing the Venc causes a significant overestimation of mean flow and velocity.

Error! Reference source not found. demonstrates the flow waveforms for sequences ith various parameters in right CCA and ICA in one cardiac cycle using conventional PC MRI (red curve) and 3D UTE PC MRI. The Figure shows the best agreement between 3D UTE and conventional PC MRI flow waveforms can be achieved with following parameters: Venc $=150$ (blue waveform in left image), L3 gradient strength (sky-blue in middle image), and $75 \%$ sampling of $\mathrm{k}$-space lines (sky-blue in right image).
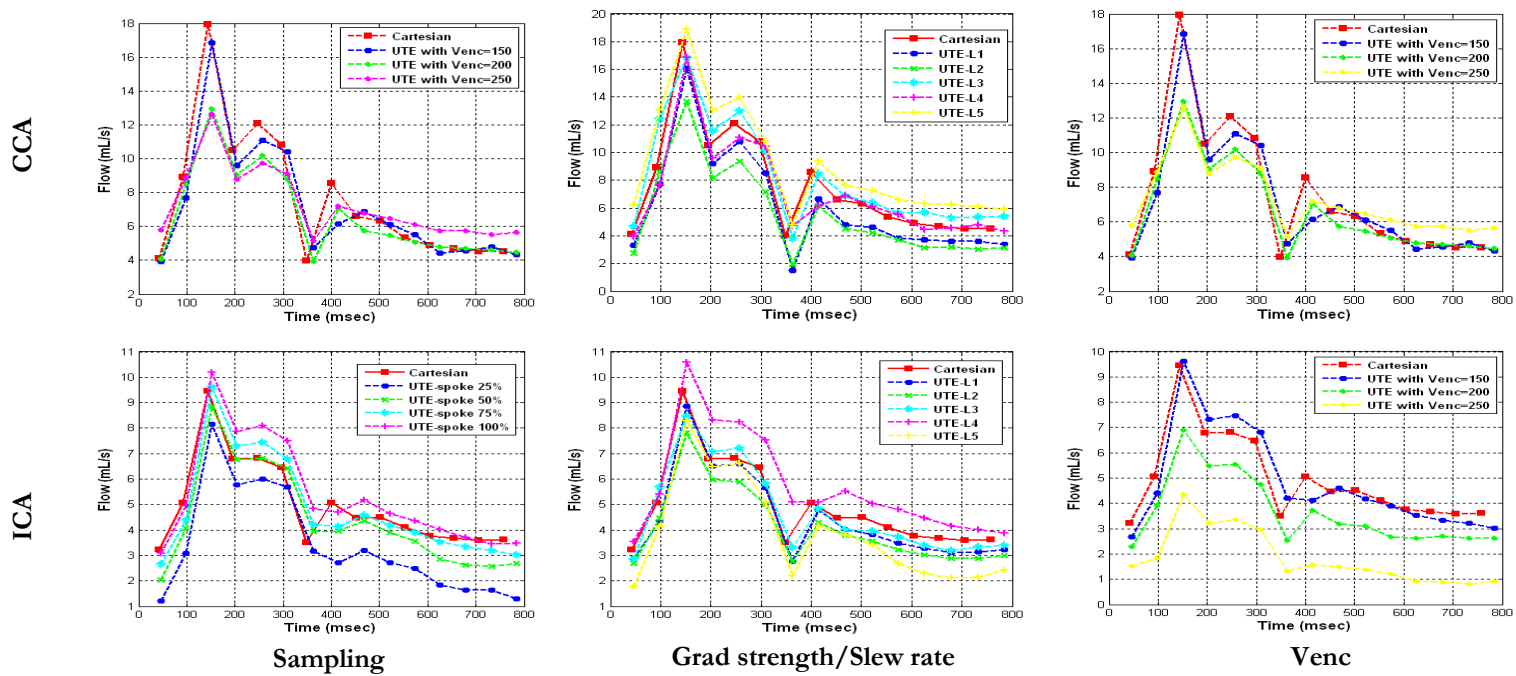

Figure 39: Flow waveform in right CCA (top row) and ICA (bottom row) using conventional 3D PC MRI and 3D UTE PC MRI with various sampling, gradient strength, slew rate, and Venc. 


\section{REDUCED TE 4D FLOW SPIRAL MRI FOR ASSESSMENT OF FLOW AND HEMODYNAMICS}

\subsection{Introduction}

Conventional 2-D PC MRI is based on flow quantification in a slice of interest in a 2-D image. Although it is possible to acquire 3 components of velocities in a $2 \mathrm{D}$ slice, by extending the imaging volume to 3-D, 3 components of flow can be determined in 3D. 4-D flow imaging is based on flow quantification in three directions in subsequent TR's in a 3-D volume. 4-D flow MRI has been widely investigated to quantify and visualize the global and local blood flow in arteries with complex flow patterns [111, 153-159]. In addition to providing comprehensive anatomical and flow information in both in-plane and throughplane directions, 4D flow imaging results in shorter total scan time compared to 3-D flow imaging or multiple $2 \mathrm{D}$ slice imaging to cover a volume; in either case, a separate scan for each of the 3 flow directions is required.

4D flow MRI is a powerful technique for quantitative flow assessment and visualization of complex flow patterns and hemodynamics of cardiovascular flows. This technique results in more anatomical information and comprehensive assessment of blood flow. However, conventional 4D PC MRI suffers from a few obstacles for clinical applications. The total scan time is long, especially in large volumes with high spatial resolutions. Inaccuracy of conventional Cartesian PC MRI in the setting of atherosclerosis and in general, disturbed and turbulent blood flow is another important challenge. This inaccuracy is the consequence 
of signal loss, intravoxel dephasing and flow-related artifact in the presence of disturbed and turbulent flow. Spiral k-space trajectory has valuable attributes which can help overcome some of the problems with 4D flow Cartesian acquisitions. Spiral trajectory has several desirable characteristics compared to Cartesian trajectory and has been previously been employed for several clinical studies $[5,160]$. The readout echo time (TE) in spiral trajectory is shorter due to removing the rephasing part of readout gradient leading to shorter scan time. In addition, signal to noise ratio (SNR) is higher and it benefits from desirable flow characteristic [161].

In addition to longer acquisition times, atherosclerotic disease and vascular occlusions cause challenges to conventional PC acquisitions due to intravoxel dephasing secondary to disturbed blood flow and turbulence distal to narrowing, often resulting in signal loss and flow-related artifacts $[5,47,162]$. Several approaches have been developed to mitigate the signal loss and flow-related artifacts in PC-MRI [4, 6, 7]. However, a reliable flow measurement technique in the presence of turbulence has remained elusive. One important approach that has revealed significant impact in correction of the signal loss involves reduction of the echo time (TE) and gradient duration $[150,163]$. Reducing the TE decreases the impact of turbulent fluctuation velocity, intravoxel dephasing and the subsequent signal loss. The approach results in higher signal to noise ratio and more reliable estimation of disturbed and jet flows since a shorter TE will ameliorate the effect of intravoxel dephasing caused by random fluid mixing. In addition to scan efficiency, spiral acquisitions with shorter TE have the potential to reduce the signal loss and flow-related artifacts and improve the accuracy of flow quantification. 
Previously, Sigfridsson et al. [164] proposed a 4D Spiral flow sequence and used their method for imaging and visualization of flow through the aorta. In this Chapter, a 4D spiral flow MRI technique was designed which through combination of bipolar and slice select gradient further reduces the TE in comparison to Sigfridsson et al.'s implementation. Carotid artery was selected for study due to irregular geometry of this vessel at carotid bifurcation which results in disturbed blood flow. Flow assessment using conventional 4D PC MRI can yield inaccurate results in the presence of atherosclerostic disease in carotid bifurcation and internal carotid artery (ICA). The purpose of this study was to validate 4D flow spiral MRI with conventional 4D flow Cartesian MRI in normal volunteers. 4D flow spiral MRI has the advantage of having a shorter TE and total scan time when compared with conventional 4D flow acquisitions.

In this Chapter, the accuracy of flow assessment and flow visualization with reduced TE 4D Spiral PC will be investigated.

\subsection{Material and method}

\subsubsection{Pulse sequence}

Figure 40 shows the proposed sequence based on the four-point acquisition technique where flow encoding gradients are applied in all three directions in subsequent TR's [165]. For each time frame, three separate flow encoded scan (each of which with flow encoding only in one of $\mathrm{x}, \mathrm{y}$, and $\mathrm{z}$ direction) and one flow compensated (reference) is acquired. The flow velocity volumes are determined by subtraction of each flow encoded volume from the flow compensated volume. 


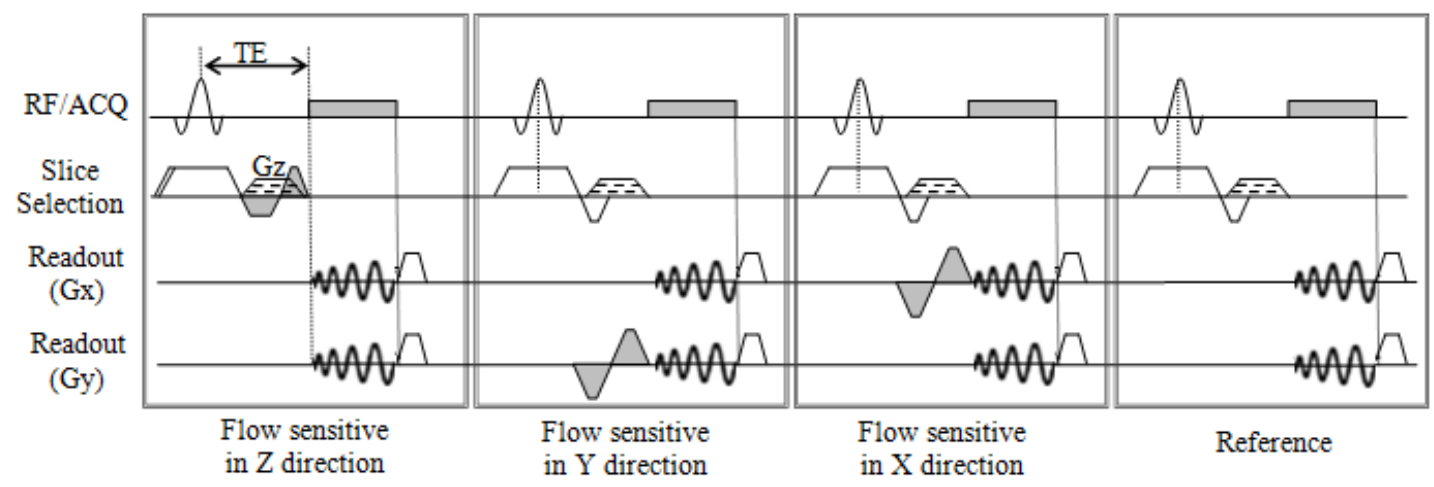

Figure 40: A schematic of reduced TE 4D spiral PC MRI sequence with for consecutive scans including 3 flow sensitive scans in three flow directions and one reference scan. Subtraction of each phase image of flow sensitive scan from phase image of the reference scan results in PC velocities for the corresponding flow direction. Gradient $G_{z}$ is applied to acquire multiple slices in a volume using stack of star strategy.

In contrast to Cartesian acquisition where k-space is acquired using parallel horizontal lines, spiral acquisition collects the k-space data using a spiral trajectory (Figure 41), significantly reducing the total scan time. The single shot spiral acquisition technique covers the entire $\mathrm{k}$-space in one readout resulting in longer TR, but with the potential drawback of off-resonance artifact due to $\mathrm{T}_{2}$ effects. Multi-shot or interleaved spiral acquires multiple spiral shots in each TR (Figure 41(b)). As a result, the whole k-space is collected using

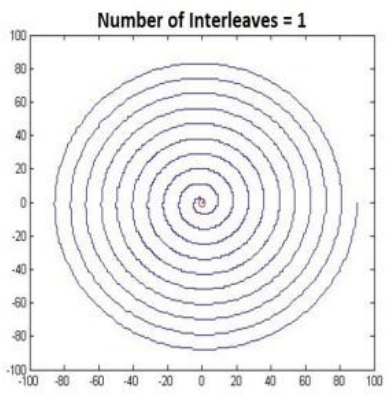

(a)

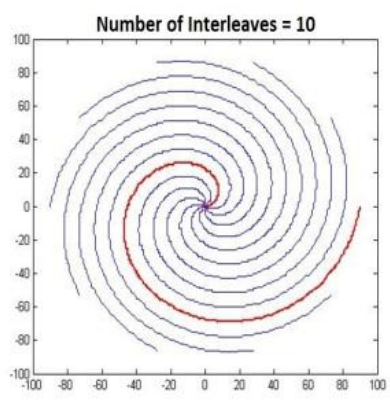

(b)

Figure 41: Demonstration of (left) conventional single shot spiral acquisition with 10 rotations and (right) interleaved spiral acquisition with 10 interleaves. 
multiple but shorter spiral arms.

In Cartesian PC sequences, slice excitation, rephasing, and velocity encoding gradients need to be applied during TE. These gradients prolong TE and may lead to phase errors and velocity miscalculation. In addition to reduced acquisition times, spiral trajectory benefits from shorter TE due to removal of the phase encoding gradient and the rephasing portion of the readout gradient. The TE in spiral acquisition is the time from the center of RF pulse to the beginning of spiral readout gradient.

Figure 40 displays the proposed 4D spiral flow sequence, designed using a 3D stack of spirals trajectory. A stack of spirals trajectory was also adopted in [164]; however, as shown in Figure 40, the TE is now further shortened through combination of the flow encoding/compensated gradient with the refocusing portion of slice select gradient. Further reduction in TE is possible using higher values of Velocity encoding (Venc).

\subsubsection{Imaging strategy}

Imaging was performed on a Philips Achieva 1.5T scanner (Philips Healthcare, Best, NL) using a combined 16-element SENSE Neurovascular coil capable of imaging carotid vessels from the aortic arch to circle of willis. Four normal volunteers with a mean age of $27 \pm 4$ years were scanned using standard 4D flow Cartesian PC sequences as well as the proposed 4D flow spiral PC sequence. Flow assessment was performed in the carotid arteries in an axial 3D volume with 10 slices and a 3D slab thickness of $5 \mathrm{~mm}$ for each slice. The acquired volume started at $15 \mathrm{~mm}$ proximal to the bifurcation and ended at $35 \mathrm{~mm}$ distal to the bifurcation. 
Phase errors due to eddy current and hardware imperfections were corrected using a static phantom which was scanned with identical parameters prior to the main scan. The phase error in static phantom MR image was assumed to be only due to eddy current and hardware imperfection and was subtracted from the in-vivo scan.

The scan parameters for two sequences were TE/TR $=4.4 / 7.7 \mathrm{~ms}$ (for Cartesian trajectory), $\mathrm{TE} / \mathrm{TR}=2.1 / 9.3 \mathrm{~ms}$ (for spiral trajectory), $\mathrm{FOV}=160 * 160 * 50 \mathrm{~mm}, \mathrm{Venc}=150$ in all three flow directions, flip angle $=10$, spatial resolution $=1.5 * 1.5 * 5.0 \mathrm{~mm}$, and 12 cine frames in each cardiac cycle. For the spiral trajectory, various combinations of spiral interleaves $(20,30,50,60)$ and readout duration $(2,3,5,10$, msec) were examined and it was determined that number of interleaves $=30$ and readout duration $=5 \mathrm{msec}$ resulted in a good compromise between flow measurement fidelity and total scan time. The scan time for the 4D Cartesian flow was 6:16 minutes while the scan time for the proposed 4D spiral flow was 3:31 minutes. The TE (2.1 $\mathrm{msec})$ and scan time (3:31 minutes) in spiral sequence shows significant improvement relative to Cartesian sequence and with the scan time reduced by half.

\subsection{Results and discussions}

Flow assessment was carried out in right and left CCA for three slices located proximal to bifurcation as well as five slices distal to bifurcation in the right and left ICA in 4 volunteers. The difference in heart rate in all volunteers was taken care of by interpolating the measured flow quantities in the last three volunteers to match their cardiac frame time with the cardiac frame time in the first volunteer. Figure 42 demonstrates the flow waveforms in right CCA averaged in 12 and right ICA averaged in 20 slices in 4 volunteers. The resulting flow 


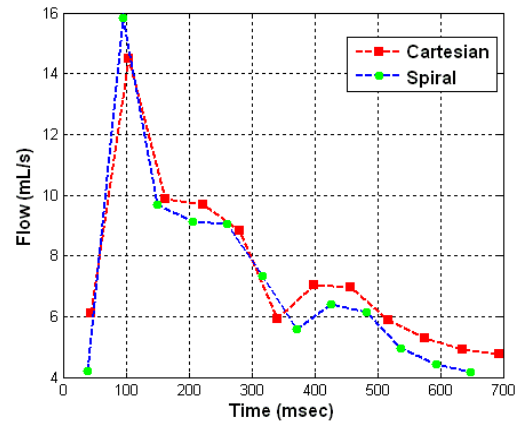

(a)

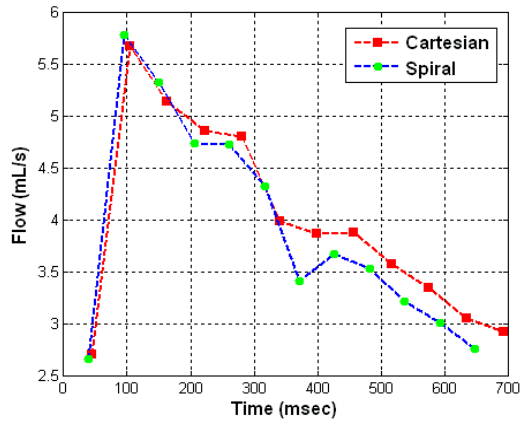

(b )

Figure 42: Flow waveform for Right CCA (a) averaged in 12 slices proximal to bifurcation and for Right ICA (b) in 20 slices distal to bifurcation using Cartesian ( red cruve) and spiral (blue curve) PC MRI in four volunteers.

waveforms for conventional 4D flow and proposed 4D spiral flow reveal good agreement in flow quantification during the entire cardiac cycle. The results of blood velocity and flow

evaluation in right and left CCA in 12 slices proximal to bifurcation and right and left ICA in 20 slices distal to the bifurcation using 4D Cartesian flow 4D spiral flow are summarized in Table 8. The values represent the difference of measured quantities between conventional and spiral flow. In order to quantitatively compare results between 4D spiral flow with the conventional technique, the normalized root mean square error (RMSE) is calculated. The normalized root mean square error can be expressed as:

$$
\text { Error }=\sqrt{\frac{1}{N}\left(\frac{Q_{\text {cart }}-Q_{S P}}{Q_{\text {cart }}}\right)^{2}}
$$

where $\mathrm{Q}_{\text {cart }}$ and $\mathrm{Q}_{\mathrm{SP}}$ are measured flow using Conventional and spiral techniques and $\mathrm{N}$ is the number of time points in a cardiac cycle where data is collected (i.e. number of cardiac phases). The velocity and flow measurements between the two show less than $10 \%$ difference for both the CCA and the ICA. The discrepancy for left and right CCA shows a reasonable accuracy with 4D spiral flow while the discrepancies for the right and left ICA are 
Table 8: Comparison of 4D Cartesian flow and 4D spiral flow in 12 slices in right and left CCA and 20 slices in right and left ICA in four volunteers. The quantities are percentage of discrepancy in spiral technique relative to Cartesian technique

\begin{tabular}{cccc}
\hline & $\begin{array}{c}\text { Peak Systolic flow } \\
\text { discrepancy }\end{array}$ & $\begin{array}{c}\text { Systolic mean velocity } \\
\text { discrepancy }\end{array}$ & $\begin{array}{c}\text { Average flow } \\
\text { discrepancy }\end{array}$ \\
\hline Right CCA & 3.7 & 3.8 & 6.3 \\
\hline Right ICA & 4.5 & 4.9 & 6.8 \\
\hline Left CCA & 5.7 & 5.9 & 9.5 \\
\hline Left ICA & 9.2 & 8.4 & 9.7 \\
\hline
\end{tabular}

slightly higher -- most likely due to smaller size of these arteries and more sensitivity to artifacts.

Figure 43 demonstrates the Bland-Altman plot representing the mean difference of flow measured using 4D Cartesian flow and 4D spiral flow. Mean flow in each slice and in each cardiac phase in 4 volunteers generated 144 data points in CCA and 240 data points in ICA.

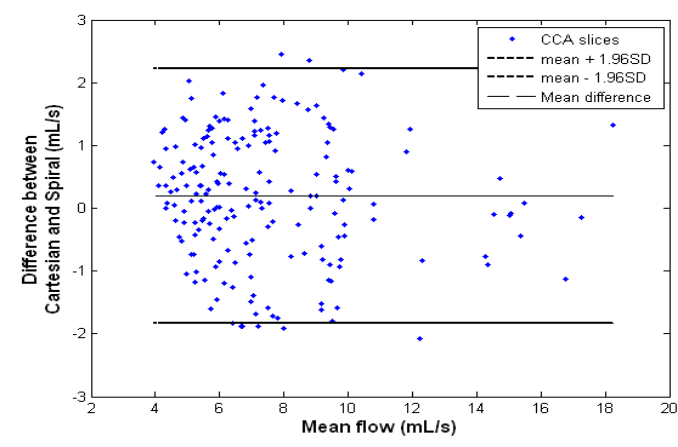

(a)

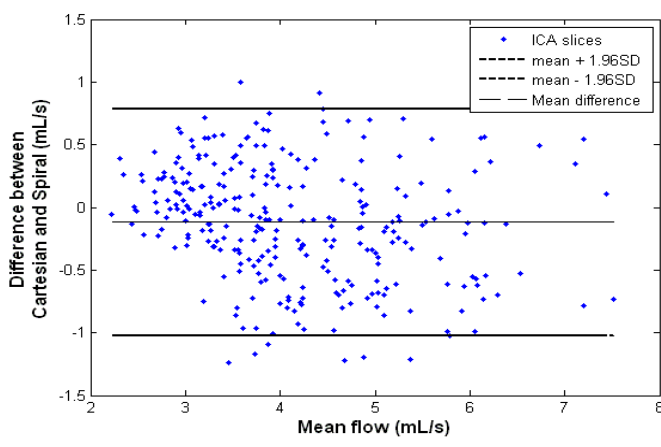

(b)

Figure 43: Bland-Altman plot in each cardiac phase in four normal volunteers demonstrating the mean flow difference between 4D conventional and 4D spiral flowin (a)RCCA and (b) LCCA. The blue dots are all measured flow values from 4 volunteers in all cardiac frames. Having 4 volunteers, 3 CCA slices in each volunteer, and 12 cardiac frames in each CCA results in $4 * 3 * 12=144$ data points (a). For ICA 5 slices are available distal to bifurcation in each volunteer and the number of data points is $4 * 5 * 12=240$ (b). The abscissa is the mean flow values from $4 \mathrm{D}$ conventional flow calculated over 12 slices during the cardiac cycle. 


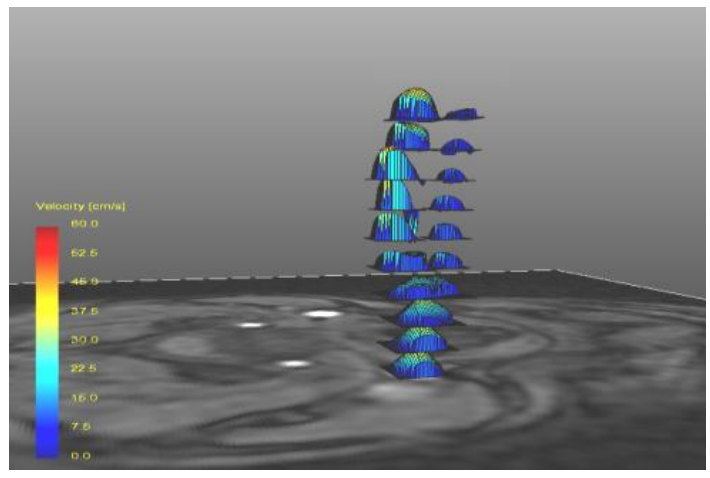

(a)

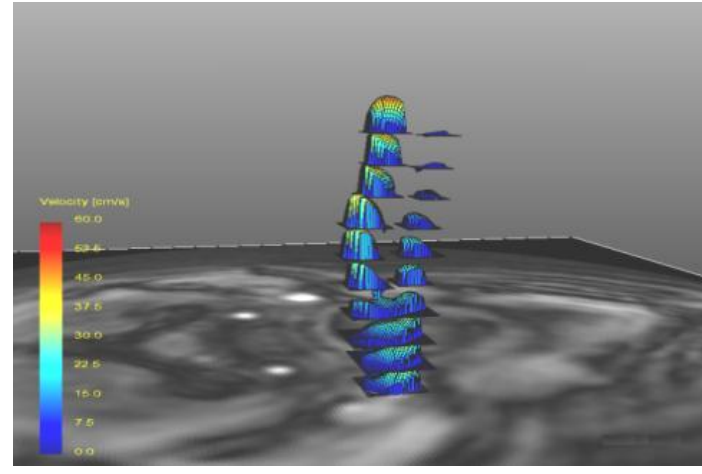

(b)

Figure 44: Velocity profile for 10 slices along right carotid artery using 4D Cartesian flow (a) and 4D spiral flow (b) in a normal volunteer during systolic phase of the cardiac cycle.

The Bland-Altman plot reveals a reasonable accuracy for 4D spiral flow with mean difference and confidence range in right CCA as 0.02 and [-1.84,2.12] and for left CCA as 0.01 and $[-1.04,0.78]$.

Figure 44 shows blood velocity profiles in 10 slices along the right carotid artery in the systolic cardiac phase in a normal volunteer. The color-coded velocity and shape of velocity profile in 4D spiral flow reveals good agreement with conventional 4D flow. Figure 45 displays zoomed flow pathlines in carotid bifurcation, right ICA, and ECA. Good correspondence between flow pathlines from 4D spiral and 4D Cartesian flow can be

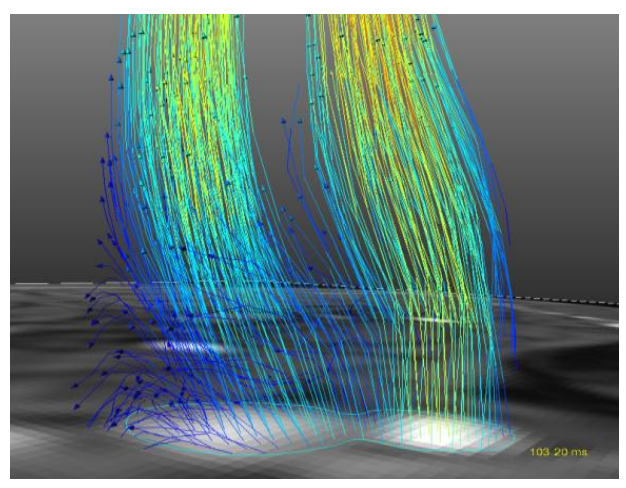

(a)

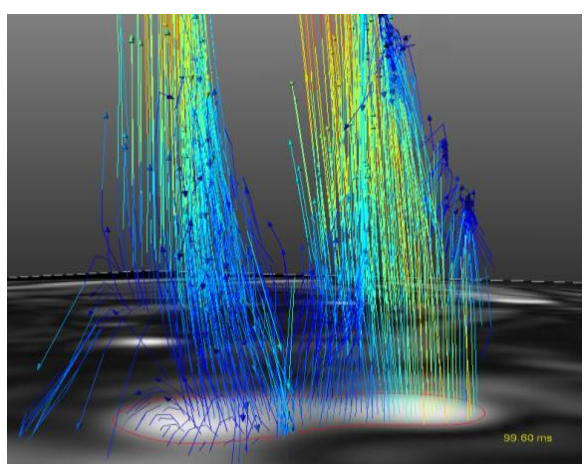

(b)

Figure 45: Flow pathlines systolic cardiac phase in right carotid artery acquired using (a) conventional 4D Cartesian PC MRI and (b) 4D spiral PC MRI. 
observed. 4D spiral flow shows slight erroneous pathlines in the ICA. 


\section{4D UTE FLOW: A PHASE-CONTRAST MRI TECHNIQUE FOR ASSESSMENT OF STENOTIC FLOWS}

\subsection{Introduction}

As mentioned earlier, conventional flow MRI based on Phase Contrast (PC) techniques are hampered due to the presence of atherosclerotic disease and vascular stenosis $[9,166-$ 168] which lead to intravoxel dephasing secondary to disturbed blood flow, and turbulence distal to narrowings $[47,114,115]$. Significant physiologic velocities can be observed in the setting of valvular disease or the coarctation of the aorta. For example, in the case of aortic stenosis (AS), velocities as high as 4-6 m/s may be observed. Such high velocities may lead to intravoxel dephasing and inaccurate flow quantification using conventional PC MRI techniques. Disturbed, swirling, and turbulent and chaotic flows may also be observed at bifurcations, branch points, and other regions of the arterial tree where blood flow is altered. In turn, these areas are more prone to atherosclerosis and narrowing in vessels. Disturbed and turbulent flow cause complex flow patterns that result in signal loss in the magnitude image and intravoxel dephasing in the phase velocity map. In previous studies, many strategies have been developed for reducing the effect of intravoxel dephasing and signal loss [4-7]. These include: accounting for higher-order motion, e.g., acceleration in velocity encoding, alignment of slice orientation to be perpendicular to the jet flow, prescribing the velocity encoding (Venc) parameter to be greater than the peak flow velocity, and improving 
the spatial resolution to mitigate partial volume errors in the setting of large velocity variations within a voxel. However, a reliable flow measurement technique in the presence of turbulence has remained elusive.

Among all the presented solutions, shortening the Echo time (TE) has been shown to have the most crucial impact in decreasing the effect of turbulent fluctuations, intravoxel dephasing, and the subsequent signal loss $[8,9,11]$. Shorter TE is effective for more accurate flow assessment because it reduces the intravoxel dephasing associated with random motions occurring in turbulent and swirling flows. O’Brien et al. [9] investigated the effect of short TE on accuracy of flow assessment in a high velocity stenotic phantom and showed that a shorter TE improves the flow measurement with a conventional $2 \mathrm{D}$ phase-contrast sequence. In addition, O’Brien et al. presented a new 2D PC sequence based on the Ultrashort TE (UTE) technique which they utilized for quantification of flow in the through-pane direction with TE reduced to $0.65 \mathrm{~ms}$ [12]. The proposed approach proved to be a more reliable technique for measuring high velocities due to robustness to intravoxel dephasing and signal loss. However, this technique had some disadvantages including underestimation of low flow rates, interdependence of slice thickness and velocity encoding parameter, and flow assessment only in the through-plane direction.

Previously, we proposed a 3D UTE-PC MRI technique benefiting from short TE to investigate the possible improvement in flow assessment in disturbed blood flow in throughplane and in-plane directions. The UTE technique in general suffers from phase errors due hardware imperfections - specifically gradient channel delays. A phase error correction technique based on auto correlation was applied in [163] to improve the robustness of 3D UTE-PC MRI. When reducing the TE to $1 \mathrm{~ms}$, flow assessment with 3D UTE in a high 
speed flow rate of $\mathrm{Q}=300 \mathrm{ml} / \mathrm{s}$ revealed a significant improvement compared to conventional PC MRI methods [169].

4D flow MRI has been investigated in several studies for quantitative flow assessment and for visualization of complex flow patterns [111, 154-159, 170-173]. This technique provides more anatomical information and comprehensive assessment of blood flow and hemodynamics. The main advantage of $4 \mathrm{D}$ flow is that the three orthogonal components of velocity vectors in a volume and at each time frame during the cardiac cycle are obtained as part of a single scan; thereby reducing scan time and flow artifacts. This is to be compared to 3D flow MRI which requires 3 separate scans to reveal each component of 3D flow velocities. Although conventional 4D PC-MRI based on Cartesian trajectory is more scan efficient than three alternative 3D scans with separate flow encodings, it can still result in relatively long scans and is prohibitive for some clinical applications. Non-cartesian trajectories have been employed in Phase contrast to obviate the problems with Cartesian trajectory. Phase Contrast with Vastly undersampled PRojection (PC VIPR) was proposed to benefit from shorter scan time and resolve the flow related artifact [174]. However, this technique limits the in-plane resolution due to isotropic resolution and dependency of inplane and through-plane resolution. A hybrid radial-cartesian strategy was developed for more efficient sampling and benefiting from isotropic in-plane resolution and stack of star approach in the through-plane [156]. Despite being highly effective in a variety of clinical applications, the TE in these techniques is still not short enough to resolve the intravoxel dephasing encountered in stenotic flows.

In this Chapter, a new 4D UTE flow MRI technique is investigated to be evaluated in steady and pulsatile stenotic phantom studies. At the fundamental level, the method 
combines the UTE technique with 4D flow to not only achieve short TE's, but to also perform velocity encoding in 3 different directions in subsequent TR's as part of a 3D acquisition. The technique permits assessment and visualization of complex flows in a 3D volume, with more accurate flow quantification and visualization due to reduced flow-related artifacts.

\subsection{Methods}

\subsubsection{Pulse sequence}

Conventional 4D flow MRI is based on application of flow sensitive bipolar gradients in three directions as part of the same scan. In a 4-point balanced (Hadamard) technique, the flow sensitive gradients are consecutively applied in three flow directions followed by a referenced flow compensated scan [175]. By subtracting the phase images of the each flow sensitive scan from the phase of the referenced flow compensated scan, the $4 \mathrm{D}$ flow data are obtained. Figure 1(a) and 1(b) demonstrate 4D Cartesian k-space data collection based on 4point balanced acquisition technique. In this sequence (Figure 1(b)), Echo Time (TE) is defined as the distance between the center of the RF pulse, to the center of the readout window. Several gradients such as slice select, bipolar, and phase encoding need to be applied during TE which results in long TE in conventional 4D flow MRI. 
(a)

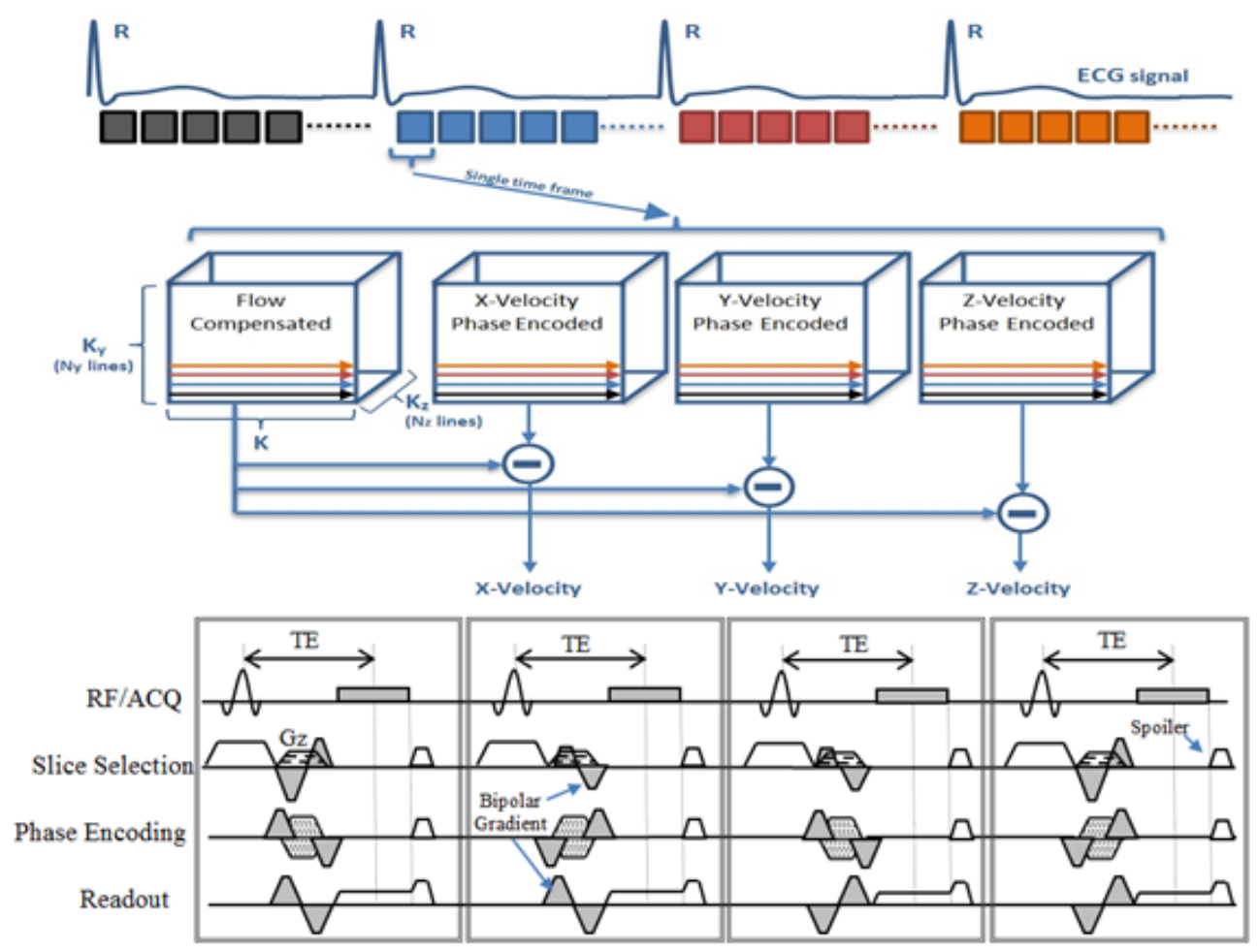

(b)

(c)

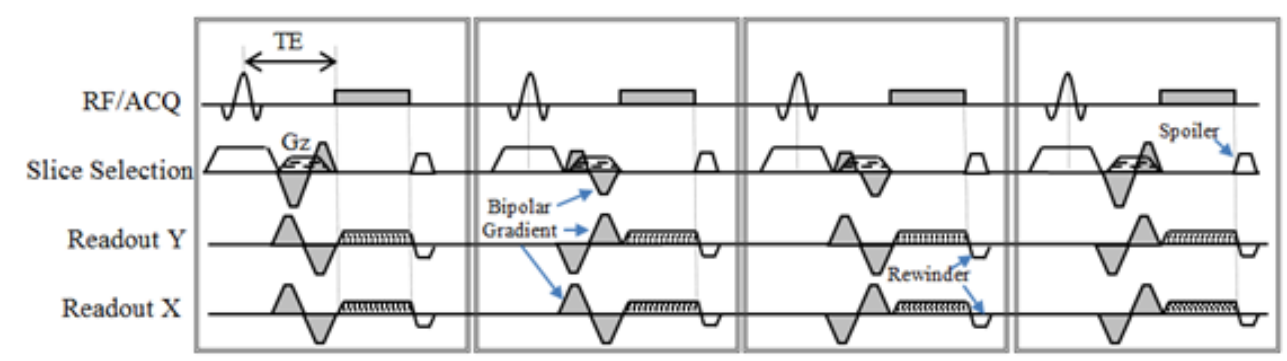

Figure 46: Timing diagram of 4D flow MRI based on 4-point balanced scheme (a). Schematic for conventional 4D flow MRI (b) and 4D UTE flow MRI sequence (c). In both techniques, four consecutive kspace lines per cardiac frame including three flow sensitive in three flow directions and one reference frame are acquired. Gray shaded gradients indicate the flow sensitive gradients in each flow direction. Subtracting

the phase of reference image from each of flow sensitive scans results in flow measurement in three directions.

UTE technique has several characteristics which help reduce the TE. In general, UTE is a center-out radial acquisition in which the k-space lines start from center of $\mathrm{k}$-space and end on a circle in k-space. Therefore, the phase encoding gradient (due to radial acquisition) and the rephasing part of readout gradient (due to center-out readout) are no longer needed. In addition, FID sampling may be started from beginning of readout gradient ramp using radial 
sampling of k-space. As a result, TE is defined as the distance between the center of the RF pulse to the beginning of the readout gradient (shown in Figure 46 (c)). The gradients which need to be applied during TE are the bipolar gradient, the slice-select gradient, as well as the phase encoding gradient in $\mathrm{Z}$ direction. To further shorten the TE, the refocusing gradient is combined with the bipolar gradient. Furthermore, the k-space volume is acquired based on the stack of stars [84] strategy which collects multiple slices in a cylindrical volume with radius $K \max$ determined by the required in-plane spatial resolution. The main advantage of stack of stars is that in contrast to kooshball [83], high in-plane resolution can be achieved without increasing the volume coverage. Compared to conventional 4D Flow MRI, 4D UTE flow MRI benefits from reduced susceptibility artifacts as a result of radial acquisition [68].Other benefit of radial acquisition is less susceptibility to motion and ghosting artifact in the phase-encode direction. Figure 46 (c) demonstrates the 4-D UTE flow sequence where for each time frame, three separate flow encoded scans (each with flow encoding only in one of $\mathrm{x}, \mathrm{y}$, and $\mathrm{z}$ directions) and one flow compensated scan are acquired. As illustrated in Figure 46 (a), the flow volumes are acquired by subtraction of each flow encoded volume in $\mathrm{x}, \mathrm{y}$, and $\mathrm{z}$ from the flow compensated volume.

\subsubsection{Experimental Setup}

An idealized, rigid phantom model of vascular occlusion with an axisymmetric Gaussian shape was machined from transparent acrylic in an otherwise straight hollow tube, also made from acrylic (33). The internal diameter of the tube was $25.4 \mathrm{~mm}$ at the inlet which narrows down to $9.04 \mathrm{~mm}$ at the throat. An occlusion of $90 \%$ area narrowing at the throat was initially aimed. However, later, the exact dimensions were measured with high resolution CT $\left(0.22 \times 0.22 \times 0.625 \mathrm{~mm}^{3}\right)$ and the area at the narrowing was determined to be $87 \%$ of the 
area at the inlet. There were additional imperfections in the fabrication process which caused the phantom to not be entirely axi-symmetric. Figure 28 shows the stenotic phantom setup

Table 9: Peak flow rates, Reynolds numbers at inlet and throat of the stenosis and other scan parameters for pulsatile and steady flow regimes as well as scan times for conventional 4D flow and 4D UTE flow MR imaging of pulsatile flows. For 4D UTE sequence, 50\% radial sampling rate was used. The scan times for steady flows were reported in Table 10. The Shelley pump was used.

\begin{tabular}{|c|c|c|c|c|c|c|c|}
\hline $\mathrm{Q}_{\max }(\mathrm{ml} / \mathrm{s})$ & & 14 & 39 & 50 & 150 & 250 & 300 \\
\hline \multirow{2}{*}{$\begin{array}{c}\text { Mean Reynolds } \\
\text { number for } \\
\text { pulsatile flow }\end{array}$} & Inlet & 53 & 150 & 190 & 570 & 950 & 1140 \\
\hline & Throat & 146 & 420 & 520 & 1570 & 2620 & 3150 \\
\hline \multirow{2}{*}{$\begin{array}{l}\text { Reynolds number } \\
\text { for steady flow }\end{array}$} & Inlet & 163 & 482 & 618 & 1854 & 3089 & 3707 \\
\hline & Throat & 452 & 1335 & 1711 & 5134 & 8557 & 10269 \\
\hline $\begin{array}{c}\text { Venc } \\
(\mathrm{cm} / \mathrm{s})\end{array}$ & & 50 & 100 & 120 & 400 & 600 & 700 \\
\hline \multirow{3}{*}{$\begin{array}{c}\text { Conventional 4D } \\
\text { flow }\end{array}$} & $\mathrm{TE}$ & 5.9 & 5.2 & 5.2 & 3.9 & 3.9 & 3.9 \\
\hline & TR & 13 & 10 & 10 & 8 & 8 & 8 \\
\hline & $\begin{array}{l}\text { Scan time for } \\
\text { pulsatile } \\
\text { (minute) }\end{array}$ & $17: 54$ & $17: 54$ & $13: 26$ & $6: 38$ & $6: 38$ & $6: 38$ \\
\hline \multirow[b]{3}{*}{ 4D UTE flow } & $\mathrm{TE}$ & 3.3 & 2.4 & 1.7 & 1.14 & 1.01 & 0.98 \\
\hline & TR & 13 & 10 & 10 & 8 & 8 & 8 \\
\hline & $\begin{array}{l}\text { Scan time for } \\
\text { pulsatile } \\
\text { (minute) }\end{array}$ & $18: 02$ & 18:02 & $13: 38$ & $6: 50$ & $6: 50$ & $6: 50$ \\
\hline
\end{tabular}

in a closed loop flow system. A CardioFlow 1000 programmable pump (Shelley Medical Imaging Technologies, London, Ontario, Canada) was used for driving steady and pulsatile flows through the flow circuit with a fluid whose viscosity was 0.0043 Pa.s and whose density was $1060 \mathrm{~kg} / \mathrm{m}^{3}$ at $18{ }^{\circ} \mathrm{C}$. To ensure fully developed laminar flow, devoid of disturbance, at the site of narrowing, a75-cm long straight rigid acrylic tube was positioned 
upstream of the narrowing. In addition to the Shelley pump, a pump produced by LB Engineering (Berlin, Germany) was used for further validations of the proposed method.

\subsubsection{Flow regimes}

Various steady and pulsatile flow rates representing low, medium, and high flow rate corresponding to a range of Reynolds numbers were examined using the aforementioned experimental setup. Reynolds number can be defined as

$$
\operatorname{Re}=\frac{\rho v D}{\mu}
$$

where $\rho$ is the density of the fluid $\left(\mathrm{kg} / \mathrm{m}^{3}\right), \mathrm{v}$ is the mean velocity of the object relative to the fluid (m/s), D is the diameter of the tube (m), and $\mu$ is the dynamic viscosity of the fluid $(\mathrm{kg} /(\mathrm{m} \cdot \mathrm{s}))$. The Re number at the throat of narrowing can be calculated using

$$
\frac{\mathrm{Re}_{2}}{\mathrm{Re}_{1}}=\sqrt{\frac{\mathrm{A}_{1}}{\mathrm{~A}_{2}}}
$$

where $A_{1}$ and $A_{2}$ are the area of phantom at the inlet and at the throat of narrowing, respectively. Table 9 reports how the flow rates translate to the Reynolds numbers at the inlet and at the throat of narrowing for steady and pulsatile flow regimes. In Table 9, the

pulsatile Reynolds numbers were calculated based on the peak flow rates. The mean Reynolds numbers for pulsatile flow is approximately one third of peak Reynolds numbers. For instance, the mean Reynolds number at $300 \mathrm{ml} / \mathrm{s}$ pulsatile flow rate at inlet and throat of narrowing are 1140 and 3150 respectively. Any Reynolds number greater than 2,000 is associated with the turbulent flow regime. 


\subsubsection{Imaging protocol}

Imaging was performed on a Philips Achieva 1.5T scanner (Philips Healthcare, Best, NL) using a 16-element SENSE knee coil. The gradient strength of $21 \mathrm{mT} / \mathrm{m}$ and a slew rate of $100 \mathrm{~T} / \mathrm{m} / \mathrm{s}$ were used for both conventional 4D PC and 4D UTE PC sequences. These are the values for conventional clinical acquisitions. Though the scanner is capable of achieving a higher gradient strength and slew rate (on Philips Achieva 1.5T scanner these are 33 $\mathrm{mT} / \mathrm{m}, 180 \mathrm{~T} / \mathrm{m} / \mathrm{s}$ ), the aforementioned values strike a balance between accurate flow measurement and eddy current induced phase errors due to fast gradient switching. The imaging volume covered $60 \mathrm{~mm}$ of phantom including $\sim 10 \mathrm{~mm}$ proximal (3 slices) and $\sim 36$ $\mathrm{mm}$ distal (12 slices) to the center of the narrowing (Figure 47). The throat of narrowing, which included 2 axial slices, was positioned at the iso-center of magnet as well as at the center of the SENSE knee coil. Three distal slices were located along the part of the phantom with gradual narrowing from the throat to the part of tube with constant diameter which were not considered as being part of proximal, throat, or the distal slices in the

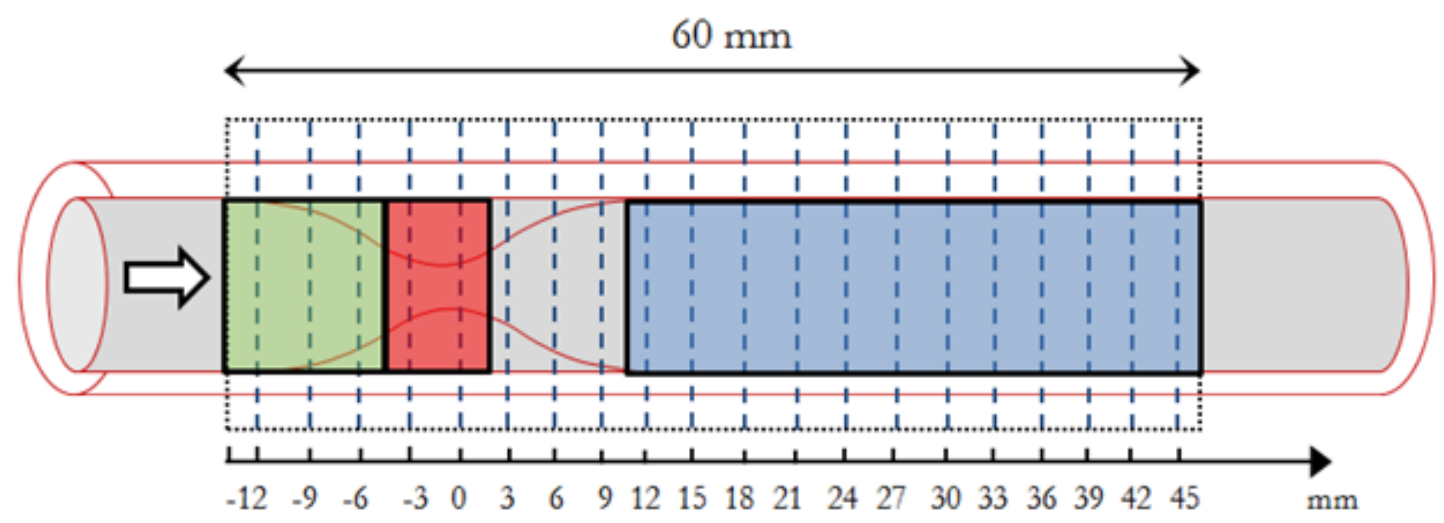

Figure 47: A schematic geometry of the phantom and the sagittal extent of the FOV for data collection.

Dashed lines represent the location of axial slices along the phantom. The green, red, and blue areas are regions proximal, at the throat, and distal to the stenosis that were considered separately in the data analyses. 
assessments.

Two sequences were used: conventional 4D flow MRI based on Cartesian trajectory and 4D UTE flow MRI based on the center-our radial trajectory. The common scan parameters for two sequences were $\mathrm{FOV}=100 \times 100 \times 60 \mathrm{~mm}$, flip angle $=10$ degrees, and spatial resolution $=1.5 \times 1.5 \times 3.0 \mathrm{~mm}$ (leading to 20 axial slices). For pulsatile flow regimes, an ECG trigger signal was sent to the scanner with $\sim 1 \mathrm{~Hz}$ frequency and 14 frames in each cycle were collected based on prospective ECG triggered scheme. For the 4D UTE sequence, four radial sampling rates $(25 \%, 50 \%, 75 \%$, and $100 \%$ for full $\mathrm{k}$-space acquisition) were studied. Note that $100 \%$ sampling refers to a full k-space acquisition with no under-sampling. For the aforementioned parameters, the number of radial spokes for each slice at $100 \%$ sampling rate was 1728 and this number was reduced to 432 at 25\% sampling density. One may note that, the under-sampling of radial k-space lines mainly affects the off-center of k-space since an over-sampling already exists when adopting radial sampling. A stack of stars trajectory was used to collect the 3D volume with the UTE scheme. Table 9 shows the flow rates with corresponding Reynolds numbers which were utilized in this study to investigate imaging of steady flows as well as imaging of low, medium, and high physiologic flows encountered invivo. The Venc, TE, TR, and scan times for each flow regime may also be found in Table 9. The parameters reported in this Table are for the 4D UTE flow sequence with $50 \%$ radial sampling. Additionally, please note that the Venc parameter was the same for all three directions.

\subsubsection{Phase Corrections and ROI Definitions}

To correct the phase of flow-on experiments for all 4D flow acquisitions (conventional and UTE), the phase image of flow-off experiments with identical imaging parameters were 
subtracted from the phase image of the flow-on experiments. In addition, for all 4D UTE acquisitions including flow-on and flow-off scans, phase errors due to gradient delay and hardware imperfections were also corrected based on an auto correlation technique which was employed to measure the k-space trajectory delays [163]. The measured trajectory delays were used to correct the trajectory delay offset in all subsequent scans with identical parameters. It should be mentioned that the trajectory delay for pulsatile and steady flow regimes at each flow rate is identical since gradient waveforms for steady and pulsatile flow regimes are themselves identical.

To compute flow waveforms from MRI velocity data, the Region of Interest (ROI) in each frame at each slice was manually selected based on a circular mask with the known diameter of the phantom. The radius of circle was kept constant throughout the cardiac cycle and at different slice locations except the slices in the vicinity of stenosis throat where the diameter of the tube varies. Since flow at the boundary is small, partial volume effects in calculating flow waveforms are thought to be of little concern in calculating the flow rates. Nevertheless, to reduce the effect of partial volume, pixels having less than $50 \%$ area inside the ROI were excluded from the analysis. Calculation of flow rate for a specific slice in the phantom and for a specific time point, simply involved summing up the through-plane encoded velocities inside the corresponding ROI. In order to compare the measured flow using conventional and the proposed 4D flow technique with the prescribed flow at the pump, the Relative Root Mean Square Error (RRMSE) metric was adopted

$$
\operatorname{RRMSE}=100 \% \times \sqrt{\frac{\sum_{n} \sum_{t}\left(\mathrm{Q}_{\mathrm{UTE}} / \mathrm{conv}(\mathrm{t})-\mathrm{Q}_{\mathrm{pre}}(\mathrm{t})\right)^{2}}{\sum_{n} \sum_{t}\left(\mathrm{Q}_{\mathrm{pre}}(t)\right)^{2}}}
$$


where $Q_{\text {pre }}$ represents the prescribed flow at the pump and $Q_{U T E / c o n v}$ represents the measured flow using 4D UTE flow MRI or Conventional 4D flow MRI. $n$ and $t$ denote slice positions along the length of the phantom and the number of cardiac phases, respectively. For example, $n=12$ for the number of slices considered distal to the stenosis. For steady flows, $t$ is equal to one whereas for pulsatile flows $t$ is the number of cardiac phases.

All the post-processing and the flow assessments were performed using Matlab software (The Mathworks, Natick, MA).

\subsection{Results}

\subsubsection{Number of projections and its effect on scan time and accuracy}

The scan time for the 4D UTE flow is longer than conventional 4D flow. This is due to the difference in the k-space trajectories between the two methods. For example, to collect an $\mathrm{N} \times \mathrm{N}$ image, with the Cartesian scheme, $\mathrm{N}$ k-space lines are sufficient. However, to collect an $\mathrm{N} \times \mathrm{N}$ image, with radial acquisitions, $\pi \mathrm{N}$ k-space lines are required. Therefore, in adopting the UTE scheme, it is critical to reduce the scan time, especially for in-vivo studies. One approach to reduction of the scan time as part of radial acquisitions is to under-sample the $\mathrm{k}$-space in the azimuthal direction. This obviates the need to apply fast imaging techniques such as parallel imaging or compressive sampling. Under-sampling in the phaseencode direction as part of a Cartesian acquisition causes wrapping artifact in phase-encode

direction. In radial- k-space acquisitions however, under-sampling produces streaking artifacts. 
In this study, the effect of radial sampling has been examined in the stenotic phantom for various steady flow rates and in particular, its effect on the total scan time and flow measurement accuracy has been determined.

As may be seen in Table 10 , the $25 \%$ radial sampling rate resulted in the shortest scan time particularly at high flow rates. However, at this sampling rate, the measured flow and RRMSE revealed considerable inaccuracies. 50\% sampling rate resulted in the same scan time as the conventional 4D flow sequence while the flow measurement accuracy was found to be acceptable when compared to flow prescribed at the pump (this should be viewed in light of the fact that according to pump's manufacturer, there can be up to a $\pm 3 \%$ error

Table 10: Steady flow measurement averaged for 12 axial slices distal to throat of the stenosis using conventional 4D flow MRI and 4D UTE flow MRI with various sampling rates and at different flow rates.

Table entries are mean \pm standard deviation (across the slices). The Shelley pump was used.

\begin{tabular}{|c|c|c|c|c|c|}
\hline $\begin{array}{l}\text { Prescribed Flow } \\
\text { rates }(\mathrm{ml} / \mathrm{s})\end{array}$ & & 14 & 50 & 150 & 300 \\
\hline \multirow{3}{*}{$\begin{array}{l}\text { 4D UTE with } 25 \% \\
\text { sampling }\end{array}$} & $\begin{array}{c}\text { Measured Flow } \\
(\mathrm{ml} / \mathrm{s})\end{array}$ & $10.11 \pm 2.89$ & $37.65 \pm 3.99$ & $118.55 \pm 14.62$ & $232.53 \pm 16.23$ \\
\hline & Scan time [84] & 28.8 & 18.5 & 12.2 & 11.4 \\
\hline & RRMSE (\%) & 25.26 & 24.70 & 20.89 & 22.41 \\
\hline \multirow{3}{*}{$\begin{array}{l}\text { 4D UTE with } 50 \% \\
\text { sampling }\end{array}$} & $\begin{array}{c}\text { Measured Flow } \\
(\mathrm{ml} / \mathrm{s})\end{array}$ & $12.16 \pm 2.06$ & $43.35 \pm 5.36$ & $129.29 \pm 15.53$ & $258.82 \pm 16.41$ \\
\hline & Scan time (s) & 56.1 & 35.5 & 24.3 & 22.5 \\
\hline & RRMSE (\%) & 13.33 & 12.96 & 12.54 & 13.72 \\
\hline \multirow{3}{*}{$\begin{array}{l}\text { 4D UTE with } 75 \% \\
\text { sampling }\end{array}$} & $\begin{array}{c}\text { Measured Flow } \\
(\mathrm{ml} / \mathrm{s})\end{array}$ & $13.25 \pm 1.79$ & $47.52 \pm 4.53$ & $137.44 \pm 10.97$ & $272.02 \pm 14.54$ \\
\hline & Scan time (s) & 83 & 54 & 35.1 & 32.4 \\
\hline & RRMSE (\%) & 5.43 & 5.24 & 7.41 & 9.32 \\
\hline \multirow{3}{*}{$\begin{array}{l}\text { 4D UTE } \\
\text { with } 100 \% \\
\text { sampling }\end{array}$} & $\begin{array}{c}\text { Measured Flow } \\
(\mathrm{ml} / \mathrm{s})\end{array}$ & $16.42 \pm 1.34$ & $55.76 \pm 5.21$ & $149.49 \pm 8.47$ & $297.73 \pm 15.80$ \\
\hline & Scan time (s) & 110 & 71 & 47.2 & 43.4 \\
\hline & RRMSE (\%) & 16.46 & 11.52 & 1.54 & 1.75 \\
\hline \multirow{3}{*}{$\begin{array}{c}\text { Conventional 4D } \\
\text { flow }\end{array}$} & $\begin{array}{c}\text { Measured Flow } \\
(\mathrm{ml} / \mathrm{s})\end{array}$ & $13.17 \pm 1.94$ & $52.89 \pm 5.27$ & $131.19 \pm 17.65$ & $205.34 \pm 45.81$ \\
\hline & Scan time (s) & 33.9 & 31.5 & 30.1 & 29.7 \\
\hline & RRMSE (\%) & 5.93 & 5.78 & 8.51 & 31.44 \\
\hline
\end{tabular}


between prescribed and measured flow). Relative to the prescribed flow, 4D UTE flow measurement with $75 \% \mathrm{k}$-space sampling resulted in accurate flow measurement for both the low and the high flow rates while in comparison to $100 \%$ sampling resulted in reduced total scan time. At low flow rates, the measured flow rates with conventional 4D flow are in excellent agreement with the prescribed flow at the pump. However, at high flow rates, a significant difference can be seen between the conventional 4D flow and the prescribed flow with an RRMSE exceeding 30\%. This difference is due to flow related artifacts observed in the conventional sequence.

\subsubsection{Steady flows}

Linear regression analysis was used for assessment of flow at three locations: $12 \mathrm{~mm}$ proximal to the stenosis, at the throat, and $21 \mathrm{~mm}$ distal to the stenosis. Results are shown in Figure 48. The flow measurements made both proximal to the stenosis and at the throat of the stenosis using conventional and UTE techniques show a very good agreement with the prescribed flow at the pump. However, distal to the stenosis, while the UTE technique has excellent accuracy, the intercept and slope values calculated using the conventional sequence point to large errors.

To demonstrate the effect of phase error corrections performed on UTE and conventional results, Figure 49 displays measured flow at each slice along the phantom for the case of $Q=150 \mathrm{ml} / \mathrm{s}$ steady flow rate. Similar investigations were also performed for other flow rates which were similar. The red and blue plots display flow rates as a function of position along the length of the phantom for each of the 4D conventional and 4D UTE scans. It is evident that after $\sim 27 \mathrm{~mm}$ distal to the throat of the stenosis, the flow rate obtained by the UTE sequence develops significant errors. This is mainly due to 

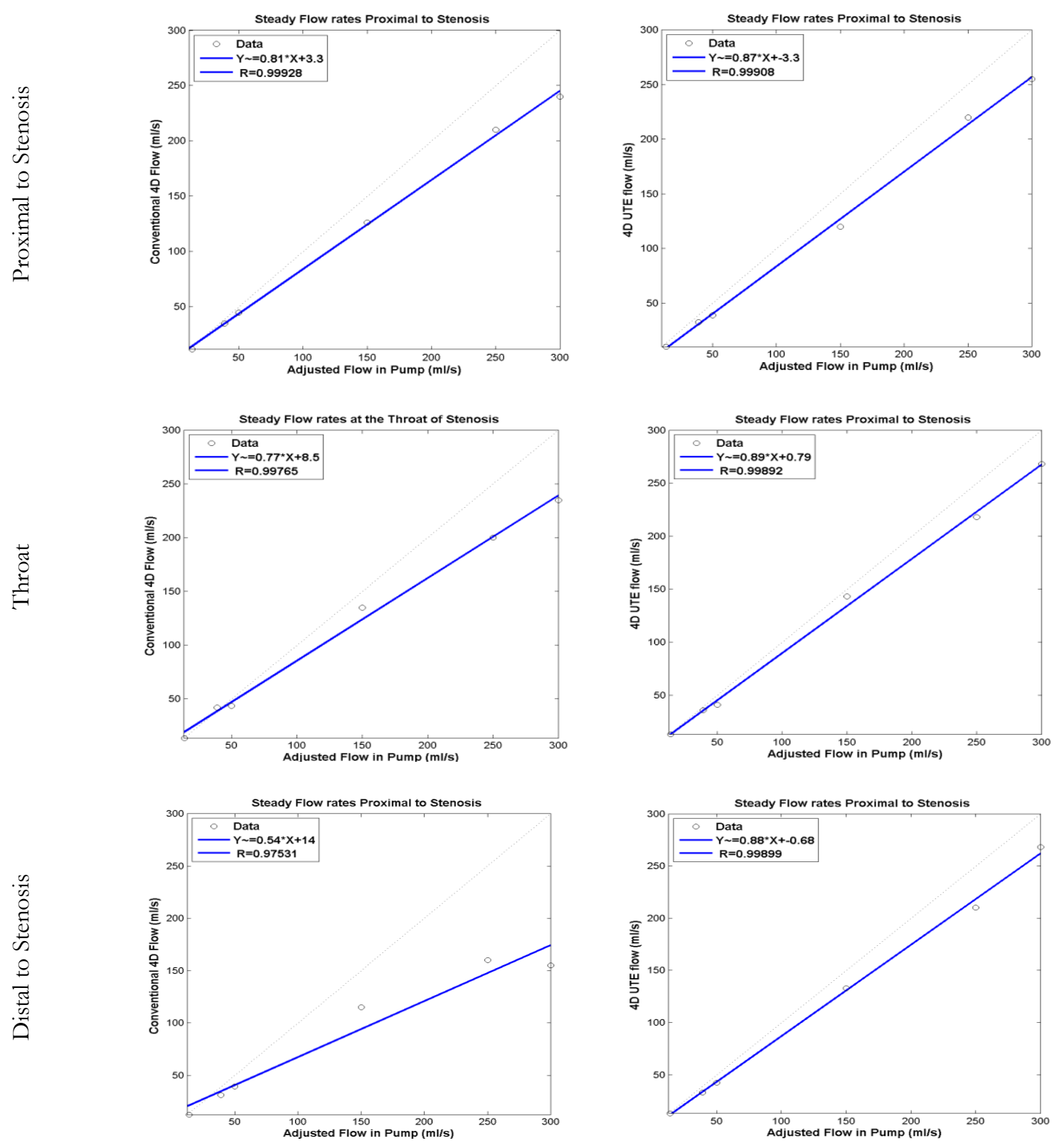

(a)

(b)

Figure 48: Linear regression analysis at three locations proximal (first row), at the throat (second row), and distal (third row) to stenosis using conventional 4D flow MRI (a) and 4D UTE flow MRI (b). The x-axis is the prescribed flow at the pump and y-axis the measured flow using two sequences. The Shelley pump was used. sensitivity of the UTE sequence to nonuniformity of RF pulse and consequent eddy current which results in phase offset in off-center slices. This error is negligible before $\sim 27 \mathrm{~mm}$ distal to the throat of stenosis since the throat of the stenosis was positioned at the center of 


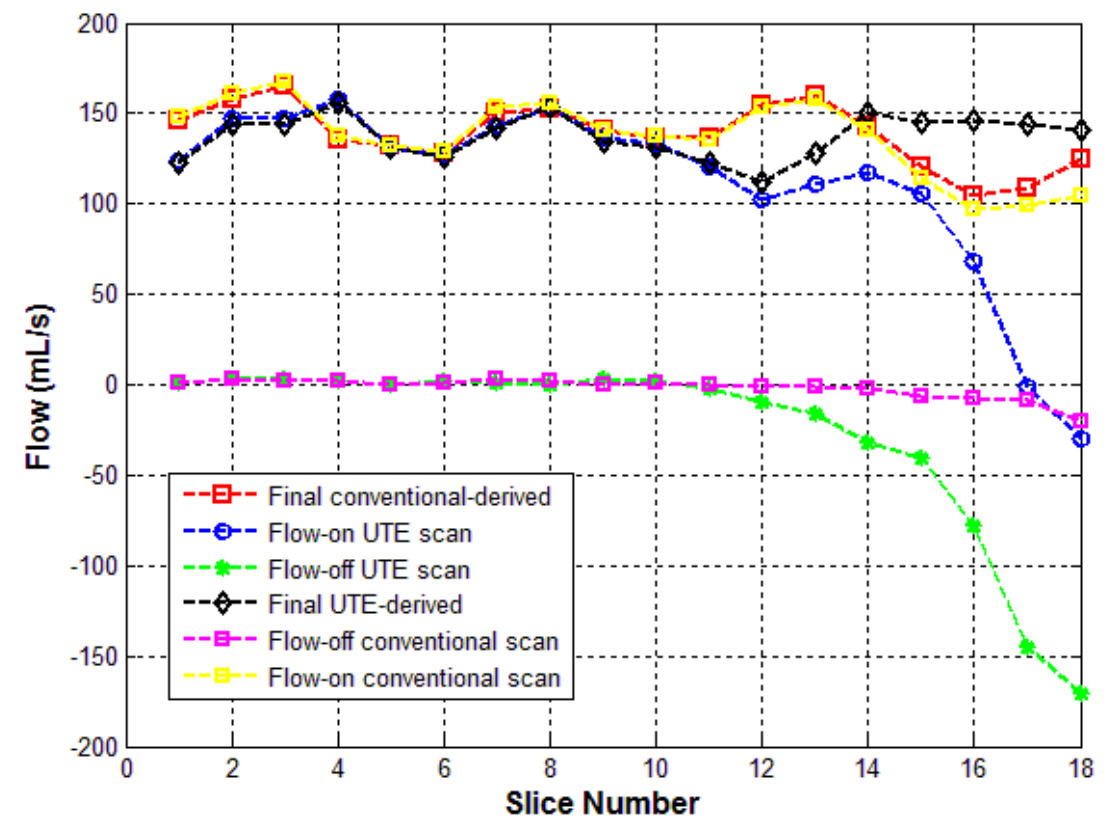

Figure 49: Measured flow at each slice along the phantom at $Q=150 \mathrm{ml} / \mathrm{s}$ steady flow. The green plot shows the measured flow for the flow-off experiment using UTE sequence and the flow value diverges away from zero towards the end of the coil. The errors for UTE-derived flow rate (blue plot) was reduced using flow-off experiment as shown by the black plot. The Shelley pump was used.

coil and the field inhomogeneity is in significant for the slices close the throat of stenosis (the FOV includes more slices distal to the center the phantom). The green plot displays the flow-off scan which was used to reduce the flow error. Ideally, without any phase error, the flow-off plot would be expected to be horizontal and around zero (similar to the yellow plot for the flow-off conventional 4D scan) but the flow-off plot points to a significant error after $\sim 27 \mathrm{~mm}$ distal to the throat of stenosis. The phase values of flow-off scan were subtracted from the phase values of UTE flow-on scan to correct the phase offset due to the field inhomogeneity. The black plot shows results from the UTE sequence after phase correction -- a good correlation between phase-corrected UTE scans and phase-corrected conventional scans is observed. 

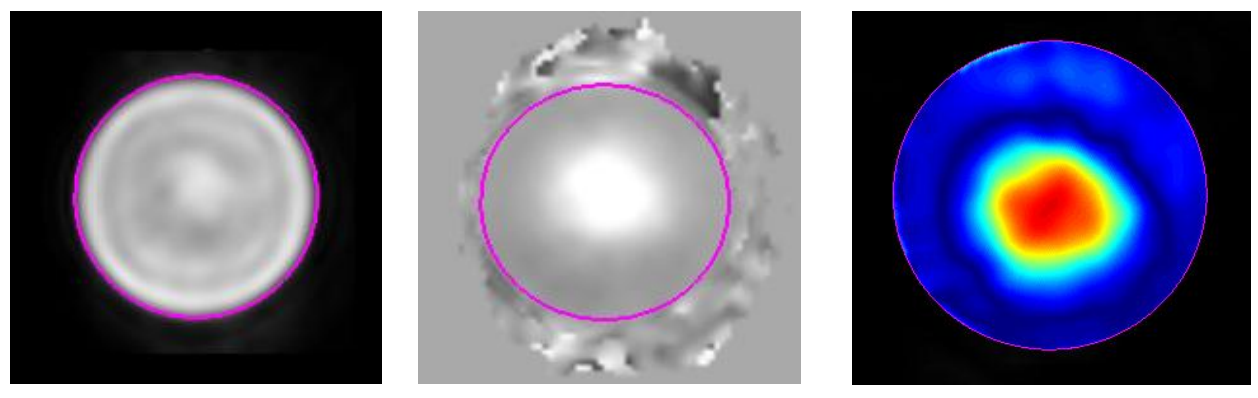

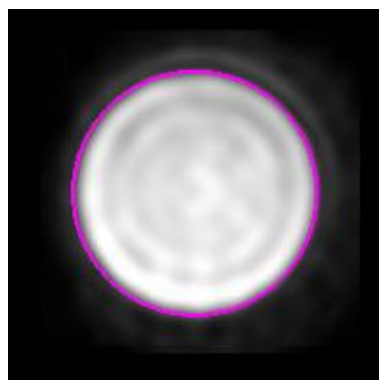

(a)

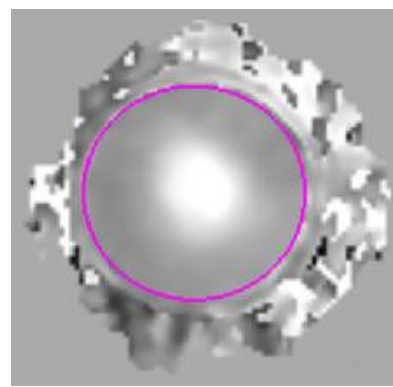

(b)

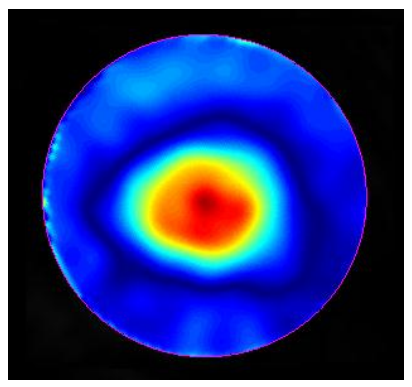

(c)

Figure 50: An axial slice $21 \mathrm{~mm}$ distal to throat of the stenosis at $\mathrm{Q}=50 \mathrm{ml} / \mathrm{s}$ steady flow rate using conventional 4D flow (top row) and 4D UTE flow (bottom row). The magnitude (a), phase (b) and velocity contour (c) indicate a good agreement between two sequences at low flow rates. The Shelley pump was used.

Figure 50 demonstrates results at a slice $21 \mathrm{~mm}$ distal to the narrowing at $Q=50 \mathrm{ml} / \mathrm{s}$ using conventional 4D flow (top row) and 4D UTE flow (bottom row). The magnitude (a), phase (b), and velocity contour (c) for these sequences reveal excellent agreement. No noticeable signal loss or intravoxel dephasing can be observed at this flow rate with either sequences. Figure 51 demonstrates a sagittal view of phantom and velocity profile (top row) and flow streamlines (bottom row) at $Q=50 \mathrm{ml} / \mathrm{s}$ flow rate at three axial slices located proximal, at the throat and distal to stenosis using conventional 4D flow MRI (a) and 4D UTE flow MRI (b). The velocity profiles at all three locations for these sequences reveal good agreement. No noticeable signal loss or intravoxel dephasing can be observed at this flow rate with either sequences. Flow streamlines were generated using a dedicated visualization software (GTFlow, GyroTools, Zurich, Switzerland). The flow streamlines at these three locations using conventional 4D flow and 4D UTE sequences show good 

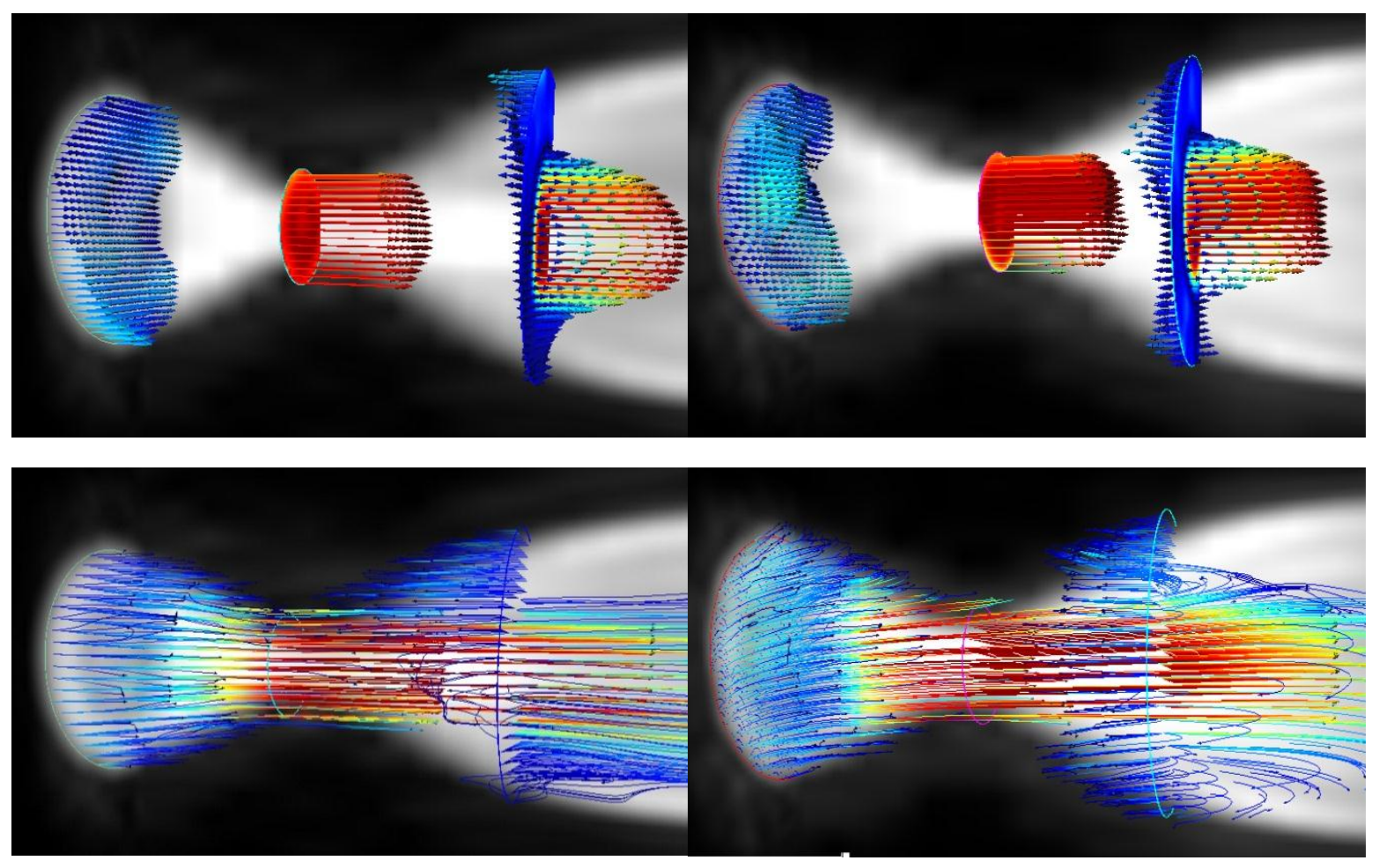

(a)

(b)

Figure 51: A sagittal view of phantom at the throat of stenosis at $Q=50 \mathrm{ml} / \mathrm{s}$ steady flow rate. The velocity profile (first row) and flow streamlines (second row) are shown using conventional 4D flow MRI (a) and 4F UTE flow MRI (b). Velocity profiles proximal, at the throat, and distal to stenosis show an excellent agreement between two sequences. Flow streamlines at three locations display good correlation in both sequences. The Shelley pump was used.

correlation. In Figure 52 the magnitude (a), phase (b), and velocity contour (c) for an axial slice $21 \mathrm{~mm}$ distal to stenosis using conventional (top row) and 4D UTE (bottom row) flow MRI are shown for the $300 \mathrm{ml} / \mathrm{s}$ steady flow rate. The conventional sequence results in a signal loss in magnitude image, intravoxel dephasing in phase image, and velocity error in velocity contour. The result from UTE sequence displays a considerable improvement in velocity contour and the signal loss while the intravoxel dephasing is entirely removed. In Figure 53, the velocity profile (top row) and flow streamlines (bottom row) are shown for an axial slice $21 \mathrm{~mm}$ distal to the center of the stenosis at $\mathrm{Q}=300 \mathrm{ml} / \mathrm{s}$ steady flow rate using conventional (a) and 4D UTE flow MRI (b). It is evident that the conventional sequence results in errors in the velocity profile and streamlines. 

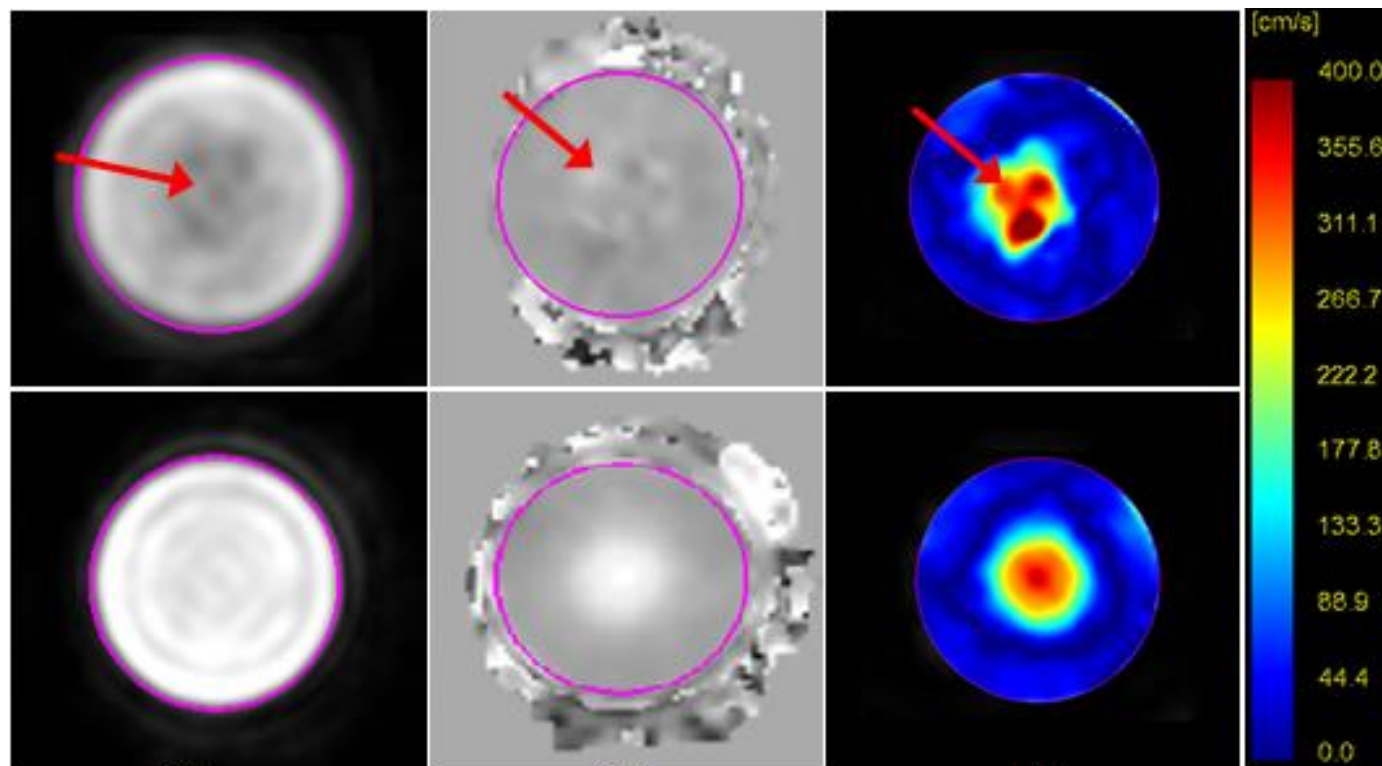

(a)

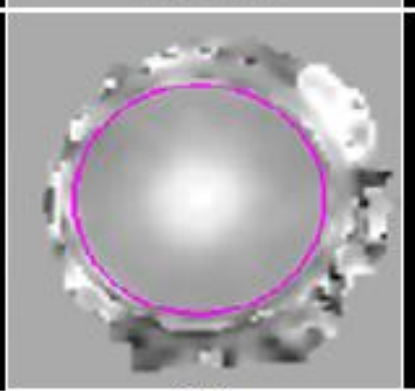

(b)

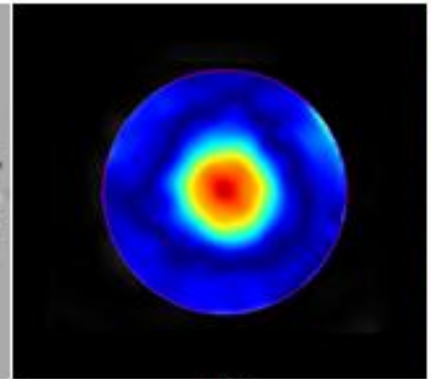

(c)

Figure 52: An axial slice $21 \mathrm{~mm}$ distal to the throat of the stenosis for the case of steady flow at $\mathrm{Q}=300$ $\mathrm{ml} / \mathrm{s}$. The magnitude (a), phase (b), and velocity contours (c) from conventional 4D flow MRI (top row) as well as 4D UTE flow MRI (bottom row) are displayed. The intravoxel dephasing and signal loss is visible in the case of long TE (top row). The removal of intravoxel dephasing and signal loss in the UTE sequence is appreciable.

The Shelley pump was used.
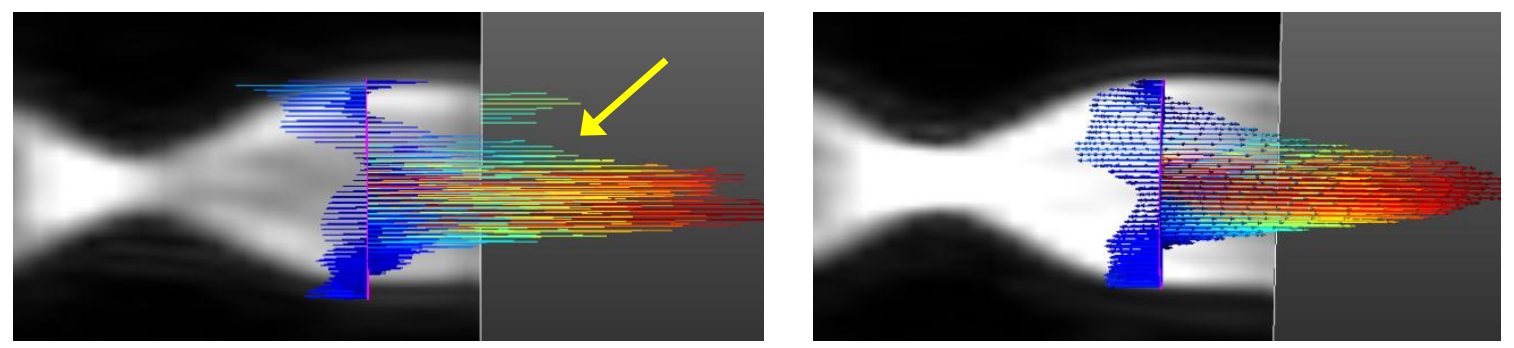

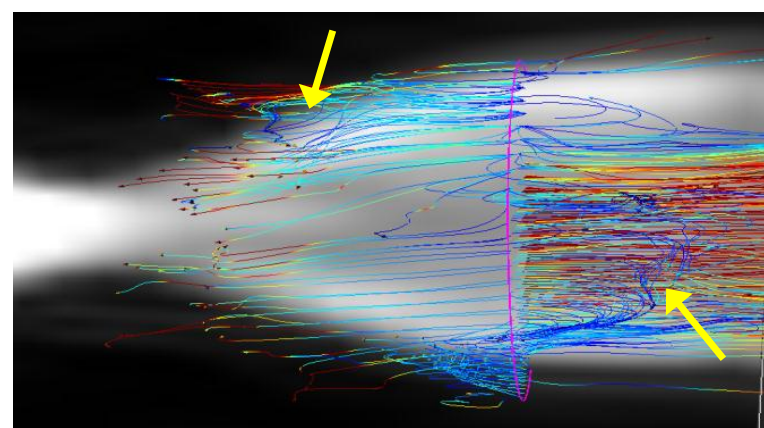

(a)

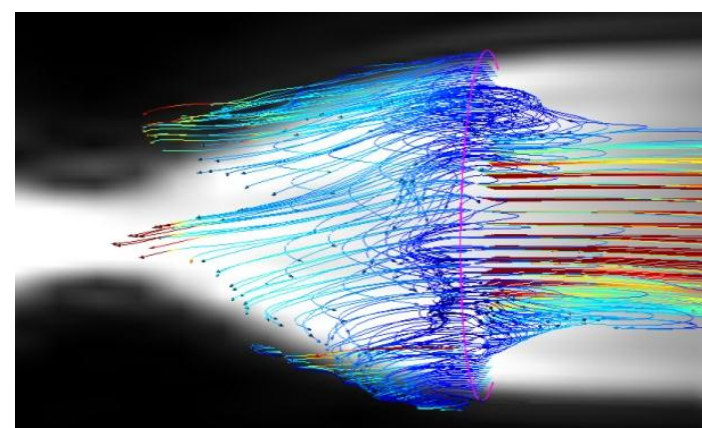

(b)

Figure 53: A sagittal view of phantom at $\mathrm{Q}=300 \mathrm{ml} / \mathrm{s}$ steady flow rate. The velocity profile (first row) and flow streamlines (second row) are shown for conventional 4D flow MRI (a) and 4D UTE flow MRI (b). The flowrelated artifact and intravoxel dephasing (yellow arrows) caused inaccurate velocity profile and streamlines in conventional sequence. The Shelley pump was used. 


\subsubsection{Pulsatile flows}

Flow assessment and analysis was also performed in the phantom with different pulsatile flow rates using both conventional 4D flow and the proposed 4D UTE flow. The same biphasic flow waveform was used in all cases (see Figure 11(a)). However, the peak flow rate at the pump, Qmax, was adjusted as shown in Table 9 to produce six distinct waveforms. To compare conventional and UTE sequences, the peak systolic flow was determined for each slice and averaged for 3 slices proximal to the stenosis and 12 slices distal to the stenosis.

Table 11: Pulsatile flow measurement averaged for 3, 2, and 12 slices located proximal, at the throat, and distal to throat of the stenosis (Figure 3) using conventional 4D flow MRI and 4D UTE flow MRI at different flow rates. Table entries for measured peak systolic flow are mean \pm standard deviation (across the slices). RRMSE is the Relative Root Mean Squared Error between prescribed pulsatile flow and the measured flow

(Eq.).

\begin{tabular}{|c|c|c|c|c|c|c|c|}
\hline \multirow{2}{*}{$\begin{array}{c}\mathbf{Q}_{\max } \\
(\mathrm{ml} / \mathbf{s})\end{array}$} & & \multicolumn{3}{|c|}{ Conventional 4D flow } & \multicolumn{3}{|c|}{ 4D UTE flow } \\
\hline & & Proximal & Throat & Distal & Proximal & Throat & Distal \\
\hline \multirow{3}{*}{14} & RRMSE (\%) & 10.4 & 8.97 & 6.87 & 13.23 & 11.33 & 9.89 \\
\hline & \multirow{2}{*}{ Peak sys flow } & $10.75 \pm$ & $10.39 \pm$ & $11.35 \pm$ & $11.87 \pm$ & $10.86 \pm$ & $10.30 \pm$ \\
\hline & & 2.54 & 2.8 & 2.1 & 2.11 & 2.74 & 2.56 \\
\hline \multirow{3}{*}{39} & RRMSE (\%) & 7.86 & 7.34 & 4.22 & 12.31 & 10.54 & 8.05 \\
\hline & \multirow{2}{*}{ Peak sys flow } & $30.43 \pm$ & $32.21 \pm$ & $33.27 \pm$ & $31.84 \pm$ & $30.40 \pm$ & $29.98 \pm$ \\
\hline & & 3.35 & 3.90 & 4.21 & 4.53 & 4.14 & 3.90 \\
\hline \multirow{3}{*}{50} & RRMSE (\%) & 10.66 & 11.75 & 11.21 & 13.34 & 14.21 & 12.54 \\
\hline & \multirow{2}{*}{ Peak sys flow } & $42.98 \pm$ & $43.74 \pm$ & $44.28 \pm$ & $41.48 \pm$ & $42.72 \pm$ & $39.21 \pm$ \\
\hline & & 4.44 & 3.28 & 3.72 & 4.2 & 4.73 & 5.53 \\
\hline \multirow{3}{*}{150} & RRMSE (\%) & 13.76 & 18.97 & 28.89 & 25.75 & 20.60 & 22.43 \\
\hline & Peol cus flow & $133.20 \pm$ & $130.83 \pm$ & $128.20 \pm$ & $129.53 \pm$ & $130.1 \pm$ & $135.4 \pm$ \\
\hline & Than sys tive & 11.76 & 18.21 & 18.57 & 8.54 & 7.93 & 7.53 \\
\hline \multirow{3}{*}{250} & RRMSE (\%) & 20.75 & 23.43 & 46.19 & 18.16 & 18.76 & 19.12 \\
\hline & Peal sus flow & $207.01 \pm$ & $192.39 \pm$ & $171.01 \pm$ & $209.48 \pm$ & $210.83 \pm$ & $221.41 \pm$ \\
\hline & The syo tiver & 15.95 & 20.10 & 23.43 & 18.85 & 14.7 & 10.4 \\
\hline \multirow{3}{*}{300} & RRMSE (\%) & 27.38 & 30.54 & 71.19 & 23.57 & 23.51 & 25.20 \\
\hline & \multirow{2}{*}{ Peak sys flow } & $230.45 \pm$ & $215.94 \pm$ & $175.10 \pm$ & $253.52 \pm$ & $251.9 \pm$ & $240.6 \pm$ \\
\hline & & 25.87 & 32.53 & 40.33 & 12.72 & 11.8 & 11.1 \\
\hline
\end{tabular}


Table 11 shows that the measured RRMSE between conventional 4D flow MRI and 4D UTE flow MRI proximal, at the throat, and distal to the stenosis at low $(14,39,50 \mathrm{ml} / \mathrm{s})$ are closely correlated. One may notice that for both sequences, RRMSE values are relatively large as the measured flows are lower than the prescribed flows at the pump. This is mainly due to compliance of tubes and connectors which has the effect of damping down the flow waveform at the inlet. At medium-high flow rate $(150 \mathrm{ml} / \mathrm{s})$, the measured RRMSE using both sequences is slightly larger, especially at the throat and distal to stenosis. The calculated RRMSE at high flow rates $(250,300 \mathrm{ml} / \mathrm{s})$ proximal to the stenosis shows acceptable errors with both sequences. This error is slightly higher at the throat of the stenosis using the conventional sequence due to appearance of turbulent jet. However, the measured flow distal to stenosis using conventional techniques results in a significant underestimation of peak systolic flow distal to the stenosis leading to a large RRMSE value. 4D UTE flow estimated the flow more accurately at high flow rates at both proximal and distal locations to the stenosis leading to acceptable RRMSE values.

Linear regression analysis was performed for assessment of accuracy at three different locations proximal, throat, and distal to the stenosis and results are shown in Figure 54. The data points represent the measured flow rate for a specific cardiac phase averaged in 3 slices proximal to stenosis, 2 slices at the throat of stenosis, and 12 slices distal to stenosis for different flow rates. The measured values from linear regression analysis reveal a relatively good agreement between conventional 4D flow MRI and 4D UTE flow MRI $\left(\mathrm{R}^{2}>0.97\right)$ proximal and at the throat of stenosis. The $\mathrm{R}$ value measured distal to the stenosis reveals a significant difference between conventional and UTE sequences $\left(R^{2}>0.90\right)$ - this is mainly due to underestimation of measured flow when using the conventional technique. Figure 55 


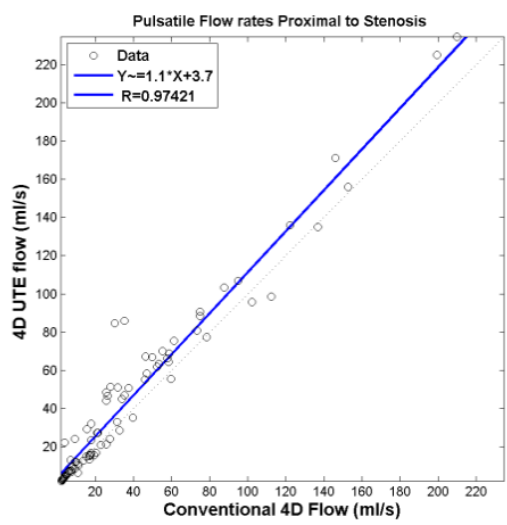

(a)

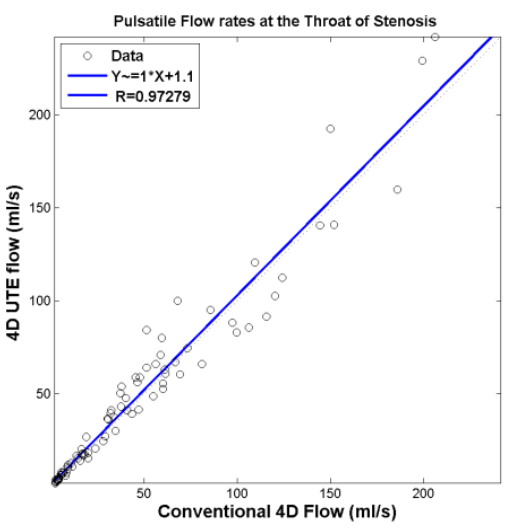

(b)

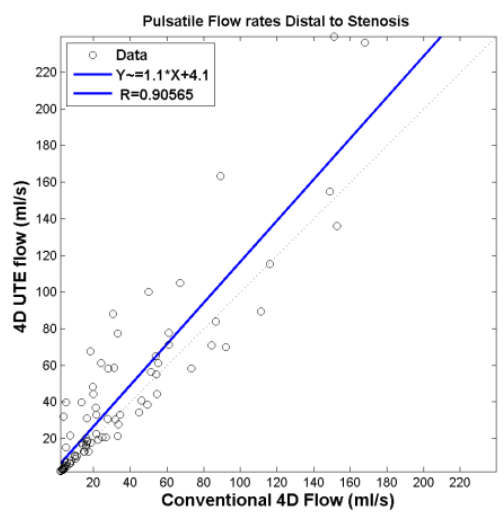

(c)

Figure 54: Linear regression analysis for measured pulsatile flow rates between conventional 4D flow MRI and 4D UTE flow MRI at three locations: proximal (a), throat (b), and distal (c) to the stenosis. Each data point represents the measured flow rate for a specific cardiac phase averaged in 3, 2, and 12 slices proximal, throat, and distal to stenosis. The data points show more difference in flow measurement at high flow rates. The measurement at the throat of the stenosis has the highest correlation between two techniques at all flow rates.

The Shelley pump was used.
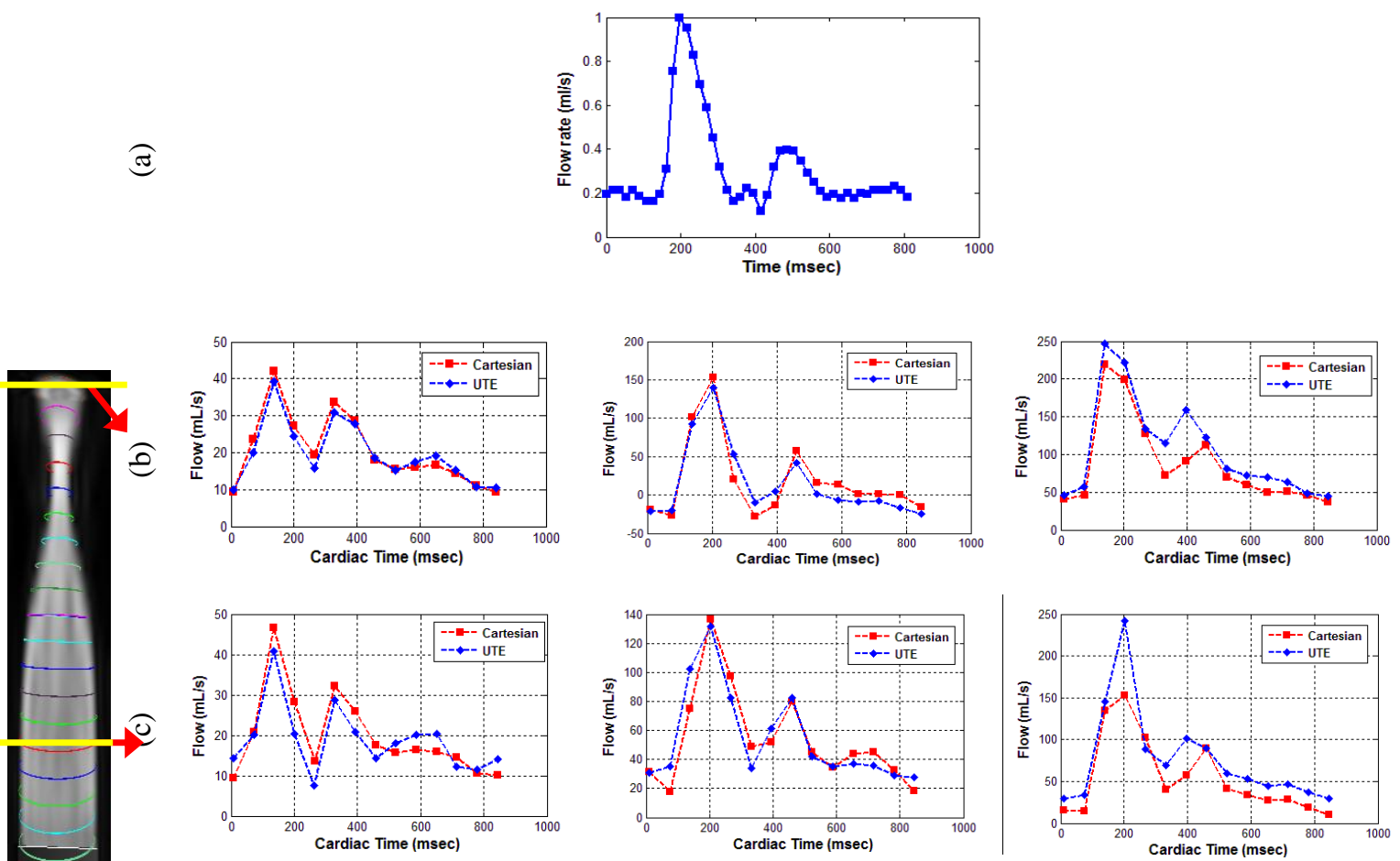

igure 55: Prescribed flow waveform (a). The peak systolic flow rate (as well as the entire waveform) is adjusted to reflect Qmax $=50 \mathrm{ml} / \mathrm{s}$ (first column), Qmax $=150 \mathrm{ml} / \mathrm{s}$ (second column), and Qmax $=300 \mathrm{ml} / \mathrm{s}$ (third column). Measured flow waveform in an axial slice $12 \mathrm{~mm}$ proximal to the stenosis (b) and $21 \mathrm{~mm}$ distal to the stenosis (c) In the Figure, conventional 4D flow MRI measurements have been plotted in red and 4D

UTE flow-derived measurements have been plotted in blue. The flow waveforms reveal reasonable correspondence at 50 and $150 \mathrm{ml} / \mathrm{s}$; however the conventional sequence result in an erroneous flow waveform and a significant underestimation of peak flow distal to narrowing due to turbulence and intravoxel dephasing.

The Shelley pump was used. 
shows the flow waveforms measured in a slice $12 \mathrm{~mm}$ proximal (b) and $21 \mathrm{~mm}$ distal (c) to the center of the narrowing at low, medium, and high flow rates. There is good correlation between 4D flow and 4D UTE flow-derived waveforms at low and medium flow rates, both at the proximal and the distal slice to the narrowing. However, at high flow rates, a clear underestimation of peak flow rate can be observed in the conventional 4D flow-derived waveform distal to the narrowing. The peak systolic flow rate from conventional 4D flow for the case of high flow rate in Figure 55 (c) displays significant underestimation. Note however that the measured peak systolic flow in all cases (Figure 55 (b-c) is lower than peak systolic flow at the pump (Figure 55 (a)). This is due to the compliance of the tubes which dampen the input flow waveform.

Figure 56 illustrates peak systolic flow rates along the length of the phantom for different

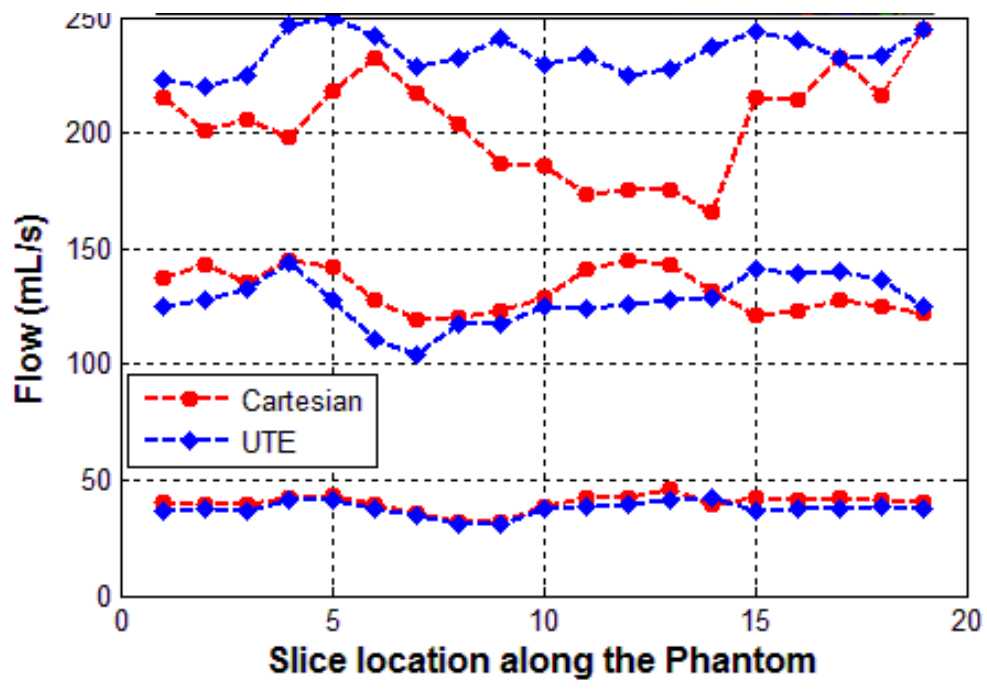

Figure 56: Measured peak systolic flow rate in slices along the length of the phantom for Qmax=50, 150, and $300 \mathrm{ml} / \mathrm{s}$ using conventional 4D flow (red) and 4D UTE flow (blue). There is good agreement between the two sequences with $\mathrm{Q}_{\max }=50$ and $\mathrm{Q} \max =150 \mathrm{ml} / \mathrm{s}$; however conventional 4D flow leads to a considerable variation in measured flow and a discernible underestimation distal to the narrowing. The Shelley pump was used. 
axial slices. Ideally, the plot for each peak flow rate should result in a straight line since the phantom is rigid. This is approximately the case for $50 \mathrm{ml} / \mathrm{s}$ and $150 \mathrm{ml} / \mathrm{s}$ pulsatile flow rates using both conventional 4D flow and 4D UTE flow techniques. However, for the case of high flow rate $\left(Q_{\max }=300 \mathrm{ml} / \mathrm{s}\right)$, the peak flow rates as derived from conventional $4 \mathrm{D}$ flow have a significant degradation and variation. As expected, the errors primarily occur at locations distal to the throat of the stenosis.

Figure 57 demonstrates the flow pathlines in a sagittal view of the phantom for the peak systolic flow of $\mathrm{Q}_{\max }=50 \mathrm{ml} / \mathrm{s}$ (top row) and $\mathrm{Q}_{\max }=150 \mathrm{ml} / \mathrm{s}$ (bottom row) pulsatile flow rates using conventional 4D flow MRI (a) and 4D UTE flow MRI(b). Flow pathlines for $\mathrm{Q}_{\max }=50$
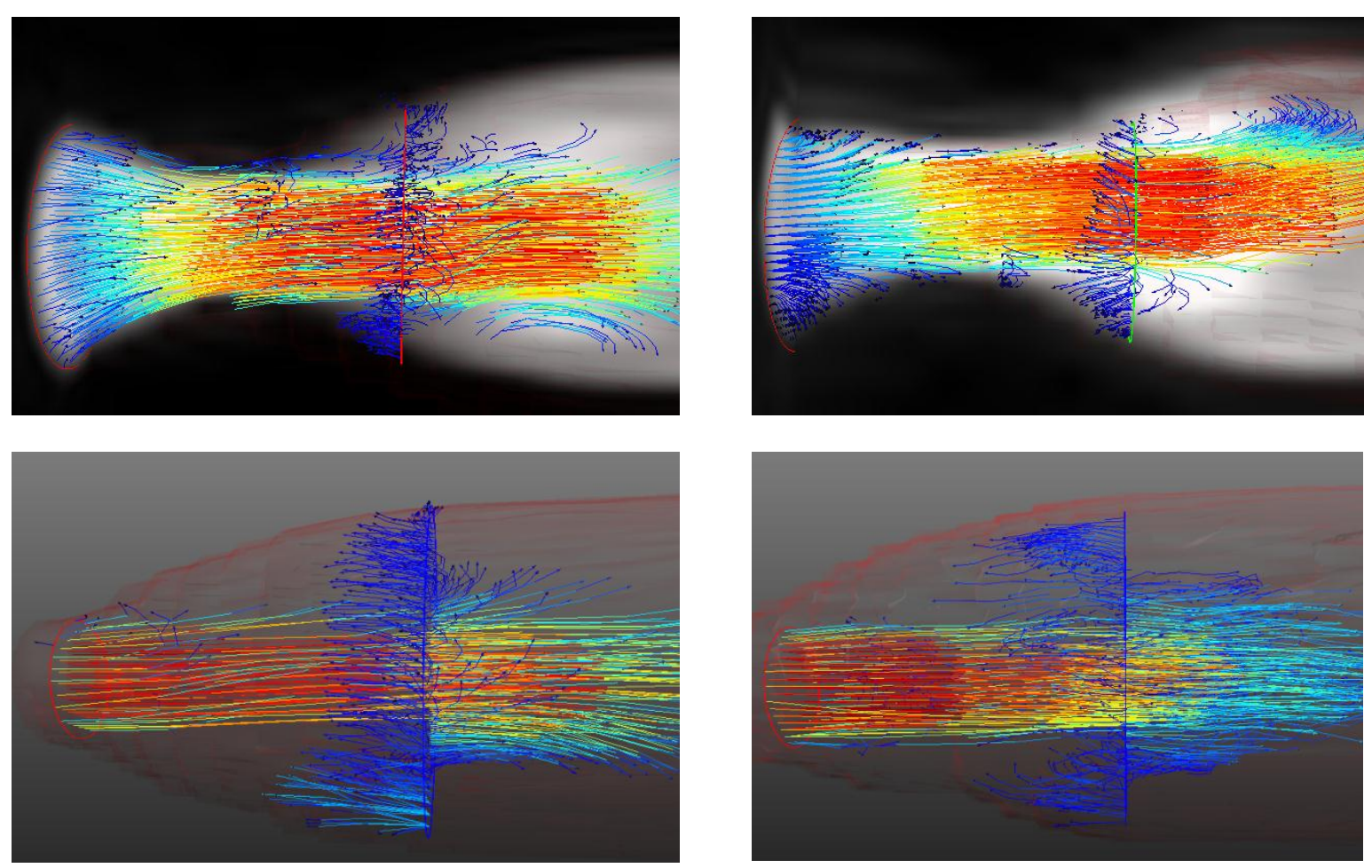

(a)

(b)

Figure 57: Flow pathlines in sagittal view at the pulsatile flow rates $50 \mathrm{ml} / \mathrm{s}$ (top row) and $150 \mathrm{ml} / \mathrm{s}$ at peak systolic time(bottom row) using conventional 4D flow MRI (a) and 4D UTE flow MRI (b). The flow pathlines are in good agreement at $50 \mathrm{ml} / \mathrm{s}$ flow rates. A slight difference is visible between flow pathlines at $150 \mathrm{ml} / \mathrm{s}$ due to moderately high flow rates and turbulence distal to the center of stenosis. The Shelley pump was used. 
$\mathrm{ml} / \mathrm{s}$ show good agreement between conventional 4D Flow MRI and 4D UTE flow MRI.

The correlation between the two results may be assessed based on the color-map as well as the flow pathlines. At $\mathrm{Q}_{\max }=150 \mathrm{ml} / \mathrm{s}$, a slight difference between flow pathlines obtained from the two sequences can be observed in locations distal to the stenosis where more significant spin dephasing occurs at this moderately high flow rate. Nevertheless, the correlation between the color-map and flow pathlines using the two sequences can be appreciated.

Figure 58 demonstrates the flow pathlines proximal to the stenosis, at the throat, and

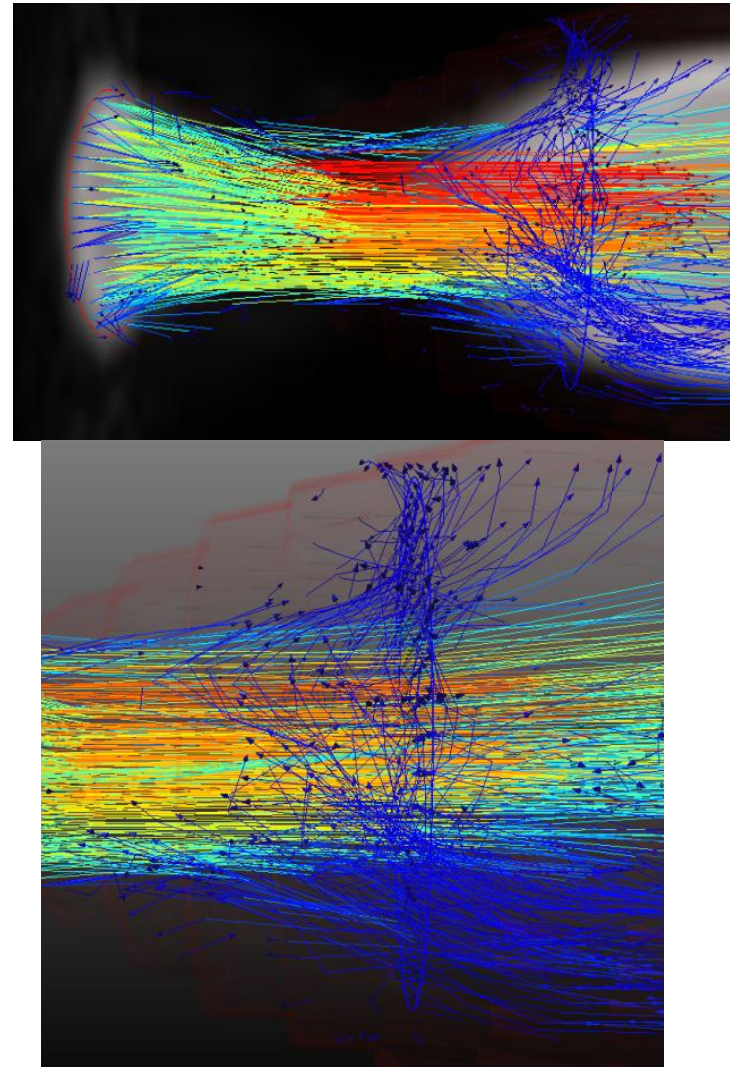

(a)

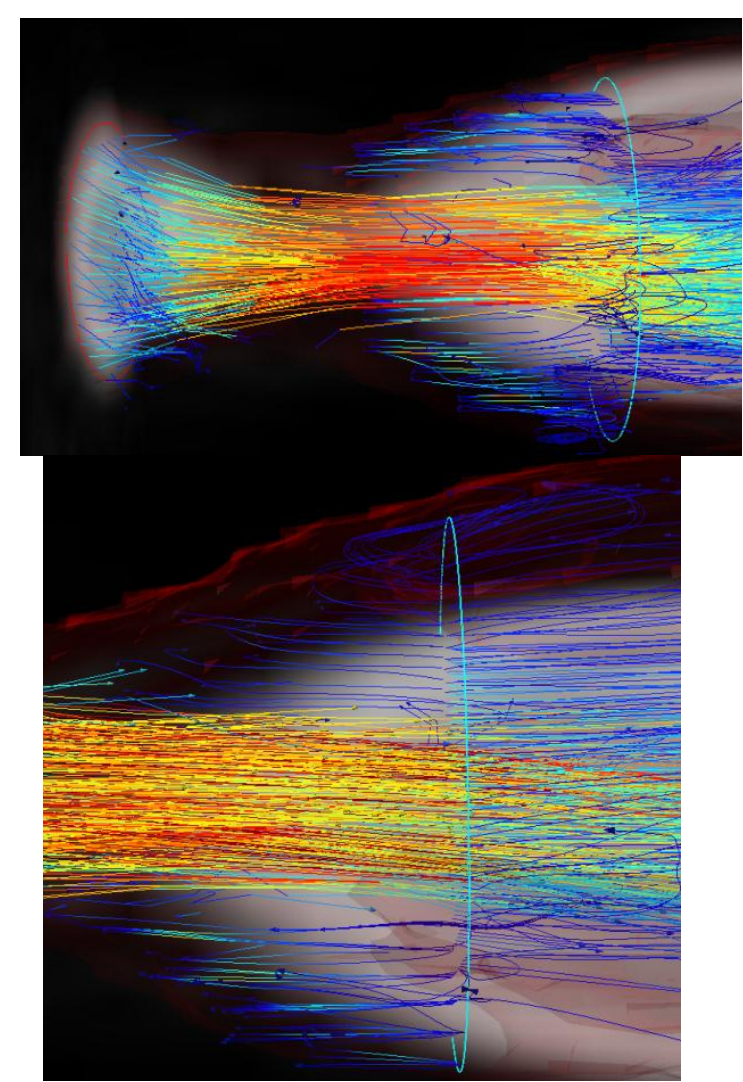

(b)

Figure 58: Flow pathlines at peak systolic time for the pulsatile flow with Qmax $=300 \mathrm{ml} / \mathrm{s}$ at peak systolic time using conventional 4D flow MRI (a) and 4D UTE flow MRI (b). The top row shows a sagittal view of the phantom including regions proximal and distal to the stenosis. The bottom row shows an enlarged view of distal regions. The error and inaccuracy in flow pathlines in the conventional sequence is evident. The Shelley pump was used. 
distal to the stenosis (top row) using conventional 4D flow MRI (a) and 4D UTE flow MRI (b) at for pulsatile flow with $\mathrm{Q}_{\max }=300 \mathrm{ml} / \mathrm{s}$ pulsatile flow rate and at peak systolic time. Due to intravoxel dephasing caused by high flow jet and turbulence, the flow pathlines derived with conventional 4D flow seem inaccurate. The bottom row shows an enlarged view of phantom distal to the throat of the stenosis.

\subsubsection{Validation Experiments with the LB pump}

In order to further validate the method and to test for dependence of results on different pump manufacturers, and additionally to test for the reproducibility of technique, some of the experiments performed with the Shelley pump were repeated with the LB pump with the same scanner field strength and coil.

Two sequences were used: conventional 4D flow MRI based on Cartesian trajectory and 4D UTE flow MRI based on the center-our radial trajectory. The common scan parameters for two sequences were $F O V=100 \times 100 \times 60 \mathrm{~mm}$, flip angle $=12$ degrees, and spatial resolution $=1.5 \times 1.5 \times 3.0 \mathrm{~mm}$ (leading to 20 axial slices). For pulsatile flow regimes, an ECG trigger signal was sent to the scanner with $\sim 1 \mathrm{~Hz}$ frequency and 14 frames were collected based on prospective ECG triggered scheme for the cardiac cycle. For the 4D UTE sequence $75 \%$ sampling of full k-space acquisition were selected. Also, Venc was constant for the entire length of the FOV along the phantom length (for the pulsatile flow rate with peak of $200 \mathrm{ml} / \mathrm{s}=300 / 75 / 75 \mathrm{~cm} / \mathrm{s}$, for pulsatile flow rate with peak of $300 \mathrm{ml} / \mathrm{s}=$ $450 / 100 / 100 \mathrm{~cm} / \mathrm{s}$ and for pulsatile flow rate with peak of $400 \mathrm{ml} / \mathrm{s}=600 / 125 / 125 \mathrm{~cm} / \mathrm{s}-$ note that the first number is Venc in the $\mathrm{z}$ direction and the next two numbers represent Venc for in-plane flow in the $\mathrm{x}$ and $\mathrm{y}$ directions). TE/TR for conventional 4D flow MR acquisitions were $4.79 / 10 \mathrm{~ms}$ (pulsatile flow rate $=200 \mathrm{ml} / \mathrm{s}$ ), 4.67/8 $\mathrm{ms}$ (pulsatile flow 
rate $=300 \mathrm{ml} / \mathrm{s}$ ), and $4.60 / 8 \mathrm{~ms}$ (pulsatile flow rate $=400 \mathrm{ml} / \mathrm{s}$ ) and for 4D UTE flow MRI TE/TR were $1.88 / 10 \mathrm{~ms}$ (pulsatile flow rate $=200 \mathrm{ml} / \mathrm{s}$ ), $1.69 / 8 \mathrm{~ms}$ (pulsatile flow rate $=300 \mathrm{ml} / \mathrm{s}$ ), and $1.55 / 8 \mathrm{~ms}$ (pulsatile flow rate $=400 \mathrm{ml} / \mathrm{s}$ )

To show the effect of phase error correction on UTE sequence when using the LB pump (similar to what was reported in Figure 49 when using the Shelley pump), Table 12 shows the calculated mean flow (over the entire cardiac cycle) at each slice along the phantom at

Table 12: Mean flow (over the entire cardiac cycle) at each slice along the phantom using conventional 4D flow MRI and 4D UTE flow MRI for the flow-on and flow-off experiments and the final result after phase error correction through subtraction of flow-off data from flow-on data. The unit for measurement is $\mathrm{ml} / \mathrm{s}$.

The LB pump was used.

\begin{tabular}{|c|c|c|c|c|}
\hline Slice & $\begin{array}{c}\text { Conventional 4D flow } \\
\text { MRI }\end{array}$ & $\begin{array}{c}\text { Flow-on 4D UTE } \\
\text { flow MRI }\end{array}$ & $\begin{array}{c}\text { Flow-off 4D UTE } \\
\text { flow MRI }\end{array}$ & $\begin{array}{c}\text { Final 4D UTE } \\
\text { derived }\end{array}$ \\
\hline 1 & 47.01 & 64.61 & 11.86 & 52.74 \\
\hline 2 & 45.35 & 66.32 & 12.72 & 53.59 \\
\hline 3 & 44.95 & 62.54 & 10.40 & 52.14 \\
\hline 4 & 45.92 & 58.33 & 9.59 & 48.74 \\
\hline 5 & 46.37 & 58.79 & 8.51 & 50.28 \\
\hline 6 & 44.45 & 55.47 & 4.21 & 51.26 \\
\hline 7 & 40.87 & 49.73 & 2.19 & 47.53 \\
\hline 8 & 37.47 & 40.33 & 1.09 & 39.24 \\
\hline 9 & 53.62 & 54.20 & 0.87 & 53.33 \\
\hline 10 & 63.85 & 62.11 & -0.17 & 62.29 \\
\hline 11 & 63.382 & 59.60 & -0.67 & 60.27 \\
\hline 12 & 58.987 & 51.81 & -2.78 & 54.60 \\
\hline 13 & 56.93 & 42.01 & -4.74 & 46.76 \\
\hline 14 & 52.08 & 37.77 & -6.13 & 43.90 \\
\hline 15 & 50.35 & 37.27 & -7.03 & 44.30 \\
\hline 16 & 45.89 & 35.77 & -13.2 & 49.04 \\
\hline 17 & 43.99 & 30.16 & -18.2 & 48.40 \\
\hline 18 & 38.95 & 24.09 & -24.8 & 48.95 \\
\hline 19 & 36.70 & 15.80 & -33.5 & 49.39 \\
\hline 20 & 39.15 & 12.63 & -37.7 & 50.42 \\
\hline
\end{tabular}


pulsatile flow rate of $\mathrm{Q}=200 \mathrm{ml} / \mathrm{s}$ using Conventional 4D flow MRI and 4D UTE flow MRI in both flow-off and flow-on pump experiments. The measured flow-on UTE at each slice reveals an error compared to measured flow using conventional technique. As before, by using a flow-off scan when running the 4D UTE sequence with identical imaging parameters, the error may be corrected as shown in the final UTE-derived results. The derived flow rates show good consistency with the expected mean flow at this flow rate.

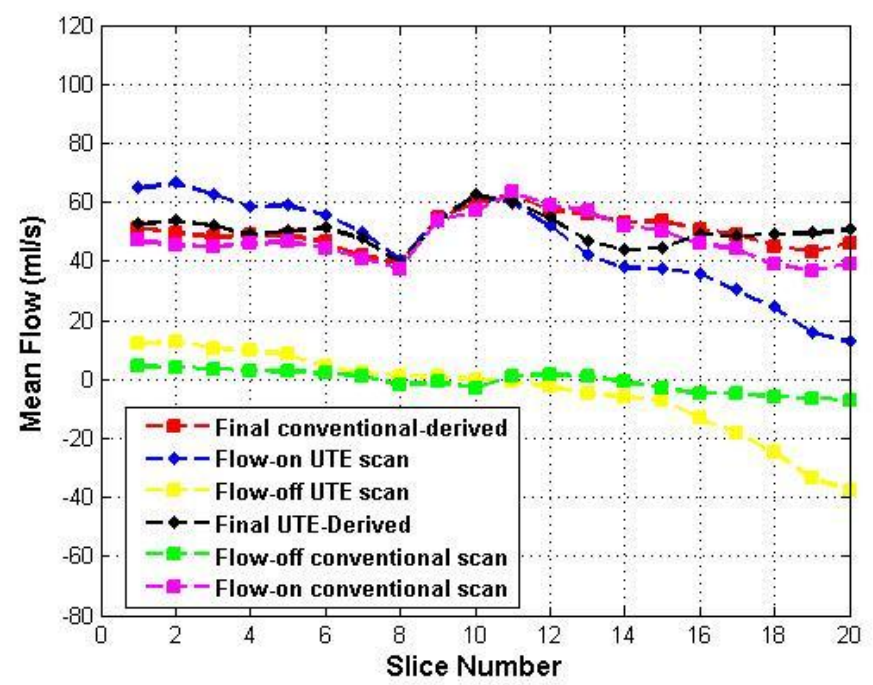

Figure 59: Mean flow (over the entire cardiac cycle) at each slice along the phantom at $\mathrm{Q}=200 \mathrm{ml} / \mathrm{s}$ using conventional 4D flow MRI and 4D UTE flow MRI with flow-on and flow-off experiments and the result after phase error correction. The LB pump was used.

Figure 59 demonstrates a sagittal reconstruction of the through-plane velocities for the case of $Q=200 \mathrm{ml} / \mathrm{s}$ pulsatile flow rate similar to Figure 49 for steady flow rate using Shelley pump. In Figure 59, the red and blue plots display flow rates as a function of position along the length of the phantom for each of the Cartesian and UTE scans. The yellow plot displays the flow-off scan which was used to reduce the flow error. This plot was subtracted from the UTE plot to correct the phase offset due to the field inhomogeneity. The black plot shows results from the UTE sequence after phase correction -- a good correlation between phase- 
corrected UTE scans and phase-corrected conventional scans are observed. The Figure follows the result acquired for $\mathrm{Q}=150 \mathrm{ml} / \mathrm{s}$ with Shelley pump (Figure 49) with a slight difference that the few first slices in the volume have more errors and offset from zero. That might be due to that fact that for the experiment using LB pump pulsatile flow rate were studied and mean flow was reported in Figure 60 (while Figure 49 was based on steady flow). More importantly, for LB pump experiments were performed on a different scanner which resulted in different field inhomogeneity and therefore phase errors.

Figure 61 demonstrates the mean velocity acquired at three locations proximal, at the stenosis, and distal to the center of the stenosis using conventional 4D flow and 4D UTE flow MRI with flow-off and flow-on scans. It is evident that after phase correction the UTE plots has a good correlation with conventional technique at all locations.

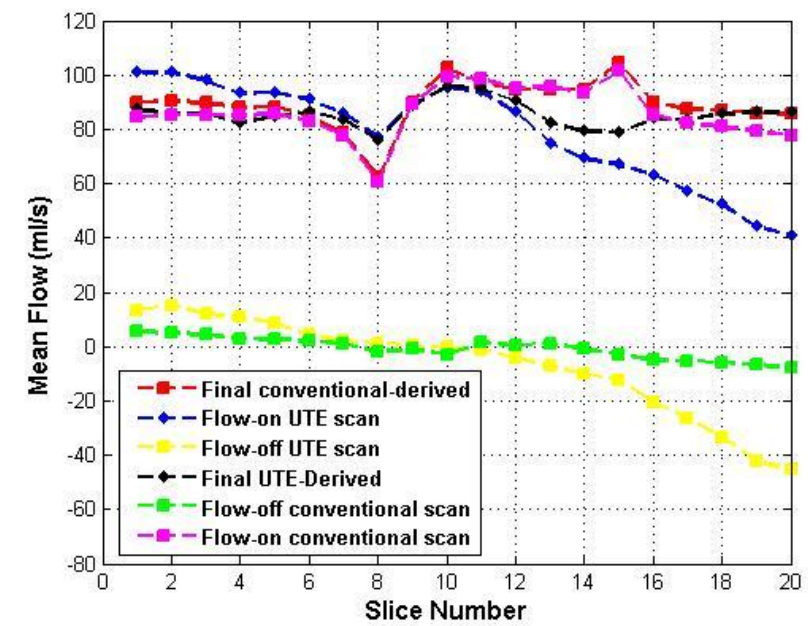

Figure 60: Mean flow (over the entire cardiac cycle) at each slice along the phantom at $\mathrm{Q}=300 \mathrm{ml} / \mathrm{s}$ using conventional 4D flow MRI and 4D UTE flow MRI with flow-on and flow-off experiments and the result after phase error correction. The LB pump was used. 


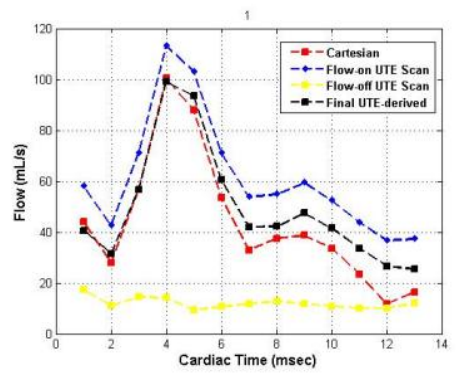

(a)

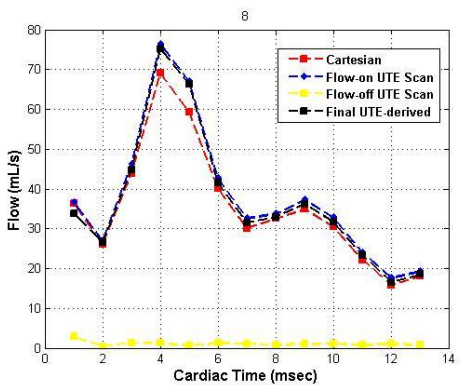

(b)

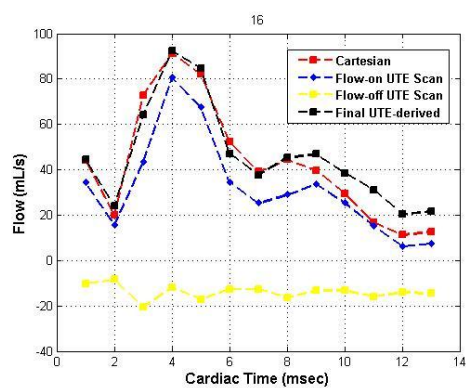

(c)

Figure 61: Flow waveform measured using conventional 4D flow MRI and 4D UTE flow MRI at three locations proximal (a), at the stenosis (b) and distal to stenosis (c). The LB pump was used.

Figure 60 and Figure Figure 62 show similar results using mean pulsatile flow at $Q=300$ $\mathrm{ml} / \mathrm{s}$ when using the LB pump.

Figure 63-Figure 65 show the velocity profile and velocity contour in axial cross sections of phantom proximal, at the throat, and distal to stenosis at peak systolic time (LB pump was used). The conventional 4D flow MRI (first row) and 4D UTE flow MRI (second row) were used to acquire velocity profile and contour from pulsatile flows at $Q=200,300$, and $400 \mathrm{ml} / \mathrm{s}$ peak flow rate. From the Figure higher velocity jet and fluctuation appears distal to stenosis, resulting in difference between Conventional and UTE techniques. In addition, the velocity pathlines distal to stenosis at the very right side of Figures (yellow arrows) point to the difference between jet length at different flow rate and increase in the length of velocity jets at higher flow rates. One may notice that the flow related artifact using this pump at high flow rates is less than the results using Shelley pump (please see Figure 58 for $Q=300 \mathrm{ml} / \mathrm{s}$

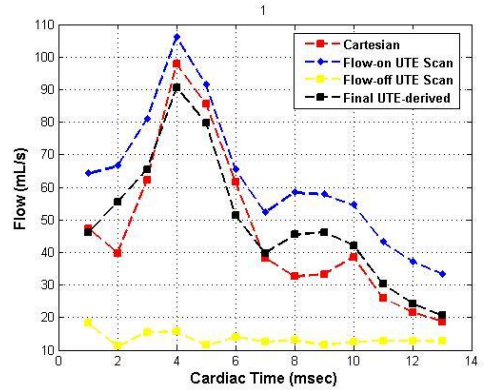

(a)

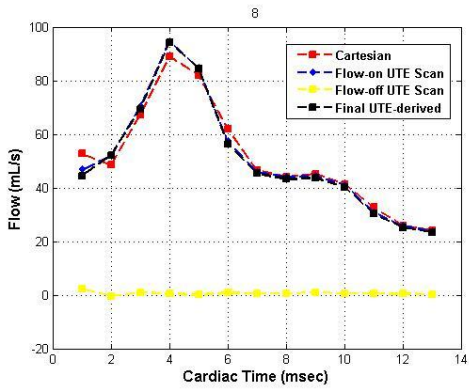

(b)

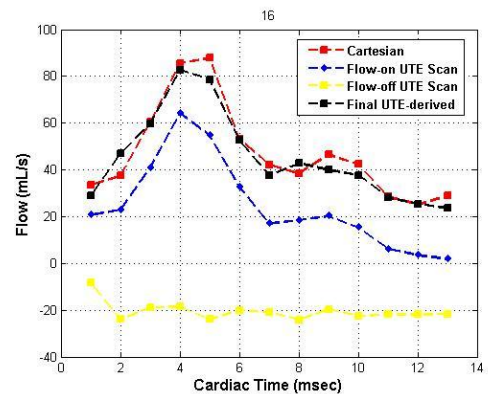

(c)

Figure 62: Flow waveform measured using conventional 4D flow MRI and 4D UTE flow MRI at three locations proximal (a), at the stenosis (b) and distal to stenosis (c). The LB pump was used. 
using Shelley pump). The main reason for less flow artifact is that, the actual peak velocity achieved in the phantom tube using LB pump had a significant damping ( $\sim 50 \%)$ compared to prescribed value at the pump.
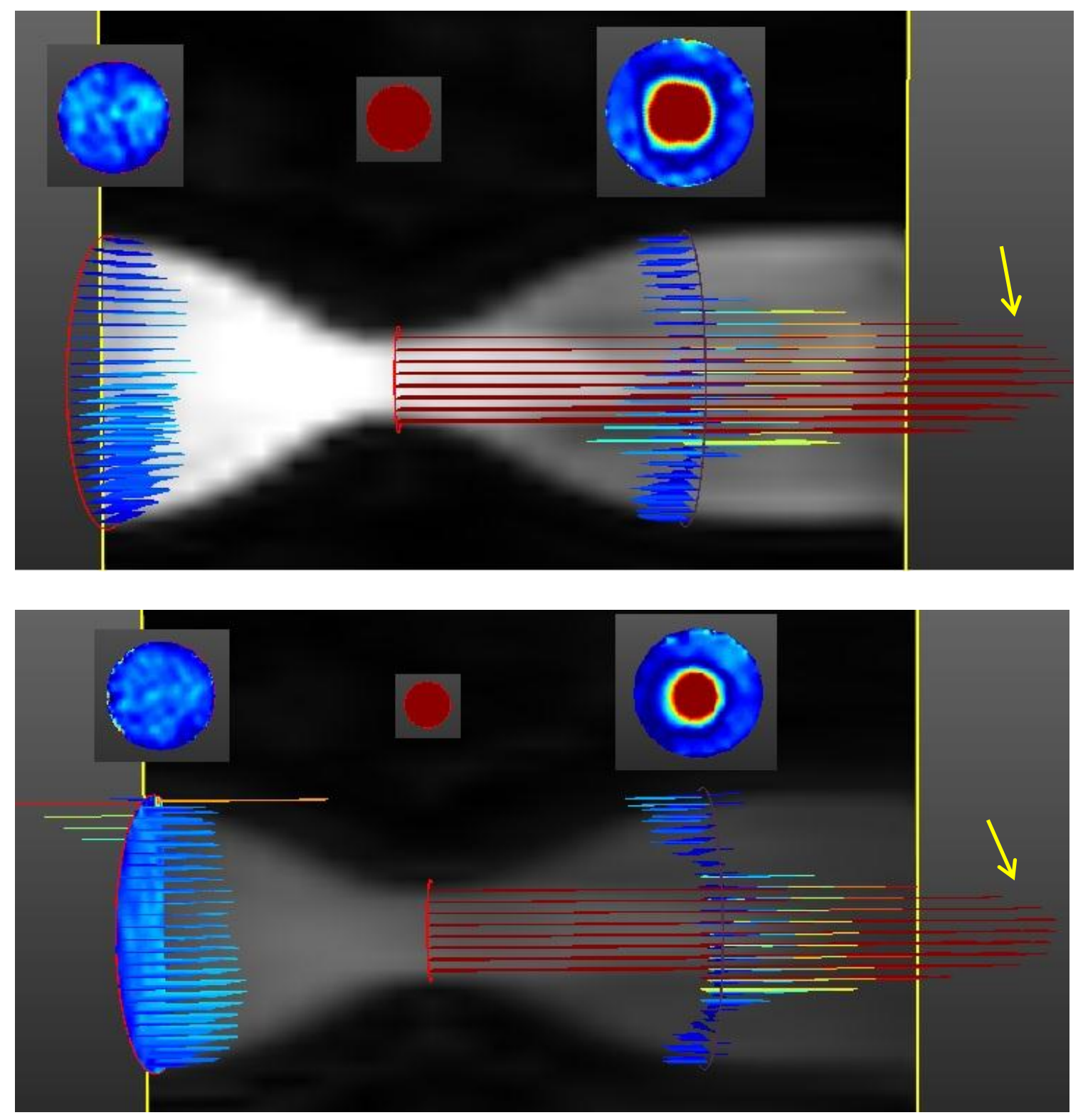

Figure 63: A sagittal view of phantom at $\mathrm{Q}=200 \mathrm{ml} / \mathrm{s}$ pulsatile flow rate at peak systolic time. The velocity profile and velocity contour at axial cross sections proximal, at the throat, and distal to narrowing are shown using conventional 4D flow MRI (first row) and 4D UTE flow MRI. Velocity profiles and contours reveal a good correlation between the two sequences. The LB pump was used. The darker phantom in the bottom figure is due to windowing level in the visualization software. 

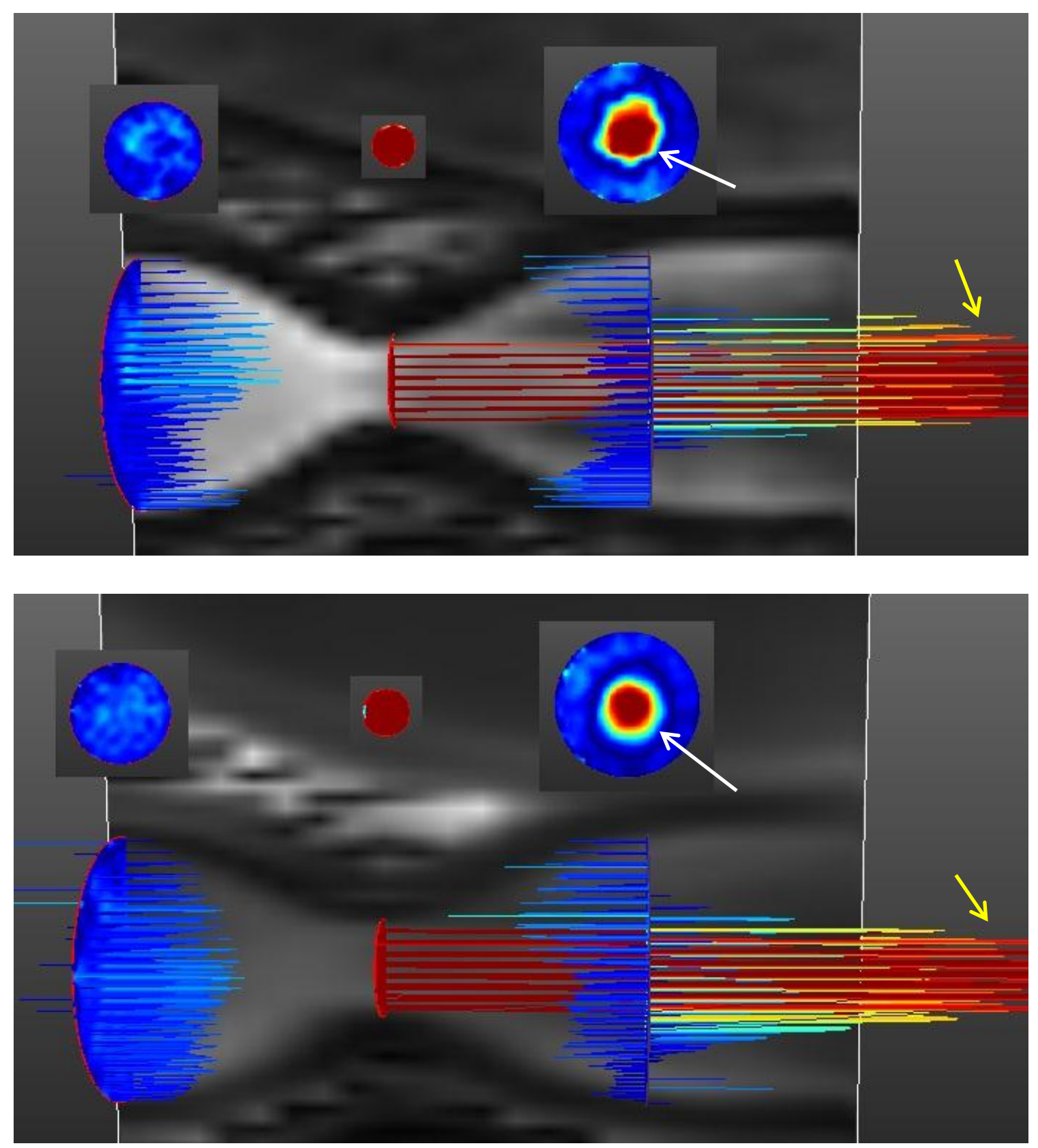

Figure 64: A sagittal view of phantom at $\mathrm{Q}=300 \mathrm{ml} / \mathrm{s}$ pulsatile flow rate at peak systolic time. The velocity profile and velocity contour at axial cross sections proximal, at the throat, and distal to narrowing are shown using conventional 4D flow MRI (first row) and 4D UTE flow MRI (second row). Velocity profiles and contours reveal a good correlation between two sequences. The difference between two sequence distal to stenosis is slightly more than $\mathrm{Q}=200 \mathrm{ml} / \mathrm{s}$ due to higher flow rate. The $\mathrm{LB}$ pump was used. 

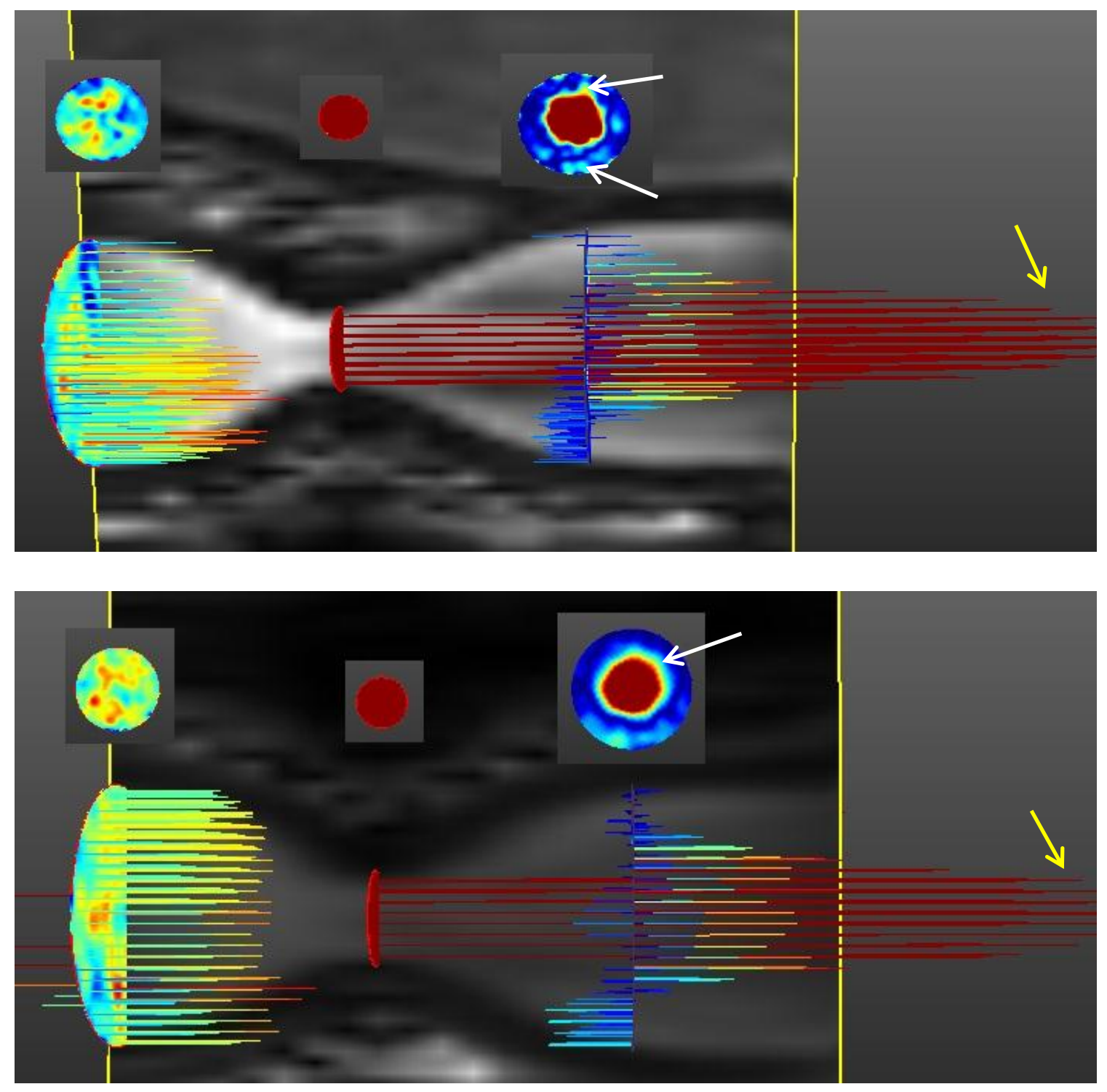

Figure 65: A sagittal view of phantom at $\mathrm{Q}=400 \mathrm{ml} / \mathrm{s}$ pulsatile flow rate at peak systolic time. The velocity profile and velocity contour at axial cross Sections proximal, at the throat, and distal to narrowing are shown using conventional 4D flow MRI (first row) and 4D UTE flow MRI (second row). Velocity profiles and contours reveal a good correlation between two sequences. The difference between two sequence distal to stenosis is more than $\mathrm{Q}=200$ and $300 \mathrm{ml} / \mathrm{s}$. The LB pump was used.

\subsection{Discussion}

A novel 4D UTE flow MRI technique was presented which benefits from a significantly shorter TE relative to conventional 4D flow MRI. The phantom measurement for the steady flow regime with various flow rates revealed good correlation between measured flow from 
conventional and UTE sequences at low and medium flow rates (Figure 52). At high flow rates, however, the conventional sequence resulted in intravoxel dephasing and signal loss causing flow related artifact in velocity contours (Figure 54). Similarly, validations and comparisons were performed for pulsatile flows and results concurred with those of steady flow tests for both the low and medium flow rates. Table 3 shows similar RRMSE's between conventional and UTE sequences for pulsatile flow rates of 14, 39, 50 and $150 \mathrm{ml} / \mathrm{s}$.

At high flow rates of 250 and $300 \mathrm{ml} / \mathrm{s}$ however, when compared to the conventional sequence, the UTE sequence performs more accurate flow quantification. The measured flow using conventional sequence at these flow rates showed a significant underestimation. The linear regression analysis (Figure 54) and the flow waveforms at high flow rates in Figure 55 confirm the flow quantification results and show the difficulty of the conventional approach in detecting the peak in the flow waveform. Both quantitative and flow waveform results for the UTE sequence reveal significant improvements when compared to the conventional sequence.

Streamline analysis and visualization of steady (Figure 53Figure 55) and pulsatile (Figure 57Figure 58) flow regimes at high flow rates also revealed significant errors distal to the narrowing in the presence of turbulence when using the conventional technique. Turbulence leads to signal loss in the magnitude image and intravoxel dephasing in the phase image.

Streamline analysis and visualization of steady (Figure 52) and pulsatile (Figure 56) flow regimes at high flow rates also revealed significant errors distal to the narrowing in the presence of turbulence when using the conventional technique. Turbulence leads to signal loss in the magnitude image and intravoxel dephasing in the phase image. 


\section{PATIENT STUDIES}

\subsection{Introduction}

Aortic stenosis (AS) is a hemodynamically age-related progressive disease which occurs at the opening of the aortic valve with symptoms related to the degree of valvular stenosis. In the majority of people with mild to moderate stenosis, no symptoms are present. Patients with severe aortic valve stenosis become symptomatic with syncope, chest pain, heart failure and they have a risk of sudden cardiac death possibly due to left ventricular hypertrophy that develops as a result of increase in the after-load. Although other causes of aortic stenosis exist, the most relevant cause is presence of age-related progressive calcification of the normal three-leaflet aortic valve. Echo Doppler and Catheterization are the mainstay for diagnosis. AS is classified as mild $\left(<25 \mathrm{~mm} \mathrm{Hg}\right.$ pressure drop, $>1.5 \mathrm{~cm}^{2}$ area), moderate (25-40 mm Hg pressure drop, 1.0-1.5 $\mathrm{cm}^{2}$ area), severe (> $40 \mathrm{~mm} \mathrm{Hg}$ pressure drop, $>0.75-$ $1.0 \mathrm{~cm}^{2}$ area), and critical ( $>70 \mathrm{~mm} \mathrm{Hg}$ pressure drop, $<0.75 \mathrm{~cm}^{2}$ area). Guidelines for assessment of AS severity for velocities depend on LV function with velocities $<2 \mathrm{~m} / \mathrm{s}$ with

normal LV function being appropriate, while in $2-4 \mathrm{~m} / \mathrm{s}$ requiring further quantification and analysis, especially with poor LV function and finally, velocities $>4 \mathrm{~m} / \mathrm{s}$ with normal LV function point to severe AS.

The clinical indications for cardiovascular MR for valvular heart disease was stated in a consensus panel report in 2004: 
"The low cost, flexibility and ease of handling make transthoracic echocardiography the primary clinical tool for evaluation of valvular heart disease. Moreover TEE is superior to CMR in assessment of valve morphology and detection of small and rapidly moving vegetation attached to the valves in endocarditis. However, CMR may play a complementary role when transthoracic acoustic windows are poor and a TEE approach is undesirable, or when results of echocardiography and catheterization are conflicting. Furthermore, CMR is a valuable tool for individual follow-up of the severity of regurgitant lesions and for quantification of the effects of valvular lesions on ventricular volumes, function, and myocardial mass ..."

Doppler Ultrasound is commonly used for cardiac valve assessment is Peak velocity and pressure gradient are calculated at the level of valve and used to determine the severity of stenosis [176]. However Doppler Ultrasound has several disadvantages including inaccurate measurement of valve area, LV outflow, flow velocity etc. due to low acoustic window and poor image quality [177]. PC MRI is another recognized method for flow quantification and assessment through the cardiac valves. This method can accurately quantify and characterize different valve related diseases such as Aortic Stenosis (AS) [7, 166, 178, 179] and Aortic Regurgitation (AR) [180-182].

Several groups have reported flow quantification and peak velocity measurement $[5,124$, 168], effective valve area for aortic valve $[6,54,166,178,179,183,184]$, and pressure gradients calculation [185] using PC MRI. These studies compared PC MRI with Doppler ultrasound and revealed a good agreement between these techniques but all of the used limited size of subjects with mild or moderate aortic stenosis. 
In [6] Sondergaards et al., studied 12 patients with aortic stenosis using Doppler ultrasound and conventional 2D PC MRI with TE $=3.5 \mathrm{msec}$ and in most of the subjects the measured peak velocities using PC MRI were underestimated compared to Doppler ultrasound. In a similar study, PC MRI with spiral trajectory were used to measure peak velocity in 4 patients with aortic stenosis wherein the peak velocity was up to $420 \mathrm{~cm} / \mathrm{s}$ and measurement showed accuracy of technique while an underestimation compared to Doppler echo was still inevitable [5].

Kinler et al investigated 29 patients with aortic and mitral valve stenosis using both conventional 2D PC MRI with TE=3.6 msec and Doppler echo and reported a good agreement between the two techniques [124]. However, in 7 patients the peak velocity measured using Doppler ultrasound was higher than $400 \mathrm{~cm} / \mathrm{s}$ and in 3 patients the measured peak velocity using two techniques were different.

In another study by Caruthers et al, 24 patients with aortic valve stenosis were investigated using conventional 2D PC MRI with $\mathrm{TE}=2.9 \mathrm{msec}$ and peak velocity at the level of the aortic valve was compared with Doppler ultrasound [166]. This study revealed an underestimation in measured peak velocity and aortic stenosis severity compared to Doppler ultrasound when the velocity time integral was higher than 0.8 meters. In more than half of the patients with severe aortic stenosis, peak velocities were underestimated when using PC MRI.

In another study, Waters et al. studied 23 patients with aortic stenosis using conventional 2D PC MRI with TE $=2.9$ msec and Doppler and showed a good agreement between measured flow and velocity profiles in the aortic root when using PC MRI and Doppler ultrasound [179]. However, in this study, only one patient had peak velocity higher than 400 
$\mathrm{cm} / \mathrm{s}$ and in this patient the difference between measured peak velocity using PC MRI and Doppler ultrasound was considerable.

Garcia et al. [186] investigated 31 patients with mild to severe aortic stenosis using conventional 4D PC MRI with TE=3.4 msec and Doppler ultrasound and reported that underestimation of LVOT cross sectional area by Doppler is compensated by overestimation of Velocity-time integral of LVOT, resulting in a good agreement between Doppler and PC MRI for estimation of aortic valve (EOA). In addition, MRI was associated with less intra and inter- observer measurement variability compared to Doppler ultrasound. The limitation of this study was that a few number of patients had severe stenosis.

Although several studies have reported use of PC MRI for evaluation of AS, the collected data with current technology is hampered due to the presence of turbulent jet distal to the narrowing and the resulting signal loss and image artifacts. This problem has been especially observed in the case of patients withsevere/critical aortic stenosis where the velocity measurement is less reliable $[166,168,179,184]$. Several approaches have been developed to potentially reduce the signal loss in PC MRI [4-7]. One important approach that appears to yield significant improvements and to correct the signal loss involves reduction of the echo time (TE) and gradient duration [8-11].

Nayak et al. [5] investigated 4 patients with mild-moderate aortic stenosis using a 2D Spiral PC MRI technique with TE $=2 \mathrm{msec}$ and Doppler echo. A good agreement between measured peak velocity using spiral PC MRI and Doppler echo was reported. They claimed that this technique is capable of imaging through-plane velocities up to $10 \mathrm{~m} / \mathrm{s}$ but the the peak velocity in patients did not exceed $420 \mathrm{~cm} / \mathrm{s}$. 
O'Brien et al presented a 2D thorugh-plane UTE PC MRI to reduce the intravoxel dephasing by shortening TE to $0.65 \mathrm{msec}$ [12]. A stenotic phantom as well as a normal volunteer and 2 patients with moderate to severe aortic stenosis were investigated. The mean velocity in the aortic valve was compared between UTE PC MRI and conventional PC MRI and revealed a good agreement however the results were not validated using Doppler ultrasound. Other problems with this technique were explained earlier in Section 5.3.

Previously, it was shown that 4D UTE flow MRI technique with TE shorter than conventional PC MRI techniques can reduce the phase errors distal to stenosis in a flow phantom with high flow rates [13]. In this Chapter, a correlative study of flow measurement from 4D UTE flow compared to conventional 4D flow and Doppler Ultrasound for assessing velocity and flow in patients with AS is reported.

\subsection{Method}

\subsubsection{Patient population and cases}

The study was approved by the Louisville VA Institutional Review Board and involved validation of 4D UTE flow MRI sequences on the 1.5T scanner at Robley Rex VA medical center pursuant to a research agreement with Philips healthcare. Twelve patients with mild to moderate Aortic Stenosis (AS) and Aortic regurgitation (AR) were selected for Doppler ultrasound and MRI scans. Inclusion criteria were: 1) Evidence of Aortic valvular disease obtained by a noninvasive study including CTA, MRA and/or Doppler with a 50-90\% diameter stenosis, or regurgitation. 2) Study subjects to be in stable condition at the time of MRI study, and they should be able to lay flat for the duration of the exam (for about 1 hour). Exclusion criteria were: 1) age $<20$ or $>90$ years old, 2) unconscious or mentally 
unstable patients, 3) individuals such as pregnant women, prisoners, institutionalized individuals or those unable to give informed consent, 4) patients with severe hypertension (> $200 \mathrm{mmHg}$ systolic and/or $110 \mathrm{mmHg}$ diastolic), 5) weight more than $350 \mathrm{lb}, 6)$ chronic atrial fibrillation and arrhythmias precluding ECG gating, 7) claustrophobia, 8) any metallic implant including but not limited to cardiac pacemakers, defibrillator, cochlear implant, tissue expander, any aneurism clip, insulin pump, drug infusion pump, older mechanical heart valves (pre-6000 series Starr-Edward caged ball), metallic foreign bodies (such as gunshot, shrapnel, BB, ...), older orthopedic plates and screws, transdermal drug patches, penile implant or pump and prior metal fragments in the eye related to prior metal welding, or other contraindication of the MRI examination. All patients gave informed consent prior to enrolling in the study. Three patients were excluded from further investigation including one patient with very noisy data, one patient with obesity, and one with Claustrophobia. Another nine subjects underwent MRI scan and in eight, Doppler Echocardiography was performed as well.

\subsubsection{Imaging strategy}

Based on standard clinical protocol, and as part of initial evaluations, patients underwent Doppler echo study of Aortic valve. Doppler echo study and data analysis were performed by one experienced echocardiographer. MRI examinations were performed on a $1.5 \mathrm{~T}$ Achieva Philips scanner using a dedicated 5 channel SENSE cardiac coil. For the MRI study, the patient was positioned supine on the MRI Table and 6 lead ECG electrodes were attached to the skin in order to perform gated imaging. Additionally, in order to mitigate the effect of breathing artifacts on imaging, navigator gating was performed. Two separate scan techniques were used: conventional 4D flow MRI and the proposed 4D UTE flow MRI. Several scans were performed to determine the orientation of heart and aortic valve. The 
image volume was adjusted so that the aortic valve was located proximal to the center of the volume and slices were perpendicular to Left Ventricle Outflow Tract (LVOT). Ten contiguous slices starting from locations proximal to the valve, through the center of the valve, and distal to the valve were collected within a 3D volume. The scan parameters for two sequences were TE/TR $=2.9 / 6.9 \mathrm{~ms}$ (for conventional 4D MRI), TE/TR $=1.15 / 4.6$ $\mathrm{ms}$ (for 4D UTE MRI), Venc $=400$ in all three flow directions, flip angle $=10$, spatial resolution $=2.5 * 2.5 * 5.0 \mathrm{~mm}$. Depending on the size of subjects, FOV in the in-plane direction was varied but $50 \mathrm{~mm}$ in the through plane direction was kept constant for all subjects. For UTE sequence, 75\% sampling of radial k-space lines was performed to reduce the scan time. 16 cine cardiac phases were collected in each cardiac cycle with ECG triggering. The scan time for each 4D scan was about 4 minutes with a small variation between different patients based on their size and selected FOV. However, due to application of navigator gating and depending upon the patients' breathing consistency and performance, each scans took on the order of 10-15 minutes depending on navigator efficiency and breathing pattern for a particular subject. The entire MRI examination was limited to one hour.

\subsubsection{Data analysis}

MRI analysis was carried out at the aortic valve and the flow and peak velocity were measured at all cardiac frames and were compared with Doppler echo in 9 subjects. Other measured parameters are shown in Table 13.

Similar to flow phantom studies, described in Section 9.2.5, in order to perform phase correction, for all 4D flow acquisitions (conventional and UTE), a static phantom was scanned with the 4D conventional and 4D UTE sequences and the phase of each scan was 
subtracted from the corresponding phase of the 4D conventional and 4D UTE scans. In addition, for all 4D UTE acquisitions including in-vivo and static phantom scans, phase errors due to gradient delay and hardware imperfections were also corrected based on an auto correlation technique which was employed to measure the k-space trajectory delays [163] and was previously explained in Section 5.5 of the dissertation. The measured trajectory delays were used to correct the trajectory delay offset in all subsequent scans with identical parameters.

To derive the flow waveforms from MRI velocity data, the Region of Interest (ROI) in each frame at each slice was manually selected. Correlation and agreement between MRI techniques and Doppler echo were assessed using Bland-Altman analysis which is explained as the mean difference with the confidence range or limit agreement. The confidence range is the standard deviation of the difference between two techniques. The mean difference in Bland-Altman analysis may be describes the bias in the data. The spread of data displays systematic errors related to size of the measurement. In most cases, the LVOT diameters acquired in two directions using MRI are very close to each other but in general to compare to echo Doppler the average of diameters was calculated. All the post-processing and the flow assessments were performed using Matlab software (The Mathworks, Natick, MA).

\subsection{Results and discussions}

Table 13 shows the parameters calculated using conventional 4D flow, 4D UTE flow MRI and Doppler echo in all patients at the level of Aortic valve (Doppler echo was not collected in patient 5, 11, and 12). Peak velocity measured using conventional 4D flow MRI and 4D

UTE flow MRI compared to Doppler echo reveals a good agreement in all patients. Root 
Mean Squared Error (RMSE) was calculated by comparing the peak velocity of six patients measured using 4D UTE flow MRI and conventional 4D flow MRI with Doppler echo and the results are 6.9 for 4D UTE flow MRI and 10 for conventional 4D MRI. Figure 66 demonstrates the Bland-Altman plot representing the mean difference of peak velocity measured using conventional 4D flow (a) and 4D UTE flow MRI (b) in comparison with flow measured by Doppler echo. The Bland-Altman plot reveals a reasonable accuracy for 4D UTE flow with mean difference and confidence range at aortic valve as 8.1 and [-55.69, 39.46] and for conveniotnal 4D flow MRI was 5.1 and [-59.67, 65.87].

Figure 67Figure 69 display flow versus cardiac time for all subjects at the level of aortic valve (a) and $15 \mathrm{~mm}$ distal to the aortic valve (b) using conventional 4D flow MRI (red plot) and 4D UTE flow MRI (blue plot). In most of the subjects there is a good correlation between the two techniques. In subject 4, measured flow has an overestimation using conventional 4D flow MRI due to significant breathing artifact. 4D UTE flow MRI is less sensitive to breathing and motion artifact due to inherent nature of the radial acquisition. In subjects 5, 6, 7, 9 and 10, at both the level of the aortic valve and distal to the aortic valve, the flow waveforms for conventional 4D flow and 4D UTE flow MRI have good correlation. In all of these subjects, negative flows can be seen as the subjects have Aortic Regurgitation (AR) and the AR eject time for both conventional 4D flow and 4D UTE flow are very close to each other. In subject 9, UTE waveform distal to the stenosis shows variation in flow at different cardiac time due to more streaking artifact present in this subject's UTE data. One reason for this this may be that the location of the aortic artery in axially planned slices distal to the valve is more off-center in the volume due to inaccurate planning and it is well-known that the off-center regions of the image in radial acquisitions is 
more sensitive to streaking artifacts. In subject 8 , the flow waveform for conventional 4D flow

Table 13: Measured parameters using conventional 4D flow MRI, 4D UTE flow MRI, and Doppler echo technique in nine patients.

\begin{tabular}{|c|c|c|c|c|c|c|c|}
\hline $\begin{array}{c}\text { Patient } \\
\text { (disease) }\end{array}$ & Modality & Slice & $\begin{array}{c}\text { Average } \\
\text { Flow } \\
(\mathrm{ml} / \mathrm{s})\end{array}$ & $\begin{array}{c}\text { Peak } \\
\text { Velocity } \\
(\mathrm{cm} / \mathrm{s})\end{array}$ & $\begin{array}{c}\text { Time } \\
\text { Peak AV } \\
\text { (ms) }\end{array}$ & $\begin{array}{c}\text { AV Eject } \\
\text { Time } \\
\text { (ms) }\end{array}$ & $\begin{array}{c}\text { LVOT } \\
\text { Diameter } \\
(\mathrm{cm})\end{array}$ \\
\hline \multirow{3}{*}{$\begin{array}{l}\mathrm{P} 4 \\
(\mathrm{AS})\end{array}$} & Cartesian & 5 & 281.864 & 284.3 & 82 & 240 & 3.1 \\
\hline & UTE & 5 & 252.847 & 291.7 & 82 & 240 & 3.3 \\
\hline & Doppler & $\mathrm{AV}$ & -- & 277 & 60 & 260 & 2.4 \\
\hline \multirow{3}{*}{$\begin{array}{c}\mathrm{P} 5 \\
(\mathrm{AR})\end{array}$} & Cartesian & 5 & 113.129 & 140.4 & 95 & 360 & 2.6 \\
\hline & UTE & 5 & 163.439 & 210.7 & 95 & 350 & 2.8 \\
\hline & Doppler & $\mathrm{AV}$ & -- & -- & -- & -- & -- \\
\hline \multirow{3}{*}{$\begin{array}{c}\text { P6 } \\
\text { (AS/AR) }\end{array}$} & Cartesian & 5 & 137.81 & 310.0 & 102 & 350 & 3.2 \\
\hline & UTE & 5 & 254.224 & 325.3 & 102 & 360 & 3.1 \\
\hline & Doppler & $\mathrm{AV}$ & -- & 359 & 135 & 370 & 2.3 \\
\hline \multirow{3}{*}{$\begin{array}{c}\text { P7 } \\
(\mathrm{AS} / \mathrm{AR})\end{array}$} & Cartesian & 5 & 120.018 & 230.7 & 150 & 410 & 3.1 \\
\hline & UTE & 5 & 133.9133 & 250.3 & 150 & 380 & 3.1 \\
\hline & Doppler & $\mathrm{AV}$ & -- & 265 & 135 & 340 & 2.3 \\
\hline \multirow{3}{*}{$\begin{array}{l}\text { P8 } \\
\text { (AS) }\end{array}$} & Cartesian & 6 & 125.339 & 277.9 & 110 & 320 & 3.9 \\
\hline & UTE & 6 & 164.529 & 268.3 & 100 & 320 & 3.9 \\
\hline & Doppler & $\mathrm{AV}$ & -- & 255 & 77 & 310 & 2.3 \\
\hline \multirow{3}{*}{$\begin{array}{c}\text { P9 } \\
\text { (AS/AR) }\end{array}$} & Cartesian & 6 & 126.355 & 260.3 & 110 & 380 & 3.6 \\
\hline & UTE & 6 & 209.072 & 272.4 & 110 & 370 & 3.5 \\
\hline & Doppler & $\mathrm{AV}$ & -- & 246 & 100 & 380 & 2.3 \\
\hline \multirow{3}{*}{$\begin{array}{c}\text { P10 } \\
\text { (AS/AR) }\end{array}$} & Cartesian & 5 & 177.921 & 290.3 & 110 & 380 & 3.5 \\
\hline & UTE & 5 & 130.89 & 321.9 & 110 & 380 & 3.4 \\
\hline & Doppler & AV & -- & 328 & 100 & 340 & 2.3 \\
\hline \multirow{3}{*}{$\begin{array}{l}\text { P11 } \\
\text { (AS) }\end{array}$} & Cartesian & 3 & 168.66 & 328 & 100 & 300 & 2.7 \\
\hline & UTE & 3 & 206.43 & 332 & 100 & 300 & 2.8 \\
\hline & Doppler & $\mathrm{AV}$ & -- & 294 & 80 & 250 & -- \\
\hline \multirow{3}{*}{$\begin{array}{l}\text { P12 } \\
\text { (AS) }\end{array}$} & Cartesian & 4 & 185.95 & 353 & 180 & 380 & 2.8 \\
\hline & UTE & 4 & 187.52 & 362 & 150 & 380 & 2.8 \\
\hline & Doppler & $\mathrm{AV}$ & -- & 335 & 12.5 & 370 & -- \\
\hline
\end{tabular}




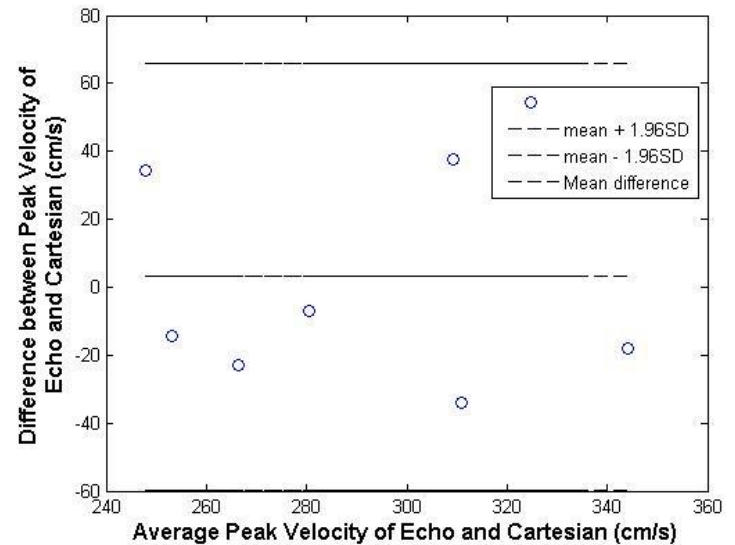

(a)

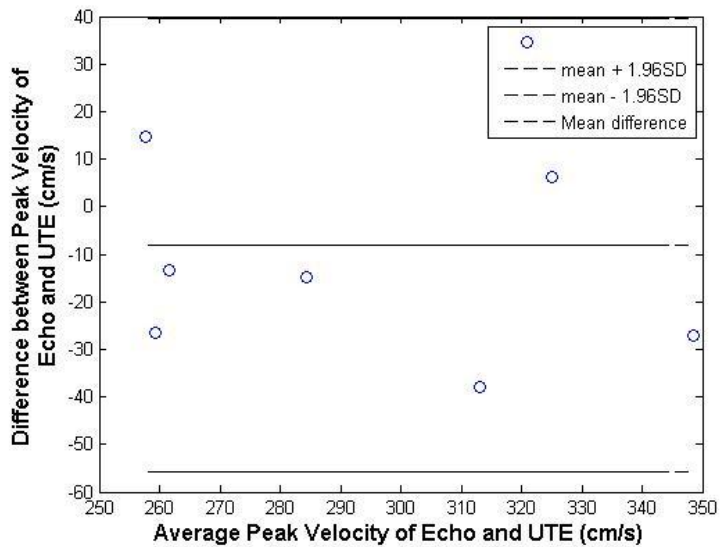

(b)

Figure 66: Bland-Altman plot at aortic valve in eightpatients demonstrating the peak velocity difference between 4D conventional (a) and 4D UTE flow (b) with Doppler echo.

MRI seems to be slightly less accurate since this subject had porcine valve replacement resulting in susceptibility artifact. This inaccuracy can be seen as incorrect negative flows (shown with black arrow in the second row of Figure 68(a)) as the subject did not have AR. 4D UTE flow MRI is less sensitive to susceptibility artifacts due to shorter TE and the flow waveforms look more reasonable. In Subject 11 and 12 with moderate AS, the waveforms using both techiques are similar. However, in subject 11, UTE waveform (blue plot) shows better consistency between the left and the right plots which represent the slice at the valve and the slice distal to valve while conventional waveform shows inaccuracies distal to valve where higher velocities are present.

LVOT diameters measured using conventional 4D flow and 4D UTE flow MRI were very similar for all patients (bias $=-0.016 \mathrm{~cm}$, agreement limits: -0.2458 to $+0.2125 \mathrm{~cm}$ ). However, the measurements using Doppler echo is smaller than conventional 4D flow (bias $=-1.0833 \mathrm{~cm}$, agreement limits: -1.7557 to $-0.4110 \mathrm{~cm}$ ) and 4D UTE flow MRI (bias = $1.0667 \mathrm{~cm}$, agreement limits: -0.4636 to $-1.6697 \mathrm{~cm}$ ) mainly due to assumption of circular LVOT in Doppler echo and using smaller diameter in AP direction to measure the area. 
However, with MRI no assumptions about the shape are required and one can use the actual shape of LVOT, resulting in more accurate measurements. In most of the cases, the LVOT
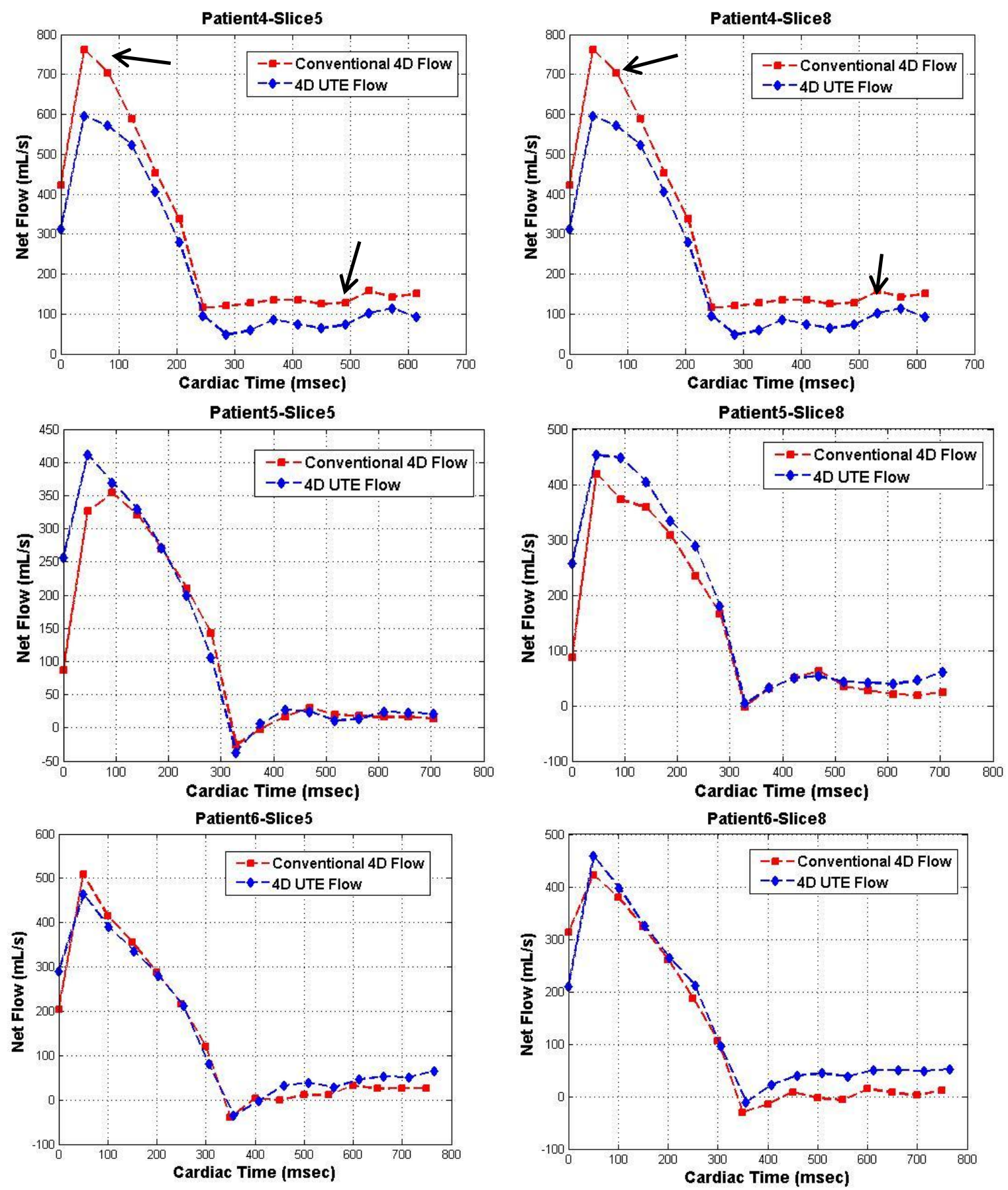

(a)

(b)

Figure 67: Flow versus time waveforms at the level of the aortic valve (a), and $15 \mathrm{~mm}$ distal to the aortic valve (b) in subject 4 with AS (first row), subject 5 with AR (second row), and subject 6 with AS and AR (third row) using conventional 4D flow MRI (red plot) and 4D UTE flow MRI(blue plot). 

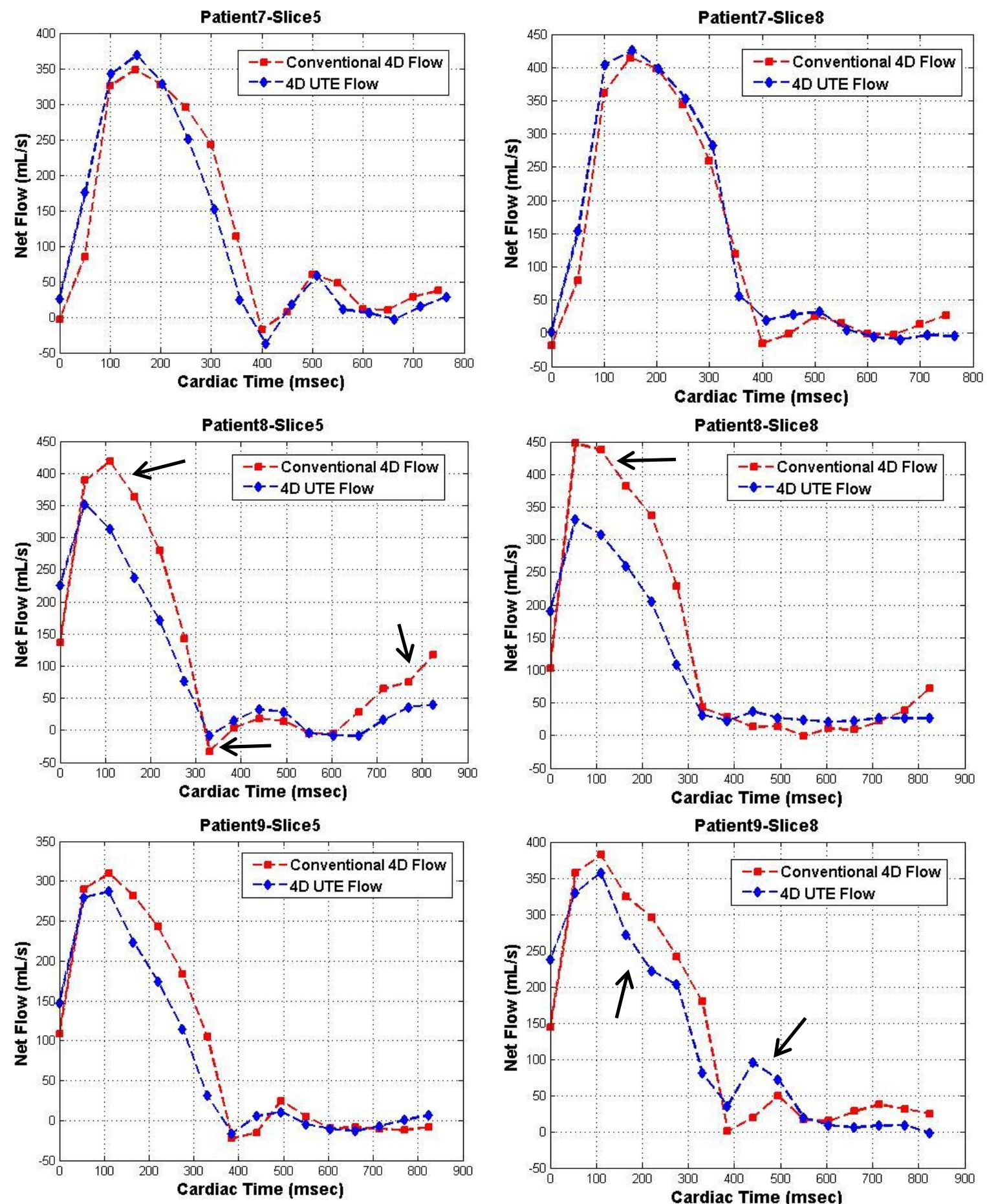

(a)

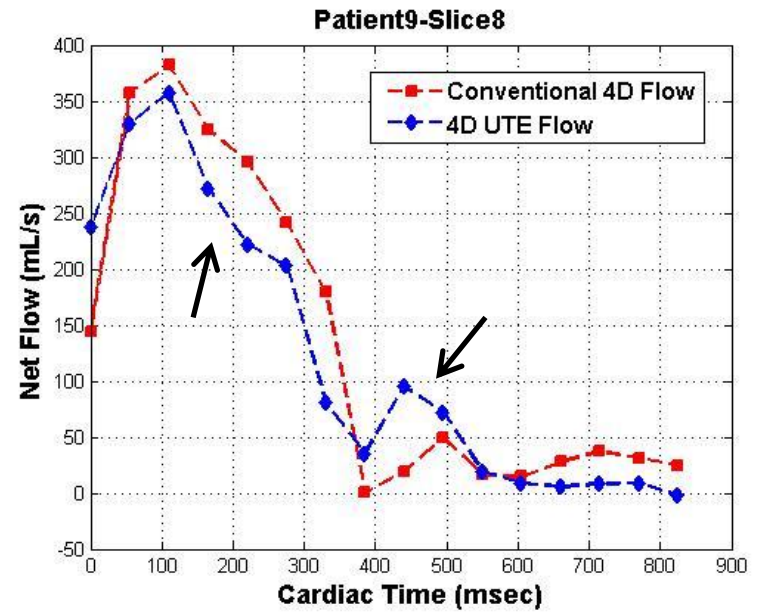

(b)

Figure 68: Flow versus time waveform at the level of the aortic valve (a), and $15 \mathrm{~mm}$ distal to the aortic valve (b) in subject 7 with AS and AR (first row), subject 8 with AS ( second row), and subject 9 with AS and AR (third row) using conventional 4D flow MRI (red plot) and 4D UTE flow MRI(blue plot). 
diameters in two directions calculated using both MRI techniques are very close. To compare measured diameters using MRI techniques to Doppler, the average of diameters in two directions was used. Figure 70 demonstrates the comparison between three techniques using Bland-Altman analysis.
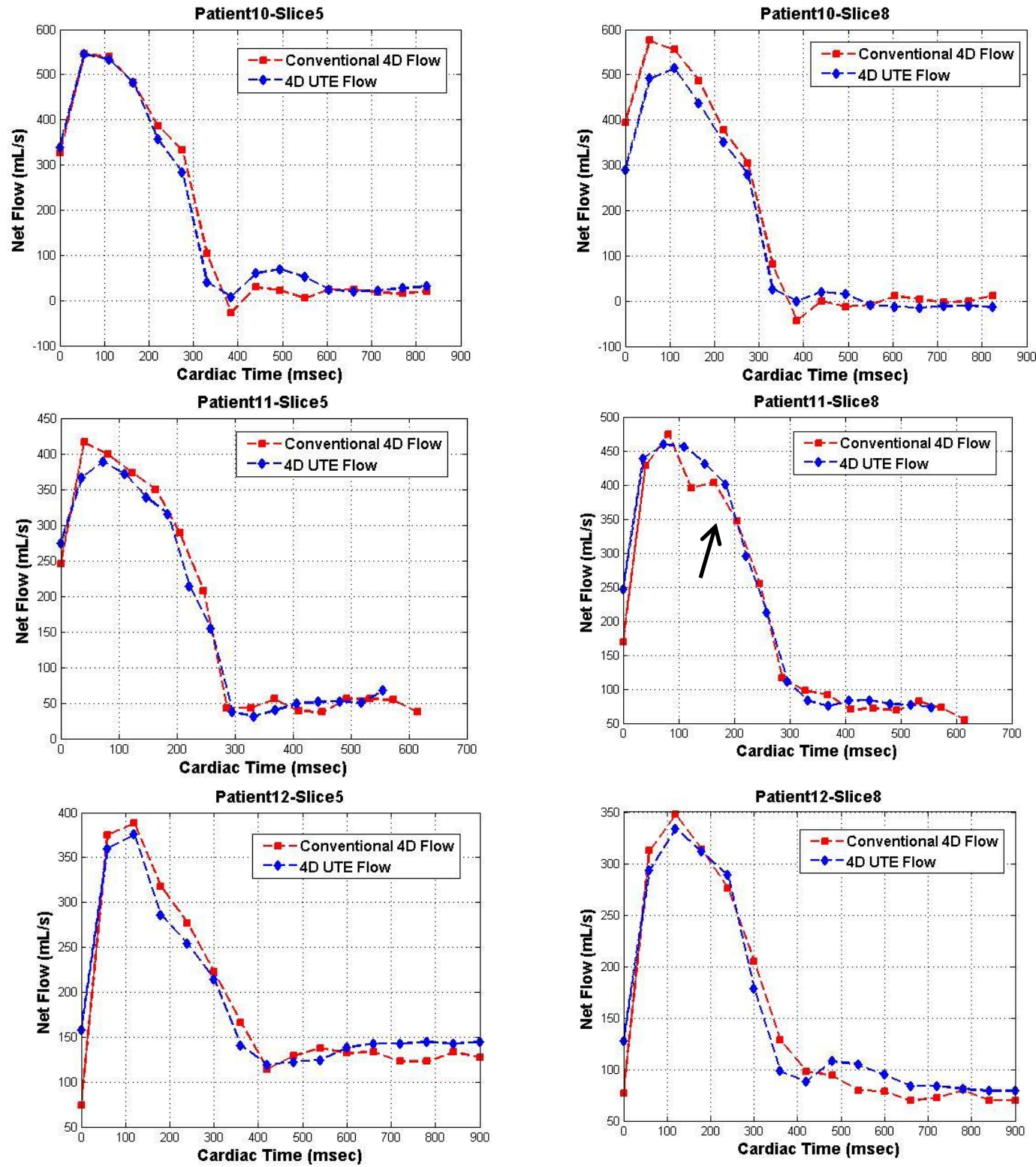

(a)

(b)

Figure 69: Flow versus time waveforms at the level of the aortic valve (a), and $15 \mathrm{~mm}$ distal to the aortic valve (b) in subject 10 with AS and AR (first row), subject 11 with AS (second row), and subject 12 with AS and AR (third row) using conventional 4D flow MRI (red plot) and 4D UTE flow MRI (blue plot). 


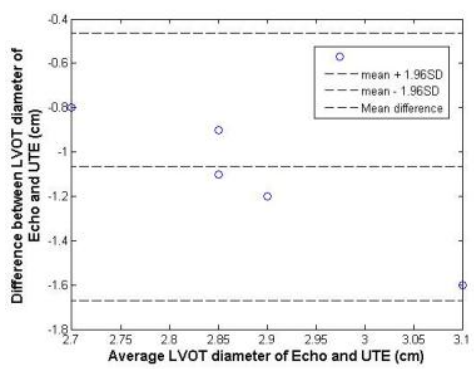

(a)

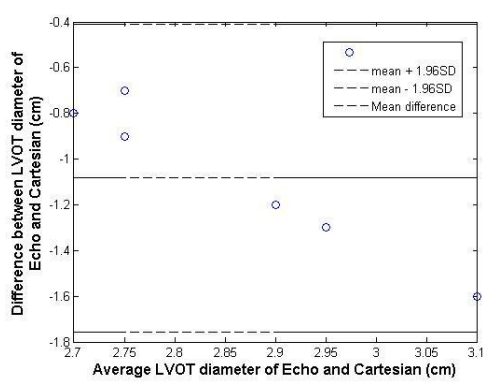

(b)

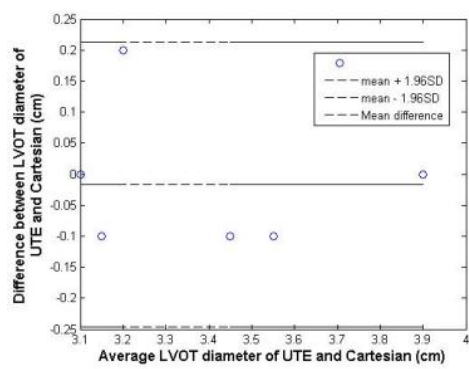

(c)

Figure 70: Bland-Altman analysis of LVOT diameter calculated from Doppler echo and 4D flow UTE MRI (a), Doppler echo and conventional 4D flow MRI (b), and conventional 4D flow MRI and 4D flow UTE MRI (c).

Figure 71 and Figure 72 demonstrate magnitude and phase velocity maps of subject 8 with porcine valve replacement using conventional and UTE technique. The conventional technique reveals a significant artifact due to susceptibility of the ferromagnetic structures
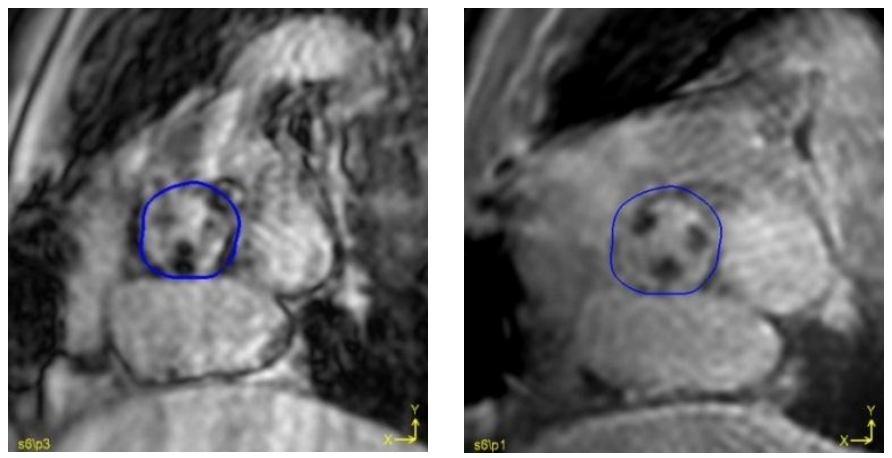

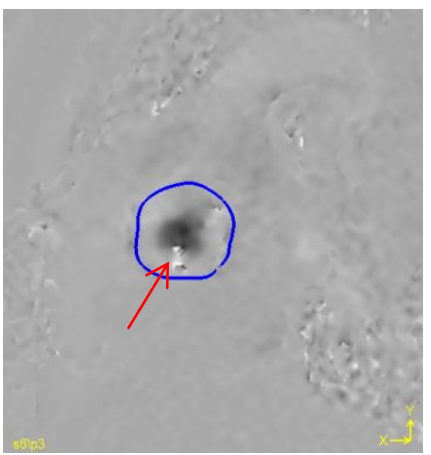

(a)

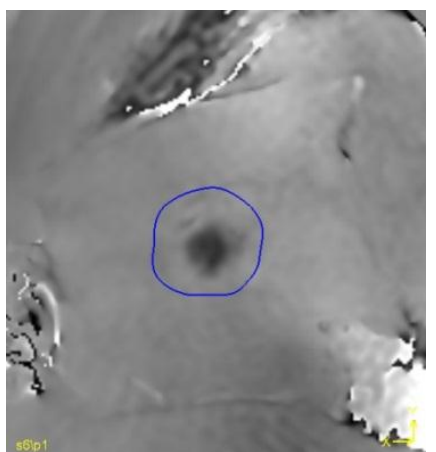

(b)

Figure 71: Magnitude and phase of conventional 4D flow MRI (a) and 4D UTE flow MRI (b) in subject 8 with porcine valve replacement at the level of the aortic valve. Susceptibility artifacts can be seen in (a) . 
in the replaced valve, leading to signal loss in the magnitude image and inaccurate peak velocity and velocity maps. In comparison, UTE reveals more uniform SNR and less artifact in the phase velocity maps.

Figure 73 shows the velocity magnitude (top left corner of each image) and velocity profiles of aortic valve in subject 4 with AS. The velocity magnitude and profiles were shown using both conventional 4D MRI and 4D UTE MRI at peak systolic time. The velocity magnitude and profile disclose a good correspondence between the conventional and UTE techniques.

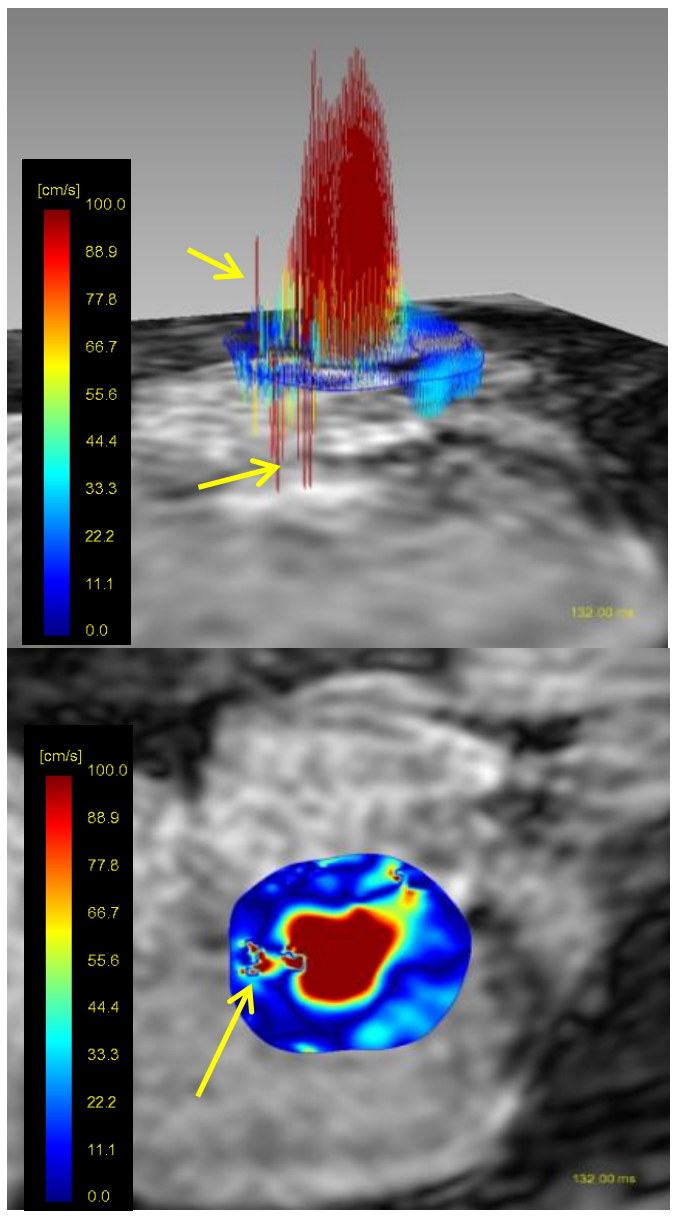

(a)

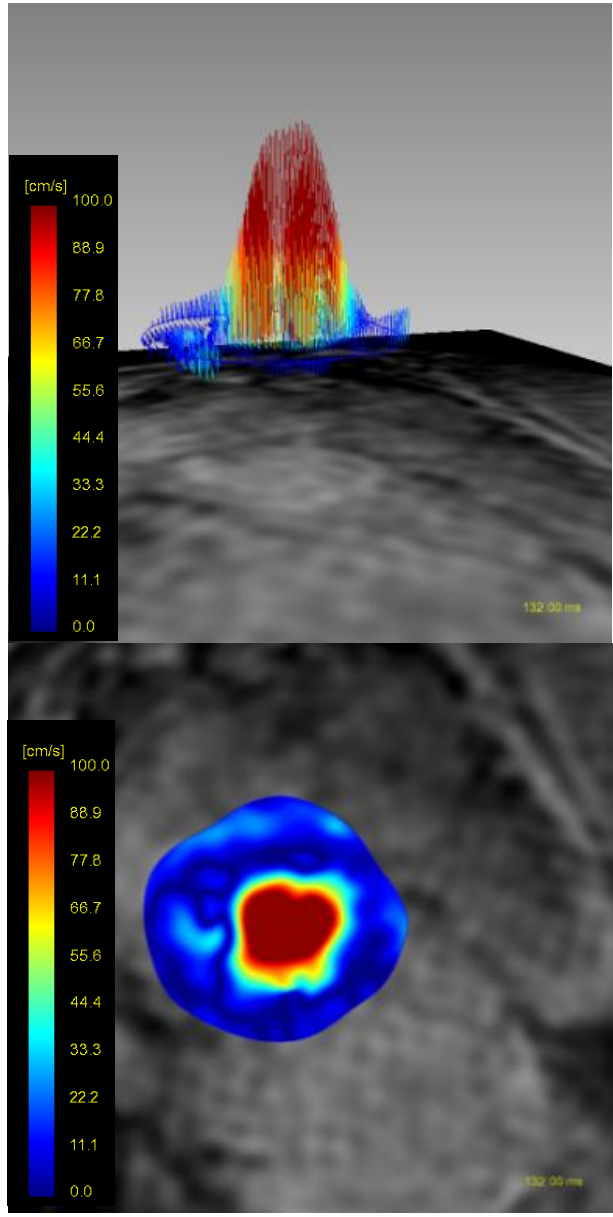

(b)

Figure 72: Velocity profile in 3D and 2D view using conventional 4D flow MRI (a) and 4D UTE flow MRI (b) in subject 8 who had porcine valve replacement at the aortic valve. Susceptibility artifacts in both views of the conventional technique is clear. 


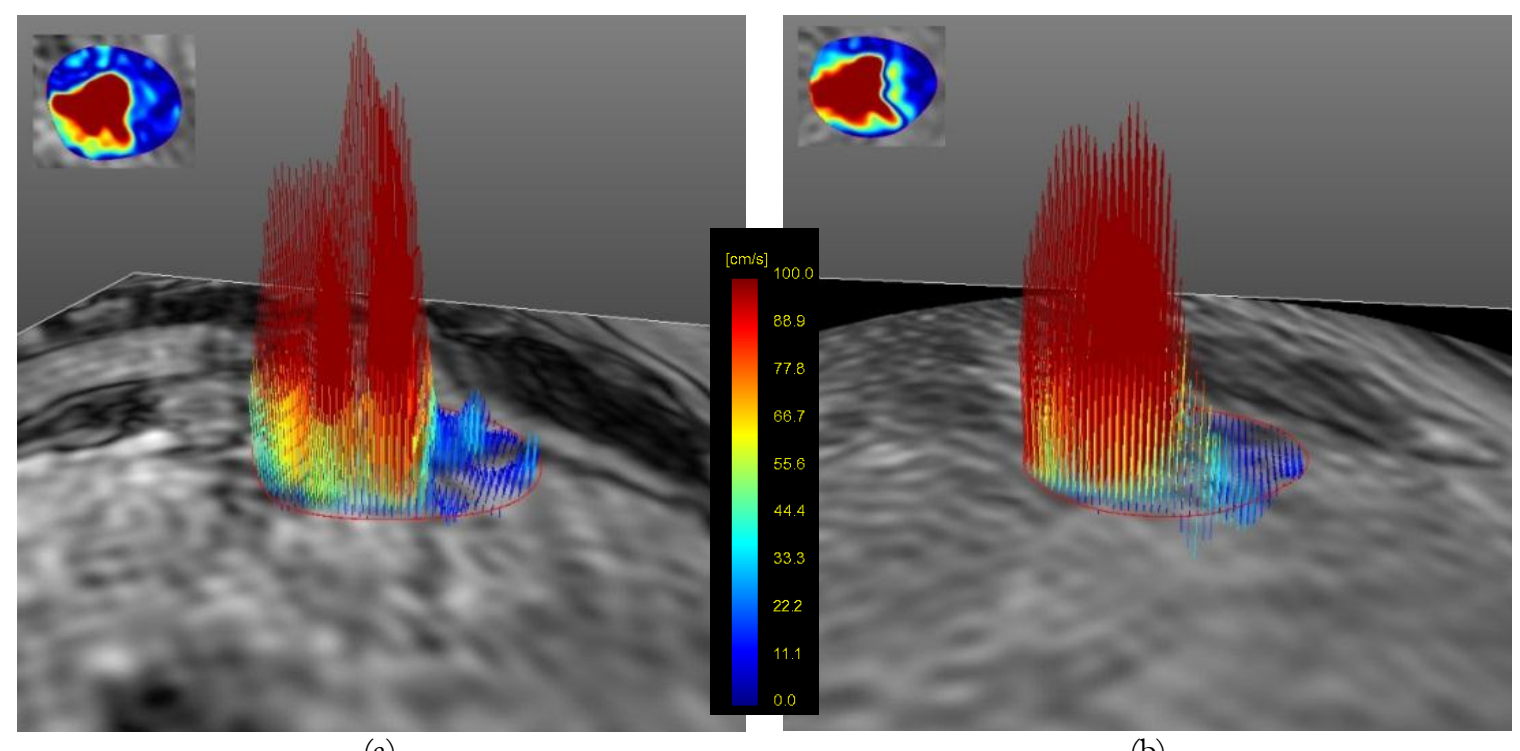

(a)

(b)

Figure 73: Velocity magnitude and profile at the level of aortic valve in subject 4 with AS at peak systolic time using conventional 4D flow (a) and 4D UTE flow (b).

Figure 74 demonstrates the velocity magnitude and velocity profiles of aortic valve in subject 5 with AR. The velocity magnitude and profiles were shown using both conventional 4D MRI and 4D UTE MRI at velocity peak time and during AR. There is good correlation between the two techniques and the regurgitation can be clearly seen in velocity profile.

Similar to previous Figure, Figure 75 shows the velocity magnitude and profiles of aortic valve in subject 6 with both AS and AR. The velocity magnitude and profiles were shown using both conventional 4D MRI and 4D UTE MRI at peak systolic time and during AR. There is a slight difference between the velocity profile and magnitude acquired using these two techniques which is mainly due to high velocity. 


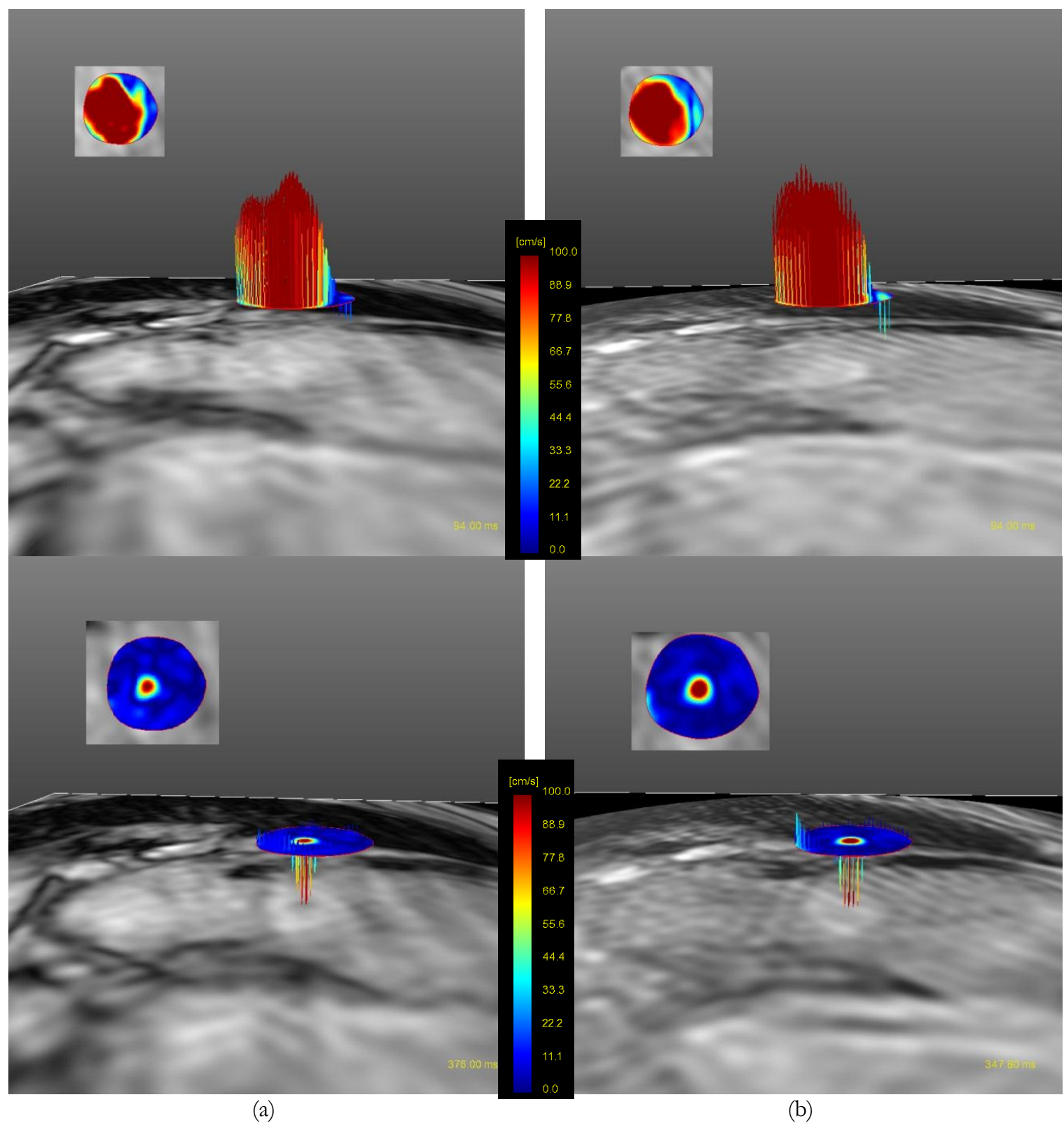

Figure 74: Velocity magnitude and profile in subject 5 with AR at peak systolic time (first row) and during AR (second row) using conventional 4D flow (a) and 4D UTE flow (b). 


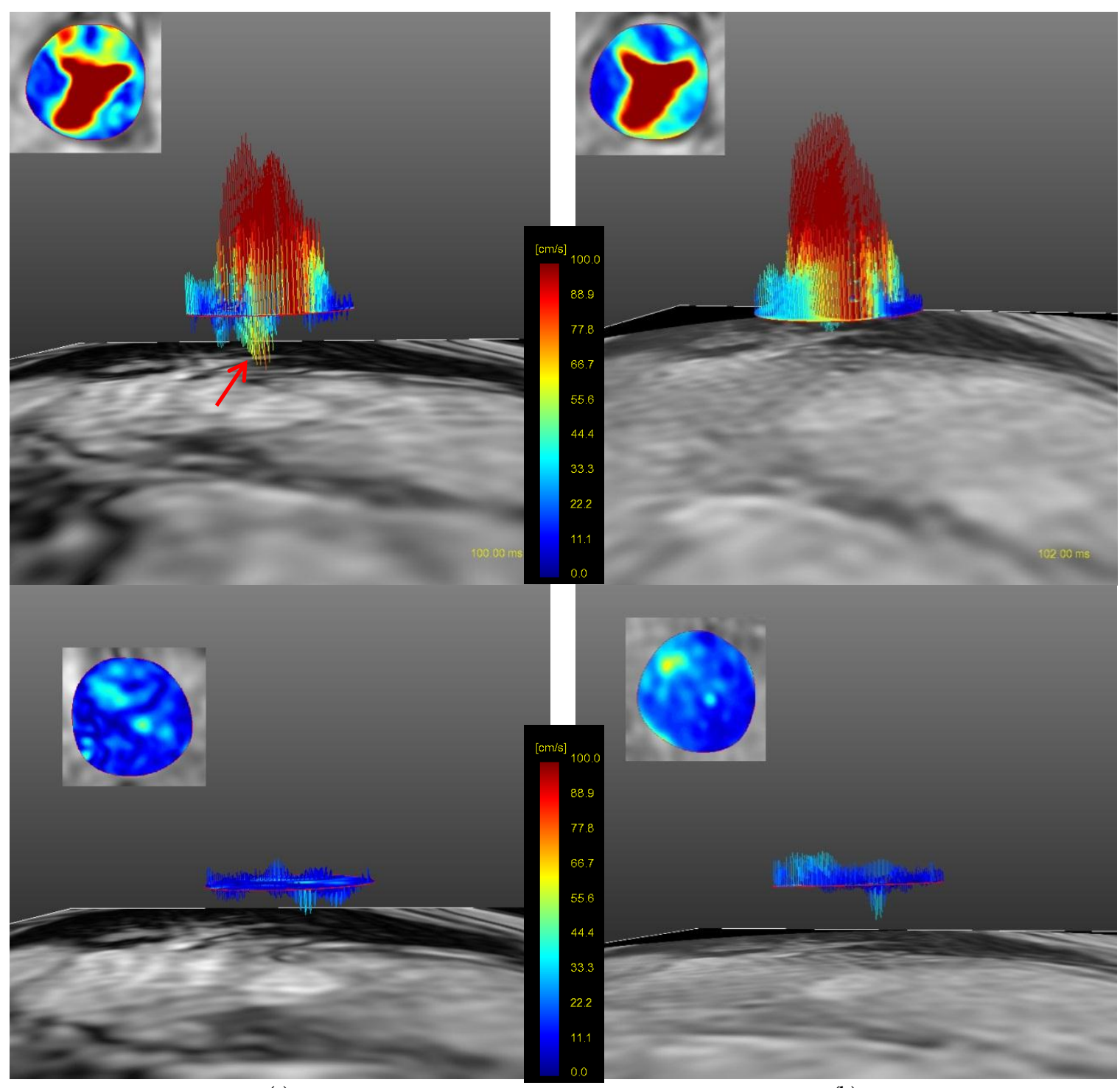

(a)

(b)

Figure 75: Velocity magnitude and profile in subject 6 with AS and AR at peak systolic time (first row) and during AR (second row) using conventional 4D flow and 4D UTE flow (b).

Figure 76 demonstrates the velocity profiles in subject 7 with AS and AR at the level of the aortic valve, using conventional 4D flow MRI (a) and 4D UTE flow MRI (b). The Figure shows a snap shot of the velocity profiles at the end-systolic time. The correlation between the two results was assessed based on the color coding of the velocity. A slight difference between flow pathlines obtained from the two sequences can be observed in locations distal 
to the valve where turbulence appears at high flow rate. Nevertheless, the correlation between the color-map and velocity profiles using the two sequences can be appreciated.

In Figure 77 and Figure 78 velocity magnitude and profiles of subjects 9 and 10 are shown at the level of the aortic valve at peak systolic time. In subject 9, the measured peak velocity using 4D UTE flow MRI shows some variations compared to conventional 4D flow MRI due to higher streaking artifact in this subject. The velocity profile and magnitude still look acceptable and are similar. The correspondence of measured velocity magnitude and profile in patient 10 is clear.

Figure 79 demonstrates the velocity magnitude and velocity profiles of aortic valve in subject 11 with AS. The velocity magnitude and profiles were shown using both conventional 4D flow and 4D UTE flow at peak systolic time. There is good correlation between the two techniques and the regurgitation can be clearly seen in velocity profile. The similarity of velocity magnitude at an axial cross section shown in the inset (the frame at top left corner of each image) between the two techniques is appreciable.

Similarly, Figure 80 demonstrates the velocity magnitude and velocity profiles of aortic valve in subject 12 with AS and again a nice correlation can be see between two techniques. The cross-sectional velocity magnitudes in the inset show the severity of stenosis and the shape of valve opening at peak systolic time. Both method yield very similar results for this subject.

One might note that, no signal loss and intavoxel dephasing are observed in aforementioned subjects - this is because the subjects recruited to the study only had mild or moderate AS. It is expected that in subjects with severe AS (velocities in the high $3 \mathrm{~m} / \mathrm{s}$ 
range and pressure difference $>40 \mathrm{~mm} \mathrm{Hg}$ ), that appreciable intravoxel dephasing would be present.

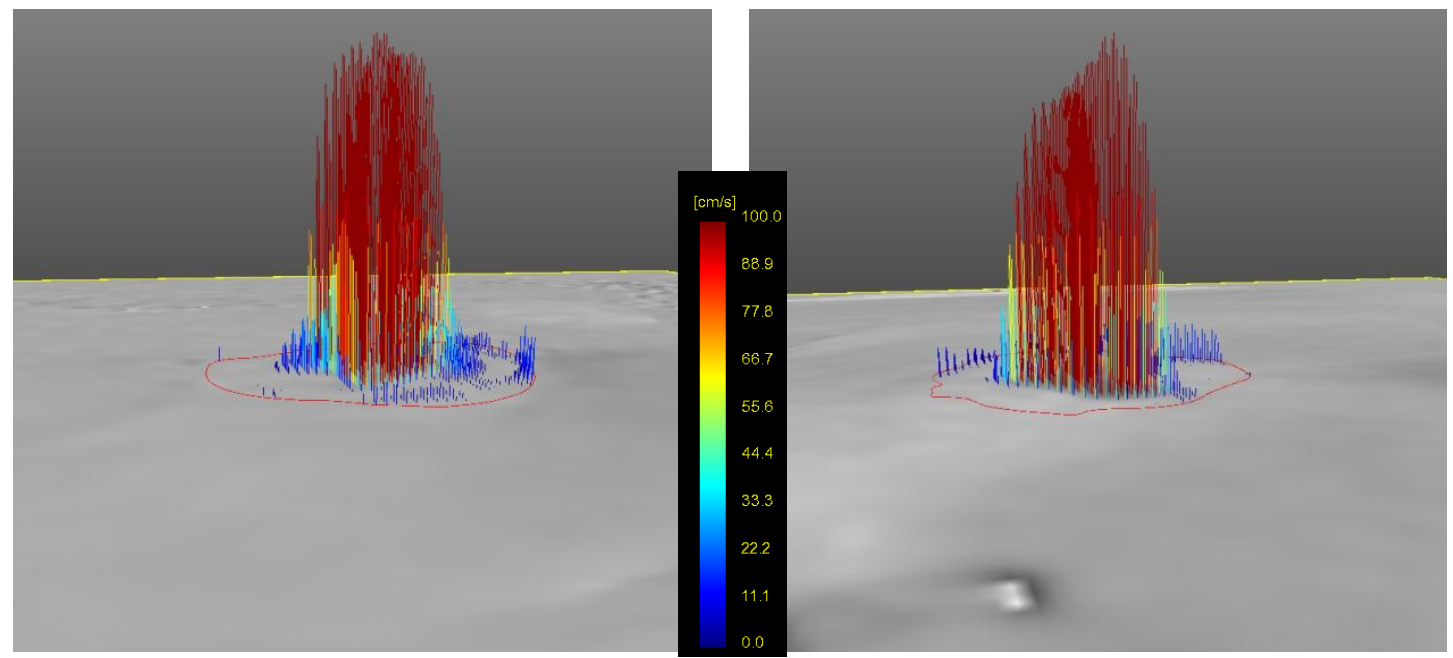

(a)

(b)

Figure 76: Velocity profile at aortic valve in subject 7 with AS and AI using conventional 4D flow MRI (a) and 4D UTE flow MRI (b). The velocity profiles reveal a good correspondence between two techniques.

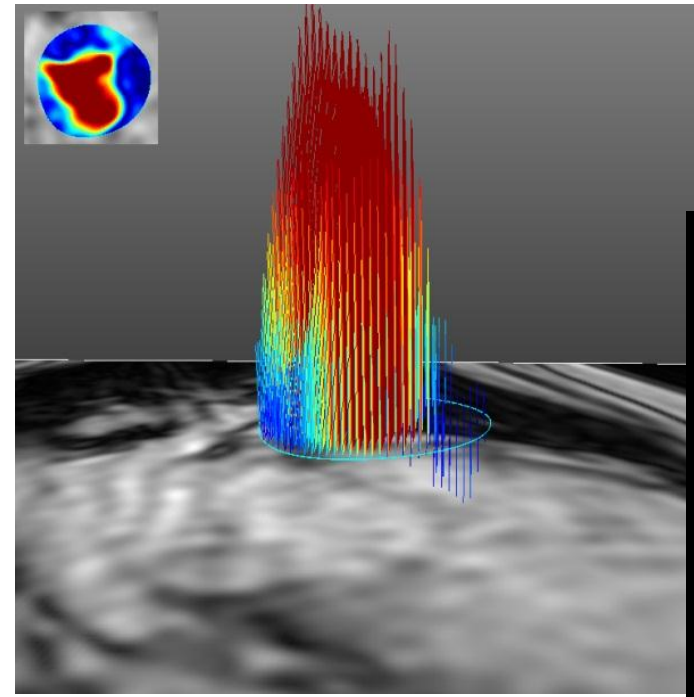

(a)

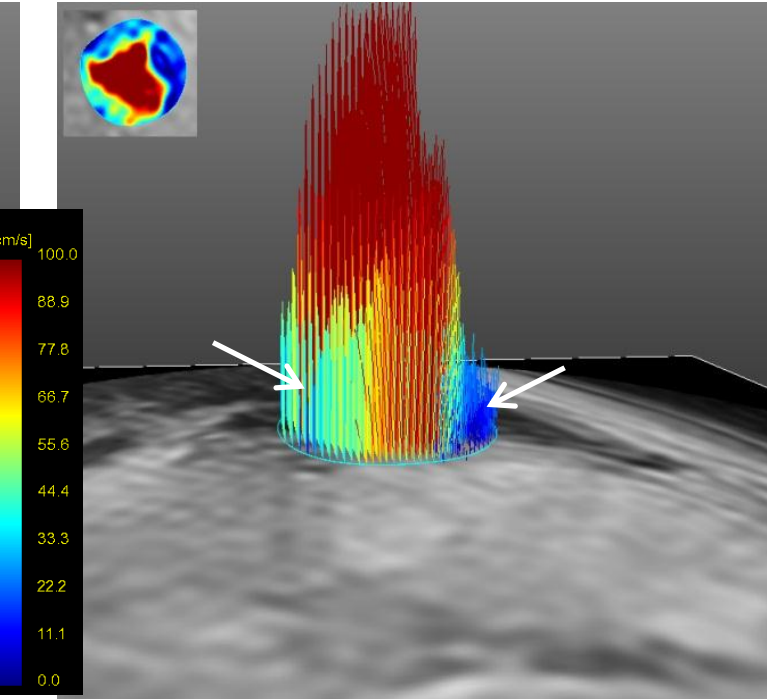

(b)

Figure 77: Velocity magnitude and profile in subject 9 with AS and AR at peak systolic time using conventional 4D flow (a) and 4D UTE flow (b). Velocity profile using 4D UTE flow MRI is different due to radial undersampling and streaking artifact which yields incorrect velocities (arrows). 


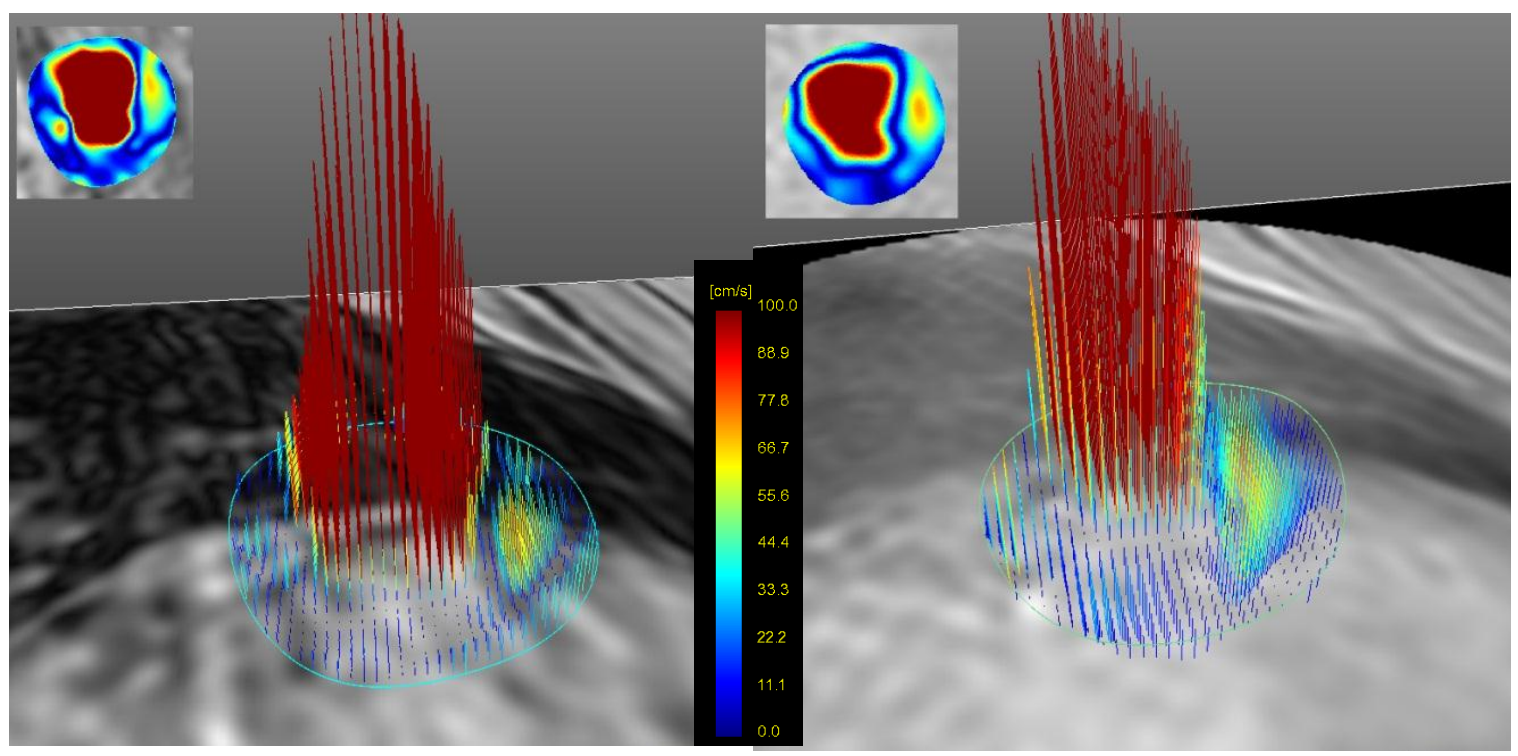

(a)

(b)

Figure 78: Velocity magnitude and profile in subject 10 with AS and AR at peak systolic time using conventional 4D flow (a) and 4D UTE flow (b). There is a good correlation between velocity magnitude and profile measured by two techniques.

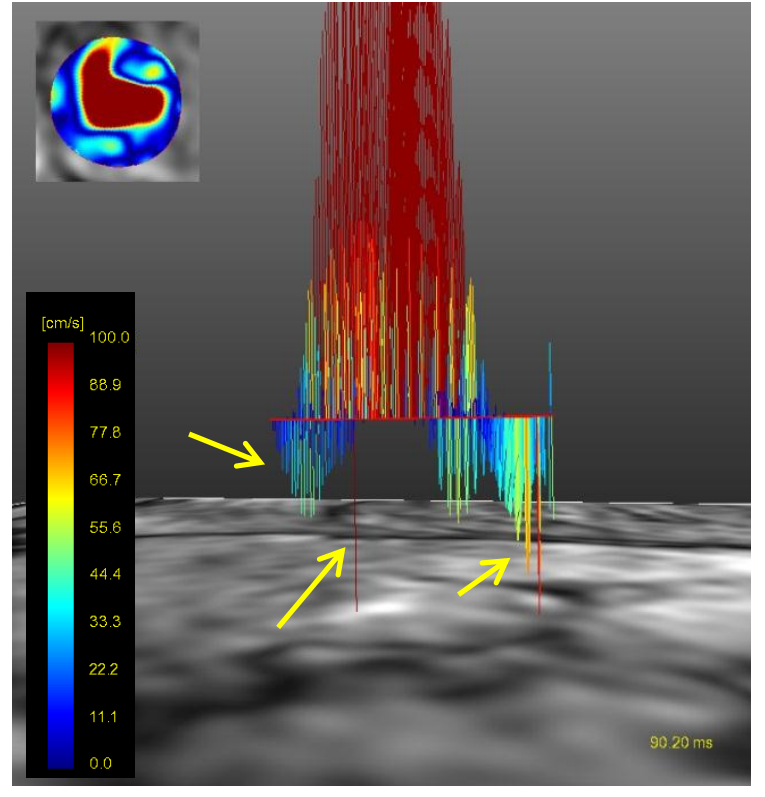

(a)

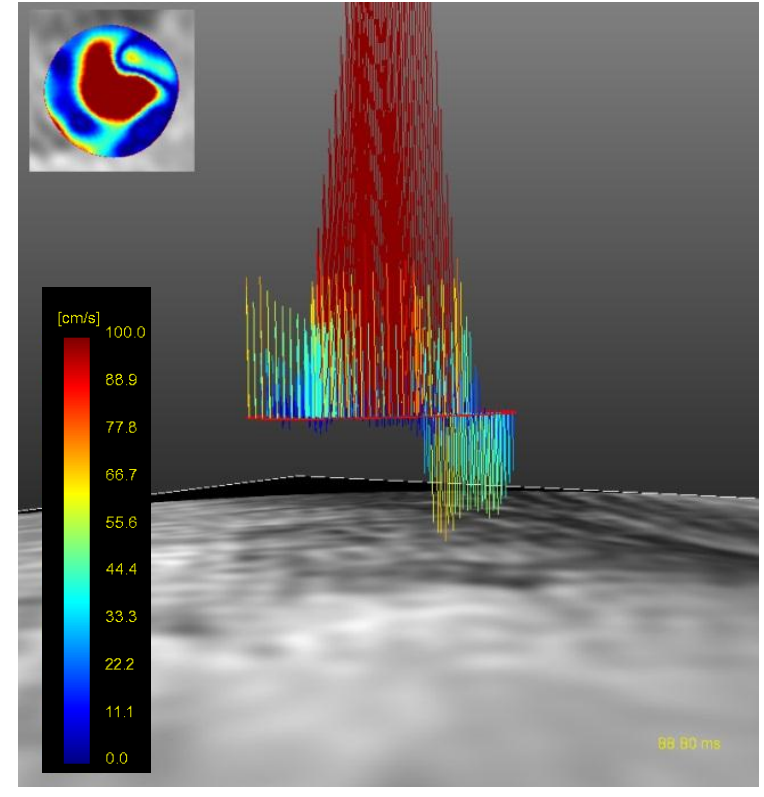

(b)

Figure 79: Velocity magnitude and profile in subject 11 with AS at peak systolic time using conventional 4D flow (a) and 4D UTE flow (b). Velocity profile using conventional 4D flow MRI shows more flow artifacts due to high velocities present. 


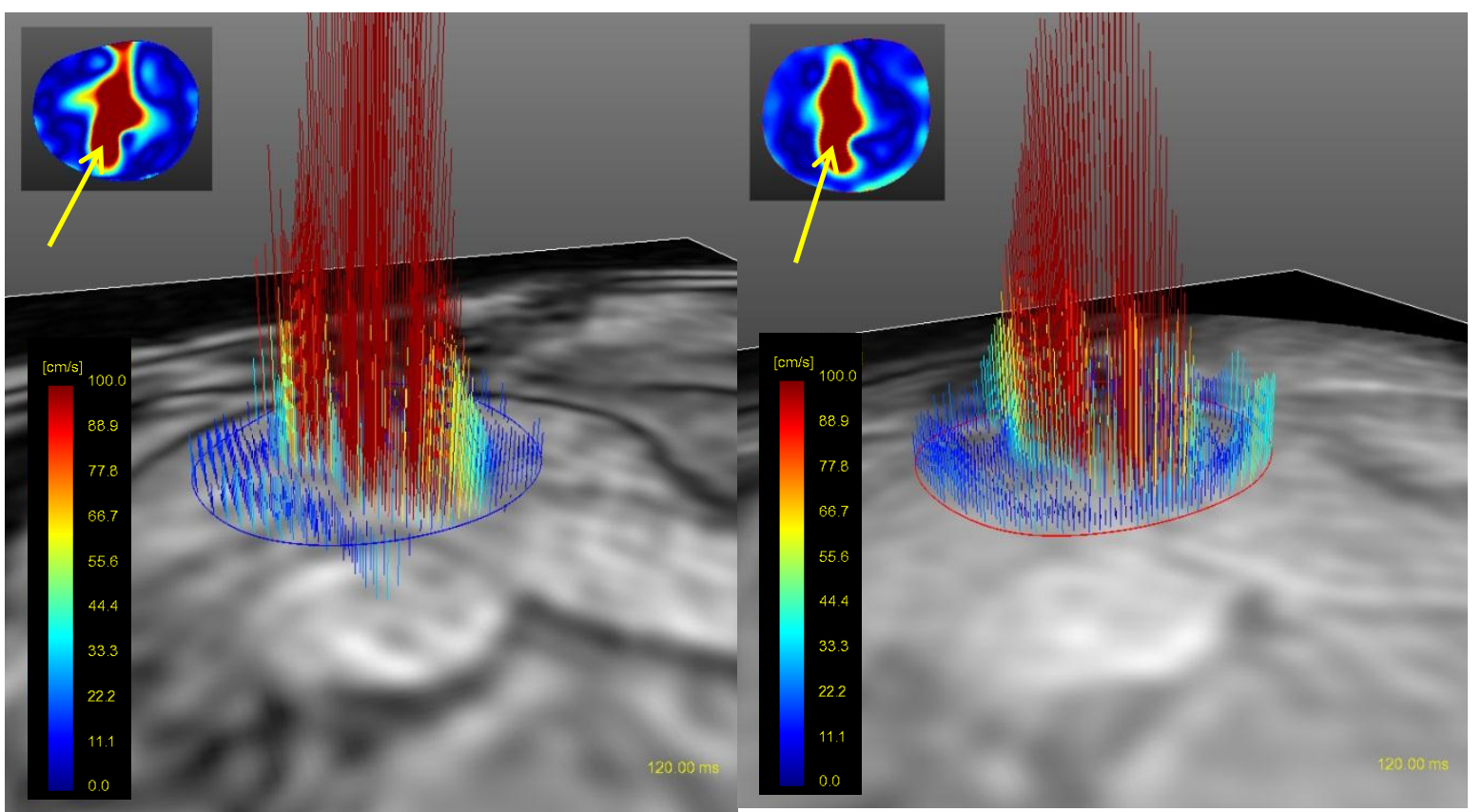

(a)

(b)

Figure 80: Velocity magnitude and profile in subject 12 with AS at peak systolic time using conventional 4D flow (a) and 4D UTE flow (b). Arrows show the similarity of two techniques in determining the shape of valve narrowing from velocity magnitude. 


\section{CONCLUSIONS AND FUTURE WORK}

\subsection{Discussion and Conclusions}

In this project, 3-D and 4-D cine UTE phase contrast MRI technique for accurate quantification of disturbed and turbulent flow have been proposed. The 3-D cine UTE-PC MRI results in reasonable flow quantification in normal volunteers with respect to conventional multi-2D cine PC MRI. The proposed 3-D sequence enables comprehensive investigation of flow in the various vascular anatomies such as carotid bifurcation and aorta 3-D acquired data provides more anatomical information as well as accurate flow quantification and characterization in the setting of vascular or valvular stenosis and/or coarctation. These pathologies can cause intravoxel dephasing and signal loss in flow images due to turbulence or disturbed flow. The achieved TE in the proposed method $0.95 \mathrm{msec}$ which is shorter than previously published TE for assessing blood in the carotid bifurcation

( $3 \mathrm{msec})$ [9]. In addition 3-D UTE-PC technique can lead to improved spatial resolution compared to 2-D technique and can reduce partial volume errors [78].

Previous phantom study on 2-D UTE-PC MRI revealed $27 \%$ underestimation of flow rate compared to the standard technique at $\mathrm{Q}=100 \mathrm{~mL} / \mathrm{s}$ (Reynolds number=7200) [12]. This error will be even more severe at lower flow rates. This is most likely due to high sensitivity of the technique to system imperfections which lead to phase errors with the UTE technique. 
In Chapter 6, stenotic phantom experiments with steady flows were reported and the accuracy of flow measurement with different $\mathrm{k}$-space trajectories and echo times were studied. The proposed 3-D UTE PC MRI technique was compared with the conventional Cartesian PC MRI technique as well as with our group's previously published spiral PC MRI method [187]. With reduced TE, the spiral acquisition resulted in reduced signal loss and phase error in the magnitude and the velocity image. However, in comparison to the 3-D UTE acquisition, a slight signal loss in the magnitude was still observable in the high flow rate $\mathrm{Q}=300 \mathrm{ml} / \mathrm{s}$ study. 3-D UTE PC MRI resolved the signal loss and phase error entirely in the setting of high flow rate. In addition, 3-D UTE-PC MRI techniques exhibits less variability in flow quantification in various flow rates compared to Conventional PC MRI technique.

It wasshown that in contrast with previously published 2-D UTE sequence [12], the accuracy of proposed 3-D UTE technique is comparable with standard PC MRI technique in the setting of flow rates as low as $13.2 \mathrm{~mL} / \mathrm{s}$ (Reynolds number=160). This improvement is likely due to the phase correction step as well as less phase error due to eddy current in proposed 3-D UTE technique. Further, it was shown the accuracy of flow measurement is higher even for the case of low flow rates in carotid arteries.

Unlike conventional 2-D UTE MRI techniques, the current method can be tailored to significantly reduce the slice thickness, making it a robust technique applicable to different clinical applications. In addition, by resolving the dependency of slice thickness and flow encoding gradients of previous UTE techniques, determination of components of in-plane flow will become feasible. 
In Chapter 7, a comprehensive assessment of parameter variation in carotid artery using 3D UTE PC MRI in 3T scanner was presented. The effect of accurate Venc selection in accuracy of flow measurement was discussed. A reasonable compromise between scan time and accuracy of flow quantification was achieved using 50-75\% sampling of k-space lines. In addition, TE was reduced to $1 \mathrm{msec}$ using L5 gradient strength for Venc $=150$. Higher gradient strength and slew rates result in more eddy current, causing increased phase error and affect the accuracy of flow quantification. Eddy current is more severe in case of low Venc wherein the gradient strength needs to be set to higher amplitude to achieve a lower Venc. Improvement of phase error correction for more accurate flow quantification in case of lower Venc will be investigated. In addition, the effect of various gradient field strengths and slew rates on flow quantification will be investigated using the flow phantom in high flow rates and in-vivo study in the case of high flow rates.

The 3D UTE PC MRI, and more generally all 3D PC MRI techniques, collect flow and velocity information in only a single direction. In order to get comprehensive flow information in all three directions from 3D PC MRI, the scan needs to be repeated three times. 4D flow MRI, on the other hand, acquires flow data in all three directions in a single scan. However, this single scan generally is long. In Chapter 8, a reduced TE 4D spiral flow technique was implemented with the goal of reducing the scan time compared to conventional 4D PC MRI while resulting in reduced TE compared to previously published techniques. The accuracy of flow quantification and characterization with the spiral acquisition revealed a good agreement with the conventional technique. The systolic mean velocity, peak flow and the average flow in CCA and ICA of normal volunteers using 4D spiral flow MRI showed errors less than 10\% compared to conventional 4D flow. In addition, the scan using the spiral sequence took 3:31 minutes which is half of the time of 
the conventional sequence. Although the TE in 4D spiral flow MRI was reduced in comparison to conventional 4D flow MRI, however, it is felt that the reduction is not significant enough to resolve the intravoxel dephasing which can occur in the case of high flow rates.

In Chapter 9, a novel 4D UTE flow MRI technique was presented which benefits from a significantly shorter TE relative to conventional 4D flow MRI. The phantom measurement for the steady and pulsatile flow regime with various flow rates revealed good correlation between measured flow from conventional and UTE sequences at low and medium flow rates. At high flow rates, however, the conventional sequence resulted in intravoxel dephasing and signal loss causing flow related artifact in velocity contours. Compared to the conventional sequence, the UTE sequence performs more accurate flow quantification and flow waveform results for the UTE sequence reveal significant. In summary, the phantom studies performed with 4D UTE flow show the potential of this technique for more accurate assessment of stenotic flows by mitigating intravoxel dephasing and signal loss, expanding the clinical applicability of 4D flow MRI.

In Chapter 10, it was shown that MRI can be used to measure flow and other parameters for a stenotic aortic valve. A good correlation was observed between MRI and Doppler echo for area measurement at the aortic valve. Patients with moderately severe Aortic Stenosis (AS), including Aortic Regurgitation (AR) and insufficiency (AI) were studied using conventional 4D flow and 4D UTE flow. In both cases, the result of peak velocity, LVOT diameter, AV peak time, and AV eject time had close correspondence with Doppler echo.

Table 14 compares different flow imaging techniques which were used in this dissertation. For the purposes of this comparison, it was assumed that the UTE techniques 
has $75 \% \mathrm{k}$-space sampling of radial k-space line while the spiral technique uses 4 msec readouts and 30 interleaves. In general, the shortest scan times are achieved when 3D spiral sequence acquires flow velocities in a single direction. The longest scan times result when the 4D UTE sequence acquires flow in three flow directions. The scan time for UTE techniques with $50 \%$ sampling of $\mathrm{k}$-space lines is comparable with the conventional techniques. The shortest TE was achieved when using 3D and 4D UTE techniques resulting in least flow artifacts. However, significant streaking artifact occurs due to k-space radial under-sampling. Spiral techniques reduce the flow artifact (but not completely) compared to conventional techniques while they have the shortest scan time. The TE in this technique is shorter than conventional and longer than UTE. Other possible artifacts for different methods which are reported in the Table are further explained here. Fold over artifact is a common artifact in

Table 14: Comparison between different MR flow measurement techniques utilized or developed in this dissertation. All the imaging parameters were considered the same at flow rate $300 \mathrm{ml} / \mathrm{s}$ which required a Venc of 500 which is assumed the same for all methods and for all 3 directions. The UTE techniques have $75 \%$ sampling rate of radial $\mathrm{k}$-space line and the spiral techniques used $4 \mathrm{msec}$ readouts and 30 interleaves. \#Encoding in the table refers to number of flow encoding directions during one scan.

\begin{tabular}{|c|c|c|c|c|c|}
\hline Techniques & $\begin{array}{l}\text { Scan time ( } \\
\text { minute) }\end{array}$ & $\begin{array}{c}\text { TE } \\
\text { (msec) }\end{array}$ & Flow artifact & Other artifacts & $\begin{array}{c}\# \\
\text { Encoding }\end{array}$ \\
\hline Conventional 3D & 3 & 3.4 & high & Fold over- signal loss & 1 \\
\hline $\begin{array}{c}\text { 3D Spiral } \\
30 \text { interleaves }\end{array}$ & 2 & 1.5 & moderate & $\begin{array}{l}\text { Off resonance- slight } \\
\text { signal loss }\end{array}$ & 1 \\
\hline $\begin{array}{c}\text { 3D UTE } \\
75 \% \text { radial k-space } \\
\text { sampling }\end{array}$ & $3: 30$ & 0.95 & low & $\begin{array}{c}\text { Streaking artifact- } \\
\text { eddy current }\end{array}$ & 1 \\
\hline Conventional 4D & 12 & 3.9 & high & Fold over- signal loss & 3 \\
\hline $\begin{array}{c}\text { 4D Spiral } \\
30 \text { interleaves }\end{array}$ & 6 & 1.6 & moderate & $\begin{array}{c}\text { Off resonance- slight } \\
\text { signal loss }\end{array}$ & 3 \\
\hline $\begin{array}{c}\text { 4D UTE } \\
75 \% \text { radial k-space } \\
\text { sampling }\end{array}$ & 13 & 0.98 & low & $\begin{array}{l}\text { Significant Streaking } \\
\text { artifact- eddy current }\end{array}$ & 3 \\
\hline
\end{tabular}


conventional technique in which undesired object from outside of FOV may be incorrectly mapped to the location inside the FOV. UTE and spiral methods do not suffer from this artifact in the in-plane directions though if phase-encoding is performed in the $z$ direction, fold over may be present in that direction (e,g, with stack of stars or stack of spirals). On the other hand, off-resonance artifact occurs in spiral technique in case of long readouts and smaller number of interleaves. However, spiral readouts have the benefit of no ghosting artifacts which may occur due to vessel pulsation.

Eddy current artifact arises when the gradient strength is high or the gradient switching is very fast. This is mainly problematic when gradient performance is pushed to the limit to shorten TE.

\subsection{Future work}

One of the main limitations of $4 \mathrm{D}$ flow MRI is long acquisition time which is more problematic when performing in-vivo studies. Long acquisitions for in-vivo studies lead to the need to reduce volume coverage and/or spatial and temporal resolutions. Although the scan time for 4D UTE flow is in general longer than conventional 4D flow, one may reduce the total scan time in 4D UTE flow through radial under-sampling of k-space though at the cost of reduced accuracy. For the flow rates considered, it was demonstrated that 25\% sampling of $\mathrm{k}$-space results in a considerable error whereas $50 \%$ sampling of $\mathrm{k}$-space still results in acceptable accuracy. For the phantom studies performed, 50\% sampling resulted in acceptable scan times while $75 \%$ sampling resulted in higher accuracy and slightly longer scan time when compared to conventional 4D flow. To further reduce the scan time, parallel 
imaging techniques such as SENSE [188] and GRAPPA [189], k-t BLAST/SENSE [190], or compressive sampling [191] may be used in conjunction with 4D UTE flow. As an alternative method, 4D spiral flow MRI can result in shorter scan time compared to conventional 4D flow MRI and 4D UTE flow MRI and may have significant utility when it is known that high flow rates and intravoxel dephasing do not occur. Further investigation to study the accuracy of 4D spiral flow MRI using the stenotic flow phantom will be helpful.

A second area for further investigation may be to employ retrospective gating, as prospective gating usually leads to missing information during end-diastole and therefore flow measurement errors. Retrospective gating can collect flow images throughout the cardiac cycle while improving the temporal resolution.

One limitation of the UTE technique is the higher sensitivity of the technique to scanner hardware imperfection including gradient delay and field inhomogeneity. Although, in this study, phase error and gradient delay correction techniques were employed, still some erroneous streamlines attributable to trajectory errors could be observed - please see Figure 58(b) close to the wall (where streamlines start from the wall and move inwards). This error is mainly due incomplete correction of gradient delay and which results in a phase error. RF field inhomogeneity needs to be additionally considered for the UTE technique which is more problematic in larger FOV's and off-center of large volumes. In our study, Figure 49 illustrates the effect of RF field inhomogeneity at off-center locations which clearly result in phase errors and velocity miscalculations, requiring an additional correction. RF field inhomogeneity can be reduced by using parallel RF transmit in higher magnetic field strength. 
For the present phantom study, in addition to the scanner hardware imperfections, the effect of pump errors should also be considered - these are primarily due to the pump tolerance and mechanical effects; though transient bubbles may also contribute to this class of measurement errors.

The result of patient studies on moderately severe AS subjects did not reveal significant difference between conventional 4D flow MRI and 4D UTE flow MRI since the peak velocities distal to the valve in these subjects were not high enough to lead to turbulent flow and significant spin dephasing. In patients with severe or critical AS, on the other hand, the peak velocity is expected to be much higher, resulting in introvoxel dephasing and artifacts in conventional 4D flow. Further investigations in patients with severe or critical AS will be necessary. 


\section{REFERENCES}

1. Hope, T.A. and R.J. Herfkens, Imaging of the thoracic aorta with time-resolved threedimensional phase-contrast MRI: a review. Semin Thorac Cardiovasc Surg, 2008. 20(4): p. 358-64.

2. Gatehouse, P.D., et al., Applications of phase-contrast flow and velocity imaging in cardiovascular MRI. Eur Radiol, 2005. 15(10): p. 2172-84.

3. Evans, A.J., et al., Effects of turbulence on signal intensity in gradient echo images. Invest Radiol, 1988. 23(7): p. 512-8.

4. Hamilton, C.A., et al., Effects of intravoxel velocity distributions on the accuracy of the phase-mapping method in phase-contrast MR angiography. J Magn Reson Imaging, 1994. 4(5): p. 752-5.

5. Nayak, K.S., B.S. Hu, and D.G. Nishimura, Rapid quantitation of high-speed flow jets. Magn Reson Med, 2003. 50(2): p. 366-72.

6. Sondergaard, L., et al., Valve area and cardiac output in aortic stenosis: quantification by magnetic resonance velocity mapping. Am Heart J, 1993. 126(5): p. 1156-64.

7. Sondergaard, L., et al., Accuracy and precision of MR velocity mapping in measurement of stenotic cross-sectional area, flow rate, and pressure gradient. J Magn Reson Imaging, 1993. 3(2): p. 433-7.

8. Stahlberg, F., et al., Pulse sequence design for $M R$ velocity mapping of complex flow: notes on the necessity of low echo times. Magn Reson Imaging, 1994. 12(8): p. 1255-62.

9. O'Brien, K.R., et al., MRI phase contrast velocity and flow errors in turbulent stenotic jets. J Magn Reson Imaging, 2008. 28(1): p. 210-8.

10. Stahlberg, F., et al., Quantification of complex flow using MR phase imaging--a study of parameters influencing the phase/velocity relation. Magn Reson Imaging, 1992. 10(1): p. 13-23.

11. Schmalbrock, P., et al., Volume MR angiography: methods to achieve very short echo times. Radiology, 1990. 175(3): p. 861-5.

12. O'Brien, K.R., et al., Phase contrast ultrashort TE: A more reliable technique for measurement of high-velocity turbulent stenotic jets. Magn Reson Med, 2009. 62(3): p. 626-36.

13. Kadbi, M., Negahdar, M., Cha, J.W., Traughber, M., Martin, P., Stoddard, M., Amini, A.A., 4D UTE Flow: A Phase-Contrast MRI Technique for Assessment and Visualization of Stenotic Flows. Magn Reson Med, under revision, 2013.

14. Guyton, A.C.H., J.E., Textbook of Medical Physiology. 11 ed. 2006, Philadelphia: Elsevier Saunder.

15. DN, K., Blood flow in arteries. Annual Review of Fluid Mechanics 1997. 29: p. 399-434.

16. G., C.C., The Mechanics of the Circulation. 1978: Oxford University Press.

17. http://en.wikipedia.org/wiki/Ascending aorta.

18. Homaeinezhad, M.R.G., A. ; Aghaee, M.; Toosi, H. N.;Rahmani, R. , A high-speed C++/MEX solution for long-duration arterial blood pressure characteristic locations detection. Biomedical Signal Processing and Control, 2012. 7(2): p. 151-172. 
19. Seeley, R.J., Anatomy and physiology. 1992: McGraw-Hill Companies.

20. Blacher, J., et al., Aortic pulse wave velocity as a marker of cardiovascular risk in hypertensive patients. Hypertension, 1999. 33(5): p. 1111-7.

21. Manning, W.J., Asymptomatic aortic stenosis in the elderly: a clinical review. JAMA, 2013. 310(14): p. 1490-7.

22. http://www.texasheartinstitute.org/HIC/Topics/Cond/CarotidArteryDisease.cfm.

23. Gray, H., Gray's Anatomy 2004: Churchill Livingstone.

24. http://www.lab.anhb.uwa.edu.au/mb140/corepages/vascular/vascular.htm.

25. Scott, E.M., Cardiovascular Physiology: An Integrative Approach. 1986: Manchester Univ Pr.

26. Libby, P., Inflammation in atherosclerosis. Nature, 2002. 420(6917): p. 868-74.

27. Ross, R., Atherosclerosis--an inflammatory disease. N Engl J Med, 1999. 340(2): p. 11526.

28. http://www.emedicinehealth.com/script/main/art.asp?articlekey=127987\&ref $=126544$.

29. Klabunde, R.E., Cardiovascular Physiology Concepts. 2004: Lippincott Williams \& Wilkins. 30. http://me.queensu.ca/People/Sellens/PowerLaw.html.

31. White, F.M., Viscous Fluid Flow. 3rd ed. 2005: McGraw-Hill Science/Engineering/Math.

32. Womersley, J.R., Method for the calculation of velocity, rate of flow and viscous drag in arteries when the pressure gradient is known. J Physiol, 1955. 127(3): p. 553-63.

33. $\mathrm{O}, \mathrm{R} .$, An experimental investigation of the circumstances which determine whether the motion of water shall be direct or sinuous, and of the law of resistance in parallel channels. Philosophical Transactions of the Royal Society of London, 1883. 174: p. 935982.

34. Westerhof, N.S., N.; Noble, M. I. M., Snapshots of hemodynamics. 1st ed. 2005, New York: Springer.

35. Avila, K., et al., The onset of turbulence in pipe flow. Science, 2011. 333(6039): p. 192-6.

36. Farzan, G., Low Reynolds number turbulence modeling of blood flow in arterial stenoses. Biorheology, 1998. 35: p. 281-294.

37. Stein, P.D. and H.N. Sabbah, Turbulent blood flow in the ascending aorta of humans with normal and diseased aortic valves. Circ Res, 1976. 39(1): p. 58-65.

38. Smith, R.L., et al., Thrombus production by turbulence. J Appl Physiol, 1972. 32(2): p. 261-4.

39. Roberts, W.C., M.C. Fishbein, and A. Golden, Cardiac pathology after valve replacement by disc prosthesis. A study of 61 necropsy patients. Am J Cardiol, 1975. 35(5): p. 740-60.

40. Yellin, E.L., Hydraulic noise in submerged and bounded liquid jets In Biomedical Fluid Mechanics Symposium. American Society of Mechanical Engineers, 1966: p. 209-221.

41. Roberts, W.C., B.H. Bulkley, and A.G. Morrow, Pathologic anatomy of cardiac valve replacement: a study of 224 necropsy patients. Prog Cardiovasc Dis, 1973. 15(6): p. 53987.

42. Davies, P.F., et al., Turbulent fluid shear stress induces vascular endothelial cell turnover in vitro. Proc Natl Acad Sci U S A, 1986. 83(7): p. 2114-7.

43. Yoganathan, A.P., et al., Review of hydrodynamic principles for the cardiologist: applications to the study of blood flow and jets by imaging techniques. J Am Coll Cardiol, 1988. 12(5): p. 1344-53.

44. Sabbah, H.N. and P.D. Stein, Turbulent blood flow in humans: its primary role in the production of ejection murmurs. Circ Res, 1976. 38(6): p. 513-25.

45. Nygaard, H., et al., Turbulent stresses downstream of three mechanical aortic valve prostheses in human beings. J Thorac Cardiovasc Surg, 1994. 107(2): p. 438-46. 
46. Isaaz, K., et al., Noninvasive quantitation of blood flow turbulence in patients with aortic valve disease using online digital computer analysis of Doppler velocity data. J Am Soc Echocardiogr, 2003. 16(9): p. 965-74.

47. Oshinski, J.N., D.N. Ku, and R.I. Pettigrew, Turbulent fluctuation velocity: the most significant determinant of signal loss in stenotic vessels. Magn Reson Med, 1995. 33(2): p. 193-9.

48. Xiang, Q.S. and O. Nalcioglu, Measurement of mean and variance of velocity fields within each voxel by NMR imaging. IEEE Trans Med Imaging, 1988. 7(4): p. 364-7.

49. Kuethe, D.O., Measuring distributions of diffusivity in turbulent fluids with magneticresonance imaging. Phys Rev A, 1989. 40(8): p. 4542-4551.

50. Gao, J.H. and J.O. Gore, Turbulent flow effects on NMR imaging: measurement of turbulent intensity. Med Phys, 1991. 18(5): p. 1045-51.

51. Gatenby, J.C. and J.C. Gore, Mapping of turbulent intensity by magnetic resonance imaging. J Magn Reson B, 1994. 104(2): p. 119-26.

52. Kuethe, D.O. and J.H. Gao, NMR signal loss from turbulence: Models of time dependence compared with data. Phys Rev E Stat Phys Plasmas Fluids Relat Interdiscip Topics, 1995. 51(4): p. 3252-3262.

53. Pipe, J.G., A simple measure of flow disorder and wall shear stress in phase contrast MRI. Magn Reson Med, 2003. 49(3): p. 543-50.

54. Haghi, D., et al., A hybrid approach for quantification of aortic valve stenosis using cardiac magnetic resonance imaging and echocardiography: comparison to right heart catheterization and standard echocardiography. Clin Res Cardiol, 2006. 95(3): p. 162-7.

55. Podolak, M.J., et al., Evaluation of flow through simulated vascular stenoses with gradient echo magnetic resonance imaging. Invest Radiol, 1989. 24(3): p. 184-9.

56. Rittgers, S.E., et al., Velocity profiles in stenosed models using magnetic resonance imaging. Monogr Atheroscler, 1990. 15: p. 43-53.

57. Valk, P.E., et al., MRI of blood flow: correlation of image appearance with spin-echo phase shift and signal intensity. AJR Am J Roentgenol, 1986. 146(5): p. 931-9.

58. Puddephat, M.J., Computer Interface for Convenient Application for Stereological Methods for Unbiased Estimation of Volume and Surface Area: Studies Using MRI with Particular Reference to the Human Brain. 1999, University of Liverpool.

59. Haacke, E.M.b., M. W.; Thompson, M. R.; Venkatesan, R., Magnetic Resonance Imaging: Physical Principles and Sequence Design. 1995: John Wiley \& Sons.

60. Liang, Z.L., P. C., Principles of Magnetic Resonance Imaging: A Signal Processing Perspective 1999: Wiley-IEEE Press.

61. Ulaby, F., Fundamentals of applied electromagnetics. 2007: Pearson:Prentice Hall.

62. Brown, M.A.S., R. C., MRI: Basic Principles and Applications, Mark A. Brown. 3 ed. 2003: Wiley-Liss.

63. Bloch, F., Nuclear Induction. Physical Review 1946. 70: p. 460-473

64. Pooley, A., Fundamental Physics of MR Imaging. Radiographics, 2005. 14: p. 1087-1099.

65. Bernstein, M.A.K., K. F.; Zhou, X. J., Handbook of MRI Pulse Sequences. 2004: Elsevier Inc.

66. Slavin, G.S. and D.A. Bluemke, Spatial and temporal resolution in cardiovascular MR imaging: review and recommendations. Radiology, 2005. 234(2): p. 330-8.

67. Lauterburg, P.C., Image Formation by Induced Local Interactions: Examples Employing Nuclear Magnetic Resonance. Nature, 1973. 242 p. 190 - 191.

68. Glover, G.H. and J.M. Pauly, Projection reconstruction techniques for reduction of motion effects in MRI. Magn Reson Med, 1992. 28(2): p. 275-89. 
69. Bergin, C.J., G.H. Glover, and J.M. Pauly, Lung parenchyma: magnetic susceptibility in MR imaging. Radiology, 1991. 180(3): p. 845-8.

70. Bergin, C.J., et al., MR imaging of lung parenchyma: a solution to susceptibility. Radiology, 1992. 183(3): p. 673-6.

71. Gold, G.E., et al., MR spectroscopic imaging of collagen: tendons and knee menisci. Magn Reson Med, 1995. 34(5): p. 647-54.

72. Pauly, J.C., S.; , Slice-selective excitation for very short T2 species. In Proceedings of the 8th Annual Meeting of ISMRM, 1989: p. 28.

73. Gold, G.E., et al., Characterization of atherosclerosis with a 1.5-T imaging system. J Magn Reson Imaging, 1993. 3(2): p. 399-407.

74. Gatehouse, P.D. and G.M. Bydder, Magnetic resonance imaging of short T2 components in tissue. Clin Radiol, 2003. 58(1): p. 1-19.

75. Robson, M.D., et al., Magnetic resonance: an introduction to ultrashort TE (UTE) imaging. J Comput Assist Tomogr, 2003. 27(6): p. 825-46.

76. Clow, H.P., W. S., Non-linear sampling in NMR. 1978: US.

77. Barger, A.V., et al., Time-resolved contrast-enhanced imaging with isotropic resolution and broad coverage using an undersampled 3D projection trajectory. Magn Reson Med, 2002. 48(2): p. 297-305.

78. Rahmer, J., et al., Three-dimensional radial ultrashort echo-time imaging with T2 adapted sampling. Magn Reson Med, 2006. 55(5): p. 1075-82.

79. Glover, G.H., J.M. Pauly, and K.M. Bradshaw, Boron-11 imaging with a three-dimensional reconstruction method. J Magn Reson Imaging, 1992. 2(1): p. 47-52.

80. Wu, Y., et al., Density of organic matrix of native mineralized bone measured by waterand fat-suppressed proton projection MRI. Magn Reson Med, 2003. 50(1): p. 59-68.

81. Brittain, J.H., Ultrashort TE imaging with single-digit (8 US) TE. In: Proceedings of the 12th Annual Meeting of ISMRM,Kyoto, Japan, 2004: p. 629.

82. Nielles-Vallespin, S., et al., 3D radial projection technique with ultrashort echo times for sodium MRI: clinical applications in human brain and skeletal muscle. Magn Reson Med, 2007. 57(1): p. 74-81.

83. Wong, S.T. and M.S. Roos, A strategy for sampling on a sphere applied to 3D selective RF pulse design. Magn Reson Med, 1994. 32(6): p. 778-84.

84. Peters, D.C., et al., Undersampled projection reconstruction applied to MR angiography. Magn Reson Med, 2000. 43(1): p. 91-101.

85. Link, D.P., et al., New videodensitometric method for measuring renal artery blood flow at routine arteriography: validation in the canine model. Invest Radiol, 1979. 14(6): p. 465-70.

86. Marcus, M.L., C.J. Bischof, and D.D. Heistad, Comparison of microsphere and Xenon-133 clearance method in measuring skeletal muscle and cerebral blood flow. Circ Res, 1981. 48(5): p. 748-61.

87. Axel, L., Cerebral blood flow determination by rapid-sequence computed tomography: theoretical analysis. Radiology, 1980. 137(3): p. 679-86.

88. Taylor, K.J. and S. Holland, Doppler US. Part I. Basic principles, instrumentation, and pitfalls. Radiology, 1990. 174(2): p. 297-307.

89. Hoskins, P.R., Accuracy of maximum velocity estimates made using Doppler ultrasound systems. Br J Radiol, 1996. 69(818): p. 172-7.

90. Pelc, N.J., et al., Quantitative magnetic resonance flow imaging. Magn Reson Q, 1994. 10(3): p. 125-47. 
91. Dumoulin, C.L., Phase contrast MR angiography techniques. Magn Reson Imaging Clin N Am, 1995. 3(3): p. 399-411.

92. Pelc, N.J., et al., Phase contrast cine magnetic resonance imaging. Magn Reson Q, 1991. 7(4): p. 229-54.

93. Tan, R.S. and R.H. Mohiaddin, Cardiovascular applications of magnetic resonance flow measurement. Rays, 2001. 26(1): p. 71-91.

94. Lotz, J., et al., Cardiovascular flow measurement with phase-contrast MR imaging: basic facts and implementation. Radiographics, 2002. 22(3): p. 651-71.

95. Holland, G.A., et al., Breath-hold ultrafast three-dimensional gadolinium-enhanced MR angiography of the aorta and the renal and other visceral abdominal arteries. AJR Am J Roentgenol, 1996. 166(4): p. 971-81.

96. Prince, M.R., et al., Dynamic gadolinium-enhanced three-dimensional abdominal MR arteriography. J Magn Reson Imaging, 1993. 3(6): p. 877-81.

97. Snidow, J.J., et al., Three-dimensional gadolinium-enhanced MR angiography for aortoiliac inflow assessment plus renal artery screening in a single breath hold. Radiology, 1996. 198(3): p. 725-32.

98. Prince, M.R., Gadolinium-enhanced MR aortography. Radiology, 1994. 191(1): p. 155-64.

99. Jung, H.W., et al., Contrast-enhanced $M R$ angiography for the diagnosis of intracranial vascular disease: optimal dose of gadopentetate dimeglumine. AJR Am J Roentgenol, 1995. 165(5): p. 1251-5.

100. Katoh, M., et al., Free-breathing renal MR angiography with steady-state free-precession (SSFP) and slab-selective spin inversion: initial results. Kidney Int, 2004. 66(3): p. 1272-8.

101. Miyazaki, M. and H. Isoda, Non-contrast-enhanced MR angiography of the abdomen. Eur J Radiol, 2011. 80(1): p. 9-23.

102. Miyazaki, M. and M. Akahane, Non-contrast enhanced MR angiography: established techniques. J Magn Reson Imaging, 2012. 35(1): p. 1-19.

103. Dumoulin, C.L., et al., Two- and three-dimensional phase contrast MR angiography of the abdomen. J Comput Assist Tomogr, 1990. 14(5): p. 779-84.

104. Laub, G.A., Time-of-flight method of MR angiography. Magn Reson Imaging Clin N Am, 1995. 3(3): p. 391-8.

105. Masaryk, T.J., et al., Carotid-CNS MR flow imaging. Magn Reson Med, 1990. 14(2): p. 308-14.

106. Nishimura, D.G., Time-of-flight MR angiography. Magn Reson Med, 1990. 14(2): p. 194201.

107. Dumoulin, C.L., et al., Three-dimensional phase contrast angiography. Magn Reson Med, 1989. 9(1): p. 139-49.

108. Bolger, A.F., et al., Transit of blood flow through the human left ventricle mapped by cardiovascular magnetic resonance. J Cardiovasc Magn Reson, 2007. 9(5): p. 741-7.

109. Kilner, P.J., et al., Asymmetric redirection of flow through the heart. Nature, 2000. 404(6779): p. 759-61.

110. Kvitting, J.P., et al., Flow patterns in the aortic root and the aorta studied with timeresolved, 3-dimensional, phase-contrast magnetic resonance imaging: implications for aortic valve-sparing surgery. J Thorac Cardiovasc Surg, 2004. 127(6): p. 1602-7.

111. Markl, M., et al., Time-resolved 3-dimensional velocity mapping in the thoracic aorta: visualization of 3-directional blood flow patterns in healthy volunteers and patients. J Comput Assist Tomogr, 2004. 28(4): p. 459-68.

112. Vanninen, R., et al., Hemodynamic effects of carotid endarterectomy by magnetic resonance flow quantification. Stroke, 1995. 26(1): p. 84-9. 
113. Vanninen, R.L., et al., Carotid artery stenosis: clinical efficacy of MR phase-contrast flow quantification as an adjunct to MR angiography. Radiology, 1995. 194(2): p. 459-67.

114. Carvalho, J.L., J.F. Nielsen, and K.S. Nayak, Feasibility of in vivo measurement of carotid wall shear rate using spiral Fourier velocity encoded MRI. Magn Reson Med, 2010. 63(6): p. 1537-47.

115. Gatenby, J.C., T.R. McCauley, and J.C. Gore, Mechanisms of signal loss in magnetic resonance imaging of stenoses. Med Phys, 1993. 20(4): p. 1049-57.

116. Wakhloo, A.K., et al., Hemodynamics of carotid artery atherosclerotic occlusive disease. J Vasc Interv Radiol, 2004. 15(1 Pt 2): p. S111-21.

117. Urchuk, S.N. and D.B. Plewes, Mechanisms of flow-induced signal loss in $M R$ angiography. J Magn Reson Imaging, 1992. 2(4): p. 453-62.

118. Siegel, J.M., Jr., et al., Computational simulation of turbulent signal loss in $2 D$ time-offlight magnetic resonance angiograms. Magn Reson Med, 1997. 37(4): p. 609-14.

119. Ahn, C.B., et al., The effects of random directional distributed flow in nuclear magnetic resonance imaging. Med Phys, 1987. 14(1): p. 43-8.

120. Tang, C., D.D. Blatter, and D.L. Parker, Accuracy of phase-contrast flow measurements in the presence of partial-volume effects. J Magn Reson Imaging, 1993. 3(2): p. 377-85.

121. Firmin, D.N., et al., The application of phase shifts in NMR for flow measurement. Magn Reson Med, 1990. 14(2): p. 230-41.

122. Nayler, G.L., Jets associated with stenotic valvular disease. J Comput Assist Tomogr 1986. 10: p. 715-722.

123. Kilner, P.J., et al., Valve and great vessel stenosis: assessment with $M R$ jet velocity mapping. Radiology, 1991. 178(1): p. 229-35.

124. Kilner, P.J., et al., Magnetic resonance jet velocity mapping in mitral and aortic valve stenosis. Circulation, 1993. 87(4): p. 1239-48.

125. Spielmann, R.P., et al., Appearance of poststenotic jets in MRI: dependence on flow velocity and on imaging parameters. Magn Reson Imaging, 1991. 9(1): p. 67-72.

126. Tkach, J.A., et al., Three-dimensional time-of-flight MR angiography with a specialized gradient head coil. J Magn Reson Imaging, 1993. 3(2): p. 365-75.

127. Takahashi, M., et al., Ultra-short echo time (UTE) MR imaging of the lung: comparison between normal and emphysematous lungs in mutant mice. J Magn Reson Imaging, 2010. 32(2): p. 326-33.

128. Togao, O., et al., Ultrashort echo time (UTE) MRI of the lung: assessment of tissue density in the lung parenchyma. Magn Reson Med, 2010. 64(5): p. 1491-8.

129. Bae, W.C., et al., Ultrashort echo time MR imaging of osteochondral junction of the knee at $3 \mathrm{~T}$ : identification of anatomic structures contributing to signal intensity. Radiology, 2010. 254(3): p. 837-45.

130. Catana, C., et al., Toward implementing an MRI-based PET attenuation-correction method for neurologic studies on the MR-PET brain prototype. J Nucl Med, 2010. 51(9): p. 1431-8.

131. Keereman, V., et al., MRI-based attenuation correction for PET/MRI using ultrashort echo time sequences. J Nucl Med, 2010. 51(5): p. 812-8.

132. Benjamin, M., S. Milz, and G.M. Bydder, Magnetic resonance imaging of entheses. Part 2. Clin Radiol, 2008. 63(6): p. 704-11.

133. Benjamin, M., S. Milz, and G.M. Bydder, Magnetic resonance imaging of entheses. Part 1. Clin Radiol, 2008. 63(6): p. 691-703.

134. Chan, C.F., et al., Ultra-short echo time cardiovascular magnetic resonance of atherosclerotic carotid plaque. J Cardiovasc Magn Reson, 2010. 12: p. 17. 
135. Du, J., et al., Direct imaging and quantification of carotid plaque calcification. Magn Reson Med, 2011. 65(4): p. 1013-20.

136. Sharma, S., et al., Quantification of calcifications in endarterectomy samples by means of high-resolution ultra-short echo time imaging. Invest Radiol, 2010. 45(3): p. 109-13.

137. Gatehouse, P.D., et al., Contrast-enhanced MRI of the menisci of the knee using ultrashort echo time (UTE) pulse sequences: imaging of the red and white zones. $\mathrm{Br} \mathrm{J}$ Radiol, 2004. 77(920): p. 641-7.

138. Bydder, M., et al., The magic angle effect: a source of artifact, determinant of image contrast, and technique for imaging. J Magn Reson Imaging, 2007. 25(2): p. 290-300.

139. Bydder, G.M. and C.B. Chung, Magnetic resonance imaging of short T2 relaxation components in the musculoskeletal system. Skeletal Radiol, 2009. 38(3): p. 201-5.

140. Gold, G.E., et al., MR imaging of articular cartilage of the knee: new methods using ultrashort TEs. AJR Am J Roentgenol, 1998. 170(5): p. 1223-6.

141. Omoumi, P., et al., Imaging of lower limb cartilage. Top Magn Reson Imaging, 2009. 20(3): p. 189-201.

142. Koff, M.F. and H.G. Potter, Noncontrast MR techniques and imaging of cartilage. Radiol Clin North Am, 2009. 47(3): p. 495-504.

143. Williams, A., et al., Assessing degeneration of human articular cartilage with ultra-short echo time (UTE) T2* mapping. Osteoarthritis Cartilage, 2010. 18(4): p. 539-46.

144. Du, J., et al., Dual inversion recovery, ultrashort echo time (DIR UTE) imaging: creating high contrast for short-T(2) species. Magn Reson Med, 2010. 63(2): p. 447-55.

145. Hall-Craggs, M.A., et al., Ultrashort echo time (UTE) MRI of the spine in thalassaemia. $\mathrm{Br}$ J Radiol, 2004. 77(914): p. 104-10.

146. Carl, M., et al., Optimizing MR signal contrast of the temporomandibular joint disk. J Magn Reson Imaging, 2011. 34(6): p. 1458-64.

147. Chappell, K.E., et al., Magnetic resonance imaging of the liver with ultrashort TE (UTE) pulse sequences. J Magn Reson Imaging, 2003. 18(6): p. 709-13.

148. Hankins, J.S., et al., R2* magnetic resonance imaging of the liver in patients with iron overload. Blood, 2009. 113(20): p. 4853-5.

149. Waldman, A., et al., MRI of the brain with ultra-short echo-time pulse sequences. Neuroradiology, 2003. 45(12): p. 887-92.

150. Portman, O., et al., Magnetic resonance imaging of the normal pituitary gland using ultrashort TE (UTE) pulse sequences (REV 1.0). Neuroradiology, 2008. 50(3): p. 213-20.

151. Ahn, C.B. and Z.H. Cho, A new phase correction method in NMR imaging based on autocorrelation and histogram analysis. IEEE Trans Med Imaging, 1987. 6(1): p. 32-6.

152. Duijndam, A., Nonuniform Fast Fourier Transform. Geophysics, 1999. 64(2): p. 539-551.

153. Fredriksson-Ahomaa, M., et al., Yersiniosis in zoo marmosets (Callitrix jacchuss) caused by Yersinia enterocolitica 4/O:3. Vet Microbiol, 2007. 121(3-4): p. 363-7.

154. Harloff, A., et al., 3D blood flow characteristics in the carotid artery bifurcation assessed by flow-sensitive 4D MRI at 3T. Magn Reson Med, 2009. 61(1): p. 65-74.

155. Hope, M.D., et al., Bicuspid aortic valve: four-dimensional MR evaluation of ascending aortic systolic flow patterns. Radiology, 2010. 255(1): p. 53-61.

156. Kecskemeti, S., et al., High resolution three-dimensional cine phase contrast MRI of small intracranial aneurysms using a stack of stars k-space trajectory. J Magn Reson Imaging, 2012. 35(3): p. 518-27.

157. Stankovic, Z., et al., MR-based visualization and quantification of three-dimensional flow characteristics in the portal venous system. J Magn Reson Imaging, 2010. 32(2): p. 46675 . 
158. Uribe, S., et al., Four-dimensional (4D) flow of the whole heart and great vessels using real-time respiratory self-gating. Magn Reson Med, 2009. 62(4): p. 984-92.

159. Wigstrom, L., L. Sjoqvist, and B. Wranne, Temporally resolved 3D phase-contrast imaging. Magn Reson Med, 1996. 36(5): p. 800-3.

160. Pike, G.B., et al., Magnetic resonance velocity imaging using a fast spiral phase contrast sequence. Magn Reson Med, 1994. 32(4): p. 476-83.

161. Takahashi, A., et al., A Pulse Sequence for Flow Evaluation Based on Self-Refocused RF and Interleaved Spiral Readout. J Magn Reson, 1997. 126(1): p. 127-32.

162. Nishimura, D.G., P. Irarrazabal, and C.H. Meyer, A velocity k-space analysis of flow effects in echo-planar and spiral imaging. Magn Reson Med, 1995. 33(4): p. 549-56.

163. Kadbi, M., et al., A novel phase-corrected 3D cine ultra-short te (UTE) phase-contrast MRI technique. Conf Proc IEEE Eng Med Biol Soc, 2012. 2012: p. 77-81.

164. Sigfridsson, A., et al., Four-dimensional flow MRI using spiral acquisition. Magn Reson Med, 2012. 68(4): p. 1065-73.

165. Pelc, N.J., et al., Encoding strategies for three-direction phase-contrast MR imaging of flow. J Magn Reson Imaging, 1991. 1(4): p. 405-13.

166. Caruthers, S.D., et al., Practical value of cardiac magnetic resonance imaging for clinical quantification of aortic valve stenosis: comparison with echocardiography. Circulation, 2003. 108(18): p. 2236-43.

167. Carvalho, J.L. and K.S. Nayak, Rapid quantitation of cardiovascular flow using sliceselective fourier velocity encoding with spiral readouts. Magn Reson Med, 2007. 57(4): p. 639-46.

168. Sondergaard, L., et al., Mitral and aortic valvular flow: quantification with MR phase mapping. J Magn Reson Imaging, 1992. 2(3): p. 295-302.

169. Kadbi, M., et al., Validation of 3D ultra-short TE (UTE) Phase-Contrast MRI for imaging of steady flow: initial phantom experiments. Conf Proc IEEE Eng Med Biol Soc, 2012. 2012: p. 372-6.

170. Wetzel, S., et al., In vivo assessment and visualization of intracranial arterial hemodynamics with flow-sensitized 4D MR imaging at 3T. AJNR Am J Neuroradiol, 2007. 28(3): p. 433-8.

171. Frydrychowicz, A., et al., Visualization of iliac and proximal femoral artery hemodynamics using time-resolved 3D phase contrast MRI at 3T. J Magn Reson Imaging, 2007. 25(5): p. 1085-92.

172. Francois, C.J., et al., 4D cardiovascular magnetic resonance velocity mapping of alterations of right heart flow patterns and main pulmonary artery hemodynamics in tetralogy of Fallot. J Cardiovasc Magn Reson, 2012. 14: p. 16.

173. Bley, T.A., et al., Noninvasive assessment of transstenotic pressure gradients in porcine renal artery stenoses by using vastly undersampled phase-contrast MR angiography. Radiology, 2011. 261(1): p. 266-73.

174. Johnson, K.M., et al., Improved 3D phase contrast MRI with off-resonance corrected dual echo VIPR. Magn Reson Med, 2008. 60(6): p. 1329-36.

175. Dumoulin, C.L., et al., Simultaneous acquisition of phase-contrast angiograms and stationary-tissue images with Hadamard encoding of flow-induced phase shifts. J Magn Reson Imaging, 1991. 1(4): p. 399-404.

176. Callahan, M.J., et al., Validation of instantaneous pressure gradients measured by continuous-wave Doppler in experimentally induced aortic stenosis. Am J Cardiol, 1985. 56(15): p. 989-93. 
177. American College of Cardiology/American Heart Association Task Force on Practice, G., et al., ACC/AHA 2006 guidelines for the management of patients with valvular heart disease: a report of the American College of Cardiology/American Heart Association Task Force on Practice Guidelines (writing committee to revise the 1998 Guidelines for the Management of Patients With Valvular Heart Disease): developed in collaboration with the Society of Cardiovascular Anesthesiologists: endorsed by the Society for Cardiovascular Angiography and Interventions and the Society of Thoracic Surgeons. Circulation, 2006. 114(5): p. e84-231.

178. Cawley, P.J., J.H. Maki, and C.M. Otto, Cardiovascular magnetic resonance imaging for valvular heart disease: technique and validation. Circulation, 2009. 119(3): p. 468-78.

179. Waters, E.A., S.D. Caruthers, and S.A. Wickline, Correlation analysis of stenotic aortic valve flow patterns using phase contrast MRI. Ann Biomed Eng, 2005. 33(7): p. 878-87.

180. Dulce, M.C., et al., Severity of aortic regurgitation: interstudy reproducibility of measurements with velocity-encoded cine MR imaging. Radiology, 1992. 185(1): p. 23540.

181. Fujita, N., et al., Quantification of mitral regurgitation by velocity-encoded cine nuclear magnetic resonance imaging. J Am Coll Cardiol, 1994. 23(4): p. 951-8.

182. Sondergaard, L., et al., Quantification of aortic regurgitation by magnetic resonance velocity mapping. Am Heart J, 1993. 125(4): p. 1081-90.

183. O'Brien, K.R., et al., Aortic valve stenotic area calculation from phase contrast cardiovascular magnetic resonance: the importance of short echo time. J Cardiovasc Magn Reson, 2009. 11: p. 49.

184. Yap, S.C., et al., A simplified continuity equation approach to the quantification of stenotic bicuspid aortic valves using velocity-encoded cardiovascular magnetic resonance. J Cardiovasc Magn Reson, 2007. 9(6): p. 899-906.

185. Eichenberger, A.C., R. Jenni, and G.K. von Schulthess, Aortic valve pressure gradients in patients with aortic valve stenosis: quantification with velocity-encoded cine $M R$ imaging. AJR Am J Roentgenol, 1993. 160(5): p. 971-7.

186. Garcia, J., Kadem, L., Larose, E., Clavel, M.A., Pibarot, P., Comparison between cardiovascular magnetic resonance and transthoracic doppler echocardiography for the estimation of effective orifice area in aortic stenosis. Journal of Cardiovascular Magnetic Resonance, 2011. 13(25): p. 9.

187. Negahdar, M.J.K., M.; Kotys, M.; Alshaher, M.; Fischer, S.; Amini, A. A., Rapid flow quantification in iliac arteries with spiral phase-contrast MRI. EMBC Annual Int. Conf. of the IEEE, 2011: p. 2804-2808.

188. Pruessmann, K.P., et al., SENSE: sensitivity encoding for fast MRI. Magn Reson Med, 1999. 42(5): p. 952-62.

189. Griswold, M.A., et al., Generalized autocalibrating partially parallel acquisitions (GRAPPA). Magn Reson Med, 2002. 47(6): p. 1202-10.

190. Tsao, J., P. Boesiger, and K.P. Pruessmann, $k$-t BLAST and k-t SENSE: dynamic MRI with high frame rate exploiting spatiotemporal correlations. Magn Reson Med, 2003. 50(5): p. 1031-42.

191. Lustig, M., D. Donoho, and J.M. Pauly, Sparse MRI: The application of compressed sensing for rapid MR imaging. Magn Reson Med, 2007. 58(6): p. 1182-95. 


\section{CURRICULUM VITAE}

CONTACT INFORMATION

Address: $\quad$ Lutz Hall, Room 308

Department of Electrical and Computer Engineering, University of Louisville Louisville, KY 40292

Phone: (502) 8523400

Email: $\quad$ m0kadb01@louisville.edu

\section{EDUCATION}

Ph.D. Candidate, Electrical Engineering $(G P A=3.93 / 4.00)$

University of Louisville, Louisville, KY

Thesis title: Ultra-Short TE (UTE) Phase-Contrast (PC) MRI for

Assessment of Stenotic Flow and Hemodynamics

M.S., Biomedical Engineering (GPA= 3.5/4.00)

$2004-2006$

Sharif University of Technology, Tehran, Iran

Thesis title: Coloring of MR images of human brain using segmentation methods

\section{B.S., Biomedical Engineering}

Shahed University, Tehran, Iran

2000- 2004

Thesis title: Design and implementation of fetal heart detector (Sonicaid)

\section{RESEARCH INTERESTS}

- Cardiac and Cardiovascular MRI imaging, reconstruction and analysis

- Flow imaging and quantification methods

- MRI clinical applications and sequence programming and optimization

PROFESSIONAL EXPERIENCE 
- Medical Imaging Lab, Dept. of Electrical and Computer Engineering

Doctoral student in Electrical and Computer Engineering

Sept 2008-Dec 2013

- Took courses in Image Processing (A), Medical imaging systems (A), Medical imaging analysis(A+), Digital signal processing (A+),

Combinatorial optimization (A), Communication and Modulation (A), Random process and estimation (A+), Foundation of optimization (A).

- Research assistant in Cardiovascular MRI and Image Processing

- Philips healthcare, Clinical scientists group, Cleveland, $\mathbf{O H}$

Internship and joint research program

April 2011- Dec 2013

- Cardiac and cardiovascular MR Imaging and sequence development, reconstruction, and image analysis as part of research agreement and collaboration with the University of Louisville

- Teaching Assistant: Sequence development mode (SDM), pulse programming environment (PPE), Coil workshop

- Ganjineh Rah Toos, Tehran, Iran

Project Manager

June 2006-June 2008

- Design and consult hospitals and medical imaging centers construction

- Consultant on equipping hospitals and medical imaging centers

- Vesta Biomedical Engineering Co., Tehran, Iran

Technical and Training Engineer

May 2004-July 2007

- Product specialist in biomedical equipment and device

- Technical and training specialist in sleep diagnostics and treatment systems, EEG, ECG, Quality assurance, and medical equipment calibration systems

\section{PUBLICATIONS}

\section{Book Chapter and Peer-reviewed Full-length Articles:}

1. Flow Imaging with MRI, MJ Negahdar, Mo Kadbi, and Amir Amini, in Physics of cardiovascular and neurovascular imaging, Carlo Cavedon and Stephen Rudin (Eds.), Taylor and Francis, forthcoming, 2013 
2. 4D UTE Flow: A Phase-Contrast MRI Technique for Assessment and Visualization of Stenotic Flows. Mo Kadbi, MJ Negahdar, Jung won Cha, Melanie Traughber, Peter Martin, Marcus Stoddard, and Amir A. Amini, Magnetic Resonance in Medicine, under revision , 2013

3. Assessment of Flow and Hemodynamics in the Carotid Artery Using a Reduced TE 4D Flow Spiral Phase-Contrast MRI, Mo Kadbi, Mj Negahdar, Melanie Traughber, Peter Martin, and Amir A Amini. IEEE Engineering in Medicine and Biology Conference, Japan, 2013

4. Noninvasive 3D Pressure Calculation From PC-MRI Via Non-Iterative Harmonics-based Orthoghonal Projection: Constant Flow Experiment, MJ Negahdar, Jung won Cha, Mo Kadbi, Juan Cebral, Amir Amini, IEEE Engineering in Medicine and Biology Conference, Japan, 2013

5. Comparison of cartesian, UTE radial, and spiral Phase-Contrast MRI in measurement of blood flow in extracranial carotid arteries: normal subjects., MJ Negahdar, Mo Kadbi, Vahid Tavakoli, Jens Heidenreich, Andrea Yancey, Amir A. Amini, SPIE Medical Imaging Symposium, Orlando, Florida, February 2013.

6. A Novel Phase-Corrected 3D Cine Ultra-Short TE (UTE) Phase-Contrast MRI Technique., Mo Kadbi, Hui Wang, MJ Negahdar, Lizette Warner, Melanie Traughber, Peter Martin, Jens Heidenreich, and Amir A Amini, Conf Proc IEEE Eng Med Biol Soc. 2012 Aug;2012:77-81. PMID: 23365836.

7. Validation of 3D Ultra-Short TE (UTE) Phase-Contrast MRI For Imaging of Steady Flow: Initial Phantom Experiments., Mo Kadbi, M Negahdar , JW Cha, M Traughber, P Martin, AA Amini, Conf Proc IEEE Eng Med Biol Soc. 2012 Aug;2012:372-6. PMID: 23365907

8. Cardiac Deformation Analysis from Orthogonal CSPAMM (OCSPAMM) Tagged MRI., Hui Wang, Mo Kadbi, Motaz Alshaher, Melanie Kotys, Stefan E. Fischer, and Amir A. Amini,, Society for Cardiovascular Magnetic Resonance (SCMR), Orlando, FL, February 2012, Journal of Cardiovascular Magnetic Resonance 2012, 14(suppl. 1):W17

9. Orthogonal CSPAMM (OCSPAMM) MR Tagging for Imaging Ventricular Wall Motion., H. Wang, Mo Kadbi, M. S. Kotys, M. Ersoy, G. P. Chatzimavroudis, R. M. Setser, S. E. Fischer, and A. A. Amini. Conf Proc IEEE Eng Med Biol Soc. 2011;2011:535-8. PMID: 22254366.

10. Rapid flow quantification in iliac arteries with spiral phase-contrast MRI., M. Negahdar, Mo Kadbi, M. Kotys, S. Fischer, and A. A. Amini., Proc of IEEE Eng Med Biol Soc. 2011;2011:2804-8. PMID: 22254924.

11. An Improved Real-time Cine Late Gadolinium Enhancement (LGE) Imaging Method at 3T., Mo Kadbi, Melanie Kotys, Motaz Alshaher, Stefan Fischer, and Amir A. Amini. Proc of IEEE Eng Med Biol Soc. 2011;2011:531-4. PMID: 22254365. 
12. A new method for colorizing of Multichannel MR Images based on real color of human brain., Mo Kadbi , E. Fatemizadeh, and A. Eslami; EUSIPCO Conference, Poland, 2007

13. Classification of ECG Arrhythmias Based on Statistical and Time-Frequency Features., Mo.Kadbi, J. Hashemi, Medical Signal \& Information Processing (MEDSIP), July 2006 Glasgow, UK.

14. Automatic detection of epileptic seizure using Time-Frequency Distribution., H.R.Mohseni, A.Maghsoudi, Mo Kadbi, Medical Signal \& Information Processing(MEDSIP), July 2006, Glasgow, UK.

15. Epileptic Seizure Detection Using Neural Fuzzy Networks., H.R.Mohseni, A.Maghsoudi, Mo Kadbi, FUZZ-IEEE conference, Canada, 2006.

\section{International Conference Abstracts:}

1- 4D UTE Flow: A Novel 4D Ultra-Short TE Phase-Contrast MRI Technique for Assessment of Flow and Hemodynamics., Mo Kadbi, Melanie Traughber, Peter Martin, and Amir A Amini, Submitted to $21^{\text {st }}$ International Society of Magnetic Resonance in Medicine (ISMRM) Annual Meeting, Salt lake city, Utah, April 2013.

2- A Fast Reduced TE 4D Spiral PC MRI Sequence for Assessment of Flow and Hemodynamics., Mo Kadbi, Mj Negahdar, Melanie Traughber, Peter Martin, and Amir A Amini, Submitted to $21^{\text {st }}$ International Society of Magnetic Resonance in Medicine (ISMRM) Annual Meeting, Salt lake city, Utah, April 2013.

3- A Fast 3D non-Iterative Approach to Pressure Calculation from PC-MRI: Phantom Experiments., MJ Negahdar, Mo Kadbi, JungWon Cha, Juan Cebral, and Amir Amini, Submitted to $21^{\text {st }}$ International Society of Magnetic Resonance in Medicine (ISMRM) Annual Meeting, Salt lake city, Utah, April 2013.

4- 3D Cine Ultra-short TE (UTE) phase contrast imaging in carotid artery: comparison with conventional technique., Mo Kadbi, Hui Wang, Lizette Warner, Melanie Traughber, Motaz Alshaher, Andrea Yancey, Jens Heidenreich, and Amir A Amini, International Society of Magnetic Resonance in Medicine Annual Meeting, Melbourne, Australia, May 2012.

5- Effect of Trajectory Delay in Cine Ultra-Short TE Phase-Contrast MR Imaging of the Carotid Bifurcation., Mo Kadbi, Hui Wang, Lizette Warner, Melanie Traughber, Motaz Alshaher, Andrea Yancey, Jens Heidenreich, and Amir A Amini, International Society of Magnetic Resonance in Medicine Annual Meeting, Melbourne, Australia, May 2012. 
6- Choice of Bandpass Filter on Accuracy of Myocardial Deformation Recovery With SinMod., Hui Wang, Mo Kadbi, Melanie Troughber, and Amir Amini, International Society of Magnetic Resonance in Medicine Annual Meeting, Melbourne, Australia, May 2012.

7- A real-time cine late gadolinium enhancement imaging method at 3T., Mo

Kadbi, Hui Wang, MJ Negahdar, Melanie Kotys, Stefan Fischer, and Amir A. Amini, ISMRM 19th annual meeting, Montreal, Canada, May 2011.

\section{PRESENTATIOS}

1. 4D Flow MR Imaging using Ultra-short TE (UTE) technique., Mo Kadbi, Cincinnati Children's Hospital Medical Center, 2013

2. A new method for colorizing of Multichannel MR Images based on real color of human brain., Mo Kadbi , E. Fatemizadeh, and A. Eslami; EUSIPCO Conference, Poland, 2007

3. An Improved Real-time Cine Late Gadolinium Enhancement (LGE) Imaging Method at 3T., Mo Kadbi, Melanie Kotys, Motaz Alshaher, Stefan Fischer, and Amir A. Amini. Graduate Research Symposium, March 2011, University of Louisville, Louisville, KY, USA.

4. 3D Cine Ultra-short TE (UTE) phase contrast imaging in carotid artery: comparison with conventional technique., Mo Kadbi, Hui Wang, Lizette Warner, Melanie Traughber, Motaz Alshaher, Andrea Yancey, Jens Heidenreich, and Amir A Amini, Research!Louisville, Oct 2012, University of Louisville, Louisville, KY, USA.

5. A Novel Phase-Corrected 3D Cine Ultra-Short TE (UTE) Phase-Contrast MRI Technique., Mo Kadbi, Hui Wang, MJ Negahdar, Lizette Warner, Melanie Traughber, Peter Martin, Jens Heidenreich, and Amir A Amini, IEEE Engineering in Medicine and Biology Conference, San Diego, CA, September 2012

\section{REVIEWER OF JOURNALS AMD CONFERENCES}

- IEEE Transactions on Medical Imaging

- $\quad$ MICCAI, International Conference on Medical Image Computing and Computer Assisted Intervention

- $\quad$ SPIE, Conference on Biomedical Applications in Molecular, Structural, and Functional Imaging

\section{PROFESSIONAL SOCIETIES}


- Member, IEEE Engineering in Medicine and Biology Society (EMBS)

- Member, Institute of Electrical and Electronics Engineers (IEEE)

- Member, International Society of Magnetic Resonance in Medicine (ISMRM)

\section{AWARDS AND HONORS}

- University of Louisville Fellows and Graduate Award.

$2008-2010$

- Ranked 6th among all 20000 students participating in the university entrance exam for M.S. in biomedical engineering.

- Invited member of young researchers association.

- Ranked 190th in Iran national exam of university entrance among more than 500,000 applicants (Top 0.09\%).

\section{PROGRAMMING SKILLS}

- Proficient in Matlab, $\mathrm{C}++$, Philips GOALC pulse programming language, AutoCAD.

- Statistical signal and image processing 